\title{
Silver-Catalyzed, Chemo- and Enantioselective Intramolecular Dearomatization of Indoles to Access Sterically Congested Azaspiro Frameworks
}

Jun Ueda, ${ }^{\dagger}$ Shingo Harada, ${ }^{*},{ }^{\dagger}$ Ayaka Kanda ${ }^{\dagger}$ Hiroki Nakayama, ${ }^{\dagger}$ and Tetsuhiro Nemoto ${ }^{*}, \dagger$

†Graduate School of Pharmaceutical Sciences, Chiba University, 1-8-1, Inohana, Chuo-ku, Chiba 260-8675, Japan

Molecular Chirality Research Center, Chiba University, 1-33, Yayoi-cho, Inage-ku, Chiba 263-8522, Japan

E-mail : tnemoto@faculty.chiba-u.jp,Sharada@chiba-u.jp

\section{Table of Contents}

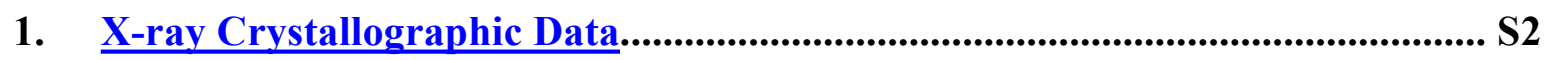

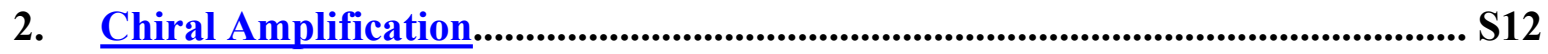

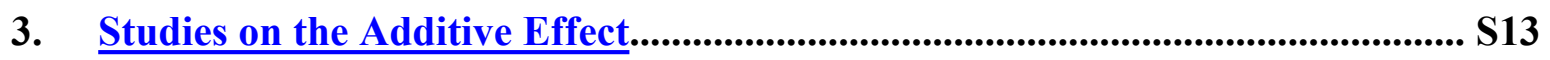

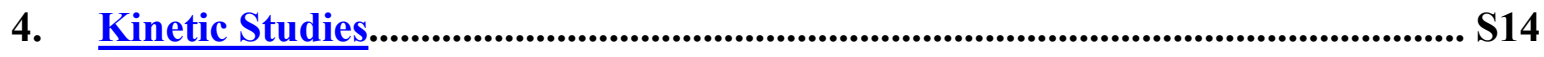

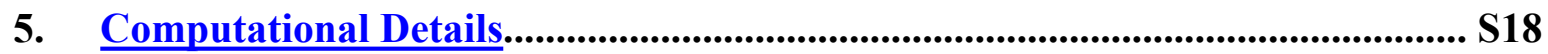

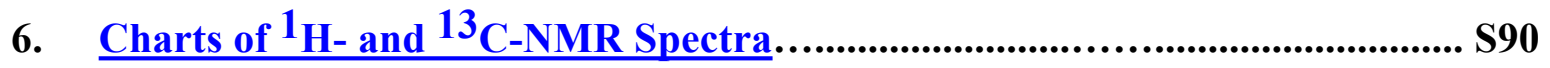

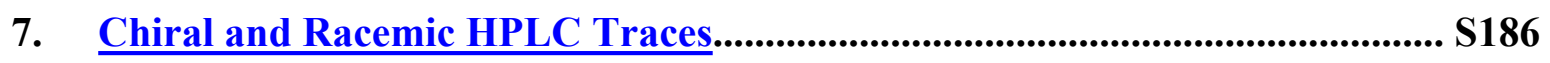

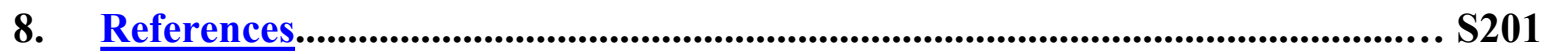




\section{X-ray Crystallographic Data}

ORTEP of 3b, CCDC No. 1959308

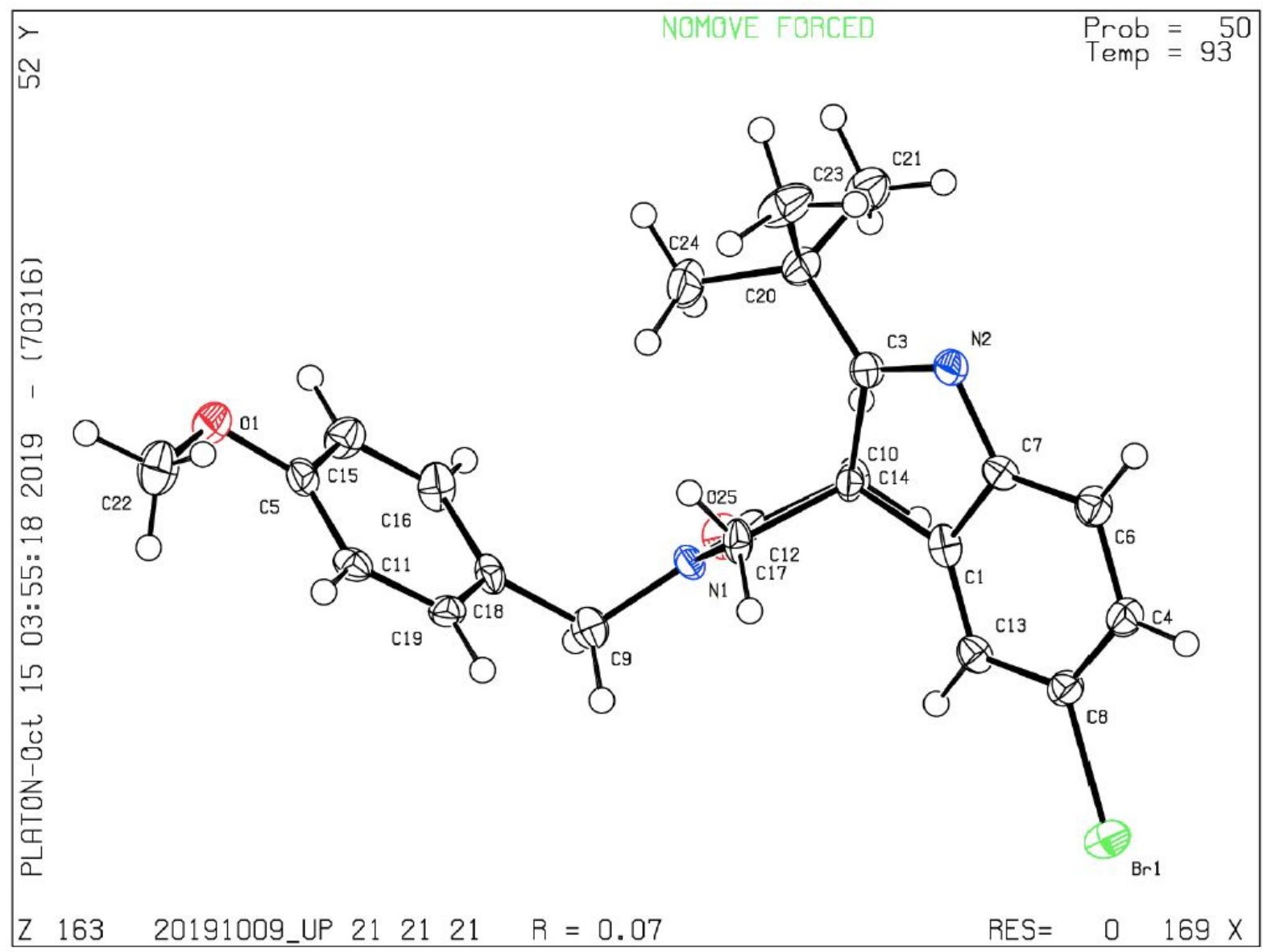

The ellipsoid contour probability level in the ORTEP is 50\%.

\section{Experimental}

Data Collection

A colorless block crystal of $\mathrm{C} 23 \mathrm{H} 25 \mathrm{BrN} 2 \mathrm{O} 2$ having approximate dimensions of $0.200 \mathrm{x}$ $0.200 \times 0.100 \mathrm{~mm}$ was mounted on a glass fiber. All measurements were made on a Rigaku R-AXIS RAPID diffractometer using filtered $\mathrm{Cu}-\mathrm{Ka}$ radiation.

The crystal-to-detector distance was $127.40 \mathrm{~mm}$.

Cell constants and an orientation matrix for data collection corresponded to a primitive orthorhombic cell with dimensions:

$$
\begin{aligned}
& \mathrm{a}=9.4438(2) \AA \\
& \mathrm{b}=12.2974(2) \AA \\
& \mathrm{c}=18.0320(4) \AA
\end{aligned}
$$




$$
\mathrm{V}=2094.13(7) \AA 3
$$

For $\mathrm{Z}=4$ and F.W. $=441.37$, the calculated density is $1.400 \mathrm{~g} / \mathrm{cm} 3$. The reflection conditions of:

h00: $\mathrm{h}=2 \mathrm{n}$

$0 \mathrm{k} 0: \mathrm{k}=2 \mathrm{n}$

001: $1=2 \mathrm{n}$

uniquely determine the space group to be:

P212121 (\#19)

The data were collected at a temperature of $-180+10 \mathrm{C}$ to a maximum $2 \mathrm{q}$ value of 136.4o. A total of 30 oscillation images were collected. A sweep of data was done using $\mathrm{w}$ scans from 80.0 to $260.0 \mathrm{o}$ in $30.00 \mathrm{o} \mathrm{step}$, at $\mathrm{c}=54.0 \mathrm{o}$ and $\mathrm{f}=0.0 \mathrm{o}$. The exposure rate was 4.0 [sec./o]. A second sweep was performed using w scans from 80.0 to $260.0 \mathrm{o}$ in $30.00 \mathrm{o}$ step, at $\mathrm{c}=54.0 \mathrm{o}$ and $\mathrm{f}=90.0 \mathrm{o}$. The exposure rate was 4.0 [sec./o]. Another sweep was performed using w scans from 80.0 to $260.0 \mathrm{o}$ in $30.00 \mathrm{o}$ step, at $\mathrm{c}=54.0 \mathrm{o}$ and $\mathrm{f}=180.0 \mathrm{o}$. The exposure rate was 4.0 [sec./o]. Another sweep was performed using w scans from 80.0 to 260.00 in $30.00 \mathrm{o}$ step, at $\mathrm{c}=54.0 \mathrm{o}$ and $\mathrm{f}=270.0 \mathrm{o}$. The exposure rate was 4.0 [sec./o]. Another sweep was performed using $\mathrm{w}$ scans from 80.0 to $260.0 \mathrm{o}$ in $30.00 \mathrm{o}$ step, at $\mathrm{c}=0.0 \mathrm{o}$ and $\mathrm{f}=0.0 \mathrm{o}$. The exposure rate was 4.0 [sec./o]. The crystal-to-detector distance was $127.40 \mathrm{~mm}$. Readout was performed in the $0.100 \mathrm{~mm}$ pixel mode.

\section{EXPERIMENTAL DETAILS}

The sample for X-ray crystal structure analysis was prepared by vapor diffusion method. A pre-dried $1 \mathrm{~mL}$ sample tube was charged with $\mathbf{3 b}$, which was dissolved in $\mathrm{CH}_{2} \mathrm{Cl}_{2}$. The sample tube was left to stand in a $5 \mathrm{~mL}$ sample tube charged with $n$-hexane until a single crystal forms.

\section{A. Crystal Data}

Empirical Formula

Formula Weight

Crystal Color, Habit

Crystal Dimensions

Crystal System

Lattice Type

\section{$\mathrm{C}_{23} \mathrm{H}_{25} \mathrm{BrN}_{2} \mathrm{O}_{2}$}

\subsection{7}

colorless, block

$0.200 \times 0.200 \times 0.100 \mathrm{~mm}$

orthorhombic

Primitive 
Lattice Parameters

$$
\begin{aligned}
& \mathrm{a}=9.4438(2) \AA \\
& \mathrm{b}=12.2974(2) \AA \\
& \mathrm{c}=18.0320(4) \AA \\
& \mathrm{V}=2094.13(7) \AA^{3}
\end{aligned}
$$

Space Group

$$
\mathrm{P} 2{ }_{1}{ }_{1}{ }^{2} 1 \text { (\#19) }
$$

$\mathrm{Z}$ value

4

$\mathrm{D}_{\text {calc }}$

$1.400 \mathrm{~g} / \mathrm{cm}^{3}$

$\mathrm{F}_{000}$

912.00

$\mathrm{m}(\mathrm{CuKa})$

$28.365 \mathrm{~cm}^{-1}$

B. Intensity Measurements

Diffractometer

Radiation

Voltage, Current

Temperature

Detector Aperture

Data Images

W oscillation Range $(c=54.0, f=0.0)$

Exposure Rate

W oscillation Range $(c=54.0, f=90.0)$

Exposure Rate

w oscillation Range $(c=54.0, f=180.0)$

Exposure Rate
R-AXIS RAPID

$\operatorname{CuKa}(\mathrm{I}=1.54187 \AA)$

$40 \mathrm{kV}, 30 \mathrm{~mA}$

$-180.0^{\circ} \mathrm{C}$

$460.0 \times 256.0 \mathrm{~mm}$

30 exposures

$80.0-260.0^{\circ}$

$4.0 \mathrm{sec} . / \mathrm{O}$

$80.0-260.0^{\circ}$

$4.0 \mathrm{sec} . / \mathrm{O}$

$80.0-260.0^{\circ}$

$4.0 \mathrm{sec} .{ }^{\circ}$ 
w oscillation Range $(c=54.0, f=270.0)$

Exposure Rate

w oscillation Range $(c=0.0, f=0.0)$

Exposure Rate

Detector Position

Pixel Size

$2 q_{\max }$

No. of Reflections Measured

Corrections $80.0-260.0^{\circ}$

$4.0 \mathrm{sec} . / \mathrm{O}$

$80.0-260.0^{\circ}$

$4.0 \mathrm{sec} . / \mathrm{O}$

$127.40 \mathrm{~mm}$

$0.100 \mathrm{~mm}$

$136.4^{\circ}$

Total: 21688

Unique: $3836\left(\mathrm{R}_{\text {int }}=0.1037\right)$

Friedel pairs: 1638

Lorentz-polarization

Absorption

(trans. factors: $0.554-0.753$ )

C. Structure Solution and Refinement

Structure Solution

Refinement

Function Minimized

Least Squares Weights

$2 q_{\max }$ cutoff

Anomalous Dispersion

No. Observations (All reflections)

No. Variables
Direct Methods

Full-matrix least-squares on $\mathrm{F}^{2}$

$\mathrm{Sw}\left(\mathrm{Fo}^{2}-\mathrm{Fc}^{2}\right)^{2}$

$\mathrm{w}=1 /\left[\mathrm{s}^{2}\left(\mathrm{Fo}^{2}\right)+(0.0494 \cdot \mathrm{P})^{2}\right.$

$+0.0000 \cdot \mathrm{P}$ ]

where $\mathrm{P}=\left(\operatorname{Max}\left(\mathrm{Fo}^{2}, 0\right)+2 \mathrm{Fc}^{2}\right) / 3$

$136.4^{0}$

All non-hydrogen atoms

3836

253 
Reflection/Parameter Ratio

Residuals: R1 (I>2.00s(I))

Residuals: R (All reflections)

Residuals: wR2 (All reflections)

Goodness of Fit Indicator

Flack Parameter $($ Friedel pairs $=1638)$

Max Shift/Error in Final Cycle

Maximum peak in Final Diff. Map

Minimum peak in Final Diff. Map
15.16

0.0674

0.0770

0.1305

1.066

$0.01(3)$

0.000

$0.53 \mathrm{e}^{-} / \AA^{3}$

$-0.77 \mathrm{e}^{-} / \AA^{3}$ 
ORTEP of 12, CCDC No. 2005341

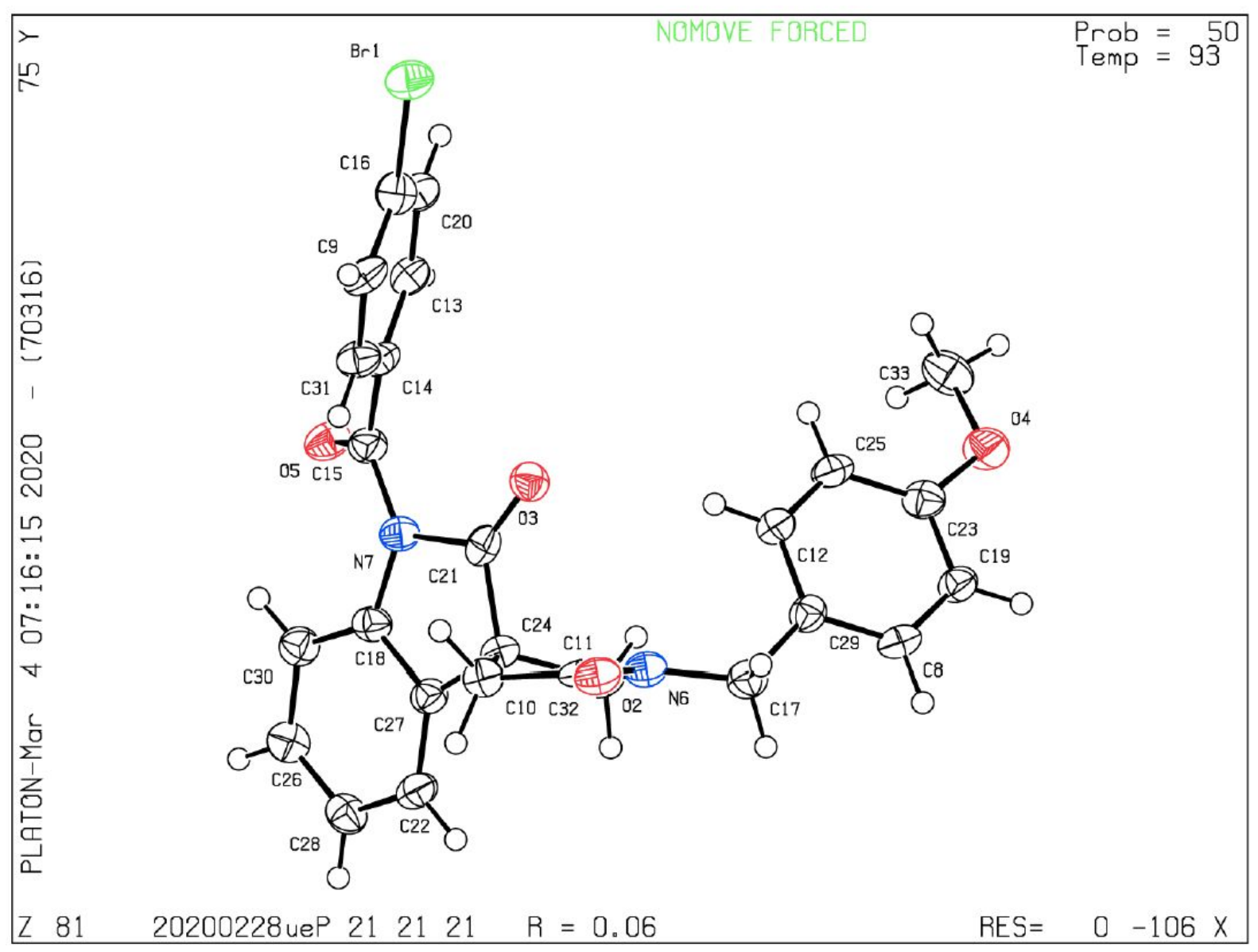

The ellipsoid contour probability level in the ORTEP is 50\%.

\section{Experimental}

\section{Data Collection}

A colorless block crystal of $\mathrm{C} 26 \mathrm{H} 21 \mathrm{BrN} 2 \mathrm{O} 4$ having approximate dimensions of $0.200 \mathrm{x}$ $0.100 \times 0.100 \mathrm{~mm}$ was mounted on a glass fiber. All measurements were made on a Rigaku $\mathrm{R}$-AXIS RAPID diffractometer using multi-layer mirror monochromated $\mathrm{Cu}-\mathrm{Ka}$ radiation.

The crystal-to-detector distance was $127.40 \mathrm{~mm}$.

Cell constants and an orientation matrix for data collection corresponded to a primitive orthorhombic cell with dimensions:

$$
\begin{gathered}
\mathrm{a}=6.3409(2) \AA \\
\mathrm{b}=15.7289(6) \AA \\
\mathrm{c}=21.5122(9) \AA \\
\mathrm{V}=2145.54(14) \AA 3
\end{gathered}
$$


For $\mathrm{Z}=4$ and F.W. $=505.37$, the calculated density is $1.564 \mathrm{~g} / \mathrm{cm} 3$. The reflection conditions of:

$\mathrm{h} 00: \mathrm{h}=2 \mathrm{n}$

$0 \mathrm{k} 0: \mathrm{k}=2 \mathrm{n}$

001: $1=2 \mathrm{n}$

uniquely determine the space group to be:

P212121 (\#19)

The data were collected at a temperature of $-180+1 \mathrm{oC}$ to a maximum $2 \mathrm{q}$ value of 136.4o. A total of 45 oscillation images were collected. A sweep of data was done using w scans from 80.0 to $260.0 \mathrm{o}$ in $20.00 \mathrm{o}$ step, at $\mathrm{c}=54.0 \mathrm{o}$ and $\mathrm{f}=0.0 \mathrm{o}$. The exposure rate was 7.0 [sec./o]. A second sweep was performed using w scans from 80.0 to $260.0 \mathrm{o}$ in $20.00 \mathrm{o}$ step, at $\mathrm{c}=54.0 \mathrm{o}$ and $\mathrm{f}=90.0 \mathrm{o}$. The exposure rate was 7.0 [sec./o]. Another sweep was performed using w scans from 80.0 to $260.0 \mathrm{o}$ in $20.00 \mathrm{o}$ step, at $\mathrm{c}=54.0 \mathrm{o}$ and $\mathrm{f}=180.0 \mathrm{o}$. The exposure rate was 7.0 [sec./o]. Another sweep was performed using w scans from 80.0 to $260.0 \mathrm{o}$ in $20.00 \mathrm{o}$ step, at $\mathrm{c}=54.0 \mathrm{o}$ and $\mathrm{f}=270.0 \mathrm{o}$. The exposure rate was 7.0 [sec./o]. Another sweep was performed using $\mathrm{w}$ scans from 80.0 to $260.0 \mathrm{o}$ in $20.00 \mathrm{o}$ step, at $\mathrm{c}=0.0 \mathrm{o}$ and $\mathrm{f}=0.0 \mathrm{o}$. The exposure rate was 7.0 [sec./o]. The crystal-to-detector distance was $127.40 \mathrm{~mm}$. Readout was performed in the $0.100 \mathrm{~mm}$ pixel mode.

\section{EXPERIMENTAL DETAILS}

The sample for X-ray crystal structure analysis was prepared by vapor diffusion method. A pre-dried $1 \mathrm{~mL}$ sample tube was charged with 12 , which was dissolved in $\mathrm{CH}_{2} \mathrm{Cl}_{2}$. The sample tube was left to stand in a $5 \mathrm{~mL}$ sample tube charged with $n$-hexane until a single crystal forms.

\section{A. Crystal Data}

Empirical Formula

Formula Weight

Crystal Color, Habit

Crystal Dimensions

Crystal System

Lattice Type

\section{$\mathrm{C}_{26} \mathrm{H}_{21} \mathrm{BrN}_{2} \mathrm{O}_{4}$}

505.37

colorless, block

$0.200 \times 0.100 \times 0.100 \mathrm{~mm}$

orthorhombic

Primitive 
Lattice Parameters

Space Group

$Z$ value

$\mathrm{D}_{\text {calc }}$

$\mathrm{F}_{000}$

$\mathrm{m}(\mathrm{CuKa})$

$$
\begin{aligned}
& \mathrm{a}=6.3409(2) \AA \\
& \mathrm{b}=15.7289(6) \AA \\
& \mathrm{c}=21.5122(9) \AA \\
& \mathrm{V}=2145.54(14) \AA^{3}
\end{aligned}
$$

$\mathrm{P} 2{ }_{1}{ }_{1}{ }_{1}(\# 19)$

4

$1.564 \mathrm{~g} / \mathrm{cm}^{3}$

1032.00

$29.323 \mathrm{~cm}^{-1}$

B. Intensity Measurements

Diffractometer

Radiation

$\mathrm{CuKa}(\mathrm{I}=1.54187 \AA)$

multi-layer mirror monochromated

Voltage, Current

$40 \mathrm{kV}, 30 \mathrm{~mA}$

Temperature

$-180.0^{\circ} \mathrm{C}$

Detector Aperture

$460.0 \times 256.0 \mathrm{~mm}$

Data Images

45 exposures

W oscillation Range $(\mathrm{c}=54.0, \mathrm{f}=0.0)$

$80.0-260.0^{\circ}$

Exposure Rate

$7.0 \mathrm{sec} . / \mathrm{O}$

W oscillation Range $(\mathrm{c}=54.0, \mathrm{f}=90.0)$

$80.0-260.0^{\circ}$

Exposure Rate

$7.0 \mathrm{sec} . / \mathrm{O}$

w oscillation Range $(\mathrm{c}=54.0, \mathrm{f}=180.0)$

$80.0-260.0^{\circ}$

Exposure Rate

$7.0 \mathrm{sec} . / \mathrm{O}$ 
w oscillation Range $(c=54.0, f=270.0)$

Exposure Rate

w oscillation Range $(c=0.0, f=0.0)$

Exposure Rate

Detector Position

Pixel Size

$2 q_{\max }$

No. of Reflections Measured

Corrections $80.0-260.0^{\circ}$

$7.0 \mathrm{sec} . / \mathrm{O}$

$80.0-260.0^{\circ}$

$7.0 \mathrm{sec} . / \mathrm{O}$

$127.40 \mathrm{~mm}$

$0.100 \mathrm{~mm}$

$136.4^{\circ}$

Total: 24056

Unique: $3927\left(\mathrm{R}_{\text {int }}=0.0994\right)$

Friedel pairs: 1651

Lorentz-polarization

Absorption

(trans. factors: $0.399-0.746$ )

C. Structure Solution and Refinement

Structure Solution

Refinement

Function Minimized

Least Squares Weights

$2 q_{\max }$ cutoff

Anomalous Dispersion

No. Observations (All reflections)

No. Variables
Direct Methods (SIR92)

Full-matrix least-squares on $\mathrm{F}^{2}$

$\mathrm{Sw}\left(\mathrm{Fo}^{2}-\mathrm{Fc}^{2}\right)^{2}$

$\mathrm{w}=1 /\left[\mathrm{s}^{2}\left(\mathrm{Fo}^{2}\right)+(0.0511 \cdot \mathrm{P})^{2}\right.$

$+0.4705 \cdot \mathrm{P}$ ]

where $\mathrm{P}=\left(\operatorname{Max}\left(\mathrm{Fo}^{2}, 0\right)+2 \mathrm{Fc}^{2}\right) / 3$

$136.4^{0}$

All non-hydrogen atoms

3927

298 
Supporting Information

Reflection/Parameter Ratio

Residuals: R1 (I>2.00s(I))

Residuals: R (All reflections)

Residuals: wR2 (All reflections)

Goodness of Fit Indicator

Flack Parameter $($ Friedel pairs $=1651)$

Max Shift/Error in Final Cycle

Maximum peak in Final Diff. Map

Minimum peak in Final Diff. Map
13.18

0.0569

0.0779

0.1268

1.045

$0.04(3)$

0.000

$0.87 \mathrm{e}^{-/} \AA^{3}$

$-0.55 \mathrm{e}^{-} / \AA^{3}$ 


\section{Chiral Amplification}

\section{General procedure for chiral amplification studies ( $40 \%$ ee of the catalyst)}

A pre-dried $10 \mathrm{~mL}$ test tube equipped with a magnetic stir bar was charged with diazocarbonyl compound $1(0.075 \mathrm{mmol}, 29.3 \mathrm{mg})$, which was subsequently dissolved in THF (0.05 M, $1.5 \mathrm{~mL}$ ) under argon gas atmosphere. The test tube was covered with aluminum foil to avoid light exposure, and to the stirred solution were added benzoic acid $(0.0075 \mathrm{mmol}, 10 \mathrm{~mol} \%, 0.92 \mathrm{mg})$ and [(S)TRIPAg $]_{2}(0.0026 \mathrm{mmol}, 1.3 \mathrm{~mol} \%)$ and $[(R) \text {-TRIPAg }]_{2}(0.00113 \mathrm{mmol}, 0.57$ mol \%) at room temperature. The reaction mixture was stirred for $24 \mathrm{~h}$ at the same temperature, and then passed through a short pad of $\mathrm{NH}$ silica gel to remove benzoic acid and $\mathrm{Ag}$ catalyst. The filtrate was concentrated by rotary evaporation under reduced pressure and purified by preparative thin layer chromatography (column condition; $n$-hexane/EtOAc, 1/1) to afford spirocyclic compound 3a with $34 \%$ ee.

\begin{tabular}{ll}
\hline $\begin{array}{l}\mathrm{ee}_{\mathrm{cat}} \\
(\% \text { ee })\end{array}$ & $\begin{array}{l}\mathrm{ee}_{\text {prod }} \\
(\% \text { ee })\end{array}$ \\
\hline 20 & 14 \\
40 & 34 \\
60 & 46 \\
80 & 66 \\
100 & 90 \\
\hline
\end{tabular}

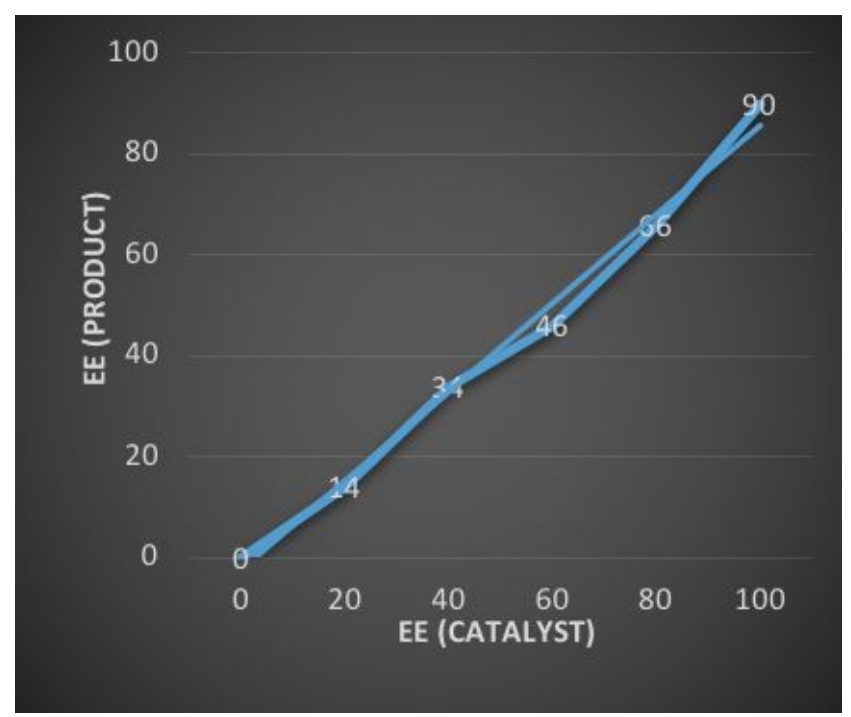




\section{3. $\quad$ Studies on the Additive Effect}

Table S1. Additive effect

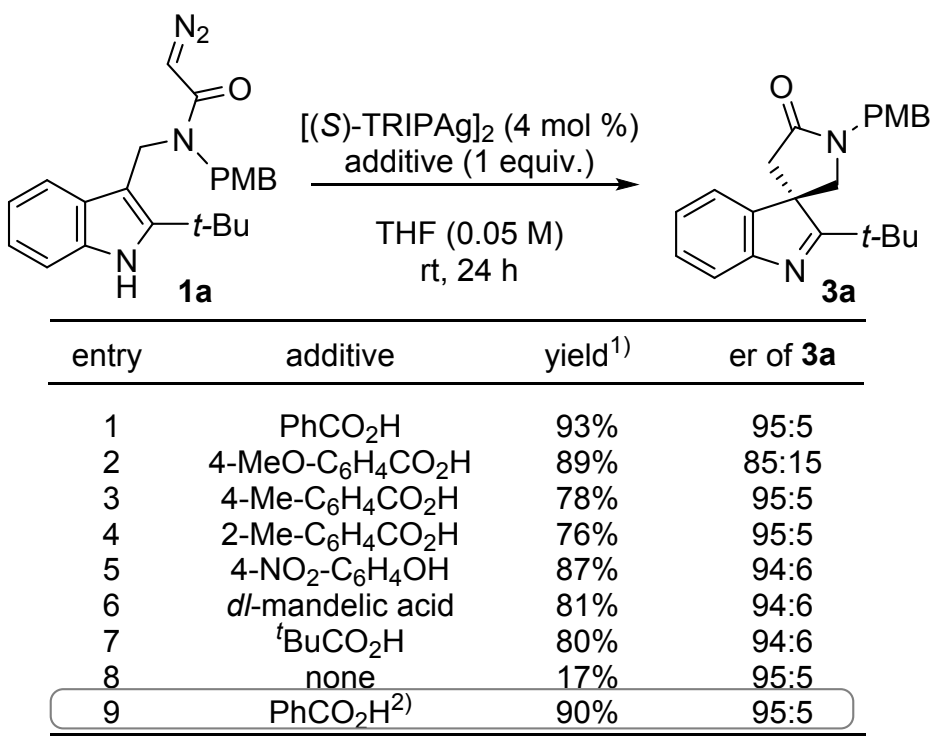

1) Determined by ${ }^{1} \mathrm{H}-\mathrm{NMR}$ analysis of crude mixture using $\mathrm{Ph}_{3} \mathrm{CH}$ as an internal standard.

2) $\mathrm{PhCO}_{2} \mathrm{H}: 10 \mathrm{~mol} \%$

Table S2. Different additive effect between phenol dearomatization and indole dearomatization $^{1}$

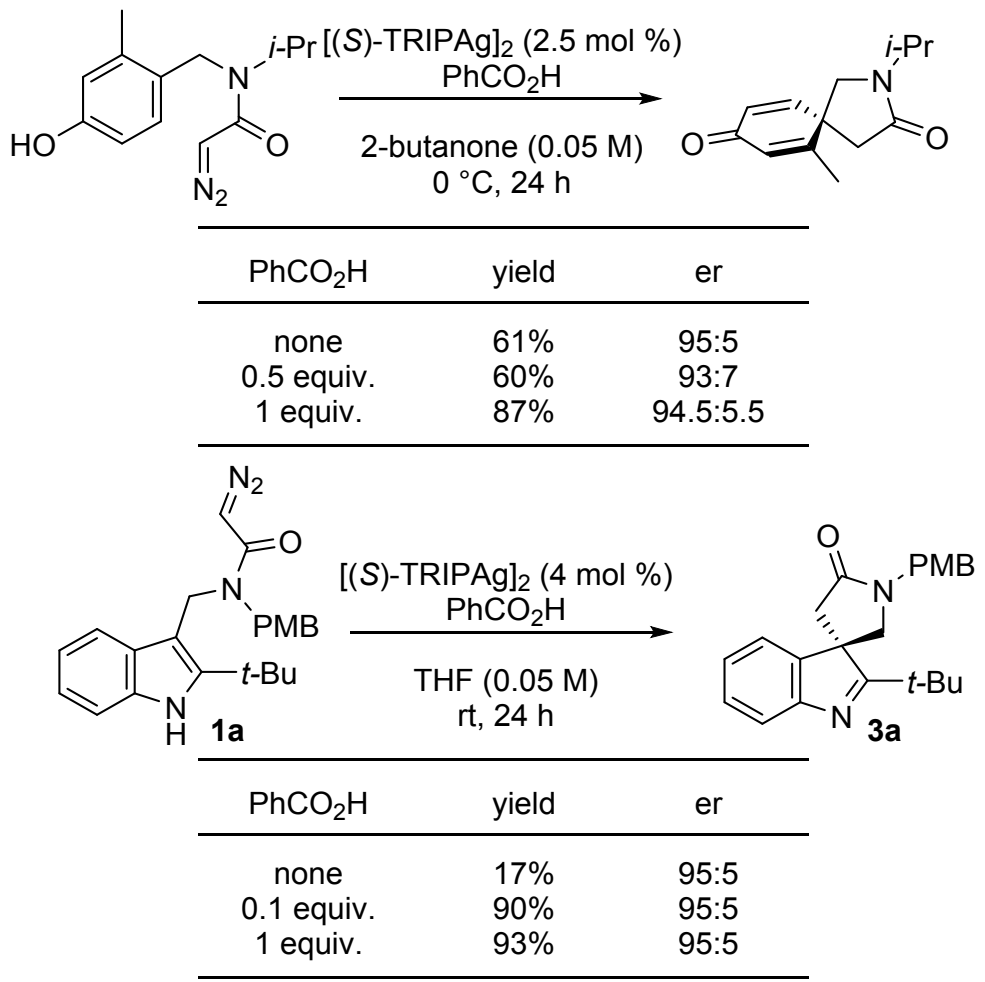




\section{4. $\quad$ Kinetic Studies}

\section{General procedure for kinetic experiments}

A pre-dried $10 \mathrm{~mL}$ test tube equipped with a magnetic stir bar was charged with diazocarbonyl compound $1 \mathrm{a}(0.0375-0.15 \mathrm{mmol})$ and triphenylmethane (as an internal standard, 1 equiv.), which were subsequently dissolved in THF (2.0 mL) under argon gas atmosphere. The test tube was covered with aluminum foil to avoid light exposure, and to the stirred solution were added benzoic acid (7.5$15.0 \mathrm{~mol} \%)$ and $[(S) \text {-TRIPAg }]_{2}(1.85-3.75 \mathrm{~mol} \%)$ at room temperature. The reaction mixture was stirred at the same temperature. Aliquots were taken at recorded times according to the following procedure. The reaction mixture $(0.2$ $\mathrm{mL}$ ) was withdrawn with a syringe, which was immediately passed through a short pad of $\mathrm{NH}$ silica gel. After the solvent was evaporated in vacuo, the crude residue was analyzed by ${ }^{1} \mathrm{H}$ NMR. Yields of the products were measured by comparison with internal standard. 
Initial Rate Kinetics for $[(S) \text {-TRIPAg }]_{2}$

$[(S) \text {-TRIPAg }]_{2}=0.94-1.875 \mathrm{mM},[1 \mathrm{a}]=0.05 \mathrm{M},[$ benzoic acid $]=5.0 \mathrm{mM}$.

Figure S-1. Initial Rate Kinetics $\left([(S) \text {-TRIPAg }]_{2}\right)$

\begin{tabular}{cccc}
\hline $\begin{array}{c}\left.\text { [[(S)-TRIPAg }{ }_{2}\right] \\
(\mathrm{M})\end{array}$ & $\begin{array}{c}\text { log } \\
{\left[[(\mathrm{S})-\mathrm{TRIPAg}]_{2}\right]}\end{array}$ & $\begin{array}{c}\text { Initial rate } \\
(\mathrm{M} / \mathbf{s})\end{array}$ & $\begin{array}{c}\text { log } \\
\text { (Initial rate) }\end{array}$ \\
\hline $1.875 \times 10^{-3}$ & -2.727 & $3.46 \times 10^{-3}$ & -2.465 \\
$1.565 \times 10^{-3}$ & -2.805 & $3.02 \times 10^{-3}$ & -2.520 \\
$1.25 \times 10^{-3}$ & -2.903 & $2.56 \times 10^{-3}$ & -2.592 \\
& & & \\
$0.94 \times 10^{-3}$ & -3.027 & $2.21 \times 10^{-3}$ & -2.656 \\
\hline
\end{tabular}

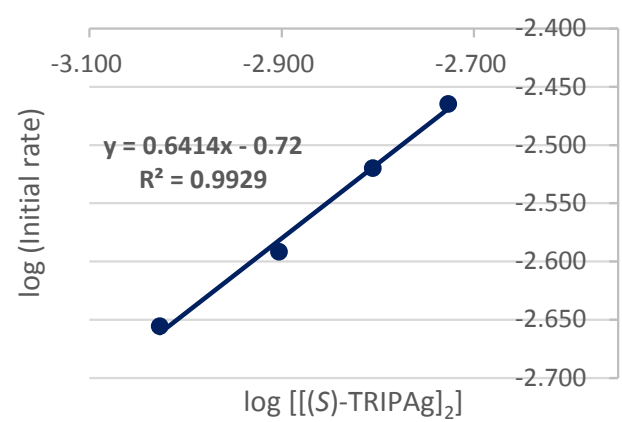

The order dependency of the reaction with regard to the $[(S) \text {-TRIPAg }]_{2}$ was determined to be 0.64. Detailed kinetics data are summarized in Figure S-2.

Figure S-2. Kinetics data for $[(S) \text {-TRIPAg }]_{2}$

\begin{tabular}{cc}
$\begin{array}{c}\left.[(\boldsymbol{S})-\mathrm{TRIPAg}]_{2}\right] \\
\mathbf{0 . 9 4} \times \mathbf{1 0}^{-3}\end{array}$ \\
\hline $\begin{array}{l}\text { Time } \\
(\mathbf{m i n})\end{array}$ & $\begin{array}{l}\text { Yield } \\
(\%)\end{array}$ \\
\hline 18 & 2.5 \\
25 & 4.2 \\
32 & 5.6 \\
\hline
\end{tabular}

\begin{tabular}{|c|c|}
\hline $\begin{array}{l}\text { Time } \\
\text { (min) }\end{array}$ & $\begin{array}{l}\begin{array}{l}\text { Yield } \\
(\%)\end{array} \\
\end{array}$ \\
\hline 12 & 2.5 \\
\hline 24 & 5.1 \\
\hline 36 & 7.3 \\
\hline
\end{tabular}

\begin{tabular}{|c|c|}
\hline $\begin{array}{l}\text { Time } \\
\text { (min) }\end{array}$ & $\begin{array}{l}\begin{array}{l}\text { Yield } \\
(\%)\end{array} \\
\end{array}$ \\
\hline 10 & 3.7 \\
\hline 15 & 4.4 \\
\hline 20 & 6.4 \\
\hline 30 & 9.5 \\
\hline
\end{tabular}

\begin{tabular}{cc}
$\begin{array}{c}\left.[(\mathbf{S})-\mathrm{TRIPAg}]_{2}\right] \\
\mathbf{1 . 8 7 5} \times \mathbf{1 0}^{-3}\end{array}$ \\
\hline $\begin{array}{c}\text { Time } \\
(\mathbf{m i n})\end{array}$ & $\begin{array}{c}\text { Yield } \\
(\%)\end{array}$ \\
\hline 8 & 2.4 \\
13 & 4.1 \\
18 & 6.1 \\
36 & 12 \\
\hline
\end{tabular}

[[(S)-TRIPAg $\left.]_{2}\right]$ Initial rate

(M)

$(\mathrm{M} / \mathrm{s})$

\begin{tabular}{ll}
\hline $1.875 \times 10^{-3}$ & $3.43 \times 10^{-3}$ \\
$1.565 \times 10^{-3}$ & $3.02 \times 10^{-3}$ \\
$1.25 \times 10^{-3}$ & $2.56 \times 10^{-3}$ \\
$0.94 \times 10^{-3}$ & $2.21 \times 10^{-3}$ \\
\hline
\end{tabular}

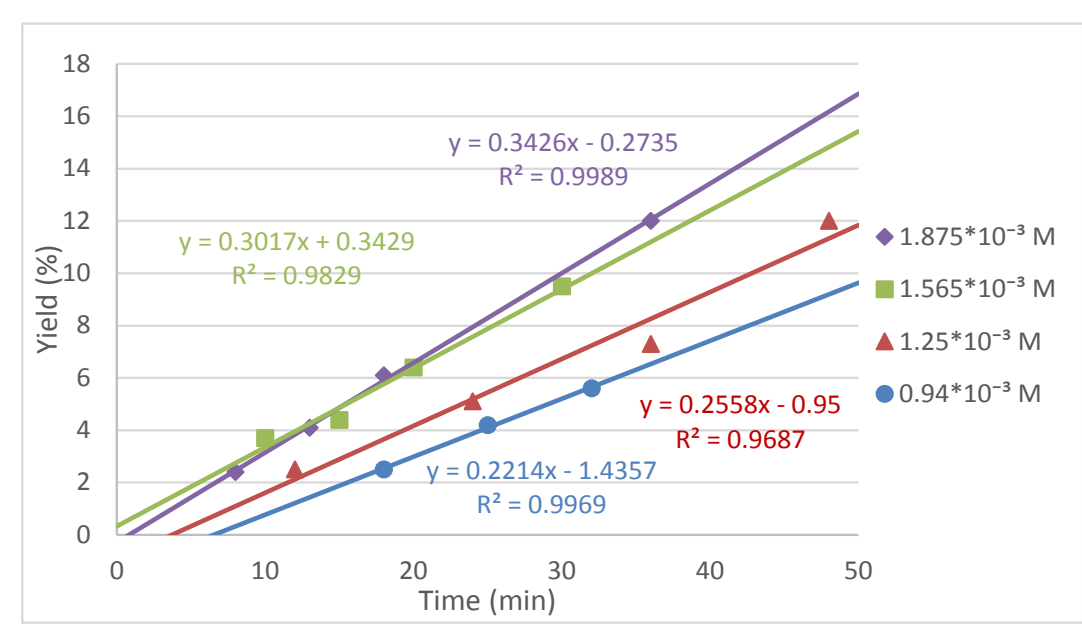


Initial Rate Kinetics for [BzOH]

$[(S) \text {-TRIPAg }]_{2}=1.25 \mathrm{mM},[1 \mathrm{a}]=0.05 \mathrm{M}$, [benzoic acid $]=3.75-7.5 \mathrm{mM}$.

Figure S-3. Initial Rate Kinetics (BzOH)

\begin{tabular}{cccc}
\hline $\begin{array}{c}\text { [BzOH] } \\
(\mathrm{M})\end{array}$ & $\begin{array}{c}\text { log } \\
{[\mathrm{BzOH}]}\end{array}$ & $\begin{array}{c}\text { Initial rate } \\
(\mathrm{M} / \mathrm{s})\end{array}$ & $\begin{array}{c}\text { log } \\
\text { (Initial rate) }\end{array}$ \\
\hline $7.50 \times 10^{-3}$ & -2.125 & $2.67 \times 10^{-3}$ & -2.573 \\
& & & \\
$6.25 \times 10^{-3}$ & -2.204 & $2.68 \times 10^{-3}$ & -2.572 \\
$5.00 \times 10^{-3}$ & -2.301 & $2.61 \times 10^{-3}$ & -2.583 \\
& & & \\
$3.75 \times 10^{-3}$ & -2.426 & $2.58 \times 10^{-3}$ & -2.588 \\
\hline
\end{tabular}

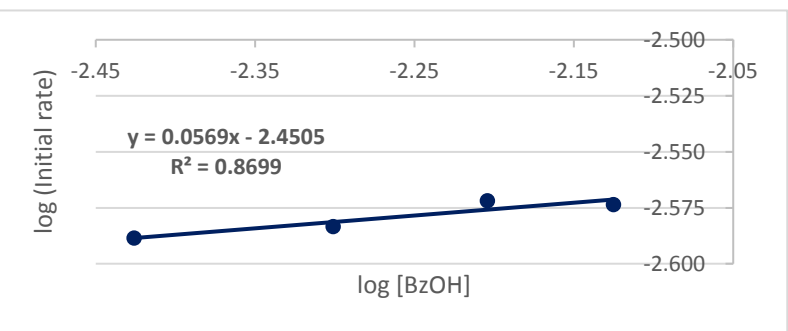

The order dependency of the reaction with regard to the $\mathrm{BzOH}$ was determined to be 0.057 . Namely, $\mathrm{BzOH}$ does not participate in the rate-determining step.

Detailed kinetics data are summarized in Figure S-4.

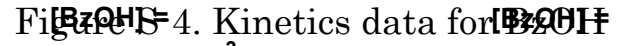

\begin{tabular}{cc}
\multicolumn{2}{c}{$\mathbf{3 . 7 5} \times \mathbf{1 0}^{-3}$} \\
\hline $\begin{array}{l}\text { Time } \\
(\mathbf{m i n})\end{array}$ & $\begin{array}{l}\text { Yield } \\
(\%)\end{array}$ \\
\hline 15 & 4.3 \\
28 & 7.6 \\
54 & 12 \\
67 & 15 \\
80 & 19 \\
110 & 28 \\
170 & 44 \\
\hline
\end{tabular}

\begin{tabular}{cc}
\multicolumn{2}{c}{$\mathbf{5 . 0 0} \times \mathbf{1 0}^{-3}$} \\
\hline $\begin{array}{l}\text { Time } \\
(\mathbf{m i n})\end{array}$ & $\begin{array}{l}\text { Yield } \\
(\%)\end{array}$ \\
\hline 12 & 2.5 \\
24 & 5.1 \\
36 & 7.3 \\
48 & 12 \\
90 & 21 \\
120 & 31 \\
150 & 38 \\
\hline
\end{tabular}

\begin{tabular}{cc}
$\begin{array}{l}{[\mathrm{BzOH}]=} \\
\mathbf{6 . 2 5} \times \mathbf{1 0}^{-3}\end{array}$ \\
\hline $\begin{array}{l}\text { Time } \\
(\mathbf{m i n})\end{array}$ & $\begin{array}{l}\text { Yield } \\
(\%)\end{array}$ \\
\hline 10 & 3.7 \\
20 & 5.1 \\
30 & 8.9 \\
40 & 12 \\
50 & 14 \\
60 & 17 \\
90 & 27
\end{tabular}

\begin{tabular}{cc}
$\begin{array}{c}{[\mathrm{BzOH}]=} \\
\mathbf{7 . 5 0} \times \mathbf{1 0}^{-3}\end{array}$ \\
\hline $\begin{array}{c}\text { Time } \\
(\mathbf{m i n})\end{array}$ & $\begin{array}{l}\text { Yield } \\
(\%)\end{array}$ \\
\hline 16 & 2.7 \\
24 & 4.6 \\
32 & 5.4 \\
40 & 6.7 \\
70 & 14 \\
130 & 33 \\
\hline
\end{tabular}

$150 \quad 40$

\begin{tabular}{cc}
\hline $\begin{array}{c}\text { [BzOH] } \\
(\mathrm{M})\end{array}$ & $\begin{array}{c}\text { Initial rate } \\
(\mathrm{M} / \mathrm{s})\end{array}$ \\
\hline $7.50 \times 10^{-3}$ & $2.67 \times 10^{-3}$ \\
$6.25 \times 10^{-3}$ & $2.68 \times 10^{-3}$ \\
$5.00 \times 10^{-3}$ & $2.61 \times 10^{-3}$ \\
$3.75 \times 10^{-3}$ & $2.58 \times 10^{-3}$ \\
\hline
\end{tabular}

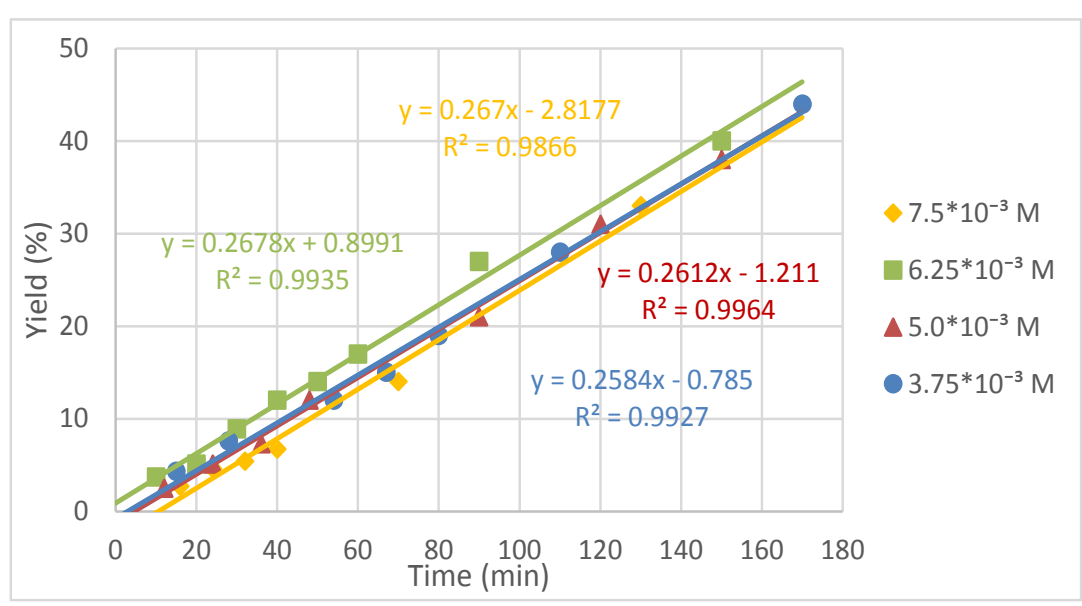


Initial Rate Kinetics for [1a]

$[(S)-\mathrm{TRIPAg}]_{2}=1.25 \mathrm{mM},[1 \mathrm{a}]=0.0188-0.075 \mathrm{M},[$ benzoic acid $]=5.0 \mathrm{mM}$.

Figure S-5. Initial Rate Kinetics (SM)

\begin{tabular}{cccc}
\hline $\begin{array}{c}\text { [SM] } \\
(\mathrm{M})\end{array}$ & $\begin{array}{c}\text { log } \\
{[\mathrm{SM}]}\end{array}$ & $\begin{array}{c}\text { Initial rate } \\
(\mathrm{M} / \mathbf{s})\end{array}$ & $\begin{array}{c}\text { log } \\
\text { (Initial rate) }\end{array}$ \\
\hline $7.50 \times 10^{-2}$ & -1.125 & $3.00 \times 10^{-3}$ & -2.523 \\
& & & \\
$5.00 \times 10^{-2}$ & -1.301 & $2.61 \times 10^{-3}$ & -2.583 \\
& & & \\
$2.50 \times 10^{-2}$ & -1.602 & $2.30 \times 10^{-3}$ & -2.638 \\
& & & \\
$1.88 \times 10^{-2}$ & -1.726 & $2.04 \times 10^{-3}$ & -2.690 \\
\hline
\end{tabular}

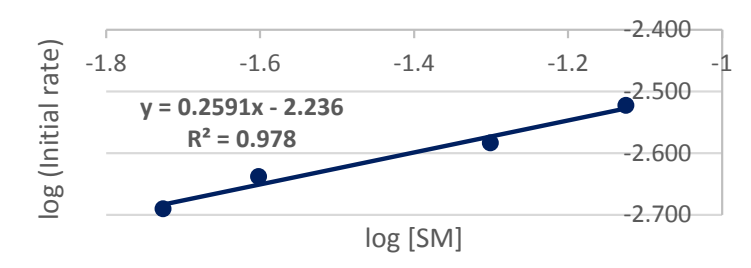

The order dependency of the reaction with regard to the SM was determined to be 0.26. Detailed kinetics data are summarized in Figure S-6.

Figure S-6. Kinetics data for SM

\begin{tabular}{cc}
$\begin{array}{c}\text { [SM] }= \\
\mathbf{1 . 8 8} \times \mathbf{1 0}^{-\mathbf{2}}\end{array}$ \\
\hline $\begin{array}{l}\text { Time } \\
(\mathbf{m i n})\end{array}$ & $\begin{array}{l}\text { Yield } \\
(\mathbf{\%})\end{array}$ \\
\hline 16 & 3.8 \\
30 & 5.8 \\
45 & 9.1 \\
60 & 13 \\
75 & 16 \\
105 & 21 \\
165 & 34 \\
\hline
\end{tabular}

\begin{tabular}{cc}
$\begin{array}{c}\text { [SM] }= \\
\mathbf{2 . 5 0} \times \mathbf{1 0}^{-\mathbf{2}}\end{array}$ \\
\hline $\begin{array}{c}\text { Time } \\
(\mathbf{m i n})\end{array}$ & $\begin{array}{l}\text { Yield } \\
(\%)\end{array}$ \\
\hline 16 & 4.6 \\
29 & 6.8 \\
42 & 11 \\
55 & 13 \\
70 & 16 \\
100 & 24 \\
160 & 40 \\
\hline
\end{tabular}

\begin{tabular}{cc}
$\begin{array}{c}{[\mathbf{S M}]=} \\
\mathbf{5 . 0 0} \times \mathbf{1 0}^{-2}\end{array}$ \\
\hline $\begin{array}{c}\text { Time } \\
(\mathbf{m i n})\end{array}$ & $\begin{array}{l}\text { Yield } \\
(\%)\end{array}$ \\
\hline 12 & 2.5 \\
24 & 5.1 \\
36 & 7.3 \\
48 & 12 \\
90 & 21 \\
120 & 31 \\
150 & 38 \\
\hline
\end{tabular}

\begin{tabular}{cc}
$\begin{array}{c}{[\mathrm{SM}]=} \\
\mathbf{7 . 5 0} \times \mathbf{1 0}^{-2}\end{array}$ \\
\hline $\begin{array}{l}\text { Time } \\
(\mathbf{m i n})\end{array}$ & $\begin{array}{c}\text { Yield } \\
(\%)\end{array}$ \\
\hline 8 & 4.4 \\
16 & 6.0 \\
24 & 6.6 \\
32 & 7.0 \\
40 & 10 \\
70 & 19 \\
100 & 32
\end{tabular}

\begin{tabular}{cc}
\hline $\begin{array}{cc}{[\mathrm{SM}]} \\
(\mathrm{M})\end{array}$ & $\begin{array}{c}\text { Initial rate } \\
(\mathrm{M} / \mathrm{s})\end{array}$ \\
\hline $7.50 \times 10^{-2}$ & $3.00 \times 10^{-3}$ \\
$5.00 \times 10^{-2}$ & $2.61 \times 10^{-3}$ \\
$2.50 \times 10^{-2}$ & $2.30 \times 10^{-3}$ \\
& \\
$1.88 \times 10^{-2}$ & $2.04 \times 10^{-3}$ \\
\hline
\end{tabular}

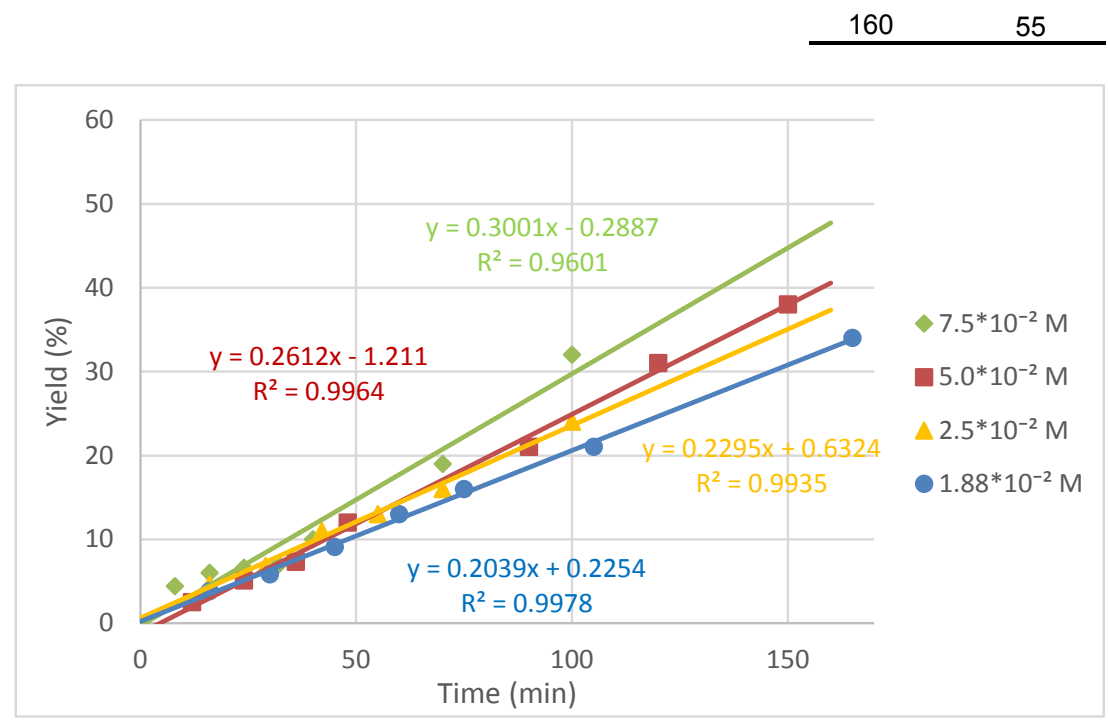




\section{5. $\quad$ Computational Details}

All the calculations were performed with Gaussian 16 program. ${ }^{[2]}$ The molecular structure optimizations were carried out using the hybrid density functional method based on Becke's three-parameter exchange function and the Lee-YangParr nonlocal correlation functional (B3LYP) ${ }^{[3]}$ and the LANL2DZ basis set for $\mathrm{Ag}$, and the $6-31 \mathrm{G}^{*}$ basis set for $\mathrm{H}, \mathrm{C}, \mathrm{N}, \mathrm{O}$, and $\mathrm{P}$. The vibrational frequencies were computed at the same level to check whether each optimized structure is at an energy minimum on the potential energy surfaces (no imaginary frequency) or a transition state (one imaginary frequency) and to evaluate its zero-point vibrational energy (ZPVE) and thermal corrections at $298.15 \mathrm{~K}$. The intrinsic reaction coordinate (IRC) method was used to track minimum energy paths from transition structures to the corresponding local minima. ${ }^{[4]}$ Single point energies were calculated at the $\mathrm{R} \omega \mathrm{B} 97 \mathrm{XD}$ level using $\mathrm{SDD}$ basis set ${ }^{[5]}$ for $\mathrm{Ag}$ and 6$311+\mathrm{G}^{* *}$ basis set for $\mathrm{H}, \mathrm{C}, \mathrm{N}, \mathrm{O}, \mathrm{P}, \mathrm{S}$ and $\mathrm{F}$ in THF solvent. 


\begin{tabular}{|c|c|c|c|}
\hline & $\begin{array}{c}\mathrm{E}\left(\mathrm{RB} 3 \mathrm{LYP} / 6-31 \mathrm{G}^{*}\right) \\
\text { (A.U.) }\end{array}$ & $\begin{array}{l}\text { Thermal } \\
\text { Correction to } \\
\text { Free Energy } \\
\text { (A.U.) }\end{array}$ & $\begin{array}{c}\text { Sum of Electronic } \\
\text { and Thermal Free } \\
\text { Energies } \\
\text { (A.U.) }\end{array}$ \\
\hline $\mathrm{CP0}$ & -3175.252380 & 0.910815 & -3174.341565 \\
\hline $\mathrm{TSO}_{A g^{-} C}$ & -3175.246192 & 0.914317 & -3174.331875 \\
\hline CP1 & -3175.247340 & 0.914075 & -3174.333265 \\
\hline $\mathrm{TS} 1_{\text {carbene }}$ & -3175.209172 & 0.908611 & -3174.300561 \\
\hline $\mathrm{CP} 2_{\text {carbene }}$ & -3065.705287 & 0.905468 & -3064.799819 \\
\hline $\mathrm{TS} 2_{\text {carbene }}$ & -3065.704951 & 0.906651 & -3064.798300 \\
\hline $\mathrm{TS} 1_{\text {carbenoid }}$ & -3175.225244 & 0.914413 & -3174.310831 \\
\hline CP3 & -3175.290089 & 0.911968 & -3174.378121 \\
\hline $\mathrm{CP} 4$ & -3254.150892 & 0.90682 & -3253.244071 \\
\hline $\mathrm{TS} 33_{\text {deargentation }}$ & -3254.118367 & 0.90739 & -3253.210977 \\
\hline CP5 & -3254.182297 & 0.907596 & -3253.274701 \\
\hline $\mathrm{BzOH}$ & -420.738228 & 0.083904 & -420.720575 \\
\hline $\mathrm{N}_{2}$ & -109.53698 & -0.012851 & -109.530627 \\
\hline $\mathrm{THF}$ & -232.36074 & 0.088709 & -232.356664 \\
\hline $\mathrm{TS}_{M A J O R}$ & -3175.225244 & 0.914413 & -3174.310831 \\
\hline $\mathrm{TS}_{M A J O R}$ & -3175.223351 & 0.912923 & -3174.310428 \\
\hline
\end{tabular}


Supporting Information

\begin{tabular}{|c|c|c|c|}
\hline & $\begin{array}{c}\mathrm{E}\left(\mathrm{R}_{W \mathrm{~B} 97 \mathrm{XD} /}\right. \\
\left.6-311+\mathrm{G}^{* *}\right) \\
(\mathrm{A} . \mathrm{U} .)\end{array}$ & $\begin{array}{l}\text { Thermal } \\
\text { Correction to } \\
\text { Free Energy } \\
\text { (A.U.) }\end{array}$ & $\begin{array}{c}\text { Sum of Electronic } \\
\text { and Thermal Free } \\
\text { Energies } \\
\text { (A.U.) }\end{array}$ \\
\hline CP0 & -3176.42315 & 0.910815 & -3175.512335 \\
\hline $\mathrm{TSO}_{A g-C}$ & -3176.421168 & 0.914317 & -3175.506851 \\
\hline $\mathrm{CP} 1$ & -3176.427765 & 0.914075 & -3175.51369 \\
\hline $\mathrm{TS} 1_{\text {carbene }}$ & -3176.395577 & 0.908611 & -3175.486966 \\
\hline $\mathrm{CP} 2_{\text {carbene }}$ & -3066.889106 & 0.905468 & -3065.983638 \\
\hline $\mathrm{TS} 2_{\text {carbene }}$ & -3066.887484 & 0.906651 & -3065.980833 \\
\hline $\mathrm{TS} 1_{\text {carbenoid }}$ & -3176.412889 & 0.914413 & -3175.498476 \\
\hline CP3 & -3176.484752 & 0.911968 & -3175.572784 \\
\hline $\mathrm{CP} 4$ & -3255.330706 & 0.90682 & -3254.423886 \\
\hline $\mathrm{TS} 3_{\text {deargentation }}$ & -3255.303994 & 0.90739 & -3254.396604 \\
\hline CP5 & -3255.361953 & 0.907596 & -3254.454357 \\
\hline $\mathrm{BzOH}$ & -420.804479 & 0.083904 & -420.720575 \\
\hline $\mathrm{N}_{2}$ & -109.517776 & -0.012851 & -109.530627 \\
\hline $\mathrm{THF}$ & -232.445373 & 0.088709 & -232.356664 \\
\hline $\mathrm{TS}_{M A J O R}$ & -3176.412889 & 0.914413 & -3175.498476 \\
\hline $\mathrm{TS}_{M A J O R}$ & -3176.409274 & 0.912923 & -3175.496351 \\
\hline
\end{tabular}




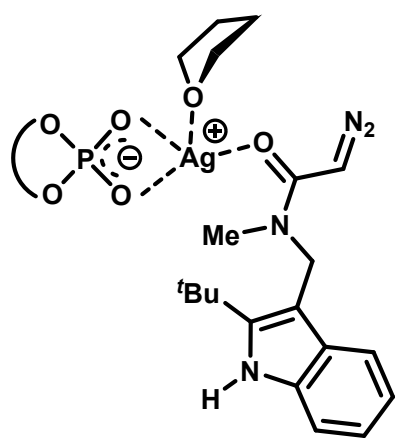

\section{CP0}

Zero-point correction $=$

Thermal correction to Energy=

Thermal correction to Enthalpy=

Thermal correction to Gibbs Free Energy=

Sum of electronic and zero-point Energies=

Sum of electronic and thermal Energies=

Sum of electronic and thermal Enthalpies=

Sum of electronic and thermal Free Energies=
1.025209 (Hartree/Particle)

1.092931

1.093875

0.910815

$-3174.227171$

$-3174.159449$

$-3174.158505$

$-3174.341565$

\section{Cartesian Coordinates}

$\begin{array}{lccc}\text { Atom } & \mathrm{X} & \mathrm{Y} & \mathrm{Z} \\ \mathrm{-} & & & \\ \mathrm{C} & 3.75276700 & 1.98841100 & 1.45655600 \\ \mathrm{C} & 5.11187800 & 2.33019100 & 1.46699600 \\ \mathrm{C} & 6.09353200 & 1.37825900 & 1.21827800 \\ \mathrm{C} & 5.77232300 & 0.03198200 & 1.00023700 \\ \mathrm{C} & 4.41568700 & -0.36018400 & 1.06217400 \\ \mathrm{C} & 3.43719100 & 0.64137500 & 1.23358800 \\ \mathrm{H} & 5.39216900 & 3.36412500 & 1.65047700 \\ \mathrm{C} & 3.98731800 & -1.78681000 & 0.92387500 \\ \mathrm{C} & 3.05732900 & -2.13591500 & -0.08018000 \\ \mathrm{C} & 4.47502500 & -2.80821100 & 1.76991200 \\ \mathrm{C} & 2.70039400 & -3.46247500 & -0.35862600 \\ \mathrm{C} & 3.26503700 & -4.45876900 & 0.44987900 \\ \mathrm{H} & 3.01760100 & -5.49840700 & 0.25170600\end{array}$


Supporting Information

\begin{tabular}{|c|c|c|c|}
\hline $\mathrm{O}$ & 2.52247600 & -1.12998600 & -0.86487900 \\
\hline $\mathrm{O}$ & 2.10445500 & 0.26318600 & 1.22691600 \\
\hline $\mathrm{P}$ & 1.39216000 & -0.10338200 & -0.22171600 \\
\hline $\mathrm{Ag}$ & -0.34377200 & 1.90103100 & -2.05753100 \\
\hline $\mathrm{O}$ & 0.08604600 & -0.76128200 & 0.08643800 \\
\hline $\mathrm{O}$ & 1.46907300 & 1.10742300 & -1.14609100 \\
\hline $\mathrm{H}$ & 7.13714200 & 1.68266100 & 1.18136300 \\
\hline $\mathrm{C}$ & -2.22444800 & -1.44214000 & -2.21278300 \\
\hline $\mathrm{H}$ & -1.96848000 & -2.36265200 & -1.71071100 \\
\hline $\mathrm{C}$ & -2.58176300 & -0.16953900 & -1.59101800 \\
\hline $\mathrm{O}$ & -2.47396100 & 0.89981100 & -2.25613600 \\
\hline $\mathrm{N}$ & -3.04022000 & -0.16017500 & -0.31671300 \\
\hline $\mathrm{C}$ & -3.14568600 & 1.14853500 & 0.32460100 \\
\hline $\mathrm{H}$ & -3.63973800 & 1.84650900 & -0.35159200 \\
\hline $\mathrm{H}$ & -3.73156900 & 1.04404400 & 1.23684800 \\
\hline $\mathrm{H}$ & -2.14993100 & 1.53933800 & 0.57619100 \\
\hline $\mathrm{N}$ & -1.60005900 & -1.33960600 & -4.57476700 \\
\hline $\mathrm{N}$ & -1.87692600 & -1.38897800 & -3.47352700 \\
\hline $\mathrm{C}$ & 4.10702700 & -4.13436600 & 1.50666700 \\
\hline $\mathrm{H}$ & 4.48934500 & -4.92439900 & 2.14924800 \\
\hline $\mathrm{C}$ & 2.70563400 & 3.03635500 & 1.67388000 \\
\hline $\mathrm{C}$ & 1.98862000 & 3.08275000 & 2.88961500 \\
\hline $\mathrm{C}$ & 2.46349500 & 4.00907400 & 0.68102100 \\
\hline $\mathrm{C}$ & 1.05066600 & 4.09936200 & 3.09247600 \\
\hline $\mathrm{C}$ & 1.51570200 & 5.01092300 & 0.92811700 \\
\hline $\mathrm{C}$ & 0.80049200 & 5.07822800 & 2.12595300 \\
\hline $\mathrm{H}$ & 0.50566100 & 4.12948200 & 4.03464400 \\
\hline $\mathrm{H}$ & 1.34088400 & 5.76537200 & 0.16223400 \\
\hline $\mathrm{C}$ & 1.76567200 & -3.83265000 & -1.46845700 \\
\hline $\mathrm{C}$ & 2.18982800 & -3.75347200 & -2.81345600 \\
\hline $\mathrm{C}$ & 0.47571300 & -4.31702500 & -1.17036500 \\
\hline $\mathrm{C}$ & 1.32256000 & -4.16809900 & -3.82850800 \\
\hline
\end{tabular}




\begin{tabular}{|c|c|c|c|}
\hline $\mathrm{C}$ & -0.35994500 & -4.72663700 & -2.21854600 \\
\hline $\mathrm{C}$ & 0.04295100 & -4.66238500 & -3.55497900 \\
\hline $\mathrm{H}$ & 1.66005900 & -4.10916000 & -4.86181600 \\
\hline $\mathrm{H}$ & -1.34843200 & -5.11693000 & -1.97952400 \\
\hline $\mathrm{C}$ & 5.34302200 & -2.53329300 & 2.97931700 \\
\hline $\mathrm{H}$ & 6.38976700 & -2.81069100 & 2.80247700 \\
\hline $\mathrm{H}$ & 5.32294200 & -1.48096900 & 3.27114200 \\
\hline $\mathrm{H}$ & 4.99636800 & -3.12892600 & 3.83159900 \\
\hline $\mathrm{C}$ & 2.21696200 & 2.05281400 & 3.97265700 \\
\hline $\mathrm{H}$ & 1.86352500 & 1.06531500 & 3.65377700 \\
\hline $\mathrm{H}$ & 3.28082700 & 1.95082800 & 4.21638800 \\
\hline $\mathrm{H}$ & 1.68367000 & 2.32608600 & 4.88907400 \\
\hline $\mathrm{C}$ & 3.56244400 & -3.23136100 & -3.17205900 \\
\hline $\mathrm{H}$ & 3.64626300 & -2.16286600 & -2.94266600 \\
\hline $\mathrm{H}$ & 4.35022300 & -3.74548500 & -2.60919500 \\
\hline $\mathrm{H}$ & 3.76424200 & -3.36605300 & -4.23951200 \\
\hline $\mathrm{C}$ & 6.89452900 & -0.92545000 & 0.66066000 \\
\hline $\mathrm{H}$ & 7.59835200 & -0.44681400 & -0.02951600 \\
\hline $\mathrm{H}$ & 7.46805500 & -1.21544000 & 1.55006700 \\
\hline $\mathrm{H}$ & 6.52787500 & -1.84032400 & 0.18979500 \\
\hline $\mathrm{C}$ & -0.19309800 & 6.18785900 & 2.38605400 \\
\hline $\mathrm{H}$ & -0.44750600 & 6.72236500 & 1.46433700 \\
\hline $\mathrm{H}$ & -1.12358100 & 5.80261000 & 2.82003100 \\
\hline $\mathrm{H}$ & 0.20729700 & 6.92731700 & 3.09261300 \\
\hline C & 3.17926200 & 3.97344600 & -0.65175400 \\
\hline $\mathrm{H}$ & 2.81289700 & 4.77162300 & -1.30697200 \\
\hline $\mathrm{H}$ & 4.26197700 & 4.10487300 & -0.54484900 \\
\hline $\mathrm{H}$ & 3.01272800 & 3.01150600 & -1.14847100 \\
\hline $\mathrm{C}$ & -0.03247400 & -4.36760700 & 0.25342600 \\
\hline $\mathrm{H}$ & -1.07552200 & -4.70170500 & 0.28169100 \\
\hline $\mathrm{H}$ & 0.55090600 & -5.05086800 & 0.88068800 \\
\hline 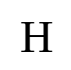 & 0.02853900 & -3.37399200 & 0.71154900 \\
\hline
\end{tabular}




\begin{tabular}{|c|c|c|c|}
\hline $\mathrm{C}$ & -0.87065700 & -5.10741700 & -4.67479700 \\
\hline $\mathrm{H}$ & -1.78353900 & -5.57113200 & -4.28633100 \\
\hline $\mathrm{H}$ & -1.17012700 & -4.26302300 & -5.30936400 \\
\hline $\mathrm{H}$ & -0.37713700 & -5.83723900 & -5.32821600 \\
\hline $\mathrm{C}$ & -3.98740900 & -1.33959100 & 1.67160100 \\
\hline $\mathrm{C}$ & -3.74415700 & -1.35020500 & 3.03838700 \\
\hline $\mathrm{C}$ & -5.41918300 & -1.34300200 & 1.48901200 \\
\hline $\mathrm{N}$ & -4.97110100 & -1.36367800 & 3.68830200 \\
\hline $\mathrm{C}$ & -6.00714300 & -1.35728300 & 2.77883900 \\
\hline $\mathrm{H}$ & -5.08353100 & -1.37324300 & 4.68869800 \\
\hline $\mathrm{C}$ & -6.25922400 & -1.33635100 & 0.36090200 \\
\hline $\mathrm{H}$ & -5.83834400 & -1.31581500 & -0.64066700 \\
\hline $\mathrm{C}$ & -7.63681100 & -1.34853500 & 0.54745300 \\
\hline $\mathrm{H}$ & -8.29552400 & -1.34521400 & -0.31685500 \\
\hline $\mathrm{C}$ & -8.19828900 & -1.36179700 & 1.84094600 \\
\hline $\mathrm{H}$ & -9.27885200 & -1.36907200 & 1.95484400 \\
\hline $\mathrm{C}$ & -7.39180900 & -1.36518600 & 2.97449600 \\
\hline $\mathrm{H}$ & -7.82327400 & -1.37558400 & 3.97230600 \\
\hline $\mathrm{C}$ & -2.44840400 & -1.39545900 & 3.84860700 \\
\hline $\mathrm{C}$ & -2.73964200 & -1.26018500 & 5.36034800 \\
\hline $\mathrm{H}$ & -3.24279400 & -0.31472200 & 5.59814900 \\
\hline $\mathrm{H}$ & -3.34695000 & -2.09114700 & 5.74209000 \\
\hline $\mathrm{H}$ & -1.79581400 & -1.27554200 & 5.91478400 \\
\hline $\mathrm{C}$ & -1.74084600 & -2.75300900 & 3.61900900 \\
\hline $\mathrm{H}$ & -1.46533400 & -2.89156400 & 2.57054000 \\
\hline $\mathrm{H}$ & -0.82126900 & -2.80449600 & 4.21449700 \\
\hline $\mathrm{H}$ & -2.38723200 & -3.58783200 & 3.91415200 \\
\hline $\mathrm{C}$ & -1.50232700 & -0.23885800 & 3.44545600 \\
\hline $\mathrm{H}$ & -1.14649400 & -0.31870800 & 2.41522700 \\
\hline $\mathrm{H}$ & -1.99685300 & 0.73171000 & 3.56881400 \\
\hline $\mathrm{H}$ & -0.61601000 & -0.24807200 & 4.09129100 \\
\hline $\mathrm{C}$ & -3.00802500 & -1.37784700 & 0.53716100 \\
\hline
\end{tabular}




$\begin{array}{lrrr}\mathrm{H} & -3.27511400 & -2.21771100 & -0.11189900 \\ \mathrm{H} & -1.98007100 & -1.52955100 & 0.87183600 \\ \mathrm{C} & -0.77035300 & 5.17192400 & -2.78134100 \\ \mathrm{O} & -1.21370900 & 3.83344400 & -3.11424300 \\ \mathrm{C} & -2.57614900 & 3.86173500 & -3.61028600 \\ \mathrm{C} & -3.13907300 & 5.19789500 & -3.13175700 \\ \mathrm{C} & -1.90358300 & 6.10989400 & -3.21501700 \\ \mathrm{H} & -0.59146000 & 5.21493900 & -1.70048200 \\ \mathrm{H} & 0.17462900 & 5.36257600 & -3.30024000 \\ \mathrm{H} & -2.54989600 & 3.80644000 & -4.70720000 \\ \mathrm{H} & -3.08437200 & 2.98019400 & -3.21744600 \\ \mathrm{H} & -3.97386600 & 5.54604100 & -3.74734100 \\ \mathrm{H} & -3.48829200 & 5.11547800 & -2.09561800 \\ \mathrm{H} & -1.75005900 & 6.44735900 & -4.24654100 \\ \mathrm{H} & -1.97434400 & 6.99573100 & -2.57692600\end{array}$

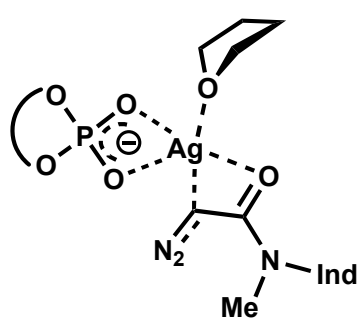

$\mathrm{TSO}_{A g-C}$

Zero-point correction $=$ 1.025642 (Hartree/Particle)

Thermal correction to Energy= 1.092404

Thermal correction to Enthalpy= 1.093348

Thermal correction to Gibbs Free Energy= 0.914317

Sum of electronic and zero-point Energies= $-3174.220550$

Sum of electronic and thermal Energies= $-3174.153788$

Sum of electronic and thermal Enthalpies= $-3174.152844$

Sum of electronic and thermal Free Energies $=\quad-3174.331875$

Cartesian Coordinates

Atom $\mathrm{X}$ $\mathrm{Y}$ $\mathrm{Z}$ 
$\begin{array}{lll}3.98941900 & -2.09828200 & -0.85502600\end{array}$

$\begin{array}{lll}5.35972500 & -2.34938900 & -0.70335100\end{array}$

C

$6.27463800-1.30688300-0.61454200$

C

$\begin{array}{lll}5.87627900 & 0.03161200 & -0.72776800\end{array}$

C

$\begin{array}{lll}4.51091900 & 0.31634500 & -0.95820700\end{array}$

C

$\begin{array}{lll}3.59618700 & -0.75731200 & -0.95633600\end{array}$

$\mathrm{H}$

$5.70046100 \quad-3.37898600 \quad-0.63296800$

$\begin{array}{lll}4.00927500 & 1.70905900 & -1.17370000\end{array}$

$\begin{array}{lll}2.99720700 & 2.21536000 & -0.33130900\end{array}$

C

$\begin{array}{lll}4.50257500 & 2.54372200 & -2.20178600\end{array}$

$\begin{array}{lll}2.54830800 & 3.54105200 & -0.38364000\end{array}$

C

$3.11425200 \quad 4.36401000-1.36715600$

$\mathrm{H}$

$\begin{array}{lll}2.79664500 & 5.40144100 & -1.42946000\end{array}$

$\mathrm{O}$

$\begin{array}{lll}2.45900600 & 1.37028200 & 0.62977200\end{array}$

$\mathrm{O}$

$2.24614300 \quad-0.47403200-1.11369900$

$$
1.41329300
$$

0.19062100

0.14414700

$\begin{array}{lll}-0.19421200 & -1.43293000 & 2.50946500\end{array}$

$\mathrm{O}$

$\mathrm{O}$

0.12247600

0.71229300

$-0.39621900$

$\mathrm{H}$

C

$\mathrm{H}$

C

$\mathrm{O}$

$\mathrm{N}$

$\begin{array}{lll}1.43232200 & -0.78268900 & 1.32492200\end{array}$

$\mathrm{H}$

$7.32565500-1.53018000$

$-0.44572400$

$-2.14351700$

1.33963200

1.68861400

$-1.50485400$

1.73508600

0.90975000

$-3.09624800$

0.21854800

1.56355600

$-3.43245500$

$-0.43359800$

2.57350400

$-3.56577800 \quad-0.04578000$

0.31098900

C

$\begin{array}{lll}-4.48062100 & -1.17049400 & 0.14220900\end{array}$

$\mathrm{H}$

$\begin{array}{lll}-4.77535200 & -1.50737100 & 1.13488500\end{array}$

$\mathrm{H}$

$\begin{array}{lll}-5.36670600 & -0.86008300 & -0.41621300\end{array}$

$\mathrm{H}$

$\begin{array}{lll}-4.00351600 & -1.99702900 & -0.39749500\end{array}$

$\mathrm{N}$

$-1.70011200$

$2.11282800 \quad 3.96451200$

$\mathrm{N}$

$\begin{array}{lll}-1.89223800 & 1.75252800 & 2.90002900\end{array}$ 


\begin{tabular}{|c|c|c|c|}
\hline $\mathrm{C}$ & 4.04587300 & 3.86664400 & -2.27074400 \\
\hline $\mathrm{H}$ & 4.42932700 & 4.51504100 & -3.05538400 \\
\hline $\mathrm{C}$ & 3.00975400 & -3.22906900 & -0.90896500 \\
\hline $\mathrm{C}$ & 2.41060200 & -3.58720500 & -2.13630900 \\
\hline $\mathrm{C}$ & 2.71317300 & -3.96586200 & 0.25673300 \\
\hline $\mathrm{C}$ & 1.52950100 & -4.67235700 & -2.17571100 \\
\hline $\mathrm{C}$ & 1.82147600 & -5.04290700 & 0.17201300 \\
\hline $\mathrm{C}$ & 1.22089400 & -5.41629200 & -1.03231400 \\
\hline $\mathrm{H}$ & 1.07844400 & -4.94781300 & -3.12775400 \\
\hline $\mathrm{H}$ & 1.59679300 & -5.60833300 & 1.07510000 \\
\hline $\mathrm{C}$ & 1.52091300 & 4.08090400 & 0.56231800 \\
\hline $\mathrm{C}$ & 1.86692400 & 4.34492800 & 1.90556600 \\
\hline $\mathrm{C}$ & 0.21977000 & 4.37478600 & 0.10265600 \\
\hline $\mathrm{C}$ & 0.91027500 & 4.90142600 & 2.76010400 \\
\hline $\mathrm{C}$ & -0.70735900 & 4.92964700 & 0.99445500 \\
\hline $\mathrm{C}$ & -0.38479700 & 5.20196800 & 2.32650500 \\
\hline $\mathrm{H}$ & 1.18752800 & 5.11047900 & 3.79186900 \\
\hline $\mathrm{H}$ & -1.70855100 & 5.15808700 & 0.63316900 \\
\hline $\mathrm{C}$ & 5.46767000 & 2.06242200 & -3.26388900 \\
\hline $\mathrm{H}$ & 6.48302300 & 2.44260400 & -3.09516400 \\
\hline $\mathrm{H}$ & 5.52375500 & 0.97247200 & -3.30758000 \\
\hline $\mathrm{H}$ & 5.15186700 & 2.42566600 & -4.24847700 \\
\hline $\mathrm{C}$ & 2.70572200 & -2.81859600 & -3.40471100 \\
\hline $\mathrm{H}$ & 2.29708300 & -1.80245100 & -3.35303100 \\
\hline $\mathrm{H}$ & 3.78370800 & -2.72248000 & -3.57849500 \\
\hline $\mathrm{H}$ & 2.26628600 & -3.31801500 & -4.27451400 \\
\hline $\mathrm{C}$ & 3.25114500 & 4.04086300 & 2.43308600 \\
\hline $\mathrm{H}$ & 3.43229200 & 2.96002200 & 2.46265600 \\
\hline $\mathrm{H}$ & 4.03151700 & 4.48133700 & 1.80160600 \\
\hline $\mathrm{H}$ & 3.37544800 & 4.43182000 & 3.44802900 \\
\hline $\mathrm{C}$ & 6.92480100 & 1.10864300 & -0.55009700 \\
\hline $\mathrm{H}$ & 7.60655500 & 0.83641100 & 0.2633700 \\
\hline
\end{tabular}




\begin{tabular}{|c|c|c|c|}
\hline $\mathrm{H}$ & 7.53804400 & 1.23466300 & -1.45116200 \\
\hline $\mathrm{H}$ & 6.48307800 & 2.07881900 & -0.31165200 \\
\hline $\mathrm{C}$ & 0.29079200 & -6.60565200 & -1.10845400 \\
\hline $\mathrm{H}$ & -0.08290000 & -6.88607800 & -0.11777400 \\
\hline $\mathrm{H}$ & -0.57401400 & -6.40003600 & -1.75003200 \\
\hline $\mathrm{H}$ & 0.79891800 & -7.48520200 & -1.52657800 \\
\hline $\mathrm{C}$ & 3.31864300 & -3.60376500 & 1.59558800 \\
\hline $\mathrm{H}$ & 2.90292600 & -4.23416500 & 2.38943000 \\
\hline $\mathrm{H}$ & 4.40686100 & -3.73612000 & 1.60503300 \\
\hline $\mathrm{H}$ & 3.11575800 & -2.55624000 & 1.84171200 \\
\hline $\mathrm{C}$ & -0.20059200 & 4.07686500 & -1.32009800 \\
\hline $\mathrm{H}$ & -1.25739500 & 4.32181700 & -1.47007500 \\
\hline $\mathrm{H}$ & 0.37989300 & 4.64959000 & -2.05234500 \\
\hline $\mathrm{H}$ & -0.05684900 & 3.01525500 & -1.54852600 \\
\hline $\mathrm{C}$ & -1.40623900 & 5.78353000 & 3.27744200 \\
\hline $\mathrm{H}$ & -2.23329500 & 6.25568000 & 2.73654100 \\
\hline $\mathrm{H}$ & -1.83562500 & 5.00645000 & 3.92369800 \\
\hline $\mathrm{H}$ & -0.95801400 & 6.53772000 & 3.93462700 \\
\hline $\mathrm{C}$ & -3.83992200 & 0.43507900 & -2.14604200 \\
\hline $\mathrm{C}$ & -3.39514300 & -0.29978900 & -3.23716400 \\
\hline $\mathrm{C}$ & -5.15502300 & 0.93193200 & -2.47180400 \\
\hline $\mathrm{N}$ & -4.38843700 & -0.26670900 & -4.20620000 \\
\hline $\mathrm{C}$ & -5.47004300 & 0.47098500 & -3.77506200 \\
\hline $\mathrm{H}$ & -4.32218100 & -0.72118400 & -5.10213000 \\
\hline C & -6.09595700 & 1.72296300 & -1.78810200 \\
\hline $\mathrm{H}$ & -5.88693000 & 2.08894700 & -0.78587800 \\
\hline C & -7.30069600 & 2.02867400 & -2.41157300 \\
\hline $\mathrm{H}$ & -8.03360000 & 2.64058200 & -1.89259500 \\
\hline $\mathrm{C}$ & -7.59101100 & 1.55724400 & -3.70825200 \\
\hline $\mathrm{H}$ & -8.54124900 & 1.81170500 & -4.16966000 \\
\hline $\mathrm{C}$ & -6.68017100 & 0.77153500 & -4.40765100 \\
\hline & -6.90228500 & 0.40806700 & -5.40790400 \\
\hline
\end{tabular}




\begin{tabular}{|c|c|c|c|}
\hline $\mathrm{C}$ & -2.07791400 & -1.02138600 & -3.52162600 \\
\hline $\mathrm{C}$ & -2.18623600 & -1.87639100 & -4.80474800 \\
\hline I & -2.99044900 & -2.61979300 & -4.73816200 \\
\hline IT & -2.34552600 & -1.26180900 & -5.70045700 \\
\hline $\mathrm{H}$ & -1.24810000 & -2.41937400 & -4.95816800 \\
\hline $\mathrm{C}$ & -0.94745600 & 0.01768900 & -3.72801200 \\
\hline $\mathrm{H}$ & -0.73483500 & 0.57455000 & -2.81243200 \\
\hline 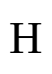 & -0.02145600 & -0.49334300 & -4.01908100 \\
\hline $\mathrm{H}$ & -1.20972200 & 0.72716200 & -4.52170600 \\
\hline $\mathrm{C}$ & -1.70653200 & -1.96720900 & -2.35617500 \\
\hline $\mathrm{H}$ & -1.54430600 & -1.42310400 & -1.42329000 \\
\hline $\mathrm{H}$ & -2.49057100 & -2.71709600 & -2.19518300 \\
\hline $\mathrm{H}$ & -0.77425800 & -2.49625000 & -2.58491100 \\
\hline $\mathrm{C}$ & -3.12138600 & 0.75605900 & -0.87056500 \\
\hline $\mathrm{H}$ & -3.30253700 & 1.81148100 & -0.63052000 \\
\hline $\mathrm{H}$ & -2.04286900 & 0.63762600 & -0.98515000 \\
\hline $\mathrm{C}$ & -2.49258800 & -3.38018000 & 3.56446500 \\
\hline $\mathrm{O}$ & -1.49718500 & -2.41498400 & 4.00586600 \\
\hline $\mathrm{C}$ & -2.01256300 & -1.65449400 & 5.14575500 \\
\hline $\mathrm{C}$ & -3.40943200 & -2.21907700 & 5.42175300 \\
\hline $\mathrm{C}$ & -3.34934700 & -3.62520900 & 4.80076700 \\
\hline $\mathrm{H}$ & -3.07557800 & -2.93271700 & 2.75461000 \\
\hline $\mathrm{H}$ & -1.94919300 & -4.25844800 & 3.20566700 \\
\hline $\mathrm{H}$ & -1.31317000 & -1.81734500 & 5.97271500 \\
\hline 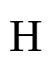 & -2.03620400 & -0.59876400 & 4.87579100 \\
\hline $\mathrm{H}$ & -3.64278300 & -2.23247200 & 6.49034800 \\
\hline $\mathrm{H}$ & -4.15614400 & -1.61170300 & 4.90322600 \\
\hline $\mathrm{H}$ & -2.85691800 & -4.33461600 & 5.47618300 \\
\hline & -4.33736200 & -4.01971500 & 4.54591200 \\
\hline
\end{tabular}




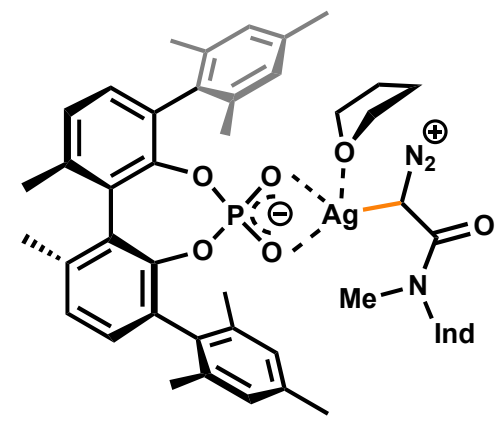

CP1

Zero-point correction $=$

Thermal correction to Energy=

Thermal correction to Enthalpy=

Thermal correction to Gibbs Free Energy=

Sum of electronic and zero-point Energies=

Sum of electronic and thermal Energies=

Sum of electronic and thermal Enthalpies=

Sum of electronic and thermal Free Energies=
1.025766 (Hartree/Particle)

1.093185

1.094129

0.914075

$-3174.221574$

$-3174.154156$

$-3174.153211$

$-3174.333265$

Cartesian Coordinates

Atom

$\mathrm{X}$ $\mathrm{Y}$

Z

C

$\begin{array}{lll}3.82259500 & -2.55221200 & -0.39137500\end{array}$

C

$\begin{array}{lll}5.15075200 & -2.93222400 & -0.15600200\end{array}$

C

$6.18018500-1.99887800-0.18811100$

$\mathrm{C}$

$5.94441200-0.65607900-0.51130200$

C

$4.62626300 \quad-0.25499200 \quad-0.82674100$

$\mathrm{C}$

$3.59159200-1.20502200 \quad-0.69826500$

$\mathrm{H}$

$\begin{array}{lll}5.36653700 & -3.97196300 & 0.07543500\end{array}$

C

$\begin{array}{lll}4.29438000 & 1.13662100 & -1.26374300\end{array}$

$\mathrm{C}$

$\begin{array}{lll}3.33507300 & 1.87475600 & -0.54030300\end{array}$

$\mathrm{C}$

$\begin{array}{lll}4.89252400 & 1.74332200 & -2.39110700\end{array}$

C

$\begin{array}{lll}3.03129400 & 3.21437800 & -0.81086400\end{array}$

$\mathrm{C}$

$\begin{array}{lll}3.69761600 & 3.80807800 & -1.89179400\end{array}$

$\mathrm{H}$

$\begin{array}{lll}3.49376600 & 4.84972600 & -2.12504000\end{array}$ 
Supporting Information

\begin{tabular}{|c|c|c|c|}
\hline $\mathrm{O}$ & 2.69525400 & 1.25079300 & 0.52441000 \\
\hline $\mathrm{O}$ & 2.28499100 & -0.79491800 & -0.93600600 \\
\hline $\mathrm{P}$ & 1.52814800 & 0.13943300 & 0.19044200 \\
\hline $\mathrm{Ag}$ & -0.53137700 & -0.92585500 & 2.37948700 \\
\hline $\mathrm{O}$ & 0.30462400 & 0.71759200 & -0.45019800 \\
\hline $\mathrm{O}$ & 1.39748600 & -0.63587300 & 1.50402500 \\
\hline $\mathrm{H}$ & 7.19440100 & -2.31250900 & 0.04842200 \\
\hline $\mathrm{C}$ & -1.86966100 & 1.64163200 & 1.59362200 \\
\hline $\mathrm{H}$ & -1.14253200 & 1.61894000 & 0.78519000 \\
\hline $\mathrm{C}$ & -3.26754700 & 1.15503000 & 1.59243600 \\
\hline $\mathrm{O}$ & -3.92215700 & 1.14412700 & 2.64752400 \\
\hline $\mathrm{N}$ & -3.76873100 & 0.71075000 & 0.39487000 \\
\hline $\mathrm{C}$ & -5.18348800 & 0.34528300 & 0.36273200 \\
\hline $\mathrm{H}$ & -5.51675400 & 0.20603900 & 1.38967300 \\
\hline $\mathrm{H}$ & -5.79011300 & 1.13212200 & -0.10259600 \\
\hline $\mathrm{H}$ & -5.32177000 & -0.57421700 & -0.21081400 \\
\hline $\mathrm{N}$ & -1.21850600 & 2.90470100 & 3.58776900 \\
\hline $\mathrm{N}$ & -1.51373300 & 2.32185900 & 2.65726700 \\
\hline $\mathrm{C}$ & 4.58402800 & 3.08001000 & -2.67687300 \\
\hline $\mathrm{H}$ & 5.04750500 & 3.55344600 & -3.53949500 \\
\hline $\mathrm{C}$ & 2.71941200 & -3.56285900 & -0.32794900 \\
\hline $\mathrm{C}$ & 2.13975700 & -4.04453400 & -1.52128200 \\
\hline $\mathrm{C}$ & 2.28782200 & -4.06918900 & 0.91649200 \\
\hline $\mathrm{C}$ & 1.13931000 & -5.02034600 & -1.44978900 \\
\hline $\mathrm{C}$ & 1.28027000 & -5.04172700 & 0.94148400 \\
\hline $\mathrm{C}$ & 0.69528700 & -5.53542100 & -0.22777600 \\
\hline $\mathrm{H}$ & 0.70656500 & -5.39664200 & -2.37547100 \\
\hline $\mathrm{H}$ & 0.95238200 & -5.43025900 & 1.90448000 \\
\hline $\mathrm{C}$ & 2.05282400 & 3.99871600 & 0.00742700 \\
\hline $\mathrm{C}$ & 2.41320700 & 4.44719300 & 1.29689900 \\
\hline $\mathrm{C}$ & 0.78939600 & 4.33221100 & -0.52455900 \\
\hline $\mathrm{C}$ & 1.50890400 & 5.22475000 & 2.02649300 \\
\hline
\end{tabular}




\begin{tabular}{|c|c|c|c|}
\hline $\mathrm{C}$ & -0.08502700 & 5.11260100 & 0.24263700 \\
\hline $\mathrm{C}$ & 0.25233300 & 5.57004800 & 1.51879100 \\
\hline $\mathrm{H}$ & 1.79721100 & 5.57438000 & 3.01630400 \\
\hline $\mathrm{H}$ & -1.05706900 & 5.37084600 & -0.17393900 \\
\hline $\mathrm{C}$ & 5.81569600 & 1.00242100 & -3.33432200 \\
\hline $\mathrm{H}$ & 6.86535000 & 1.28407500 & -3.18304700 \\
\hline $\mathrm{H}$ & 5.74241100 & -0.08115900 & -3.21726100 \\
\hline $\mathrm{H}$ & 5.56668800 & 1.24913600 & -4.37259600 \\
\hline $\mathrm{C}$ & 2.58547400 & -3.52743700 & -2.87075800 \\
\hline $\mathrm{H}$ & 2.31251700 & -2.47369400 & -3.00026100 \\
\hline $\mathrm{H}$ & 3.67308900 & -3.59490800 & -2.98915800 \\
\hline $\mathrm{H}$ & 2.12036800 & -4.09871100 & -3.68070400 \\
\hline $\mathrm{C}$ & 3.75748500 & 4.10418500 & 1.89896400 \\
\hline $\mathrm{H}$ & 3.83435400 & 3.03016000 & 2.10561400 \\
\hline $\mathrm{H}$ & 4.58220500 & 4.36235600 & 1.22439100 \\
\hline $\mathrm{H}$ & 3.91000400 & 4.64012700 & 2.84124900 \\
\hline $\mathrm{C}$ & 7.10920400 & 0.30900500 & -0.46000200 \\
\hline $\mathrm{H}$ & 7.73677400 & 0.09214700 & 0.41164900 \\
\hline $\mathrm{H}$ & 7.75150000 & 0.22097900 & -1.34519900 \\
\hline $\mathrm{H}$ & 6.78060800 & 1.34840600 & -0.39077700 \\
\hline $\mathrm{C}$ & -0.35201900 & -6.62496300 & -0.17656300 \\
\hline $\mathrm{H}$ & -0.89808800 & -6.61321900 & 0.77320400 \\
\hline $\mathrm{H}$ & -1.08166200 & -6.52221000 & -0.98782800 \\
\hline $\mathrm{H}$ & 0.10211200 & -7.62029300 & -0.27633100 \\
\hline $\mathrm{C}$ & 2.87626500 & -3.57053200 & 2.21816600 \\
\hline $\mathrm{H}$ & 2.38269500 & -4.04744000 & 3.07206600 \\
\hline $\mathrm{H}$ & 3.94849400 & -3.78571600 & 2.29244900 \\
\hline $\mathrm{H}$ & 2.75340600 & -2.48597900 & 2.30710700 \\
\hline $\mathrm{C}$ & 0.34847400 & 3.83834100 & -1.88504900 \\
\hline $\mathrm{H}$ & -0.65782100 & 4.20140200 & -2.11951900 \\
\hline $\mathrm{H}$ & 1.01799700 & 4.17326800 & -2.68518700 \\
\hline $\mathrm{H}$ & 0.33311300 & 2.74297800 & -1.90744900 \\
\hline
\end{tabular}




\begin{tabular}{|c|c|c|c|}
\hline $\mathrm{C}$ & -0.71496800 & 6.39468600 & 2.33711800 \\
\hline $\mathrm{H}$ & -1.49163800 & 6.84213000 & 1.70771100 \\
\hline $\mathrm{H}$ & -1.21732600 & 5.77908400 & 3.09485300 \\
\hline $\mathrm{H}$ & -0.20188000 & 7.20499700 & 2.86801700 \\
\hline $\mathrm{C}$ & -3.70602000 & 0.33404900 & -2.09473200 \\
\hline $\mathrm{C}$ & -3.45454500 & -0.90306200 & -2.67460600 \\
\hline $\mathrm{C}$ & -4.70856900 & 0.98710100 & -2.90338900 \\
\hline $\mathrm{N}$ & -4.26592400 & -1.02402900 & -3.79321500 \\
\hline $\mathrm{C}$ & -5.03855200 & 0.10436100 & -3.96275300 \\
\hline $\mathrm{H}$ & -4.27878100 & -1.82708700 & -4.40048500 \\
\hline $\mathrm{C}$ & -5.35491600 & 2.23531500 & -2.84217300 \\
\hline $\mathrm{H}$ & -5.12328600 & 2.94178500 & -2.04886700 \\
\hline $\mathrm{C}$ & -6.29425500 & 2.55976800 & -3.81425100 \\
\hline $\mathrm{H}$ & -6.79601700 & 3.52284000 & -3.77492600 \\
\hline $\mathrm{C}$ & -6.60887900 & 1.66077000 & -4.85388700 \\
\hline $\mathrm{H}$ & -7.34798000 & 1.94159200 & -5.59909900 \\
\hline $\mathrm{C}$ & -5.98483500 & 0.42083900 & -4.94242400 \\
\hline $\mathrm{H}$ & -6.22242100 & -0.27299000 & -5.74496700 \\
\hline $\mathrm{C}$ & -2.45632600 & -2.00833300 & -2.34052000 \\
\hline $\mathrm{C}$ & -2.79084700 & -3.30855400 & -3.10686200 \\
\hline $\mathrm{H}$ & -3.80821300 & -3.66157100 & -2.89722300 \\
\hline $\mathrm{H}$ & -2.67460900 & -3.19085300 & -4.19204600 \\
\hline $\mathrm{H}$ & -2.09461700 & -4.09676900 & -2.80193400 \\
\hline $\mathrm{C}$ & -1.03222900 & -1.55679700 & -2.74919300 \\
\hline $\mathrm{H}$ & -0.69835400 & -0.68172900 & -2.18594000 \\
\hline $\mathrm{H}$ & -0.31706400 & -2.36498300 & -2.55618000 \\
\hline $\mathrm{H}$ & -0.99705000 & -1.31445100 & -3.81801100 \\
\hline $\mathrm{C}$ & -2.48510600 & -2.34044400 & -0.83128000 \\
\hline $\mathrm{H}$ & -2.24252300 & -1.46946900 & -0.21744600 \\
\hline $\mathrm{H}$ & -3.47448000 & -2.70405200 & -0.52730100 \\
\hline $\mathrm{H}$ & -1.74767500 & -3.12188400 & -0.61278000 \\
\hline $\mathrm{C}$ & -3.06351300 & 0.97203500 & -0.89585900 \\
\hline
\end{tabular}




$\begin{array}{lrrr}\mathrm{H} & -3.03540600 & 2.05833800 & -1.06084600 \\ \mathrm{H} & -2.02683700 & 0.64697600 & -0.80100300 \\ \mathrm{C} & -3.32993700 & -2.40058600 & 3.14394400 \\ \mathrm{O} & -2.24198700 & -1.58496900 & 3.65109800 \\ \mathrm{C} & -2.68310800 & -0.82286100 & 4.82067800 \\ \mathrm{C} & -4.15230600 & -1.20220700 & 5.02562700 \\ \mathrm{C} & -4.26084400 & -2.57437800 & 4.33879500 \\ \mathrm{H} & -3.82045900 & -1.86911100 & 2.32134400 \\ \mathrm{H} & -2.89197100 & -3.33024100 & 2.77089300 \\ \mathrm{H} & -2.04080000 & -1.12369400 & 5.65539800 \\ \mathrm{H} & -2.55887800 & 0.23859700 & 4.60608700 \\ \mathrm{H} & -4.42487100 & -1.23178700 & 6.08450900 \\ \mathrm{H} & -4.78590500 & -0.47122800 & 4.51620300 \\ \mathrm{H} & -3.89550700 & -3.37361900 & 4.99420300 \\ \mathrm{H} & -5.28265800 & -2.81892700 & 4.03399100\end{array}$

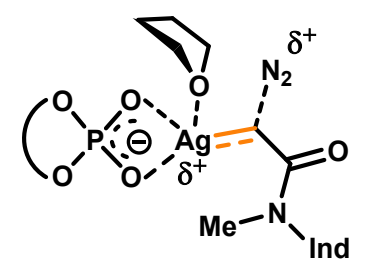

$\mathrm{TS} 1_{\text {carbene }}$

Zero-point correction $=$

Thermal correction to Energy=

Thermal correction to Enthalpy=

Thermal correction to Gibbs Free Energy=

Sum of electronic and zero-point Energies=

Sum of electronic and thermal Energies=

Sum of electronic and thermal Enthalpies=

Sum of electronic and thermal Free Energies=
1.021746 (Hartree/Particle)

1.089527

1.090471

0.908611

$-3174.187425$

$-3174.119644$

$-3174.118700$

$-3174.300561$

\section{Cartesian Coordinates}

Atom

$$
\text { X }
$$


$\begin{array}{lll}0.00624800 & -2.06270200 & 3.09482800\end{array}$

C

$\begin{array}{lll}0.45095800 & -2.82582200 & 4.18288000\end{array}$

C

$1.65046200-3.52613800$

4.12533100

$\mathrm{C}$

$2.43949000-3.53888100$

2.96722700

C

$\begin{array}{lll}1.98300100 & -2.83740000 & 1.82856800\end{array}$

C

$\begin{array}{lll}0.80157600 & -2.07610900 & 1.94164300\end{array}$

$\mathrm{H}$

$\begin{array}{lll}-0.14728400 & -2.84679200 & 5.09009800\end{array}$

$\begin{array}{lll}2.72534800 & -2.84499700 & 0.52936200\end{array}$

$\begin{array}{lll}3.14236700 & -1.61939500 & -0.03308900\end{array}$

$\begin{array}{lll}3.02534800 & -4.03666800 & -0.16780200\end{array}$

$3.95813600-1.53636200-1.16843200$

$3.81242900 \quad-3.95868700 \quad-1.32475400$

$\begin{array}{lll}4.29659600 & -2.74354300 & -1.79576700\end{array}$

$\mathrm{H}$

$4.93300800 \quad-2.71587100 \quad-2.67632900$

$\mathrm{O}$

$2.76300400-0.44142100$

0.59919500

$\mathrm{O}$

$0.38796100-1.34537800$

0.84031300

$\mathrm{Ag}$

1.19268500

0.04587700

0.44357100

Ag

$$
-0.58457300
$$

2.50566000

0.92951200

$\mathrm{O}$

$\mathrm{O}$

0.78216100

$0.38364600-0.95706600$

$$
1.02420100
$$

1.08834000

1.54567300

$\mathrm{H}$

$\begin{array}{lll}1.99404700 & -4.07115400 & 5.00161100\end{array}$

$\mathrm{H}$

$\begin{array}{lll}4.05067300 & -4.87427800 & -1.86155600\end{array}$

C

$-2.50796100$

2.72363100

0.12205600

$\mathrm{H}$

$-3.40243300$

2.42229200

0.67095100

C

$-2.61815800$

$2.77839900-1.37652400$

$\mathrm{O}$

$-2.54379500$

$3.85707700-1.96868300$

$\mathrm{N}$

$\begin{array}{lll}-2.70617600 & 1.55979300 & -1.98752200\end{array}$

C

$\begin{array}{lll}-2.35697800 & 1.48476600 & -3.40698100\end{array}$

$\mathrm{H}$

$-3.08558100$

$0.86733100-3.93738100$

$\mathrm{H}$

$-2.36111500$

$2.49636600-3.81058000$

$\mathrm{H}$

$\begin{array}{llll}-1.36144500 & 1.04011000 & -3.52512600\end{array}$ 


\begin{tabular}{|c|c|c|c|}
\hline $\mathrm{C}$ & -2.63034800 & 0.30779400 & -1.19838500 \\
\hline $\mathrm{H}$ & -1.57361800 & 0.06977800 & -1.03577800 \\
\hline $\mathrm{H}$ & -3.05749700 & 0.51025500 & -0.20777900 \\
\hline $\mathrm{C}$ & -3.29680400 & -0.87425800 & -1.84628500 \\
\hline $\mathrm{C}$ & -4.63868000 & -1.16911500 & -2.05795600 \\
\hline C & -2.52669900 & -1.96976300 & -2.39752100 \\
\hline $\mathrm{N}$ & -4.70583800 & -2.38261800 & -2.73040300 \\
\hline C & -3.44514600 & -2.89778900 & -2.94551000 \\
\hline $\mathrm{H}$ & -5.56396900 & -2.84298400 & -2.98818600 \\
\hline $\mathrm{C}$ & -1.14859900 & -2.25185800 & -2.46772100 \\
\hline $\mathrm{H}$ & -0.41524600 & -1.56934100 & -2.04705300 \\
\hline C & -0.74149100 & -3.43290000 & -3.07817900 \\
\hline $\mathrm{H}$ & 0.31892100 & -3.66337500 & -3.13324700 \\
\hline $\mathrm{C}$ & -1.67503300 & -4.33969500 & -3.62143300 \\
\hline $\mathrm{H}$ & -1.32246400 & -5.25341300 & -4.09248200 \\
\hline C & -3.04119500 & -4.08661100 & -3.56202600 \\
\hline $\mathrm{H}$ & -3.76404000 & -4.78445700 & -3.97732100 \\
\hline $\mathrm{C}$ & -5.94966300 & -0.44763700 & -1.76434500 \\
\hline $\mathrm{C}$ & -6.49184200 & 0.17671900 & -3.07476600 \\
\hline $\mathrm{H}$ & -5.79411300 & 0.92299900 & -3.46908300 \\
\hline $\mathrm{H}$ & -6.64376800 & -0.58370000 & -3.84925200 \\
\hline $\mathrm{H}$ & -7.45426700 & 0.67112700 & -2.89391800 \\
\hline $\mathrm{C}$ & -6.99471300 & -1.44793800 & -1.21004400 \\
\hline $\mathrm{H}$ & -6.62492100 & -1.95118200 & -0.30993600 \\
\hline $\mathrm{H}$ & -7.91638000 & -0.91524100 & -0.94967500 \\
\hline $\mathrm{H}$ & -7.27280700 & -2.21926100 & -1.93839500 \\
\hline $\mathrm{C}$ & -5.77498500 & 0.66934200 & -0.71981400 \\
\hline $\mathrm{H}$ & -5.37831600 & 0.27566200 & 0.22275800 \\
\hline $\mathrm{H}$ & -5.10900400 & 1.45429900 & -1.08656800 \\
\hline $\mathrm{H}$ & -6.74753000 & 1.12943000 & -0.51030400 \\
\hline $\mathrm{N}$ & -2.95608300 & 5.73948500 & 0.74855400 \\
\hline $\mathrm{N}$ & -3.01716600 & 4.67291100 & 0.46290000 \\
\hline
\end{tabular}




\begin{tabular}{|c|c|c|c|}
\hline $\mathrm{C}$ & 2.49590500 & -5.39126800 & 0.25135300 \\
\hline $\mathrm{H}$ & 3.27733300 & -6.01046500 & 0.70968800 \\
\hline $\mathrm{H}$ & 1.67330400 & -5.30963700 & 0.96516000 \\
\hline $\mathrm{H}$ & 2.12984500 & -5.93775000 & -0.62517700 \\
\hline $\mathrm{C}$ & 3.76484900 & -4.26832200 & 3.00899100 \\
\hline $\mathrm{H}$ & 4.24807900 & -4.11362900 & 3.98008700 \\
\hline $\mathrm{H}$ & 3.63909700 & -5.35115700 & 2.88399400 \\
\hline $\mathrm{H}$ & 4.44910400 & -3.92334000 & 2.23030400 \\
\hline $\mathrm{C}$ & 4.49219500 & -0.23688200 & -1.68870600 \\
\hline $\mathrm{C}$ & 5.55639400 & 0.39768000 & -1.01261300 \\
\hline $\mathrm{C}$ & 3.97945700 & 0.32675100 & -2.87678100 \\
\hline $\mathrm{C}$ & 6.09863700 & 1.57359600 & -1.54248500 \\
\hline $\mathrm{C}$ & 4.55187200 & 1.50509000 & -3.37022400 \\
\hline $\mathrm{C}$ & 5.61692400 & 2.14083300 & -2.72620400 \\
\hline $\mathrm{H}$ & 6.92569800 & 2.05187300 & -1.02045100 \\
\hline $\mathrm{H}$ & 4.15144100 & 1.93599200 & -4.28615700 \\
\hline $\mathrm{C}$ & -1.26552900 & -1.27641000 & 3.18550300 \\
\hline $\mathrm{C}$ & -2.42745700 & -1.73152200 & 2.52706400 \\
\hline $\mathrm{C}$ & -1.31581100 & -0.10053300 & 3.96659400 \\
\hline $\mathrm{C}$ & -3.61997300 & -1.01077800 & 2.67022800 \\
\hline $\mathrm{C}$ & -2.52775200 & 0.59212900 & 4.07647700 \\
\hline $\mathrm{C}$ & -3.69481700 & 0.15047700 & 3.44534500 \\
\hline $\mathrm{H}$ & -4.51731700 & -1.38231400 & 2.17762800 \\
\hline $\mathrm{H}$ & -2.56100900 & 1.49471300 & 4.68475100 \\
\hline $\mathrm{C}$ & 6.25248200 & 3.38186700 & -3.31163600 \\
\hline $\mathrm{H}$ & 7.06044400 & 3.12436700 & -4.01017500 \\
\hline $\mathrm{H}$ & 5.52431100 & 3.98193100 & -3.86856300 \\
\hline $\mathrm{H}$ & 6.68979000 & 4.01632500 & -2.53270200 \\
\hline $\mathrm{C}$ & 6.12079100 & -0.17096800 & 0.27047300 \\
\hline $\mathrm{H}$ & 5.38286600 & -0.13072200 & 1.08041100 \\
\hline $\mathrm{H}$ & 6.41258100 & -1.22134700 & 0.15441800 \\
\hline $\mathrm{H}$ & 7.00364600 & 0.39181800 & 0.59061300 \\
\hline
\end{tabular}


Supporting Information

\begin{tabular}{|c|c|c|c|}
\hline $\mathrm{C}$ & -2.40805800 & -2.98140200 & 1.67666800 \\
\hline $\mathrm{H}$ & -1.81400200 & -2.83398800 & 0.76752000 \\
\hline $\mathrm{H}$ & -1.96828700 & -3.82811000 & 2.21597200 \\
\hline $\mathrm{H}$ & -3.42115900 & -3.26162000 & 1.37114800 \\
\hline $\mathrm{C}$ & -0.08489900 & 0.43793900 & 4.66189100 \\
\hline $\mathrm{H}$ & -0.31906200 & 1.36393600 & 5.19811500 \\
\hline $\mathrm{H}$ & 0.32294300 & -0.27426300 & 5.38784300 \\
\hline $\mathrm{H}$ & 0.70296400 & 0.64968600 & 3.93102500 \\
\hline $\mathrm{C}$ & -5.00631800 & 0.87849200 & 3.64040500 \\
\hline $\mathrm{H}$ & -5.54253000 & 0.50355000 & 4.52284000 \\
\hline $\mathrm{H}$ & -4.85253600 & 1.95303400 & 3.79312000 \\
\hline $\mathrm{H}$ & -5.67423200 & 0.74932500 & 2.78120200 \\
\hline $\mathrm{C}$ & 2.80252500 & -0.28854900 & -3.59969900 \\
\hline $\mathrm{H}$ & 1.90867000 & -0.24501900 & -2.96697900 \\
\hline $\mathrm{H}$ & 2.59492300 & 0.25177300 & -4.52925300 \\
\hline $\mathrm{H}$ & 2.97647700 & -1.33998600 & -3.85370600 \\
\hline $\mathrm{C}$ & 2.24887600 & 3.98801800 & 2.04274700 \\
\hline $\mathrm{O}$ & 1.26568400 & 4.15471200 & 0.98831500 \\
\hline $\mathrm{C}$ & 1.93052900 & 4.16845300 & -0.30562400 \\
\hline $\mathrm{C}$ & 3.42052800 & 4.33518200 & -0.00637400 \\
\hline $\mathrm{C}$ & 3.55353900 & 3.61023100 & 1.34189600 \\
\hline $\mathrm{H}$ & 2.33281800 & 4.94106600 & 2.58252900 \\
\hline $\mathrm{H}$ & 1.89145500 & 3.21128500 & 2.72173700 \\
\hline $\mathrm{H}$ & 1.73318900 & 3.21749400 & -0.81497300 \\
\hline $\mathrm{H}$ & 1.49966100 & 4.98572000 & -0.89227100 \\
\hline $\mathrm{H}$ & 4.04779200 & 3.89710100 & -0.78758500 \\
\hline $\mathrm{H}$ & 3.67878000 & 5.39646300 & 0.09644800 \\
\hline $\mathrm{H}$ & 3.59135900 & 2.52719300 & 1.18761300 \\
\hline $\mathrm{H}$ & 4.43713700 & 3.91417300 & 1.91194600 \\
\hline
\end{tabular}




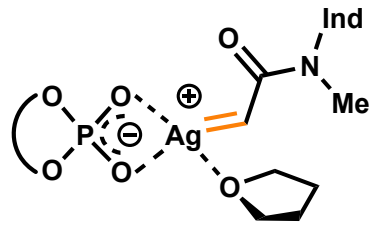

CP2 $2_{\text {carbene }}$

Zero-point correction $=$

Thermal correction to Energy=

Thermal correction to Enthalpy=

Thermal correction to Gibbs Free Energy=

Sum of electronic and zero-point Energies=

Sum of electronic and thermal Energies=

Sum of electronic and thermal Enthalpies=

Sum of electronic and thermal Free Energies=
1.014541 (Hartree/Particle)

1.080038

1.080982

$$
0.905468
$$

$-3064.690746$

$-3064.625249$

$-3064.624305$

$-3064.799819$

Cartesian Coordinates

$\begin{array}{llll}\text { Atom } & \mathrm{X} & \mathrm{Y} & \mathrm{Z}\end{array}$
C
3.48816600
2.84030300
$-0.75341700$
C
4.43045000
3.27997900
$-1.69388000$
C
5.27050900
$2.38351300 \quad-2.34317600$
C
5.25890800
$1.01661800 \quad-2.03612300$
C
4.37420800
$0.55235600-1.03709700$
$\mathrm{C}$
$\begin{array}{lll}3.45747900 & 1.46759800 & -0.47365400\end{array}$
$\mathrm{H}$
4.48323500
$4.34053000-1.92592100$
C
4.37675200
$-0.86503500$
$-0.56085200$
C
$\begin{array}{lll}3.18025000 & -1.60989600 & -0.61014700\end{array}$
C
$\begin{array}{lll}5.53117800 & -1.48164300 & -0.02679500\end{array}$
C
$\begin{array}{llll}3.10891800 & -2.96249400 & -0.24838100\end{array}$
C
$\begin{array}{llll}5.46510300 & -2.83389400 & 0.33400900\end{array}$
$\mathrm{C}$
$\begin{array}{llll}4.29004800 & -3.56509800 & 0.20449900\end{array}$
$\mathrm{H}$
$\begin{array}{lll}4.27165000 & -4.61666000 & 0.47898800\end{array}$
O
$\begin{array}{llll}2.04104500 & -0.98604300 & -1.08752000\end{array}$
O
2.52732400
0.99714400
0.43876100 


\begin{tabular}{|c|c|c|c|}
\hline $\mathrm{P}$ & 1.24658000 & 0.10265000 & -0.11861700 \\
\hline $\mathrm{Ag}$ & -1.38707800 & -1.43700900 & 0.88935600 \\
\hline $\mathrm{O}$ & 0.72158700 & -0.58508500 & 1.13281900 \\
\hline $\mathrm{O}$ & 0.32780600 & 0.86864400 & -1.02121300 \\
\hline $\mathrm{H}$ & 5.95517500 & 2.74646900 & -3.10660400 \\
\hline $\mathrm{H}$ & 6.35409800 & -3.31551400 & 0.73521700 \\
\hline $\mathrm{C}$ & -2.88100200 & -1.60264400 & -0.63650100 \\
\hline $\mathrm{H}$ & -3.90915200 & -1.96330600 & -0.60926100 \\
\hline $\mathrm{C}$ & -2.49772800 & -1.11038600 & -1.90770200 \\
\hline $\mathrm{O}$ & -2.21584900 & -2.32856000 & -2.16588000 \\
\hline $\mathrm{N}$ & -2.32426600 & -0.01801200 & -2.60791100 \\
\hline $\mathrm{C}$ & -1.44699200 & -0.05413600 & -3.78151900 \\
\hline $\mathrm{H}$ & -1.91907800 & 0.48150000 & -4.61062400 \\
\hline $\mathrm{H}$ & -0.49316700 & 0.41341400 & -3.51736500 \\
\hline $\mathrm{H}$ & -1.27597400 & -1.09303600 & -4.06626600 \\
\hline $\mathrm{C}$ & -2.68355700 & 1.31934300 & -2.06667400 \\
\hline $\mathrm{H}$ & -1.81489300 & 1.67833400 & -1.51114800 \\
\hline $\mathrm{H}$ & -2.80033700 & 1.95436100 & -2.95513100 \\
\hline $\mathrm{C}$ & -3.94808900 & 1.31535400 & -1.25721300 \\
\hline $\mathrm{C}$ & -4.18887500 & 1.94512900 & -0.04095900 \\
\hline $\mathrm{C}$ & -5.19873500 & 0.73658000 & -1.69765500 \\
\hline $\mathrm{N}$ & -5.52110100 & 1.74560700 & 0.28290700 \\
\hline $\mathrm{C}$ & -6.16152700 & 1.01451100 & -0.69353300 \\
\hline $\mathrm{H}$ & -5.95777700 & 2.09425800 & 1.12050900 \\
\hline $\mathrm{C}$ & -5.60849200 & 0.01811800 & -2.83770700 \\
\hline $\mathrm{H}$ & -4.90612500 & -0.20629900 & -3.63503000 \\
\hline $\mathrm{C}$ & -6.93062100 & -0.40085100 & -2.93528400 \\
\hline $\mathrm{H}$ & -7.25291600 & -0.95319200 & -3.81372900 \\
\hline $\mathrm{C}$ & -7.86339000 & -0.12230200 & -1.91587600 \\
\hline $\mathrm{H}$ & -8.88883000 & -0.46578300 & -2.01929900 \\
\hline $\mathrm{C}$ & -7.49080400 & 0.58995600 & -0.78104400 \\
\hline $\mathrm{H}$ & -8.20798100 & 0.81308300 & 0.00480300 \\
\hline
\end{tabular}




\begin{tabular}{|c|c|c|c|}
\hline $\mathrm{C}$ & -3.31110100 & 2.82353200 & 0.85073200 \\
\hline $\mathrm{C}$ & -4.10490900 & 3.33550300 & 2.07377300 \\
\hline $\mathrm{H}$ & -4.96662700 & 3.95000900 & 1.78330400 \\
\hline $\mathrm{H}$ & -4.45254600 & 2.51253400 & 2.71141000 \\
\hline $\mathrm{H}$ & -3.45352300 & 3.96447100 & 2.68876100 \\
\hline $\mathrm{C}$ & -2.08588900 & 2.04137400 & 1.37627000 \\
\hline $\mathrm{H}$ & -1.42494100 & 1.69771300 & 0.57722800 \\
\hline $\mathrm{H}$ & -1.48914100 & 2.68978800 & 2.02929200 \\
\hline $\mathrm{H}$ & -2.40123300 & 1.16928100 & 1.96109200 \\
\hline C & -2.83810600 & 4.05770100 & 0.04452200 \\
\hline $\mathrm{H}$ & -2.21904800 & 3.77203100 & -0.81002300 \\
\hline $\mathrm{H}$ & -3.69189100 & 4.63565600 & -0.32852400 \\
\hline $\mathrm{H}$ & -2.22975800 & 4.70932500 & 0.68180300 \\
\hline $\mathrm{C}$ & 6.82655900 & -0.73761500 & 0.21757200 \\
\hline $\mathrm{H}$ & 7.57363800 & -0.95134400 & -0.55720400 \\
\hline $\mathrm{H}$ & 6.68085300 & 0.34466300 & 0.24645900 \\
\hline $\mathrm{H}$ & 7.26238200 & -1.04803500 & 1.17385900 \\
\hline $\mathrm{C}$ & 6.16485800 & 0.09675300 & -2.82661200 \\
\hline $\mathrm{H}$ & 6.12785600 & 0.35898900 & -3.89024300 \\
\hline $\mathrm{H}$ & 7.21264900 & 0.18678700 & -2.51341900 \\
\hline $\mathrm{H}$ & 5.87664800 & -0.95203100 & -2.72678400 \\
\hline $\mathrm{C}$ & 1.83215600 & -3.73929000 & -0.32242400 \\
\hline $\mathrm{C}$ & 1.30902600 & -4.13239700 & -1.57304500 \\
\hline $\mathrm{C}$ & 1.16026100 & -4.10390000 & 0.86330200 \\
\hline $\mathrm{C}$ & 0.12386700 & -4.87138000 & -1.61652800 \\
\hline $\mathrm{C}$ & -0.02959500 & -4.83896100 & 0.77274300 \\
\hline $\mathrm{C}$ & -0.56927500 & -5.22721000 & -0.45639200 \\
\hline $\mathrm{H}$ & -0.27410900 & -5.16803400 & -2.58502500 \\
\hline $\mathrm{H}$ & -0.54204200 & -5.12267500 & 1.69103400 \\
\hline $\mathrm{C}$ & 2.59236200 & 3.83067000 & -0.07599700 \\
\hline $\mathrm{C}$ & 2.83623300 & 4.19751100 & 1.26502200 \\
\hline $\mathrm{C}$ & 1.54708700 & 4.45017700 & -0.79249800 \\
\hline
\end{tabular}




\begin{tabular}{|c|c|c|c|}
\hline $\mathrm{C}$ & 2.04683600 & 5.18919000 & 1.85618200 \\
\hline $\mathrm{C}$ & 0.78595900 & 5.44263600 & -0.16377000 \\
\hline C & 1.02314800 & 5.83393400 & 1.15649300 \\
\hline 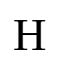 & 2.24455400 & 5.46999800 & 2.88939200 \\
\hline & -0.01493700 & 5.92156300 & -0.72447300 \\
\hline $\mathrm{C}$ & -1.87230200 & -5.98771900 & -0.53751600 \\
\hline $\mathrm{H}$ & -1.80831300 & -6.82326700 & -1.24477300 \\
\hline $\mathrm{H}$ & -2.16255300 & -6.39346500 & 0.43777200 \\
\hline $\mathrm{H}$ & -2.68487400 & -5.33501400 & -0.88250500 \\
\hline $\mathrm{C}$ & 2.00574100 & -3.76247400 & -2.86275900 \\
\hline $\mathrm{H}$ & 1.95140600 & -2.68191700 & -3.03930800 \\
\hline $\mathrm{H}$ & 3.06802100 & -4.03234300 & -2.84234800 \\
\hline $\mathrm{H}$ & 1.54233100 & -4.26978800 & -3.71519200 \\
\hline $\mathrm{C}$ & 3.93230100 & 3.53771500 & 2.07103900 \\
\hline $\mathrm{H}$ & 3.70721400 & 2.47936200 & 2.24686200 \\
\hline $\mathrm{H}$ & 4.89748300 & 3.57977800 & 1.55282900 \\
\hline $\mathrm{H}$ & 4.04859400 & 4.02613700 & 3.04403900 \\
\hline $\mathrm{C}$ & 1.20898900 & 4.03024000 & -2.20594800 \\
\hline $\mathrm{H}$ & 0.35315200 & 4.60046900 & -2.58375000 \\
\hline $\mathrm{H}$ & 2.04498300 & 4.18604800 & -2.89721500 \\
\hline $\mathrm{H}$ & 0.95763500 & 2.96360900 & -2.23100600 \\
\hline $\mathrm{C}$ & 0.21952700 & 6.94142000 & 1.80000600 \\
\hline $\mathrm{H}$ & 0.09150200 & 6.77299300 & 2.87537100 \\
\hline $\mathrm{H}$ & 0.71480400 & 7.91524100 & 1.68350000 \\
\hline $\mathrm{H}$ & -0.77570700 & 7.03071700 & 1.35005400 \\
\hline $\mathrm{C}$ & 1.68118300 & -3.69281000 & 2.22335000 \\
\hline $\mathrm{H}$ & 1.75765300 & -2.60194400 & 2.28813400 \\
\hline $\mathrm{H}$ & 1.00713100 & -4.04223200 & 3.01348100 \\
\hline $\mathrm{H}$ & 2.67533000 & -4.10491800 & 2.42930800 \\
\hline $\mathrm{C}$ & -1.98745000 & -2.39526800 & 4.22676200 \\
\hline $\mathrm{O}$ & -1.52544800 & -1.31383700 & 3.3919370 \\
\hline & -0.53535700 & -0.52327000 & 4.09705100 \\
\hline
\end{tabular}




$\begin{array}{llll}\mathrm{C} & -0.06900500 & -1.40611400 & 5.25326700 \\ \mathrm{C} & -1.35572300 & -2.17010000 & 5.60660700 \\ \mathrm{H} & -3.08313300 & -2.38248100 & 4.24417800 \\ \mathrm{H} & -1.65895200 & -3.34438600 & 3.78358800 \\ \mathrm{H} & 0.24149000 & -0.26026900 & 3.37776200 \\ \mathrm{H} & -1.01785100 & 0.39307900 & 4.46627700 \\ \mathrm{H} & 0.70891000 & -2.09856000 & 4.91211800 \\ \mathrm{H} & 0.33195900 & -0.82578700 & 6.08994400 \\ \mathrm{H} & -1.17423600 & -3.10871700 & 6.13922800 \\ \mathrm{H} & -2.00811800 & -1.54777700 & 6.23077300\end{array}$

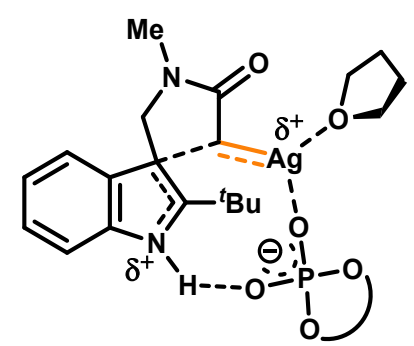

TS2 ${ }_{\text {carbene }}$

Zero-point correction $=$ 1.014133 (Hartree/Particle)

Thermal correction to Energy= 1.078885

Thermal correction to Enthalpy= 1.079830

Thermal correction to Gibbs Free Energy= 0.906651

Sum of electronic and zero-point Energies= $-3064.690818$

Sum of electronic and thermal Energies= -3064.626066

Sum of electronic and thermal Enthalpies= $-3064.625122$

Sum of electronic and thermal Free Energies $=\quad-3064.798300$

Cartesian Coordinates

$\begin{array}{llll}\text { Atom } & \mathrm{X} & \mathrm{Y} & \mathrm{Z}\end{array}$

$\begin{array}{llll}\mathrm{C} & 3.54656200 & 2.77270400 & -0.80099900 \\ \mathrm{C} & 4.53206200 & 3.17526400 & -1.71320200 \\ \mathrm{C} & 5.40155500 & 2.25416100 & -2.28457600\end{array}$




\begin{tabular}{|c|c|c|c|}
\hline $\mathrm{C}$ & 5.37401200 & 0.90062100 & -1.92396200 \\
\hline $\mathrm{C}$ & 4.44264000 & 0.47566500 & -0.94989900 \\
\hline $\mathrm{C}$ & 3.50263300 & 1.41276800 & -0.46680200 \\
\hline $\mathrm{H}$ & 4.59543300 & 4.22540500 & -1.98631300 \\
\hline $\mathrm{C}$ & 4.41683500 & -0.92452800 & -0.42544100 \\
\hline $\mathrm{C}$ & 3.22159600 & -1.66784200 & -0.50847100 \\
\hline $\mathrm{C}$ & 5.54211700 & -1.52908500 & 0.17985100 \\
\hline $\mathrm{C}$ & 3.12767600 & -3.01073000 & -0.11714300 \\
\hline $\mathrm{C}$ & 5.45329300 & -2.87054400 & 0.57470000 \\
\hline $\mathrm{C}$ & 4.28364500 & -3.60328200 & 0.40783800 \\
\hline $\mathrm{H}$ & 4.24937600 & -4.64743600 & 0.70773300 \\
\hline $\mathrm{O}$ & 2.10599100 & -1.05349500 & -1.05157300 \\
\hline $\mathrm{O}$ & 2.53188100 & 0.98091000 & 0.42240600 \\
\hline $\mathrm{P}$ & 1.27688500 & 0.05951800 & -0.14672800 \\
\hline $\mathrm{Ag}$ & -1.42615400 & -1.24843400 & 0.90033400 \\
\hline $\mathrm{O}$ & 0.72031100 & -0.58722600 & 1.11619000 \\
\hline $\mathrm{O}$ & 0.37300300 & 0.78540400 & -1.09537800 \\
\hline $\mathrm{H}$ & 6.12162300 & 2.58657900 & -3.02906500 \\
\hline $\mathrm{H}$ & 6.32036400 & -3.34286000 & 1.03122000 \\
\hline $\mathrm{C}$ & -2.99998200 & -1.47186300 & -0.50463400 \\
\hline $\mathrm{H}$ & -4.02964900 & -1.81424400 & -0.39799500 \\
\hline $\mathrm{C}$ & -2.60855300 & -1.29074800 & -1.86540700 \\
\hline $\mathrm{O}$ & -2.37813000 & -2.51323500 & -2.07824600 \\
\hline $\mathrm{N}$ & -2.41653800 & -0.23945800 & -2.64211700 \\
\hline $\mathrm{C}$ & -1.57156600 & -0.36348300 & -3.82865500 \\
\hline $\mathrm{H}$ & -2.04903800 & 0.13131800 & -4.68051400 \\
\hline $\mathrm{H}$ & -0.59845100 & 0.09526800 & -3.62274700 \\
\hline $\mathrm{H}$ & -1.43320600 & -1.42242600 & -4.05143500 \\
\hline $\mathrm{C}$ & -2.71650500 & 1.12290600 & -2.14616600 \\
\hline $\mathrm{H}$ & -1.81829700 & 1.49625000 & -1.64827700 \\
\hline $\mathrm{H}$ & -2.87684100 & 1.72884900 & -3.04845900 \\
\hline $\mathrm{C}$ & -3.93816300 & 1.16615100 & -1.26753300 \\
\hline
\end{tabular}




\begin{tabular}{|c|c|c|c|}
\hline $\mathrm{C}$ & -4.12788900 & 1.90527400 & -0.09906900 \\
\hline $\mathrm{C}$ & -5.21920100 & 0.58175400 & -1.61544800 \\
\hline $\mathrm{N}$ & -5.44563500 & 1.75058100 & 0.28924500 \\
\hline $\mathrm{C}$ & -6.13513400 & 0.95261900 & -0.59886300 \\
\hline $\mathrm{H}$ & -5.84219600 & 2.16610600 & 1.11654100 \\
\hline $\mathrm{C}$ & -5.68857500 & -0.20834600 & -2.68262200 \\
\hline $\mathrm{H}$ & -5.02451400 & -0.50269800 & -3.48934800 \\
\hline $\mathrm{C}$ & -7.02033600 & -0.60831800 & -2.69361300 \\
\hline $\mathrm{H}$ & -7.38828000 & -1.21578600 & -3.51584100 \\
\hline $\mathrm{C}$ & -7.90419000 & -0.24106900 & -1.65918000 \\
\hline $\mathrm{H}$ & -8.93800000 & -0.57281300 & -1.69415500 \\
\hline $\mathrm{C}$ & -7.47251000 & 0.54565300 & -0.59675200 \\
\hline $\mathrm{H}$ & -8.15146100 & 0.83975500 & 0.19953200 \\
\hline $\mathrm{C}$ & -3.22426900 & 2.87937200 & 0.65788700 \\
\hline $\mathrm{C}$ & -3.96715800 & 3.49621200 & 1.86463800 \\
\hline $\mathrm{H}$ & -4.85249000 & 4.06966700 & 1.56152100 \\
\hline $\mathrm{H}$ & -4.26779500 & 2.73389600 & 2.59491500 \\
\hline $\mathrm{H}$ & -3.29712500 & 4.18938200 & 2.38270500 \\
\hline $\mathrm{C}$ & -1.95332400 & 2.18350300 & 1.19349200 \\
\hline $\mathrm{H}$ & -1.32659900 & 1.77037700 & 0.39985800 \\
\hline $\mathrm{H}$ & -1.33975500 & 2.91229800 & 1.73672000 \\
\hline $\mathrm{H}$ & -2.21368600 & 1.37582200 & 1.88735000 \\
\hline $\mathrm{C}$ & -2.82652700 & 4.03466300 & -0.29387700 \\
\hline $\mathrm{H}$ & -2.24344600 & 3.67531100 & -1.14604000 \\
\hline $\mathrm{H}$ & -3.71303100 & 4.55290800 & -0.67817000 \\
\hline $\mathrm{H}$ & -2.20491900 & 4.76044500 & 0.24204300 \\
\hline $\mathrm{C}$ & 6.82727400 & -0.78174400 & 0.46482400 \\
\hline $\mathrm{H}$ & 7.61479700 & -1.03210000 & -0.25714400 \\
\hline $\mathrm{H}$ & 6.68809300 & 0.30147600 & 0.44034400 \\
\hline $\mathrm{H}$ & 7.20685100 & -1.05351800 & 1.45629100 \\
\hline $\mathrm{C}$ & 6.31533400 & -0.04904200 & -2.63371500 \\
\hline $\mathrm{H}$ & 6.34028200 & 0.18053500 & -3.70512900 \\
\hline
\end{tabular}




\begin{tabular}{|c|c|c|c|}
\hline $\mathrm{H}$ & 7.34455000 & 0.04347800 & -2.26466600 \\
\hline $\mathrm{H}$ & 6.01340300 & -1.09237000 & -2.51823300 \\
\hline $\mathrm{C}$ & 1.85128300 & -3.78166700 & -0.24006100 \\
\hline $\mathrm{C}$ & 1.38576200 & -4.18700800 & -1.50962700 \\
\hline $\mathrm{C}$ & 1.11609900 & -4.11919100 & 0.91549300 \\
\hline $\mathrm{C}$ & 0.19044200 & -4.90443000 & -1.60179900 \\
\hline $\mathrm{C}$ & -0.08043700 & -4.83484800 & 0.77589200 \\
\hline $\mathrm{C}$ & -0.56760800 & -5.22786400 & -0.47318200 \\
\hline $\mathrm{H}$ & -0.16515900 & -5.20788600 & -2.58459500 \\
\hline $\mathrm{H}$ & -0.64396100 & -5.09410000 & 1.67117500 \\
\hline $\mathrm{C}$ & 2.61949900 & 3.78824900 & -0.20792100 \\
\hline $\mathrm{C}$ & 2.80653000 & 4.21771100 & 1.12361900 \\
\hline $\mathrm{C}$ & 1.60292300 & 4.36850500 & -0.99506400 \\
\hline $\mathrm{C}$ & 1.99134500 & 5.23262000 & 1.63500100 \\
\hline $\mathrm{C}$ & 0.81556900 & 5.38744800 & -0.44558200 \\
\hline $\mathrm{C}$ & 0.99739900 & 5.84125600 & 0.86347100 \\
\hline $\mathrm{H}$ & 2.14582500 & 5.56227400 & 2.66113600 \\
\hline $\mathrm{H}$ & 0.03897900 & 5.83782900 & -1.06141200 \\
\hline $\mathrm{C}$ & -1.88649100 & -5.95119300 & -0.61087200 \\
\hline $\mathrm{H}$ & -1.81902600 & -6.78201300 & -1.32333900 \\
\hline $\mathrm{H}$ & -2.22572300 & -6.35643500 & 0.34882800 \\
\hline $\mathrm{H}$ & -2.66246200 & -5.26877700 & -0.98089000 \\
\hline $\mathrm{C}$ & 2.15244400 & -3.85080400 & -2.76876800 \\
\hline $\mathrm{H}$ & 2.11444400 & -2.77458000 & -2.97397300 \\
\hline $\mathrm{H}$ & 3.21029200 & -4.12608000 & -2.68628600 \\
\hline $\mathrm{H}$ & 1.73120900 & -4.37560100 & -3.63247300 \\
\hline $\mathrm{C}$ & 3.86973400 & 3.60009000 & 2.00368400 \\
\hline $\mathrm{H}$ & 3.64256800 & 2.54860900 & 2.21464900 \\
\hline $\mathrm{H}$ & 4.85623200 & 3.62655900 & 1.52619500 \\
\hline $\mathrm{H}$ & 3.94142400 & 4.13011700 & 2.95906700 \\
\hline $\mathrm{C}$ & 1.32166800 & 3.87914500 & -2.39852900 \\
\hline $\mathrm{H}$ & 0.49472800 & 4.44200800 & -2.84532400 \\
\hline
\end{tabular}




$\begin{array}{llll}\mathrm{H} & 2.19122100 & 3.98320400 & -3.05712600 \\ \mathrm{H} & 1.05103200 & 2.81694400 & -2.37889900 \\ \mathrm{C} & 0.16887300 & 6.97744100 & 1.41962800 \\ \mathrm{H} & -0.00888500 & 6.85755800 & 2.49447600 \\ \mathrm{H} & 0.67256300 & 7.94426600 & 1.28291500 \\ \mathrm{H} & -0.80434600 & 7.04865200 & 0.92086300 \\ \mathrm{C} & 1.57471700 & -3.69660300 & 2.29440300 \\ \mathrm{H} & 1.66929800 & -2.60656300 & 2.34754000 \\ \mathrm{H} & 0.85416200 & -4.02096800 & 3.05370700 \\ \mathrm{H} & 2.54868300 & -4.12403200 & 2.55808700 \\ \mathrm{C} & -2.20832500 & -1.88892400 & 4.30054000 \\ \mathrm{O} & -1.58765800 & -0.92788600 & 3.42142800 \\ \mathrm{C} & -0.48074100 & -0.27923800 & 4.09490600 \\ \mathrm{C} & -0.11600700 & -1.21340900 & 5.24651100 \\ \mathrm{C} & -1.49351100 & -1.76196600 & 5.65371900 \\ \mathrm{H} & -3.28168600 & -1.67411000 & 4.35921200 \\ \mathrm{H} & -2.07970600 & -2.88911300 & 3.86745000 \\ \mathrm{H} & 0.30657900 & -0.13303600 & 3.35411900 \\ \mathrm{H} & -0.81834300 & 0.69843600 & 4.46877900 \\ \mathrm{H} & 0.53109700 & -2.02169300 & 4.88728000 \\ \mathrm{H} & 0.40053800 & -0.69672200 & 6.06127200 \\ \mathrm{H} & -1.44326000 & -2.71557400 & 6.18818900 \\ & -2.01341100 & -1.04196700 & 6.29690600\end{array}$

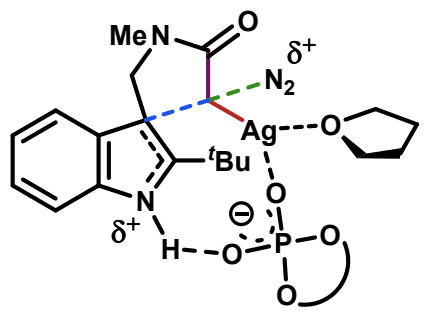

$\mathrm{TS} 1_{\text {carbenoid }}$

Zero-point correction $=$ 1.023188 (Hartree/Particle)

Thermal correction to Energy= 1.090299 
$\begin{array}{lc}\text { Thermal correction to Enthalpy }= & 1.091243 \\ \text { Thermal correction to Gibbs Free Energy= } & 0.914413 \\ \text { Sum of electronic and zero-point Energies= } & -3174.202056 \\ \text { Sum of electronic and thermal Energies= } & -3174.134945 \\ \text { Sum of electronic and thermal Enthalpies= } & -3174.134001 \\ \text { Sum of electronic and thermal Free Energies }= & -3174.310831\end{array}$

Cartesian Coordinates

$\begin{array}{llll}\text { Atom } & \mathrm{X} & \mathrm{Y} & \mathrm{Z}\end{array}$

C

C

C

C

C

$\mathrm{C}$

$\mathrm{H}$

C

C

C

C

C

C

$\mathrm{H}$

$\mathrm{O}$

$\mathrm{O}$

$\mathrm{P}$

Ag

$\mathrm{O}$

O

$\mathrm{H}$

$\mathrm{H}$ $\begin{array}{lll}4.44021500 & 1.26234500 & -0.86676800\end{array}$

$\begin{array}{llll}5.60336400 & 1.11927600 & -1.63593900\end{array}$

$\begin{array}{lll}6.12594900 & -0.13694300 & -1.91986800\end{array}$

$5.55294000-1.30599300-1.40155400$

$\begin{array}{lll}4.42600600 & -1.19175500 & -0.55683800\end{array}$

$\begin{array}{lll}3.86394200 & 0.08826700 & -0.36657200\end{array}$

$\begin{array}{lll}6.08386400 & 2.01012200 & -2.03158500\end{array}$

$\begin{array}{lll}3.81281900 & -2.36789800 & 0.13518700\end{array}$

$2.43980700 \quad-2.63995400 \quad-0.04153100$

$\begin{array}{lll}4.55435100 & -3.20270900 & 1.00236900\end{array}$

$\begin{array}{lll}1.79938000 & -3.75060100 & 0.52447400\end{array}$

$\begin{array}{lll}3.92133800 & -4.31839400 & 1.56585700\end{array}$

$2.58347700 \quad-4.60019300 \quad 1.31661200$

$2.12156100-5.47846900 \quad 1.75975000$

$\begin{array}{lll}1.70121700 & -1.79107100 & -0.85539500\end{array}$

$\begin{array}{lll}2.70227800 & 0.19044000 & 0.39101100\end{array}$

$1.28939700 \quad-0.29333100 \quad-0.30387900$

$\begin{array}{lll}-1.80468100 & -0.08937600 & 0.87888300\end{array}$

$\begin{array}{lll}0.33550000 & -0.40490200 & 0.87771200\end{array}$

$0.92098100 \quad 0.51464300-1.51309900$

$\begin{array}{lll}6.99887100 & -0.22016700 & -2.56345800\end{array}$

$\begin{array}{lll}4.49148500 & -4.96924700 & 2.22488600\end{array}$ 


\begin{tabular}{llll}
$\mathrm{C}$ & -5.01187400 & -0.48585600 & 0.11119600 \\
$\mathrm{O}$ & -5.39018400 & -1.52318100 & 0.66165400 \\
$\mathrm{~N}$ & -5.54180200 & -0.04401300 & -1.07217800 \\
$\mathrm{C}$ & -6.72732500 & -0.73030200 & -1.57937600 \\
$\mathrm{H}$ & -7.64031400 & -0.16901600 & -1.33537400 \\
$\mathrm{H}$ & -6.66347500 & -0.83407400 & -2.66713300 \\
$\mathrm{H}$ & -6.78436500 & -1.71418700 & -1.11662200 \\
$\mathrm{C}$ & -5.22349300 & 1.26744100 & -1.67437300 \\
$\mathrm{H}$ & -5.57906300 & 1.21458500 & -2.70756000 \\
$\mathrm{H}$ & -5.82585000 & 2.04835300 & -1.18649500 \\
$\mathrm{C}$ & -3.77383600 & 1.64923000 & -1.59785800 \\
$\mathrm{C}$ & -2.65472600 & 1.05105200 & -2.20984300 \\
$\mathrm{C}$ & -3.29435700 & 2.83211100 & -0.91326800 \\
$\mathrm{~N}$ & -1.54064000 & 1.76769900 & -1.85211800 \\
$\mathrm{C}$ & -1.88573200 & 2.84496900 & -1.06132700 \\
$\mathrm{H}$ & -0.57864000 & 1.40247000 & -1.91131600 \\
$\mathrm{C}$ & -3.90720900 & 3.86030500 & -0.17372500 \\
$\mathrm{H}$ & -4.98786200 & 3.90090900 & -0.05516200 \\
$\mathrm{C}$ & -3.10780000 & 4.84866200 & 0.39374300 \\
$\mathrm{H}$ & -3.57246600 & 5.65435400 & 0.95596000 \\
$\mathrm{C}$ & -1.70539200 & 4.83057800 & 0.24335100 \\
$\mathrm{H}$ & -1.10892700 & 5.61721600 & 0.69702700 \\
$\mathrm{C}$ & -1.07296300 & 3.82722800 & -0.48527400 \\
$\mathrm{H}$ & 0.00567200 & 3.80904100 & -0.61226100 \\
$\mathrm{C}$ & -2.50018700 & -0.12993900 & -3.17222200 \\
$\mathrm{C}$ & -1.31367200 & 0.13286400 & -4.13351200 \\
$\mathrm{H}$ & -1.44407400 & 1.07272600 & -4.68208300 \\
$\mathrm{H}$ & -0.35588800 & 0.16250500 & -3.60735700 \\
$\mathrm{H}$ & -1.26075600 & -0.68158600 & -4.86486800 \\
& -2.22323100 & -1.44101700 & -2.39954600 \\
$\mathrm{H}$ & -3.03641300 & -1.68635200 & -1.70985500 \\
\hline & -2.12284100 & -2.27274200 & -3.10684000
\end{tabular}




\begin{tabular}{|c|c|c|c|}
\hline $\mathrm{H}$ & -1.29004600 & -1.38092800 & -1.83280300 \\
\hline $\mathrm{C}$ & -3.76529700 & -0.31764300 & -4.03462500 \\
\hline & -4.61518100 & -0.66374400 & -3.44234200 \\
\hline & -4.04450300 & 0.60702700 & -4.55327000 \\
\hline & -3.57079100 & -1.08116300 & -4.79592800 \\
\hline & 5.99051200 & -2.92286400 & 1.38990100 \\
\hline & 6.69496100 & -3.55120700 & 0.83068700 \\
\hline & 6.26872400 & -1.88063600 & 1.21707600 \\
\hline & 6.14136900 & -3.14359600 & 2.45242500 \\
\hline $\mathrm{C}$ & 6.14168700 & -2.63755100 & -1.81543900 \\
\hline $\mathrm{H}$ & 6.33848900 & -2.63994300 & -2.89364400 \\
\hline $\mathrm{H}$ & 7.09931700 & -2.83287600 & -1.31675600 \\
\hline $\mathrm{H}$ & 5.47294600 & -3.47179900 & -1.59275400 \\
\hline $\mathrm{C}$ & 0.34091900 & -4.02515800 & 0.32692200 \\
\hline $\mathrm{C}$ & -0.13360100 & -4.51989200 & -0.90373400 \\
\hline $\mathrm{C}$ & -0.56228800 & -3.81717600 & 1.39426400 \\
\hline $\mathrm{C}$ & -1.50011900 & -4.79345400 & -1.04877300 \\
\hline $\mathrm{C}$ & -1.91969200 & -4.09114900 & 1.20132900 \\
\hline $\mathrm{C}$ & -2.41302900 & -4.57991300 & -0.01419100 \\
\hline $\mathrm{H}$ & -1.85772100 & -5.18346500 & -2.00009800 \\
\hline $\mathrm{H}$ & -2.61218200 & -3.92225400 & 2.02438000 \\
\hline $\mathrm{C}$ & 3.86665000 & 2.61885500 & -0.59617200 \\
\hline $\mathrm{C}$ & 3.99919500 & 3.19753500 & 0.68365200 \\
\hline $\mathrm{C}$ & 3.22845300 & 3.33882500 & -1.62980100 \\
\hline $\mathrm{C}$ & 3.49735500 & 4.48478700 & 0.90726400 \\
\hline $\mathrm{C}$ & 2.74249000 & 4.62404700 & -1.36181800 \\
\hline $\mathrm{C}$ & 2.86970100 & 5.21880800 & -0.10240800 \\
\hline $\mathrm{H}$ & 3.60833600 & 4.92645600 & 1.89604700 \\
\hline $\mathrm{H}$ & 2.25161700 & 5.17469000 & -2.16255800 \\
\hline U & -3.88754000 & -4.86448400 & -0.18733100 \\
\hline I & -4.11253000 & -5.19170500 & -1.20806300 \\
\hline & -4.22448200 & -5.65513200 & 0.49587200 \\
\hline
\end{tabular}


Supporting Information

\begin{tabular}{|c|c|c|c|}
\hline $\mathrm{H}$ & -4.49102400 & -3.97414900 & 0.02774900 \\
\hline $\mathrm{C}$ & 0.80314600 & -4.75470600 & -2.06730200 \\
\hline H & 1.18801200 & -3.80593200 & -2.45957300 \\
\hline $\mathrm{H}$ & 1.67073400 & -5.35716400 & -1.77419900 \\
\hline $\mathrm{H}$ & 0.28987300 & -5.27434900 & -2.88292400 \\
\hline $\mathrm{C}$ & 4.67092100 & 2.45392000 & 1.81625500 \\
\hline $\mathrm{H}$ & 4.07074800 & 1.59341300 & 2.13441600 \\
\hline $\mathrm{H}$ & 5.65398100 & 2.06911700 & 1.52128100 \\
\hline & 4.80935200 & 3.10781800 & 2.68335600 \\
\hline $\mathrm{C}$ & 3.02925700 & 2.73860300 & -3.00403800 \\
\hline $\mathrm{H}$ & 2.48543100 & 3.43343700 & -3.65259600 \\
\hline $\mathrm{H}$ & 3.98092400 & 2.50163900 & -3.49317000 \\
\hline $\mathrm{H}$ & 2.45508900 & 1.80845200 & -2.93504600 \\
\hline $\mathrm{C}$ & 2.37260800 & 6.62474800 & 0.14711800 \\
\hline $\mathrm{H}$ & 2.16127600 & 6.79305000 & 1.20881100 \\
\hline $\mathrm{H}$ & 3.11774000 & 7.37228000 & -0.15773000 \\
\hline $\mathrm{H}$ & 1.45798000 & 6.83449100 & -0.41932300 \\
\hline $\mathrm{C}$ & -0.09242000 & -3.28501300 & 2.73083000 \\
\hline $\mathrm{H}$ & 0.45200400 & -2.34344800 & 2.60296300 \\
\hline $\mathrm{H}$ & -0.94569400 & -3.10556600 & 3.39414300 \\
\hline $\mathrm{H}$ & 0.58105800 & -3.98409600 & 3.24030400 \\
\hline $\mathrm{C}$ & -3.90715900 & 0.34449400 & 0.73582200 \\
\hline $\mathrm{H}$ & -4.15220300 & 1.38933300 & 0.91954300 \\
\hline $\mathrm{N}$ & -4.34143300 & -0.10003000 & 2.52040200 \\
\hline $\mathrm{N}$ & -4.29929900 & -0.35722200 & 3.59599100 \\
\hline $\mathrm{C}$ & -0.41614100 & 0.62131600 & 4.21869500 \\
\hline $\mathrm{O}$ & -1.52082200 & 1.13828100 & 3.46502700 \\
\hline $\mathrm{C}$ & -1.36970300 & 2.56629000 & 3.47224500 \\
\hline $\mathrm{C}$ & 0.15201700 & 2.84828800 & 3.47382600 \\
\hline $\mathrm{C}$ & 0.78615400 & 1.46637200 & 3.78295000 \\
\hline $\mathrm{H}$ & -0.62123900 & 0.73276600 & 5.29628800 \\
\hline & -0.32285400 & -0.44036600 & 3.9820370 \\
\hline
\end{tabular}


Supporting Information

$\begin{array}{llll}\mathrm{H} & -1.88856000 & 2.95830200 & 2.59455900 \\ \mathrm{H} & -1.84723400 & 2.97540600 & 4.37619900 \\ \mathrm{H} & 0.49032000 & 3.22778100 & 2.50594400 \\ \mathrm{H} & 0.40710300 & 3.59689700 & 4.23110700 \\ \mathrm{H} & 1.22343300 & 1.03525400 & 2.87940700 \\ \mathrm{H} & 1.55922700 & 1.51744200 & 4.55623700\end{array}$

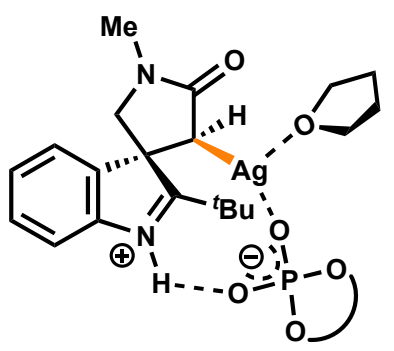

CP3

Zero-point correction $=$

1.023589 (Hartree/Particle)

Thermal correction to Energy=

1.091430

Thermal correction to Enthalpy=

1.092374

Thermal correction to Gibbs Free Energy=

0.911968

Sum of electronic and zero-point Energies=

$-3174.266500$

Sum of electronic and thermal Energies=

$-3174.198659$

Sum of electronic and thermal Enthalpies=

$-3174.197715$

Sum of electronic and thermal Free Energies $=\quad-3174.378121$

Cartesian Coordinates

$\begin{array}{lccc}\text { Atom } & \mathrm{X} & \mathrm{Y} & \mathrm{Z} \\ \mathrm{A} & 4.69660100 & 0.99708700 & -0.57706700 \\ \mathrm{C} & 5.87144100 & 0.78794100 & -1.31319900 \\ \mathrm{C} & 6.27875500 & -0.49243900 & -1.66771000 \\ \mathrm{C} & 5.56974700 & -1.62871000 & -1.25573000 \\ \mathrm{C} & 4.42586500 & -1.45696800 & -0.44474100 \\ \mathrm{C} & 3.98593300 & -0.14244800 & -0.17964000 \\ \mathrm{H} & 6.45480800 & 1.64975500 & -1.62632300\end{array}$




\begin{tabular}{|c|c|c|c|}
\hline $\mathrm{C}$ & 3.66865400 & -2.60784200 & 0.13745000 \\
\hline $\mathrm{C}$ & 2.28416700 & -2.72117700 & -0.10889800 \\
\hline $\mathrm{C}$ & 4.28319800 & -3.56859700 & 0.97266300 \\
\hline $\mathrm{C}$ & 1.50773200 & -3.78717700 & 0.36519500 \\
\hline $\mathrm{C}$ & 3.51404000 & -4.64181500 & 1.44061200 \\
\hline $\mathrm{C}$ & 2.16498400 & -4.76091800 & 1.12935200 \\
\hline $\mathrm{H}$ & 1.59335900 & -5.60553300 & 1.50452400 \\
\hline $\mathrm{O}$ & 1.67791800 & -1.74524800 & -0.89244700 \\
\hline $\mathrm{O}$ & 2.81234200 & 0.02491300 & 0.54604600 \\
\hline $\mathrm{P}$ & 1.38499100 & -0.26394000 & -0.22608800 \\
\hline $\mathrm{Ag}$ & -1.91420400 & -0.16545200 & 0.68352300 \\
\hline $\mathrm{O}$ & 0.35316700 & -0.35544400 & 0.87509000 \\
\hline $\mathrm{O}$ & 1.19742000 & 0.67598600 & -1.39388300 \\
\hline $\mathrm{H}$ & 7.16615700 & -0.62052000 & -2.28354700 \\
\hline $\mathrm{H}$ & 3.98439800 & -5.38810700 & 2.07686900 \\
\hline $\mathrm{C}$ & -4.90918900 & 0.20211100 & -0.61583200 \\
\hline $\mathrm{O}$ & -5.48340900 & -0.75654400 & -0.10983700 \\
\hline $\mathrm{N}$ & -5.40169000 & 0.87384400 & -1.73979700 \\
\hline $\mathrm{C}$ & -6.75264500 & 0.65045900 & -2.20946000 \\
\hline $\mathrm{H}$ & -7.44758900 & 1.41293400 & -1.82449100 \\
\hline $\mathrm{H}$ & -6.79628300 & 0.66535300 & -3.30527500 \\
\hline $\mathrm{H}$ & -7.06936200 & -0.32819600 & -1.84371200 \\
\hline $\mathrm{C}$ & -4.74184200 & 2.15180500 & -1.93844100 \\
\hline $\mathrm{H}$ & -4.69562400 & 2.42659400 & -2.99638500 \\
\hline $\mathrm{H}$ & -5.27611700 & 2.96239700 & -1.41358400 \\
\hline $\mathrm{C}$ & -3.36212200 & 1.94811100 & -1.28334700 \\
\hline $\mathrm{C}$ & -2.21709800 & 1.34764800 & -2.11788000 \\
\hline $\mathrm{C}$ & -2.68633000 & 3.14076000 & -0.65385200 \\
\hline $\mathrm{N}$ & -1.10165500 & 1.95206400 & -1.77320400 \\
\hline $\mathrm{C}$ & -1.31523100 & 3.03278100 & -0.88687200 \\
\hline $\mathrm{H}$ & -0.14398900 & 1.52107600 & -1.85840800 \\
\hline $\mathrm{C}$ & -3.15640100 & 4.16525700 & 0.16040400 \\
\hline
\end{tabular}




\begin{tabular}{|c|c|c|c|}
\hline $\mathrm{H}$ & -4.21638400 & 4.27691100 & 0.37179500 \\
\hline $\mathrm{C}$ & -2.22692700 & 5.05818500 & 0.71149100 \\
\hline $\mathrm{H}$ & -2.57872600 & 5.87440200 & 1.33624200 \\
\hline $\mathrm{C}$ & -0.85297800 & 4.91335700 & 0.47697400 \\
\hline $\mathrm{H}$ & -0.15286700 & 5.60963800 & 0.92865800 \\
\hline $\mathrm{C}$ & -0.36601500 & 3.88279800 & -0.33342700 \\
\hline $\mathrm{H}$ & 0.69582700 & 3.75126900 & -0.51691400 \\
\hline $\mathrm{C}$ & -2.20119200 & 0.26847300 & -3.20343300 \\
\hline $\mathrm{C}$ & -0.82344600 & 0.23309500 & -3.90702900 \\
\hline $\mathrm{H}$ & -0.57480100 & 1.20030100 & -4.35999400 \\
\hline $\mathrm{H}$ & -0.01330900 & -0.05334600 & -3.23207900 \\
\hline $\mathrm{H}$ & -0.86728000 & -0.50850200 & -4.71097200 \\
\hline $\mathrm{C}$ & -2.48951000 & -1.14344500 & -2.63828000 \\
\hline $\mathrm{H}$ & -3.46259000 & -1.21292700 & -2.15163300 \\
\hline $\mathrm{H}$ & -2.47462600 & -1.85185500 & -3.47453000 \\
\hline $\mathrm{H}$ & -1.72335500 & -1.45363700 & -1.92395600 \\
\hline $\mathrm{C}$ & -3.26695600 & 0.59919900 & -4.28166000 \\
\hline $\mathrm{H}$ & -4.28130700 & 0.46861300 & -3.90161900 \\
\hline $\mathrm{H}$ & -3.15167500 & 1.61558200 & -4.67638300 \\
\hline $\mathrm{H}$ & -3.13012800 & -0.09448300 & -5.11781600 \\
\hline $\mathrm{C}$ & 5.72337300 & -3.46785500 & 1.42751800 \\
\hline $\mathrm{H}$ & 6.38130700 & -4.13132900 & 0.85232900 \\
\hline $\mathrm{H}$ & 6.11553600 & -2.45240300 & 1.33455700 \\
\hline $\mathrm{H}$ & 5.80639000 & -3.76957800 & 2.47749900 \\
\hline $\mathrm{C}$ & 6.03864400 & -2.98286900 & -1.74271800 \\
\hline $\mathrm{H}$ & 6.28641900 & -2.93141900 & -2.80914300 \\
\hline $\mathrm{H}$ & 6.94576200 & -3.31238700 & -1.22079100 \\
\hline $\mathrm{H}$ & 5.27792100 & -3.75513400 & -1.60953700 \\
\hline C & 0.03588000 & -3.89007600 & 0.11225000 \\
\hline $\mathrm{C}$ & -0.44648900 & -4.28880000 & -1.14956500 \\
\hline $\mathrm{C}$ & -0.87439400 & -3.62499700 & 1.16134700 \\
\hline & -1.82870500 & -4.41072400 & -1.34521300 \\
\hline
\end{tabular}




\begin{tabular}{|c|c|c|c|}
\hline $\mathrm{C}$ & -2.24737400 & -3.73486100 & 0.91408200 \\
\hline $\mathrm{C}$ & -2.74867800 & -4.12789000 & -0.33330700 \\
\hline $\mathrm{H}$ & -2.19339000 & -4.73168200 & -2.31956300 \\
\hline $\mathrm{H}$ & -2.94483600 & -3.51910400 & 1.72098300 \\
\hline $\mathrm{C}$ & 4.25495100 & 2.38739800 & -0.23931600 \\
\hline $\mathrm{C}$ & 4.37459400 & 2.86670500 & 1.08292300 \\
\hline $\mathrm{C}$ & 3.76704100 & 3.24089400 & -1.25279500 \\
\hline $\mathrm{C}$ & 4.01556600 & 4.18892000 & 1.36606300 \\
\hline $\mathrm{C}$ & 3.41984200 & 4.55751900 & -0.92427500 \\
\hline $\mathrm{C}$ & 3.54284900 & 5.05506300 & 0.37675000 \\
\hline $\mathrm{H}$ & 4.11566000 & 4.55162600 & 2.38759300 \\
\hline $\mathrm{H}$ & 3.04929100 & 5.21275000 & -1.71117600 \\
\hline $\mathrm{C}$ & -4.23875100 & -4.22883100 & -0.56553300 \\
\hline $\mathrm{H}$ & -4.46052300 & -4.60105800 & -1.57167200 \\
\hline $\mathrm{H}$ & -4.70855200 & -4.91143600 & 0.15388000 \\
\hline $\mathrm{H}$ & -4.72406700 & -3.25208900 & -0.44667500 \\
\hline $\mathrm{C}$ & 0.50077100 & -4.58569200 & -2.29068500 \\
\hline $\mathrm{H}$ & 1.01872700 & -3.67720600 & -2.62080600 \\
\hline $\mathrm{H}$ & 1.27372000 & -5.30646800 & -1.99921200 \\
\hline $\mathrm{H}$ & -0.03935400 & -4.99784000 & -3.14935800 \\
\hline $\mathrm{C}$ & 4.88289700 & 1.97924700 & 2.19651800 \\
\hline $\mathrm{H}$ & 4.16836700 & 1.17796500 & 2.41756600 \\
\hline $\mathrm{H}$ & 5.83211600 & 1.50018300 & 1.93020700 \\
\hline $\mathrm{H}$ & 5.03969400 & 2.55597800 & 3.11372300 \\
\hline $\mathrm{C}$ & 3.58143100 & 2.75482600 & -2.67371700 \\
\hline $\mathrm{H}$ & 3.13166500 & 3.53849000 & -3.29296900 \\
\hline $\mathrm{H}$ & 4.53090600 & 2.46843100 & -3.14004900 \\
\hline $\mathrm{H}$ & 2.92824500 & 1.87601300 & -2.69525900 \\
\hline $\mathrm{C}$ & 3.20927600 & 6.49487700 & 0.69636300 \\
\hline $\mathrm{H}$ & 2.83892300 & 6.60413600 & 1.72205400 \\
\hline $\mathrm{H}$ & 4.09374600 & 7.13994600 & 0.60397000 \\
\hline $\mathrm{H}$ & 2.44918100 & 6.89335900 & 0.01477900 \\
\hline
\end{tabular}


Supporting Information

$\begin{array}{lrrr}\mathrm{C} & -0.39743600 & -3.23183800 & 2.54275100 \\ \mathrm{H} & 0.37378400 & -2.45903900 & 2.48883500 \\ \mathrm{H} & -1.22599500 & -2.83892500 & 3.13956700 \\ \mathrm{H} & 0.02984500 & -4.08787300 & 3.08085100 \\ \mathrm{C} & -3.65927600 & 0.87849700 & -0.15027800 \\ \mathrm{H} & -3.91956200 & 1.43235100 & 0.76061000 \\ \mathrm{~N} & -5.58643300 & -1.17230100 & 3.07412700 \\ \mathrm{~N} & -5.27724600 & -2.22940900 & 2.98905100 \\ \mathrm{C} & -0.47198300 & 0.46427200 & 3.90495700 \\ \mathrm{O} & -1.75875500 & 0.07470300 & 3.40262100 \\ \mathrm{C} & -2.70327700 & 0.92404700 & 4.05847600 \\ \mathrm{C} & -2.03417500 & 2.31339800 & 4.14498600 \\ \mathrm{C} & -0.52086700 & 2.00070000 & 3.97709400 \\ \mathrm{H} & -0.32530100 & 0.02445400 & 4.90439700 \\ \mathrm{H} & 0.27579700 & 0.07468300 & 3.21495800 \\ \mathrm{H} & -3.62706000 & 0.90461600 & 3.47583100 \\ \mathrm{H} & -2.91574200 & 0.52786400 & 5.06412700 \\ \mathrm{H} & -2.38486600 & 2.96904900 & 3.34219500 \\ \mathrm{H} & -2.25936900 & 2.80490900 & 5.09698300 \\ \mathrm{H} & -0.13963100 & 2.43256400 & 3.04711300 \\ \mathrm{H} & 0.08549600 & 2.39015400 & 4.80100400\end{array}$

\section{$\mathrm{PhCO}_{2} \mathrm{H}$}

Zero-point correction $=$

0.115915 (Hartree/Particle)

Thermal correction to Energy=

0.123010

Thermal correction to Enthalpy=

0.123954

Thermal correction to Gibbs Free Energy=

0.083904

Sum of electronic and zero-point Energies= $-420.706217$

Sum of electronic and thermal Energies= $-420.699122$

Sum of electronic and thermal Enthalpies= $-420.698178$

Sum of electronic and thermal Free Energies= $-420.738228$ 
Cartesian Coordinates

$\begin{array}{llll}\text { Atom } & \mathrm{X} & \mathrm{Y} & \mathrm{Z}\end{array}$

$\begin{array}{lrrr}\mathrm{C} & -1.90401400 & 1.18697600 & -0.00003700 \\ \mathrm{C} & -0.51229600 & 1.22239900 & -0.00002500 \\ \mathrm{C} & 0.22020400 & 0.02738500 & 0.00000700 \\ \mathrm{C} & -0.45030800 & -1.20384700 & 0.00004200 \\ \mathrm{C} & -1.84377000 & -1.23431500 & 0.00003300 \\ \mathrm{C} & -2.57080000 & -0.04140200 & -0.00000900 \\ \mathrm{H} & -2.46976700 & 2.11443800 & -0.00006800 \\ \mathrm{H} & 0.02806800 & 2.16332000 & -0.00004200 \\ \mathrm{H} & 0.12067000 & -2.12569100 & 0.00008300 \\ \mathrm{H} & -2.36309100 & -2.18859800 & 0.00005900 \\ \mathrm{H} & -3.65728500 & -0.06886400 & -0.00001800 \\ \mathrm{C} & 1.70347400 & 0.12162900 & 0.00000500 \\ \mathrm{O} & 2.33671800 & 1.15877900 & 0.00007900 \\ \mathrm{O} & 2.31516200 & -1.09198600 & -0.00008300 \\ \mathrm{H} & 3.27142100 & -0.90190100 & -0.00007800\end{array}$

$\mathrm{N}_{2}$

Zero-point correction $=\quad 0.005598($ Hartree/Particle $)$

Thermal correction to Energy $=\quad 0.007959$

Thermal correction to Enthalpy $=0.008903$

Thermal correction to Gibbs Free Energy= $\quad-0.012851$

Sum of electronic and zero-point Energies $=\quad-109.518530$

Sum of electronic and thermal Energies $=\quad-109.516170$

Sum of electronic and thermal Enthalpies = $\quad-109.515226$

Sum of electronic and thermal Free Energies= $\quad-109.536980$

Cartesian Coordinates

$\begin{array}{llll}\text { Atom } & \mathrm{X} & \mathrm{Y} & \mathrm{Z}\end{array}$ 


$\begin{array}{llll}\mathrm{N} & 0.00000000 & 0.00000000 & 0.55274900 \\ \mathrm{~N} & 0.00000000 & 0.00000000 & -0.55274900\end{array}$

THF

Zero-point correction= 0.117360 (Hartree/Particle)

Thermal correction to Energy= 0.122315

Thermal correction to Enthalpy= 0.123259

Thermal correction to Gibbs Free Energy= 0.088709

Sum of electronic and zero-point Energies $=$ $-232.332089$

Sum of electronic and thermal Energies= $-232.327135$

Sum of electronic and thermal Enthalpies= $-232.326191$

Sum of electronic and thermal Free Energies= $-232.360740$

Cartesian Coordinates

$\begin{array}{lccc}\text { Atom } & \mathrm{X} & \mathrm{Y} & \mathrm{Z} \\ \mathrm{-} & & & \\ \mathrm{C} & 1.16544900 & -0.43058700 & 0.13177800 \\ \mathrm{O} & 0.00009200 & -1.25151500 & 0.00021000 \\ \mathrm{C} & -1.16530500 & -0.43078800 & -0.13204500 \\ \mathrm{C} & -0.73393800 & 0.99661100 & 0.22696400 \\ \mathrm{C} & 0.73369200 & 0.99682100 & -0.22684400 \\ \mathrm{H} & 1.53575500 & -0.48315100 & 1.16750700 \\ \mathrm{H} & 1.94875200 & -0.82335700 & -0.52723300 \\ \mathrm{H} & -1.53501800 & -0.48324800 & -1.16800500 \\ \mathrm{H} & -1.94890300 & -0.82379800 & 0.52646100 \\ \mathrm{H} & -1.34429400 & 1.76116000 & -0.26375300 \\ \mathrm{H} & -0.79716400 & 1.15512500 & 1.31030900 \\ \mathrm{H} & 0.79685500 & 1.15567100 & -1.31014300 \\ \mathrm{H} & 1.34389500 & 1.76137400 & 0.26405600\end{array}$




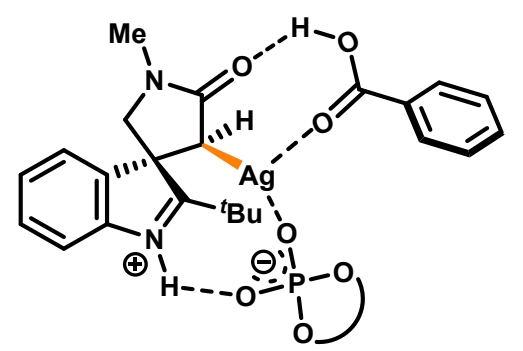

CP4

Zero-point correction= 1.014584 (Hartree/Particle)

Thermal correction to Energy= 1.080710

Thermal correction to Enthalpy= 1.081654

Thermal correction to Gibbs Free Energy= 0.906820

Sum of electronic and zero-point Energies= $-3253.136308$

Sum of electronic and thermal Energies= $-3253.070182$

Sum of electronic and thermal Enthalpies= $-3253.069238$

Sum of electronic and thermal Free Energies= $-3253.244071$

Cartesian Coordinates

Atom $\mathrm{X}$ Y Z

$\begin{array}{llrl}\mathrm{C} & -4.80635700 & 1.08866400 & -0.57763900 \\ \mathrm{C} & -6.15878900 & 0.99424900 & -0.21849800 \\ \mathrm{C} & -6.69647700 & -0.19347400 & 0.26034300 \\ \mathrm{C} & -5.92766800 & -1.36103000 & 0.34748700 \\ \mathrm{C} & -4.58162600 & -1.31953700 & -0.08071800 \\ \mathrm{C} & -4.03810300 & -0.07799300 & -0.47266200 \\ \mathrm{H} & -6.78432900 & 1.87903600 & -0.30163500 \\ \mathrm{C} & -3.72189400 & -2.54178700 & -0.10751900 \\ \mathrm{C} & -2.51374800 & -2.55153100 & 0.62038300 \\ \mathrm{C} & -4.07523400 & -3.69590400 & -0.84193200 \\ \mathrm{C} & -1.71980900 & -3.69719900 & 0.75998700 \\ \mathrm{C} & -3.27126200 & -4.83706800 & -0.72643800 \\ \mathrm{C} & -2.13948200 & -4.84876500 & 0.07927900 \\ \mathrm{H} & -1.54800600 & -5.75564300 & 0.17206200\end{array}$


Supporting Information

\begin{tabular}{|c|c|c|c|}
\hline $\mathrm{O}$ & -2.12927500 & -1.38366400 & 1.26716100 \\
\hline $\mathrm{O}$ & -2.69528300 & -0.02920800 & -0.82358400 \\
\hline $\mathrm{P}$ & -1.55176500 & -0.12858600 & 0.35851500 \\
\hline $\mathrm{Ag}$ & 1.86959900 & 0.15656000 & -0.24431900 \\
\hline $\mathrm{O}$ & -0.27791400 & -0.53142000 & -0.34977400 \\
\hline $\mathrm{O}$ & -1.59559400 & 1.07808700 & 1.26776000 \\
\hline $\mathrm{H}$ & -7.73603800 & -0.22149500 & 0.57899900 \\
\hline $\mathrm{H}$ & -3.54160500 & -5.73099100 & -1.28404200 \\
\hline $\mathrm{C}$ & 4.71790500 & 1.40413500 & 0.58321600 \\
\hline $\mathrm{O}$ & 5.46707700 & 0.69353300 & -0.10879100 \\
\hline $\mathrm{N}$ & 5.16282300 & 2.03834600 & 1.72086100 \\
\hline $\mathrm{C}$ & 6.53874800 & 1.99919100 & 2.16203100 \\
\hline $\mathrm{H}$ & 7.02991400 & 2.97313300 & 2.02250100 \\
\hline $\mathrm{H}$ & 6.60577100 & 1.72771800 & 3.22365700 \\
\hline $\mathrm{H}$ & 7.05801500 & 1.25021300 & 1.56169900 \\
\hline $\mathrm{C}$ & 4.21606100 & 2.99856200 & 2.25765400 \\
\hline $\mathrm{H}$ & 4.12984100 & 2.90283000 & 3.34565000 \\
\hline $\mathrm{H}$ & 4.54618100 & 4.02917900 & 2.05325600 \\
\hline $\mathrm{C}$ & 2.89063000 & 2.70222100 & 1.51282100 \\
\hline $\mathrm{C}$ & 1.78367500 & 1.91132700 & 2.22252100 \\
\hline $\mathrm{C}$ & 2.11406400 & 3.87153200 & 0.95287700 \\
\hline $\mathrm{N}$ & 0.61726900 & 2.40469200 & 1.85902600 \\
\hline $\mathrm{C}$ & 0.75504100 & 3.58304100 & 1.08361700 \\
\hline $\mathrm{H}$ & -0.29584700 & 1.86667300 & 1.83810400 \\
\hline $\mathrm{C}$ & 2.50820100 & 5.00772500 & 0.25858300 \\
\hline $\mathrm{H}$ & 3.55881300 & 5.25221400 & 0.12535800 \\
\hline $\mathrm{C}$ & 1.51359200 & 5.83645700 & -0.28067900 \\
\hline $\mathrm{H}$ & 1.80219300 & 6.73738700 & -0.81463500 \\
\hline $\mathrm{C}$ & 0.15801500 & 5.51144300 & -0.15818600 \\
\hline $\mathrm{H}$ & -0.59340200 & 6.15415500 & -0.60599100 \\
\hline $\mathrm{C}$ & -0.24979900 & 4.36182200 & 0.52795700 \\
\hline $\mathrm{H}$ & -1.29514400 & 4.08346000 & 0.60321300 \\
\hline
\end{tabular}




\begin{tabular}{|c|c|c|c|}
\hline $\mathrm{C}$ & 1.87280400 & 0.83784400 & 3.30092200 \\
\hline $\mathrm{C}$ & 0.66723700 & -0.12427800 & 3.27557600 \\
\hline $\mathrm{H}$ & -0.29496000 & 0.38583600 & 3.35874600 \\
\hline $\mathrm{H}$ & 0.65588500 & -0.72765800 & 2.36453400 \\
\hline $\mathrm{H}$ & 0.75503600 & -0.81352300 & 4.12080100 \\
\hline $\mathrm{C}$ & 3.16014400 & -0.00706800 & 3.25387200 \\
\hline $\mathrm{H}$ & 4.06982500 & 0.58643200 & 3.35569100 \\
\hline $\mathrm{H}$ & 3.12967100 & -0.72185800 & 4.08245900 \\
\hline $\mathrm{H}$ & 3.22585300 & -0.57765800 & 2.32347900 \\
\hline $\mathrm{C}$ & 1.82669500 & 1.62891900 & 4.64648500 \\
\hline $\mathrm{H}$ & 2.65870000 & 2.33227700 & 4.75463700 \\
\hline $\mathrm{H}$ & 0.88957800 & 2.18562000 & 4.74569100 \\
\hline $\mathrm{H}$ & 1.88815500 & 0.91070200 & 5.47120400 \\
\hline $\mathrm{C}$ & -5.25722500 & -3.74374700 & -1.78633400 \\
\hline $\mathrm{H}$ & -6.09225500 & -4.31880200 & -1.36669900 \\
\hline $\mathrm{H}$ & -5.63002100 & -2.74723300 & -2.03303100 \\
\hline $\mathrm{H}$ & -4.96870500 & -4.23858500 & -2.72068400 \\
\hline $\mathrm{C}$ & -6.56836600 & -2.59885400 & 0.93762500 \\
\hline $\mathrm{H}$ & -7.19753000 & -2.32320800 & 1.79139300 \\
\hline $\mathrm{H}$ & -7.21813700 & -3.10722800 & 0.21425300 \\
\hline $\mathrm{H}$ & -5.82684100 & -3.32306500 & 1.28227300 \\
\hline $\mathrm{C}$ & -0.47962800 & -3.73932900 & 1.59732000 \\
\hline $\mathrm{C}$ & -0.57775200 & -3.78085300 & 3.00388500 \\
\hline $\mathrm{C}$ & 0.78712000 & -3.81153400 & 0.97724900 \\
\hline $\mathrm{C}$ & 0.58993000 & -3.90824800 & 3.76551700 \\
\hline $\mathrm{C}$ & 1.92952900 & -3.92465300 & 1.77857600 \\
\hline $\mathrm{C}$ & 1.85346100 & -3.98695800 & 3.17351100 \\
\hline $\mathrm{H}$ & 0.50604600 & -3.95462400 & 4.85022000 \\
\hline $\mathrm{H}$ & 2.90339300 & -3.97451200 & 1.29454600 \\
\hline $\mathrm{C}$ & -4.26589600 & 2.39698500 & -1.06440400 \\
\hline $\mathrm{C}$ & -3.97745300 & 2.57862600 & -2.43527000 \\
\hline $\mathrm{C}$ & -4.13366500 & 3.48027600 & -0.17081400 \\
\hline
\end{tabular}




\begin{tabular}{|c|c|c|c|}
\hline $\mathrm{C}$ & -3.58424400 & 3.84114900 & -2.88814800 \\
\hline $\mathrm{C}$ & -3.74490800 & 4.73089300 & -0.67061600 \\
\hline $\mathrm{C}$ & -3.47022900 & 4.93511000 & -2.02489000 \\
\hline $\mathrm{H}$ & -3.37256000 & 3.97460000 & -3.94768100 \\
\hline $\mathrm{H}$ & -3.66417300 & 5.56852300 & 0.02077000 \\
\hline $\mathrm{C}$ & 3.09930100 & -4.16691400 & 4.01136800 \\
\hline $\mathrm{H}$ & 2.94030600 & -3.83859300 & 5.04456700 \\
\hline $\mathrm{H}$ & 3.40556900 & -5.22118000 & 4.05002900 \\
\hline $\mathrm{H}$ & 3.94528100 & -3.60324000 & 3.60141400 \\
\hline $\mathrm{C}$ & -1.91813300 & -3.69446000 & 3.69884900 \\
\hline $\mathrm{H}$ & -2.38043000 & -2.71249100 & 3.54412400 \\
\hline $\mathrm{H}$ & -2.62106800 & -4.44374100 & 3.31625800 \\
\hline $\mathrm{H}$ & -1.80975200 & -3.85177400 & 4.77697900 \\
\hline $\mathrm{C}$ & -4.09648700 & 1.43718200 & -3.41938600 \\
\hline $\mathrm{H}$ & -3.34371000 & 0.66632300 & -3.21859400 \\
\hline $\mathrm{H}$ & -5.07833500 & 0.95308700 & -3.35998400 \\
\hline $\mathrm{H}$ & -3.95405200 & 1.79091600 & -4.44546100 \\
\hline $\mathrm{C}$ & -4.37050900 & 3.31171500 & 1.31495400 \\
\hline $\mathrm{H}$ & -4.15854500 & 4.24505500 & 1.84790500 \\
\hline $\mathrm{H}$ & -5.40381700 & 3.02735400 & 1.54278300 \\
\hline $\mathrm{H}$ & -3.72171200 & 2.52629300 & 1.71931900 \\
\hline $\mathrm{C}$ & -3.06011400 & 6.29279500 & -2.54918200 \\
\hline $\mathrm{H}$ & -3.11899600 & 7.05843400 & -1.76789600 \\
\hline $\mathrm{H}$ & -2.03093500 & 6.28245000 & -2.93185000 \\
\hline $\mathrm{H}$ & -3.70314400 & 6.61430100 & -3.37778900 \\
\hline $\mathrm{C}$ & 0.93107300 & -3.76568200 & -0.52786700 \\
\hline $\mathrm{H}$ & 0.33889700 & -2.95005300 & -0.95321600 \\
\hline $\mathrm{H}$ & 1.97364900 & -3.60455200 & -0.81526100 \\
\hline $\mathrm{H}$ & 0.59090900 & -4.69952900 & -0.99383500 \\
\hline $\mathrm{C}$ & 3.30721200 & 1.74867100 & 0.32770100 \\
\hline $\mathrm{H}$ & 3.22658800 & 2.27362300 & -0.62840200 \\
\hline $\mathrm{C}$ & 2.77626200 & -3.13611100 & -5.69102700 \\
\hline
\end{tabular}




$\begin{array}{llrr}\text { C } & 2.82896600 & -2.54780100 & -4.42928300 \\ \mathrm{C} & 3.95077500 & -1.79777100 & -4.04992500 \\ \mathrm{C} & 5.02007200 & -1.64265900 & -4.94378200 \\ \mathrm{C} & 4.96460100 & -2.23501000 & -6.20456300 \\ \mathrm{C} & 3.84408900 & -2.98113600 & -6.57956300 \\ \mathrm{H} & 1.90404000 & -3.71480300 & -5.98247200 \\ \mathrm{H} & 2.01135400 & -2.65621200 & -3.72436200 \\ \mathrm{H} & 5.88434400 & -1.06138200 & -4.64242900 \\ \mathrm{H} & 5.79499000 & -2.11506700 & -6.89515200 \\ \mathrm{H} & 3.80292600 & -3.44111700 & -7.56359200 \\ \mathrm{C} & 3.97231600 & -1.18300000 & -2.69001600 \\ \mathrm{O} & 3.06811200 & -1.36987200 & -1.87467700 \\ \mathrm{O} & 5.03964100 & -0.43340100 & -2.46151700 \\ \mathrm{H} & 5.04690800 & -0.05234600 & -1.52741900\end{array}$

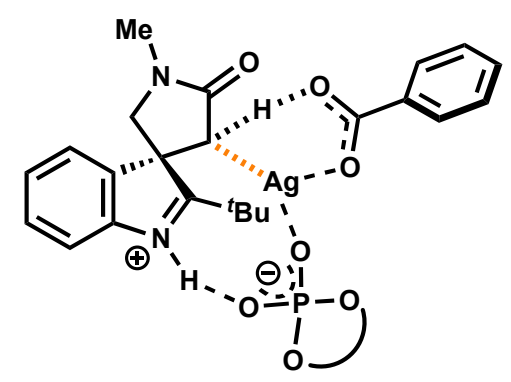

TS3 $_{\text {deargentation }}$

Zero-point correction $=$ 1.012891 (Hartree/Particle)

Thermal correction to Energy= 1.078179

Thermal correction to Enthalpy= 1.079123

Thermal correction to Gibbs Free Energy= 0.907390

Sum of electronic and zero-point Energies= $-3253.105476$

Sum of electronic and thermal Energies= $-3253.040188$

Sum of electronic and thermal Enthalpies= $-3253.039244$

Sum of electronic and thermal Free Energies= $-3253.210977$

Cartesian Coordinates

Atom $\mathrm{X}$ $\mathrm{Y}$ Z 
C

$-5.28999700$

0.16483200

0.13154100

C

$-6.48843100-0.28025600$

0.70770500

C

$-6.68793100-1.62108800$

1.01006400

C

$-5.72646500 \quad-2.59159700$

0.70148400

C

$-4.53889900-2.18655700$

0.05024800

C

$-4.32684200$

$-0.80829500$

$-0.16334700$

$\mathrm{H}$

$-7.26000900$

0.44953100

0.93808000

C

$-3.49904500 \quad-3.17176600$

$-0.37688200$

C

$-2.18199600-3.03481800$

0.10588300

C

$-3.78426200$

$-4.25105400$

$-1.24313600$

C

$-1.18127800-3.98905300$

$-0.11428100$

C

$\begin{array}{lll}-2.78113500 & -5.19798400 & -1.48863800\end{array}$

C

$-1.52077800 \quad-5.08964900$

$-0.91338000$

$\mathrm{H}$

$\begin{array}{lll}-0.77198100 & -5.85506900 & -1.09767200\end{array}$

$\mathrm{O}$

$-1.87836100-1.92420400$

0.88568500

$\mathrm{O}$

$-3.12618400$

$-0.40689800$

$-0.74098900$

$\mathrm{P}$

$-1.75501800$

$-0.45075200$

0.16820400

Ag

1.54200000

0.04788700

$-0.93421500$

$\mathrm{O}$

$-0.62675900$

$-0.40033700$

$-0.84207200$

$\mathrm{O}$

$-1.79883400$

0.55033900

1.29936000

$\mathrm{H}$

$\begin{array}{lll}-7.60721100 & -1.92812200 & 1.50344400\end{array}$

$\mathrm{H}$

$\begin{array}{lll}-2.99865100 & -6.03583600 & -2.14721300\end{array}$

C

4.34715000

2.99750300

$-0.24562300$

$\mathrm{O}$

5.3491020

2.64783900

$-0.86266700$

$\mathrm{N}$

4.34324000

4.04177900

0.68017400

C

5.49117500

4.90098100

0.85008700

$\mathrm{H}$

5.34509100

5.87742600

0.36147600

$\mathrm{H}$

5.70242300

5.07779900

1.91277800

$\mathrm{H}$

6.34329800

4.39881400

0.38679100

C

3.01639700

4.49487500

1.02192000

$\mathrm{H}$

2.8948750

4.64035700

2.10264200 


\begin{tabular}{|c|c|c|c|}
\hline $\mathrm{H}$ & 2.79307400 & 5.46490700 & 0.54865800 \\
\hline $\mathrm{C}$ & 2.05970900 & 3.40082100 & 0.45480000 \\
\hline $\mathrm{C}$ & 1.28402800 & 2.53502600 & 1.43725100 \\
\hline $\mathrm{C}$ & 0.95580700 & 3.91630200 & -0.43941600 \\
\hline $\mathrm{N}$ & 0.03727500 & 2.44168500 & 1.02909200 \\
\hline $\mathrm{C}$ & -0.22516300 & 3.25905900 & -0.10051200 \\
\hline $\mathrm{H}$ & -0.68073900 & 1.72538700 & 1.32683800 \\
\hline $\mathrm{C}$ & 0.96403600 & 4.79213600 & -1.51840600 \\
\hline $\mathrm{H}$ & 1.87223500 & 5.30868600 & -1.81729500 \\
\hline $\mathrm{C}$ & -0.23013700 & 4.98375000 & -2.22663100 \\
\hline $\mathrm{H}$ & -0.24477300 & 5.66644300 & -3.07161500 \\
\hline $\mathrm{C}$ & -1.40065100 & 4.30133300 & -1.87182100 \\
\hline $\mathrm{H}$ & -2.31130000 & 4.45242000 & -2.44243800 \\
\hline $\mathrm{C}$ & -1.42055300 & 3.41428100 & -0.78978200 \\
\hline $\mathrm{H}$ & -2.31904700 & 2.87730200 & -0.50795200 \\
\hline $\mathrm{C}$ & 1.72005100 & 1.84024300 & 2.72365000 \\
\hline $\mathrm{C}$ & 1.33488200 & 0.33959500 & 2.69676200 \\
\hline $\mathrm{H}$ & 0.27009200 & 0.17613000 & 2.51304400 \\
\hline $\mathrm{H}$ & 1.91058700 & -0.19870700 & 1.93587300 \\
\hline $\mathrm{H}$ & 1.58022900 & -0.10446300 & 3.66782400 \\
\hline $\mathrm{C}$ & 3.22770200 & 1.94591200 & 3.02599400 \\
\hline $\mathrm{H}$ & 3.56401700 & 2.97593400 & 3.16282500 \\
\hline $\mathrm{H}$ & 3.41974600 & 1.41376700 & 3.96405200 \\
\hline $\mathrm{H}$ & 3.84092500 & 1.48313200 & 2.25237000 \\
\hline $\mathrm{C}$ & 0.93464800 & 2.54050300 & 3.87117200 \\
\hline $\mathrm{H}$ & 1.14981400 & 3.61468900 & 3.91297500 \\
\hline $\mathrm{H}$ & -0.14596200 & 2.40936400 & 3.75938700 \\
\hline $\mathrm{H}$ & 1.23584100 & 2.09990600 & 4.82761900 \\
\hline $\mathrm{C}$ & -5.10947300 & -4.40792700 & -1.95734200 \\
\hline $\mathrm{H}$ & -5.70252800 & -5.23125400 & -1.53987300 \\
\hline $\mathrm{H}$ & -5.71573300 & -3.50090100 & -1.90846700 \\
\hline $\mathrm{H}$ & -4.93822900 & -4.64377300 & -3.01392800 \\
\hline
\end{tabular}




$\begin{array}{llll}\mathrm{C} & -5.99078000 & -4.02091800 & 1.12330200 \\ \mathrm{H} & -6.47678400 & -4.03681000 & 2.10504800 \\ \mathrm{H} & -6.66378900 & -4.53517900 & 0.42596200 \\ \mathrm{H} & -5.07230400 & -4.60851700 & 1.18954100 \\ \mathrm{C} & 0.18943100 & -3.86081200 & 0.46961500 \\ \mathrm{C} & 0.38878700 & -4.02231900 & 1.85647500 \\ \mathrm{C} & 1.29567900 & -3.61699700 & -0.37531900 \\ \mathrm{C} & 1.68682900 & -3.93213200 & 2.37405300 \\ \mathrm{C} & 2.57481600 & -3.53653600 & 0.18563300 \\ \mathrm{C} & 2.79409500 & -3.6885500 & 1.55935100 \\ \mathrm{H} & 1.83342800 & -4.06215300 & 3.44480500 \\ \mathrm{H} & 3.42155700 & -3.35072600 & -0.47071100 \\ \mathrm{C} & -5.11134100 & 1.62465000 & -0.15080900 \\ \mathrm{C} & -5.22742300 & 2.10700400 & -1.47161100 \\ \mathrm{C} & -4.92851100 & 2.53344600 & 0.91597800 \\ \mathrm{C} & -5.18011400 & 3.48793500 & -1.70045900 \\ \mathrm{C} & -4.88544700 & 3.90458200 & 0.63993100 \\ \mathrm{C} & -5.01491700 & 4.40545700 & -0.65918100 \\ \mathrm{H} & -5.29183800 & 3.85356900 & -2.71989500 \\ \mathrm{H} & -4.74868400 & 4.60026200 & 1.46599500 \\ \mathrm{C} & 4.18915100 & -3.59196500 & 2.13359200 \\ \mathrm{H} & 4.18127100 & -3.70718300 & 3.22248600 \\ \mathrm{H} & 4.84754200 & -4.36682900 & 1.72076100 \\ \mathrm{H} & 4.64953900 & -2.62422200 & 1.89990000 \\ \mathrm{C} & -0.76588200 & -4.29462000 & 2.79436200 \\ \mathrm{H} & -1.41020300 & -3.41349900 & 2.89437900 \\ \mathrm{H} & -1.39576200 & -5.11578000 & 2.43326200 \\ \mathrm{H} & -0.40147300 & -4.56129600 & 3.79169000 \\ & -5.41556200 & 1.16206900 & -2.63689600 \\ -4.53048200 & 0.53101000 & -2.77875800 \\ \mathrm{H} & -6.26765400 & 0.49060900 & -2.47916400 \\ & -5.58820000 & 1.71627500 & -3.56495600\end{array}$




\begin{tabular}{|c|c|c|c|}
\hline $\mathrm{C}$ & -4.74908500 & 2.05271900 & 2.33939800 \\
\hline $\mathrm{H}$ & -4.59211000 & 2.90018100 & 3.01497700 \\
\hline $\mathrm{H}$ & -5.61992300 & 1.49447900 & 2.70070000 \\
\hline $\mathrm{H}$ & -3.88210600 & 1.38645200 & 2.41197700 \\
\hline $\mathrm{C}$ & -4.94706100 & 5.89136700 & -0.92813200 \\
\hline $\mathrm{H}$ & -5.43025600 & 6.46620400 & -0.13009200 \\
\hline $\mathrm{H}$ & -3.90606300 & 6.23700800 & -0.99001000 \\
\hline $\mathrm{H}$ & -5.43473300 & 6.15051500 & -1.87409300 \\
\hline $\mathrm{C}$ & 1.12856900 & -3.43138900 & -1.86971700 \\
\hline $\mathrm{H}$ & 0.28921300 & -2.76584500 & -2.09674700 \\
\hline $\mathrm{H}$ & 2.03594000 & -3.00129000 & -2.30515100 \\
\hline $\mathrm{H}$ & 0.93186500 & -4.38200300 & -2.38148300 \\
\hline $\mathrm{C}$ & 2.98924600 & 2.40053600 & -0.32541700 \\
\hline $\mathrm{H}$ & 2.69208800 & 2.33692000 & -1.37564100 \\
\hline $\mathrm{C}$ & 7.18109600 & -2.88796600 & -2.31306500 \\
\hline $\mathrm{C}$ & 5.96490800 & -2.24215500 & -2.10424800 \\
\hline C & 5.83634500 & -1.30790100 & -1.06611800 \\
\hline $\mathrm{C}$ & 6.93688700 & -1.01483700 & -0.24765700 \\
\hline $\mathrm{C}$ & 8.15256200 & -1.66117700 & -0.46288800 \\
\hline $\mathrm{C}$ & 8.27486200 & -2.59921300 & -1.49162200 \\
\hline $\mathrm{H}$ & 7.27864400 & -3.61360700 & -3.11570500 \\
\hline $\mathrm{H}$ & 5.10749700 & -2.44611700 & -2.73689100 \\
\hline $\mathrm{H}$ & 6.82975000 & -0.27225400 & 0.53460400 \\
\hline $\mathrm{H}$ & 9.00670100 & -1.43005100 & 0.16745700 \\
\hline $\mathrm{H}$ & 9.22416400 & -3.10214200 & -1.65666500 \\
\hline $\mathrm{C}$ & 4.53246200 & -0.62822700 & -0.85617700 \\
\hline $\mathrm{O}$ & 3.60142100 & -0.74144100 & -1.67335500 \\
\hline $\mathrm{O}$ & 4.42725900 & 0.06724900 & 0.25673400 \\
\hline & 3.71071100 & 0.83097600 & 0.13037500 \\
\hline
\end{tabular}




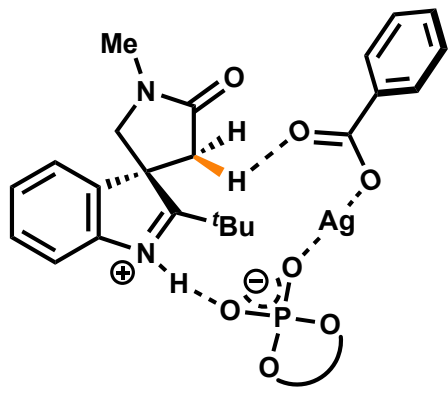

CP5

Zero-point correction $=$

1.015962 (Hartree/Particle)

Thermal correction to Energy=

1.082141

Thermal correction to Enthalpy=

1.083086

Thermal correction to Gibbs Free Energy=

0.907596

Sum of electronic and zero-point Energies= $-3253.166335$

Sum of electronic and thermal Energies= $-3253.100155$

Sum of electronic and thermal Enthalpies= $-3253.099211$

Sum of electronic and thermal Free Energies= $-3253.274701$

Cartesian Coordinates

\begin{tabular}{lrrr} 
Atom & X & Y & $Z$ \\
\hline C & -4.46576400 & 2.17968800 & 0.33265600 \\
C & -5.53970400 & 2.50842300 & 1.17236600 \\
C & -6.29654000 & 1.52161200 & 1.79126900 \\
C & -6.06296400 & 0.16108900 & 1.55250200 \\
C & -5.04108600 & -0.20310900 & 0.64698400 \\
C & -4.22493400 & 0.81781300 & 0.11463500 \\
H & -5.76172200 & 3.55684800 & 1.35239100 \\
C & -4.80049200 & -1.62130700 & 0.24054600 \\
C & -3.51901800 & -2.18272100 & 0.40696100 \\
C & -5.80912900 & -2.41979100 & -0.34608300 \\
C & -3.21820200 & -3.51677000 & 0.10520500 \\
C & -5.51940600 & -3.75884000 & -0.63883700 \\
C & -4.26297100 & -4.30227900 & -0.40003700
\end{tabular}


Supporting Information

\begin{tabular}{|c|c|c|c|}
\hline $\mathrm{H}$ & -4.06934000 & -5.34609400 & -0.63145200 \\
\hline $\mathrm{O}$ & -2.51487500 & -1.37911500 & 0.94331700 \\
\hline $\mathrm{O}$ & -3.15625900 & 0.45454700 & -0.70249200 \\
\hline $\mathrm{P}$ & -1.83381400 & -0.21565800 & 0.01205200 \\
\hline $\mathrm{Ag}$ & 0.94125500 & -1.74006100 & -1.07322600 \\
\hline $\mathrm{O}$ & -1.02888000 & -0.78523600 & -1.13894800 \\
\hline $\mathrm{O}$ & -1.16904300 & 0.74734500 & 0.97078000 \\
\hline $\mathrm{H}$ & -7.08895400 & 1.80691200 & 2.47945500 \\
\hline $\mathrm{H}$ & -6.29592800 & -4.38023800 & -1.07899100 \\
\hline $\mathrm{C}$ & 5.71807700 & 2.55544200 & 0.85228000 \\
\hline $\mathrm{O}$ & 6.85213500 & 2.11724100 & 0.95030400 \\
\hline $\mathrm{N}$ & 5.25380500 & 3.70868200 & 1.43897400 \\
\hline $\mathrm{C}$ & 6.09305600 & 4.57357400 & 2.23922400 \\
\hline $\mathrm{H}$ & 6.16227400 & 5.57694500 & 1.79886100 \\
\hline $\mathrm{H}$ & 5.70392100 & 4.66817800 & 3.26175200 \\
\hline $\mathrm{H}$ & 7.08710700 & 4.12266100 & 2.27099300 \\
\hline $\mathrm{C}$ & 3.86338800 & 4.01151300 & 1.18373200 \\
\hline $\mathrm{H}$ & 3.31986000 & 4.18614300 & 2.12071900 \\
\hline $\mathrm{H}$ & 3.75728600 & 4.92087700 & 0.57673600 \\
\hline $\mathrm{C}$ & 3.30428400 & 2.77361600 & 0.38791000 \\
\hline $\mathrm{C}$ & 2.25744500 & 1.94901100 & 1.16271800 \\
\hline $\mathrm{C}$ & 2.52094400 & 3.15813100 & -0.85053800 \\
\hline $\mathrm{N}$ & 1.16496700 & 1.87863600 & 0.45148600 \\
\hline $\mathrm{C}$ & 1.25684300 & 2.57777300 & -0.77748600 \\
\hline $\mathrm{H}$ & 0.26381200 & 1.36825200 & 0.71338400 \\
\hline $\mathrm{C}$ & 2.86616000 & 3.90574400 & -1.96915900 \\
\hline $\mathrm{H}$ & 3.84682500 & 4.36519000 & -2.06274300 \\
\hline $\mathrm{C}$ & 1.91551600 & 4.04973200 & -2.98955600 \\
\hline $\mathrm{H}$ & 2.16700000 & 4.62818300 & -3.87377900 \\
\hline $\mathrm{C}$ & 0.65097800 & 3.45660300 & -2.89029900 \\
\hline $\mathrm{H}$ & -0.06887900 & 3.58360300 & -3.69248400 \\
\hline $\mathrm{C}$ & 0.29225300 & 2.70166000 & -1.76846300 \\
\hline
\end{tabular}




\begin{tabular}{|c|c|c|c|}
\hline $\mathrm{H}$ & -0.68590200 & 2.24413200 & -1.67865300 \\
\hline $\mathrm{C}$ & 2.30900100 & 1.34220500 & 2.55974500 \\
\hline $\mathrm{C}$ & 1.68746900 & -0.07850500 & 2.55333300 \\
\hline $\mathrm{H}$ & 0.63015600 & -0.06675100 & 2.27696000 \\
\hline $\mathrm{H}$ & 2.23569800 & -0.72725100 & 1.86518600 \\
\hline $\mathrm{H}$ & 1.76305200 & -0.48850400 & 3.56665000 \\
\hline $\mathrm{C}$ & 3.73861300 & 1.23731000 & 3.12334200 \\
\hline $\mathrm{H}$ & 4.24227400 & 2.20387900 & 3.20269400 \\
\hline $\mathrm{H}$ & 3.67803600 & 0.81641300 & 4.13217400 \\
\hline $\mathrm{H}$ & 4.35641800 & 0.56319400 & 2.52434500 \\
\hline $\mathrm{C}$ & 1.45208200 & 2.25900500 & 3.48192200 \\
\hline $\mathrm{H}$ & 1.84745900 & 3.27966600 & 3.54190200 \\
\hline $\mathrm{H}$ & 0.41303700 & 2.30584000 & 3.14206600 \\
\hline $\mathrm{H}$ & 1.46052200 & 1.83891000 & 4.49334900 \\
\hline $\mathrm{C}$ & -7.17542200 & -1.88535300 & -0.71819600 \\
\hline $\mathrm{H}$ & -7.94299400 & -2.18693400 & 0.00543700 \\
\hline $\mathrm{H}$ & -7.18977000 & -0.79489900 & -0.78103500 \\
\hline $\mathrm{H}$ & -7.48081600 & -2.28556500 & -1.69130700 \\
\hline $\mathrm{C}$ & -6.88792800 & -0.85244500 & 2.31594400 \\
\hline $\mathrm{H}$ & -6.96910100 & -0.55167800 & 3.36666900 \\
\hline $\mathrm{H}$ & -7.91061200 & -0.92309000 & 1.92514200 \\
\hline $\mathrm{H}$ & -6.44859700 & -1.85171200 & 2.28353100 \\
\hline $\mathrm{C}$ & -1.84212200 & -4.07814800 & 0.26919800 \\
\hline $\mathrm{C}$ & -1.31955900 & -4.33540300 & 1.55179800 \\
\hline $\mathrm{C}$ & -1.06294600 & -4.35591200 & -0.87839500 \\
\hline $\mathrm{C}$ & -0.02130400 & -4.84870200 & 1.66953800 \\
\hline $\mathrm{C}$ & 0.23244900 & -4.85782400 & -0.71266500 \\
\hline $\mathrm{C}$ & 0.77679800 & -5.10683100 & 0.55424600 \\
\hline $\mathrm{H}$ & 0.37649900 & -5.04433800 & 2.66357400 \\
\hline $\mathrm{H}$ & 0.83915600 & -5.05105400 & -1.59486100 \\
\hline $\mathrm{C}$ & -3.65286200 & 3.26766700 & -0.29733300 \\
\hline $\mathrm{C}$ & -3.80691200 & 3.56017800 & -1.66934400 \\
\hline
\end{tabular}




\begin{tabular}{|c|c|c|c|}
\hline $\mathrm{C}$ & -2.79360500 & 4.05905100 & 0.49671000 \\
\hline $\mathrm{C}$ & -3.11247900 & 4.64540700 & -2.21750000 \\
\hline $\mathrm{C}$ & -2.11958000 & 5.13331500 & -0.09381900 \\
\hline $\mathrm{C}$ & -2.26690000 & 5.44846300 & -1.44778900 \\
\hline $\mathrm{H}$ & -3.25050400 & 4.87606300 & -3.27248400 \\
\hline $\mathrm{H}$ & -1.46355800 & 5.74349200 & 0.52495500 \\
\hline $\mathrm{C}$ & 2.18985000 & -5.62159000 & 0.68947100 \\
\hline $\mathrm{H}$ & 2.48152500 & -5.72204200 & 1.74001800 \\
\hline $\mathrm{H}$ & 2.30630400 & -6.60428900 & 0.21435500 \\
\hline $\mathrm{H}$ & 2.89193900 & -4.93692800 & 0.20009700 \\
\hline $\mathrm{C}$ & -2.12910400 & -4.06174300 & 2.79950600 \\
\hline $\mathrm{H}$ & -2.25668500 & -2.98453500 & 2.95903000 \\
\hline $\mathrm{H}$ & -3.13212200 & -4.49939800 & 2.73617600 \\
\hline $\mathrm{H}$ & -1.63382900 & -4.47664900 & 3.68324500 \\
\hline $\mathrm{C}$ & -4.71743600 & 2.73523400 & -2.55100200 \\
\hline $\mathrm{H}$ & -4.33591600 & 1.71424800 & -2.66801800 \\
\hline $\mathrm{H}$ & -5.72557500 & 2.65526800 & -2.12805600 \\
\hline $\mathrm{H}$ & -4.80286600 & 3.18020000 & -3.54747700 \\
\hline $\mathrm{C}$ & -2.57408500 & 3.75459000 & 1.96277900 \\
\hline $\mathrm{H}$ & -1.80673100 & 4.41460100 & 2.38194800 \\
\hline $\mathrm{H}$ & -3.48608300 & 3.89125300 & 2.55522700 \\
\hline $\mathrm{H}$ & -2.25316100 & 2.71572100 & 2.09703300 \\
\hline $\mathrm{C}$ & -1.51374300 & 6.60552600 & -2.06259900 \\
\hline $\mathrm{H}$ & -1.42514800 & 7.44423000 & -1.36279300 \\
\hline $\mathrm{H}$ & -0.49426800 & 6.30964300 & -2.34532500 \\
\hline $\mathrm{H}$ & -2.01084000 & 6.97204600 & -2.96722600 \\
\hline $\mathrm{C}$ & -1.58551500 & -4.09448900 & -2.27610000 \\
\hline $\mathrm{H}$ & -1.93332700 & -3.06142900 & -2.38221600 \\
\hline $\mathrm{H}$ & -0.79836200 & -4.26706800 & -3.01741200 \\
\hline $\mathrm{H}$ & -2.42818400 & -4.74839800 & -2.52941000 \\
\hline $\mathrm{C}$ & 4.58834300 & 1.95025400 & 0.03584000 \\
\hline $\mathrm{H}$ & 4.82832100 & 2.07557700 & -1.02537400 \\
\hline
\end{tabular}




$\begin{array}{lrrr}\text { C } & 6.79699500 & -4.08424000 & -1.65772200 \\ \mathrm{C} & 5.48884900 & -3.61437800 & -1.54640800 \\ \mathrm{C} & 5.20755000 & -2.46577300 & -0.79236900 \\ \mathrm{C} & 6.25988000 & -1.79285800 & -0.15645400 \\ \mathrm{C} & 7.56900700 & -2.26042700 & -0.27124100 \\ \mathrm{C} & 7.84044700 & -3.40830400 & -1.01975200 \\ \mathrm{H} & 7.00371600 & -4.97619200 & -2.24397700 \\ \mathrm{H} & 4.66893600 & -4.12339000 & -2.04109600 \\ \mathrm{H} & 6.05260100 & -0.89835300 & 0.42052500 \\ \mathrm{H} & 8.37642000 & -1.72594500 & 0.22239200 \\ \mathrm{H} & 8.86093900 & -3.77299400 & -1.10793400 \\ \mathrm{C} & 3.78916700 & -1.95765000 & -0.67645100 \\ \mathrm{O} & 2.89944100 & -2.63744200 & -1.30234400 \\ \mathrm{O} & 3.54915200 & -0.93061100 & 0.00389200 \\ \mathrm{H} & 4.46975800 & 0.87482800 & 0.19610200\end{array}$

\section{$\mathrm{TS}_{\text {MAJOR }}$}

Zero-point correction=

Thermal correction to Energy=

Thermal correction to Enthalpy=

Thermal correction to Gibbs Free Energy=

Sum of electronic and zero-point Energies=

Sum of electronic and thermal Energies=

Sum of electronic and thermal Enthalpies=

Sum of electronic and thermal Free Energies=
1.090299

1.091243

0.914413

$-3174.202056$

$-3174.134945$

$-3174.134001$

$-3174.310831$

Cartesian Coordinates

$\begin{array}{llll}\text { Atom } & \mathrm{X} & \mathrm{Y} & \mathrm{Z}\end{array}$
$\mathrm{C}$
4.44021500
$1.26234500 \quad-0.86676800$
$\mathrm{C}$
$\begin{array}{llll}5.60336400 & 1.11927600 & -1.63593900\end{array}$
C
$\begin{array}{lll}6.12594900 & -0.13694300 & -1.91986800\end{array}$ 
Supporting Information

\begin{tabular}{|c|c|c|c|}
\hline $\mathrm{C}$ & 5.55294000 & -1.30599300 & -1.40155400 \\
\hline $\mathrm{C}$ & 4.42600600 & -1.19175500 & -0.55683800 \\
\hline $\mathrm{C}$ & 3.86394200 & 0.08826700 & -0.36657200 \\
\hline $\mathrm{H}$ & 6.08386400 & 2.01012200 & -2.03158500 \\
\hline $\mathrm{C}$ & 3.81281900 & -2.36789800 & 0.13518700 \\
\hline $\mathrm{C}$ & 2.43980700 & -2.63995400 & -0.04153100 \\
\hline $\mathrm{C}$ & 4.55435100 & -3.20270900 & 1.00236900 \\
\hline $\mathrm{C}$ & 1.79938000 & -3.75060100 & 0.52447400 \\
\hline $\mathrm{C}$ & 3.92133800 & -4.31839400 & 1.56585700 \\
\hline $\mathrm{C}$ & 2.58347700 & -4.60019300 & 1.31661200 \\
\hline $\mathrm{H}$ & 2.12156100 & -5.47846900 & 1.75975000 \\
\hline $\mathrm{O}$ & 1.70121700 & -1.79107100 & -0.85539500 \\
\hline $\mathrm{O}$ & 2.70227800 & 0.19044000 & 0.39101100 \\
\hline $\mathrm{P}$ & 1.28939700 & -0.29333100 & -0.30387900 \\
\hline $\mathrm{Ag}$ & -1.80468100 & -0.08937600 & 0.87888300 \\
\hline $\mathrm{O}$ & 0.33550000 & -0.40490200 & 0.87771200 \\
\hline $\mathrm{O}$ & 0.92098100 & 0.51464300 & -1.51309900 \\
\hline $\mathrm{H}$ & 6.99887100 & -0.22016700 & -2.56345800 \\
\hline $\mathrm{H}$ & 4.49148500 & -4.96924700 & 2.22488600 \\
\hline $\mathrm{C}$ & -5.01187400 & -0.48585600 & 0.11119600 \\
\hline $\mathrm{O}$ & -5.39018400 & -1.52318100 & 0.66165400 \\
\hline $\mathrm{N}$ & -5.54180200 & -0.04401300 & -1.07217800 \\
\hline $\mathrm{C}$ & -6.72732500 & -0.73030200 & -1.57937600 \\
\hline $\mathrm{H}$ & -7.64031400 & -0.16901600 & -1.33537400 \\
\hline $\mathrm{H}$ & -6.66347500 & -0.83407400 & -2.66713300 \\
\hline $\mathrm{H}$ & -6.78436500 & -1.71418700 & -1.11662200 \\
\hline $\mathrm{C}$ & -5.22349300 & 1.26744100 & -1.67437300 \\
\hline $\mathrm{H}$ & -5.57906300 & 1.21458500 & -2.70756000 \\
\hline $\mathrm{H}$ & -5.82585000 & 2.04835300 & -1.18649500 \\
\hline $\mathrm{C}$ & -3.77383600 & 1.64923000 & -1.59785800 \\
\hline $\mathrm{C}$ & -2.65472600 & 1.05105200 & -2.20984300 \\
\hline $\mathrm{C}$ & -3.29435700 & 2.83211100 & -0.91326800 \\
\hline
\end{tabular}




\begin{tabular}{|c|c|c|c|}
\hline $\mathrm{N}$ & -1.54064000 & 1.76769900 & -1.85211800 \\
\hline $\mathrm{C}$ & -1.88573200 & 2.84496900 & -1.06132700 \\
\hline $\mathrm{H}$ & -0.57864000 & 1.40247000 & -1.91131600 \\
\hline $\mathrm{C}$ & -3.90720900 & 3.86030500 & -0.17372500 \\
\hline $\mathrm{H}$ & -4.98786200 & 3.90090900 & -0.05516200 \\
\hline $\mathrm{C}$ & -3.10780000 & 4.84866200 & 0.39374300 \\
\hline $\mathrm{H}$ & -3.57246600 & 5.65435400 & 0.95596000 \\
\hline $\mathrm{C}$ & -1.70539200 & 4.83057800 & 0.24335100 \\
\hline $\mathrm{H}$ & -1.10892700 & 5.61721600 & 0.69702700 \\
\hline $\mathrm{C}$ & -1.07296300 & 3.82722800 & -0.48527400 \\
\hline $\mathrm{H}$ & 0.00567200 & 3.80904100 & -0.61226100 \\
\hline $\mathrm{C}$ & -2.50018700 & -0.12993900 & -3.17222200 \\
\hline $\mathrm{C}$ & -1.31367200 & 0.13286400 & -4.13351200 \\
\hline $\mathrm{H}$ & -1.44407400 & 1.07272600 & -4.68208300 \\
\hline $\mathrm{H}$ & -0.35588800 & 0.16250500 & -3.60735700 \\
\hline $\mathrm{H}$ & -1.26075600 & -0.68158600 & -4.86486800 \\
\hline $\mathrm{C}$ & -2.22323100 & -1.44101700 & -2.39954600 \\
\hline $\mathrm{H}$ & -3.03641300 & -1.68635200 & -1.70985500 \\
\hline $\mathrm{H}$ & -2.12284100 & -2.27274200 & -3.10684000 \\
\hline $\mathrm{H}$ & -1.29004600 & -1.38092800 & -1.83280300 \\
\hline $\mathrm{C}$ & -3.76529700 & -0.31764300 & -4.03462500 \\
\hline $\mathrm{H}$ & -4.61518100 & -0.66374400 & -3.44234200 \\
\hline $\mathrm{H}$ & -4.04450300 & 0.60702700 & -4.55327000 \\
\hline $\mathrm{H}$ & -3.57079100 & -1.08116300 & -4.79592800 \\
\hline $\mathrm{C}$ & 5.99051200 & -2.92286400 & 1.38990100 \\
\hline $\mathrm{H}$ & 6.69496100 & -3.55120700 & 0.83068700 \\
\hline $\mathrm{H}$ & 6.26872400 & -1.88063600 & 1.21707600 \\
\hline $\mathrm{H}$ & 6.14136900 & -3.14359600 & 2.45242500 \\
\hline $\mathrm{C}$ & 6.14168700 & -2.63755100 & -1.81543900 \\
\hline $\mathrm{H}$ & 6.33848900 & -2.63994300 & -2.89364400 \\
\hline $\mathrm{H}$ & 7.09931700 & -2.83287600 & -1.31675600 \\
\hline $\mathrm{H}$ & 5.47294600 & -3.47179900 & -1.5927540 \\
\hline
\end{tabular}




\begin{tabular}{|c|c|c|c|}
\hline $\mathrm{C}$ & 0.34091900 & -4.02515800 & 0.32692200 \\
\hline $\mathrm{C}$ & -0.13360100 & -4.51989200 & -0.90373400 \\
\hline $\mathrm{C}$ & -0.56228800 & -3.81717600 & 1.39426400 \\
\hline $\mathrm{C}$ & -1.50011900 & -4.79345400 & -1.04877300 \\
\hline $\mathrm{C}$ & -1.91969200 & -4.09114900 & 1.20132900 \\
\hline $\mathrm{C}$ & -2.41302900 & -4.57991300 & -0.01419100 \\
\hline $\mathrm{H}$ & -1.85772100 & -5.18346500 & -2.00009800 \\
\hline $\mathrm{H}$ & -2.61218200 & -3.92225400 & 2.02438000 \\
\hline $\mathrm{C}$ & 3.86665000 & 2.61885500 & -0.59617200 \\
\hline $\mathrm{C}$ & 3.99919500 & 3.19753500 & 0.68365200 \\
\hline $\mathrm{C}$ & 3.22845300 & 3.33882500 & -1.62980100 \\
\hline $\mathrm{C}$ & 3.49735500 & 4.48478700 & 0.90726400 \\
\hline $\mathrm{C}$ & 2.74249000 & 4.62404700 & -1.36181800 \\
\hline $\mathrm{C}$ & 2.86970100 & 5.21880800 & -0.10240800 \\
\hline $\mathrm{H}$ & 3.60833600 & 4.92645600 & 1.89604700 \\
\hline $\mathrm{H}$ & 2.25161700 & 5.17469000 & -2.16255800 \\
\hline $\mathrm{C}$ & -3.88754000 & -4.86448400 & -0.18733100 \\
\hline $\mathrm{H}$ & -4.11253000 & -5.19170500 & -1.20806300 \\
\hline $\mathrm{H}$ & -4.22448200 & -5.65513200 & 0.49587200 \\
\hline $\mathrm{H}$ & -4.49102400 & -3.97414900 & 0.02774900 \\
\hline $\mathrm{C}$ & 0.80314600 & -4.75470600 & -2.06730200 \\
\hline $\mathrm{H}$ & 1.18801200 & -3.80593200 & -2.45957300 \\
\hline $\mathrm{H}$ & 1.67073400 & -5.35716400 & -1.77419900 \\
\hline $\mathrm{H}$ & 0.28987300 & -5.27434900 & -2.88292400 \\
\hline $\mathrm{C}$ & 4.67092100 & 2.45392000 & 1.81625500 \\
\hline $\mathrm{H}$ & 4.07074800 & 1.59341300 & 2.13441600 \\
\hline $\mathrm{H}$ & 5.65398100 & 2.06911700 & 1.52128100 \\
\hline $\mathrm{H}$ & 4.80935200 & 3.10781800 & 2.68335600 \\
\hline $\mathrm{C}$ & 3.02925700 & 2.73860300 & -3.00403800 \\
\hline $\mathrm{H}$ & 2.48543100 & 3.43343700 & -3.65259600 \\
\hline $\mathrm{H}$ & 3.98092400 & 2.50163900 & -3.49317000 \\
\hline $\mathrm{H}$ & 2.45508900 & 1.80845200 & -2.93504600 \\
\hline
\end{tabular}




$\begin{array}{lrrr}\mathrm{C} & 2.37260800 & 6.62474800 & 0.14711800 \\ \mathrm{H} & 2.16127600 & 6.79305000 & 1.20881100 \\ \mathrm{H} & 3.11774000 & 7.37228000 & -0.15773000 \\ \mathrm{H} & 1.45798000 & 6.83449100 & -0.41932300 \\ \mathrm{C} & -0.09242000 & -3.28501300 & 2.73083000 \\ \mathrm{H} & 0.45200400 & -2.34344800 & 2.60296300 \\ \mathrm{H} & -0.94569400 & -3.10556600 & 3.39414300 \\ \mathrm{H} & 0.58105800 & -3.98409600 & 3.24030400 \\ \mathrm{C} & -3.90715900 & 0.34449400 & 0.73582200 \\ \mathrm{H} & -4.15220300 & 1.38933300 & 0.91954300 \\ \mathrm{~N} & -4.34143300 & -0.10003000 & 2.52040200 \\ \mathrm{~N} & -4.29929900 & -0.35722200 & 3.59599100 \\ \mathrm{C} & -0.41614100 & 0.62131600 & 4.21869500 \\ \mathrm{O} & -1.52082200 & 1.13828100 & 3.46502700 \\ \mathrm{C} & -1.36970300 & 2.56629000 & 3.47224500 \\ \mathrm{C} & 0.15201700 & 2.84828800 & 3.47382600 \\ \mathrm{C} & 0.78615400 & 1.46637200 & 3.78295000 \\ \mathrm{H} & -0.62123900 & 0.73276600 & 5.29628800 \\ \mathrm{H} & -0.32285400 & -0.44036600 & 3.98203700 \\ \mathrm{H} & -1.88856000 & 2.95830200 & 2.59455900 \\ \mathrm{H} & -1.84723400 & 2.97540600 & 4.37619900 \\ \mathrm{H} & 0.49032000 & 3.22778100 & 2.50594400 \\ \mathrm{H} & 0.40710300 & 3.59689700 & 4.23110700 \\ \mathrm{H} & 1.22343300 & 1.03525400 & 2.87940700 \\ \mathrm{H} & -5522700 & 1.51744200 & 4.55623700\end{array}$

\section{$\mathrm{TS}_{\text {MINOR }}$}

Zero-point correction=

Thermal correction to Energy=

Thermal correction to Enthalpy=

Thermal correction to Gibbs Free Energy=

Sum of electronic and zero-point Energies=
1.023106 (Hartree/Particle)

1.090293

1.091237

0.912923

$-3174.200244$ 
Sum of electronic and thermal Energies=

$-3174.133057$

Sum of electronic and thermal Enthalpies=

$-3174.132113$

Sum of electronic and thermal Free Energies=

\section{Cartesian Coordinates}

\begin{tabular}{|c|c|c|c|}
\hline Atom & $\mathrm{X}$ & $\mathrm{Y}$ & $\mathrm{Z}$ \\
\hline $\mathrm{O}$ & -5.22597300 & 1.10745300 & 1.99995500 \\
\hline $\mathrm{N}$ & -4.59878600 & -2.12633200 & 2.35688300 \\
\hline $\mathrm{N}$ & -4.53686100 & -1.31055700 & 1.61028200 \\
\hline $\mathrm{N}$ & -1.54181500 & 0.99308400 & -2.35555900 \\
\hline $\mathrm{N}$ & -5.28879200 & 1.97743300 & -0.10694600 \\
\hline $\mathrm{C}$ & -3.05064600 & 4.29824600 & -1.37934000 \\
\hline $\mathrm{C}$ & -1.50594500 & 3.09691600 & 0.19210900 \\
\hline $\mathrm{C}$ & -0.74710400 & 3.69003900 & -2.12598300 \\
\hline $\mathrm{C}$ & -1.95726100 & 3.21538700 & -1.28401000 \\
\hline $\mathrm{C}$ & -1.63786900 & -1.23987300 & -3.50744400 \\
\hline $\mathrm{C}$ & -2.52995500 & -2.20006000 & -3.97240400 \\
\hline $\mathrm{C}$ & -3.92164100 & -2.04275900 & -3.80605500 \\
\hline $\mathrm{C}$ & -4.44989600 & -0.92721000 & -3.16232400 \\
\hline $\mathrm{C}$ & -2.17760700 & -0.11519900 & -2.87566200 \\
\hline $\mathrm{C}$ & -3.56887700 & 0.05892700 & -2.68192700 \\
\hline $\mathrm{C}$ & -2.45183500 & 1.86860000 & -1.82072300 \\
\hline $\mathrm{C}$ & -3.73408300 & 1.30020000 & -1.95479000 \\
\hline $\mathrm{C}$ & -5.07363300 & 1.84876400 & -1.56405900 \\
\hline $\mathrm{C}$ & -6.29472400 & 2.95031800 & 0.31351100 \\
\hline $\mathrm{C}$ & -4.88242100 & 1.05063500 & 0.81404000 \\
\hline $\mathrm{C}$ & -3.94694000 & -0.05002200 & 0.35434100 \\
\hline $\mathrm{H}$ & -2.62269600 & 5.26652500 & -1.09691900 \\
\hline $\mathrm{H}$ & -3.44092100 & 4.39189900 & -2.39938000 \\
\hline $\mathrm{H}$ & -3.88094900 & 4.10037500 & -0.69688100 \\
\hline $\mathrm{H}$ & -0.68934600 & 2.37693800 & 0.30218700 \\
\hline
\end{tabular}




\begin{tabular}{|c|c|c|c|}
\hline $\mathrm{H}$ & -1.13945200 & 4.06893900 & 0.54457800 \\
\hline $\mathrm{H}$ & -2.33599400 & 2.79458700 & 0.84024000 \\
\hline $\mathrm{H}$ & -0.44173100 & 4.68613000 & -1.78712300 \\
\hline $\mathrm{H}$ & 0.11743500 & 3.03136300 & -2.01099200 \\
\hline $\mathrm{H}$ & -1.00272500 & 3.75370700 & -3.18977100 \\
\hline $\mathrm{H}$ & -0.56425700 & -1.35132500 & -3.62342200 \\
\hline $\mathrm{H}$ & -2.14919200 & -3.08378800 & -4.47611100 \\
\hline $\mathrm{H}$ & -4.59338000 & -2.80283200 & -4.19631500 \\
\hline $\mathrm{H}$ & -5.52714800 & -0.82167600 & -3.05289100 \\
\hline $\mathrm{H}$ & -0.52550200 & 1.01337700 & -2.17363200 \\
\hline $\mathrm{H}$ & -5.24331400 & 2.84719900 & -1.97713500 \\
\hline $\mathrm{H}$ & -5.86030900 & 1.20784000 & -1.98860100 \\
\hline $\mathrm{H}$ & -6.06477900 & 3.93055100 & -0.11617100 \\
\hline $\mathrm{H}$ & -7.29695600 & 2.64853300 & -0.02162000 \\
\hline $\mathrm{H}$ & -6.29070000 & 3.01085800 & 1.40047400 \\
\hline $\mathrm{H}$ & -4.29539200 & -0.67047000 & -0.46831700 \\
\hline $\mathrm{Ag}$ & -1.83500800 & -0.18044500 & 0.81024800 \\
\hline $\mathrm{C}$ & 1.90912100 & -3.60794200 & 0.53480600 \\
\hline $\mathrm{C}$ & 2.65131300 & -4.44376800 & 1.38027700 \\
\hline C & 3.95261400 & -4.12262500 & 1.74648100 \\
\hline $\mathrm{C}$ & 4.59494600 & -2.98289300 & 1.24558300 \\
\hline $\mathrm{C}$ & 3.90154100 & -2.16078300 & 0.32802900 \\
\hline $\mathrm{C}$ & 2.55393600 & -2.46503500 & 0.04249000 \\
\hline $\mathrm{H}$ & 2.18429600 & -5.34472700 & 1.76900800 \\
\hline $\mathrm{C}$ & 4.54258100 & -0.97470200 & -0.31856800 \\
\hline $\mathrm{C}$ & 3.94660800 & 0.29577400 & -0.17457500 \\
\hline $\mathrm{C}$ & 5.72972100 & -1.07555300 & -1.07834000 \\
\hline $\mathrm{C}$ & 4.54336300 & 1.47688900 & -0.63297900 \\
\hline $\mathrm{C}$ & 6.32195700 & 0.10009300 & -1.55776500 \\
\hline C & 5.76169700 & 1.34826900 & -1.31478300 \\
\hline $\mathrm{H}$ & 6.25724300 & 2.24587000 & -1.67493800 \\
\hline $\mathrm{O}$ & 2.73276400 & 0.37905900 & 0.4975360 \\
\hline
\end{tabular}




\begin{tabular}{|c|c|c|c|}
\hline $\mathrm{O}$ & 1.85400800 & -1.62349000 & -0.81086500 \\
\hline $\mathrm{P}$ & 1.37537700 & -0.13425500 & -0.28323100 \\
\hline $\mathrm{O}$ & 1.07239200 & 0.67218600 & -1.51229700 \\
\hline $\mathrm{O}$ & 0.35234600 & -0.26139800 & 0.83597700 \\
\hline $\mathrm{H}$ & 4.48636100 & -4.76371500 & 2.44442500 \\
\hline $\mathrm{H}$ & 7.24056800 & 0.02875200 & -2.13587800 \\
\hline $\mathrm{C}$ & 3.94528900 & 2.83089800 & -0.40944200 \\
\hline $\mathrm{C}$ & 3.97672400 & 3.41283200 & 0.87603300 \\
\hline $\mathrm{C}$ & 3.40012500 & 3.55168500 & -1.49347800 \\
\hline $\mathrm{C}$ & 3.47292700 & 4.70556100 & 1.05259600 \\
\hline $\mathrm{C}$ & 2.90987700 & 4.84389200 & -1.27085500 \\
\hline $\mathrm{C}$ & 2.94068700 & 5.44258200 & -0.00881800 \\
\hline $\mathrm{H}$ & 3.50400100 & 5.14921100 & 2.04638600 \\
\hline $\mathrm{H}$ & 2.49045700 & 5.39533800 & -2.11057800 \\
\hline $\mathrm{C}$ & 0.49117600 & -3.94325000 & 0.19444800 \\
\hline $\mathrm{C}$ & 0.15964400 & -4.40655200 & -1.09778200 \\
\hline $\mathrm{C}$ & -0.51081800 & -3.84428500 & 1.18206300 \\
\hline $\mathrm{C}$ & -1.16387900 & -4.75551100 & -1.37838700 \\
\hline $\mathrm{C}$ & -1.82632900 & -4.20044900 & 0.85461600 \\
\hline $\mathrm{C}$ & -2.17621900 & -4.65468000 & -0.41893000 \\
\hline $\mathrm{H}$ & -1.41080900 & -5.11633500 & -2.37513800 \\
\hline $\mathrm{H}$ & -2.59270200 & -4.13246100 & 1.62512800 \\
\hline $\mathrm{C}$ & 3.30134300 & 2.94392900 & -2.87574700 \\
\hline $\mathrm{H}$ & 2.80868100 & 3.63643300 & -3.56635700 \\
\hline $\mathrm{H}$ & 4.28531700 & 2.70149200 & -3.29314400 \\
\hline $\mathrm{H}$ & 2.72086800 & 2.01524500 & -2.84542000 \\
\hline $\mathrm{C}$ & 4.54497500 & 2.66630100 & 2.06217100 \\
\hline $\mathrm{H}$ & 3.92127000 & 1.80262400 & 2.32058200 \\
\hline $\mathrm{H}$ & 5.55230300 & 2.28569400 & 1.85643700 \\
\hline $\mathrm{H}$ & 4.60234200 & 3.31711300 & 2.94072100 \\
\hline $\mathrm{C}$ & 2.44202000 & 6.85484700 & 0.19756900 \\
\hline $\mathrm{H}$ & 3.25668600 & 7.58550300 & 0.0995310 \\
\hline
\end{tabular}


Supporting Information

\begin{tabular}{|c|c|c|c|}
\hline $\mathrm{H}$ & 1.67785300 & 7.12285000 & -0.54048500 \\
\hline $\mathrm{H}$ & 2.00995900 & 6.98699400 & 1.19597700 \\
\hline $\mathrm{C}$ & -0.19824200 & -3.35580300 & 2.58009000 \\
\hline $\mathrm{H}$ & -1.11941100 & -3.17028500 & 3.14176200 \\
\hline $\mathrm{H}$ & 0.39538300 & -4.08390500 & 3.14630300 \\
\hline $\mathrm{H}$ & 0.37362800 & -2.42360400 & 2.54995800 \\
\hline C & 1.21018300 & -4.53459100 & -2.17755900 \\
\hline $\mathrm{H}$ & 1.57586800 & -3.54928500 & -2.48908800 \\
\hline $\mathrm{H}$ & 2.07963200 & -5.10515200 & -1.83088800 \\
\hline $\mathrm{H}$ & 0.80318000 & -5.04000800 & -3.05935800 \\
\hline $\mathrm{C}$ & -3.60320100 & -5.01575500 & -0.76290000 \\
\hline $\mathrm{H}$ & -4.03573200 & -4.29035100 & -1.46470900 \\
\hline $\mathrm{H}$ & -3.66464300 & -6.00062500 & -1.24186400 \\
\hline $\mathrm{H}$ & -4.23751300 & -5.03981300 & 0.12993700 \\
\hline $\mathrm{C}$ & 5.98692900 & -2.66614900 & 1.74877000 \\
\hline $\mathrm{H}$ & 6.74976500 & -3.28157600 & 1.25568400 \\
\hline $\mathrm{H}$ & 6.25424800 & -1.61884100 & 1.59101200 \\
\hline $\mathrm{H}$ & 6.05347800 & -2.87652300 & 2.82206600 \\
\hline $\mathrm{C}$ & 6.36778700 & -2.39922300 & -1.44171800 \\
\hline $\mathrm{H}$ & 6.65580000 & -2.39698700 & -2.49919500 \\
\hline $\mathrm{H}$ & 7.28188200 & -2.58418900 & -0.86362400 \\
\hline $\mathrm{H}$ & 5.69328300 & -3.24236300 & -1.27735900 \\
\hline $\mathrm{C}$ & -0.34001400 & 0.39829500 & 3.99286900 \\
\hline $\mathrm{O}$ & -1.64971800 & 0.11024600 & 3.47824600 \\
\hline $\mathrm{C}$ & -2.55885000 & 0.88663200 & 4.27040300 \\
\hline $\mathrm{C}$ & -1.85819300 & 2.24210200 & 4.48208300 \\
\hline $\mathrm{C}$ & -0.34941900 & 1.90646500 & 4.31317300 \\
\hline $\mathrm{H}$ & -0.17257300 & -0.19816200 & 4.90321600 \\
\hline $\mathrm{H}$ & 0.37980100 & 0.10917100 & 3.22689800 \\
\hline $\mathrm{H}$ & -3.50434900 & 0.94831600 & 3.72852800 \\
\hline $\mathrm{H}$ & -2.72540500 & 0.37737400 & 5.23322800 \\
\hline & -2.18147700 & 2.96326600 & 3.7249060 \\
\hline
\end{tabular}


Supporting Information

$\begin{array}{llll}\mathrm{H} & -2.09028600 & 2.66760000 & 5.46346100 \\ \mathrm{H} & 0.08569100 & 2.47622500 & 3.48694600 \\ \mathrm{H} & 0.23343100 & 2.12953100 & 5.21229700\end{array}$

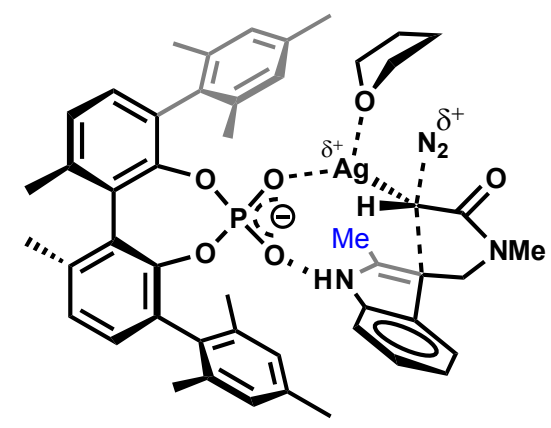

TS $_{\text {MAJOR-Me }}$

\section{$\mathrm{TS}_{\text {MAJOR-Me }}$}

E(RB3LYP) : -3057.297516 Hartree

Zero-point correction $=$ 0.937520 (Hartree/Particle)

Thermal correction to Energy= 1.000921

Thermal correction to Enthalpy= 1.001865

Thermal correction to Gibbs Free Energy= 0.832054

Sum of electronic and zero-point Energies= $-3056.359996$

Sum of electronic and thermal Energies= $-3056.296595$

Sum of electronic and thermal Enthalpies= $-3056.295651$

Sum of electronic and thermal Free Energies= $-3056.465462$

Cartesian Coordinates

Atom $\mathrm{X}$ $\mathrm{Y}$

Z

$\begin{array}{llrr}\mathrm{C} & 4.33749400 & 1.31998600 & -0.80256100 \\ \mathrm{C} & 5.53438300 & 1.20936100 & -1.52350100 \\ \mathrm{C} & 6.09232300 & -0.03317600 & -1.80077100 \\ \mathrm{C} & 5.51859500 & -1.22005800 & -1.32541000 \\ \mathrm{C} & 4.35372400 & -1.13917100 & -0.52940200 \\ \mathrm{C} & 3.76331400 & 0.12872400 & -0.34200200 \\ \mathrm{H} & 6.01371600 & 2.11418700 & -1.88765000 \\ \mathrm{C} & 3.72308600 & -2.33983000 & 0.10314100\end{array}$




\begin{tabular}{|c|c|c|c|}
\hline $\mathrm{C}$ & 2.36425600 & -2.62072700 & -0.14977600 \\
\hline $\mathrm{C}$ & 4.42805900 & -3.19417200 & 0.98148700 \\
\hline $\mathrm{C}$ & 1.70636300 & -3.75289400 & 0.34823700 \\
\hline $\mathrm{C}$ & 3.77826700 & -4.33191800 & 1.47866800 \\
\hline $\mathrm{C}$ & 2.45752400 & -4.61917900 & 1.15384000 \\
\hline $\mathrm{H}$ & 1.98389700 & -5.51501200 & 1.54656400 \\
\hline $\mathrm{O}$ & 1.65621900 & -1.75394200 & -0.97108400 \\
\hline $\mathrm{O}$ & 2.57226300 & 0.20173300 & 0.37137200 \\
\hline $\mathrm{P}$ & 1.19133300 & -0.28897300 & -0.38088400 \\
\hline $\mathrm{Ag}$ & -1.92986700 & -0.13780000 & 0.7374020 \\
\hline $\mathrm{O}$ & 0.20885600 & -0.45802400 & 0.77012400 \\
\hline $\mathrm{O}$ & 0.83888800 & 0.54804400 & -1.57476300 \\
\hline $\mathrm{H}$ & 6.99347400 & -0.09139100 & -2.40708000 \\
\hline $\mathrm{H}$ & 4.32141600 & -4.99721400 & 2.14607000 \\
\hline $\mathrm{C}$ & -5.01374300 & -0.75170300 & -0.12133100 \\
\hline $\mathrm{O}$ & -5.36814300 & -1.73876100 & 0.52942400 \\
\hline $\mathrm{N}$ & -5.42554600 & -0.53046700 & -1.40769600 \\
\hline $\mathrm{C}$ & -6.38971300 & -1.45544700 & -1.99409800 \\
\hline $\mathrm{H}$ & -7.38074000 & -0.98771600 & -2.07346600 \\
\hline $\mathrm{H}$ & -6.06170600 & -1.74921900 & -2.99768700 \\
\hline $\mathrm{H}$ & -6.46328500 & -2.33418300 & -1.35606200 \\
\hline $\mathrm{C}$ & -5.19257600 & 0.72159200 & -2.15293800 \\
\hline $\mathrm{H}$ & -5.31384700 & 0.45954000 & -3.21217200 \\
\hline $\mathrm{H}$ & -5.99257500 & 1.44171700 & -1.92391300 \\
\hline $\mathrm{C}$ & -3.85602100 & 1.34810200 & -1.90781500 \\
\hline $\mathrm{C}$ & -2.61653100 & 0.79019400 & -2.23749700 \\
\hline $\mathrm{C}$ & -3.59518200 & 2.69378000 & -1.44917800 \\
\hline $\mathrm{N}$ & -1.62968100 & 1.69465000 & -1.96783300 \\
\hline $\mathrm{C}$ & -2.18357000 & 2.85979100 & -1.46908000 \\
\hline $\mathrm{H}$ & -0.63276000 & 1.42404800 & -1.91751300 \\
\hline $\mathrm{C}$ & -4.39736200 & 3.75445900 & -0.99281400 \\
\hline $\mathrm{H}$ & -5.48181600 & 3.67012500 & -0.97922000 \\
\hline $\mathrm{C}$ & -3.78210800 & 4.92782400 & -0.56633400 \\
\hline $\mathrm{H}$ & -4.39308500 & 5.75613600 & -0.21776900 \\
\hline
\end{tabular}


Supporting Information

\begin{tabular}{|c|c|c|c|}
\hline $\mathrm{C}$ & -2.37931200 & 5.06459100 & -0.58300400 \\
\hline $\mathrm{H}$ & -1.92961900 & 5.99324900 & -0.24271700 \\
\hline $\mathrm{C}$ & -1.55959700 & 4.03317300 & -1.03415100 \\
\hline $\mathrm{H}$ & -0.47758600 & 4.13209000 & -1.05205800 \\
\hline $\mathrm{C}$ & 5.83957100 & -2.91322300 & 1.45013100 \\
\hline $\mathrm{H}$ & 6.57578200 & -3.53445600 & 0.92488700 \\
\hline $\mathrm{H}$ & 6.12262800 & -1.86849300 & 1.30247400 \\
\hline $\mathrm{H}$ & 5.93271500 & -3.14314500 & 2.51740700 \\
\hline $\mathrm{C}$ & 6.14937100 & -2.53428300 & -1.73264500 \\
\hline $\mathrm{H}$ & 6.41934400 & -2.50621900 & -2.79439300 \\
\hline $\mathrm{H}$ & 7.07258900 & -2.73424500 & -1.17446600 \\
\hline $\mathrm{H}$ & 5.47648200 & -3.38053500 & -1.57745700 \\
\hline $\mathrm{C}$ & 0.26242800 & -4.02409100 & 0.06058600 \\
\hline $\mathrm{C}$ & -0.14214500 & -4.45337500 & -1.21949400 \\
\hline $\mathrm{C}$ & -0.69990000 & -3.86288000 & 1.08315100 \\
\hline $\mathrm{C}$ & -1.49804300 & -4.71304600 & -1.45608100 \\
\hline $\mathrm{C}$ & -2.04454700 & -4.12361400 & 0.80015000 \\
\hline $\mathrm{C}$ & -2.46689100 & -4.55458100 & -0.46284100 \\
\hline $\mathrm{H}$ & -1.80115100 & -5.05287300 & -2.44499500 \\
\hline $\mathrm{H}$ & -2.78424200 & -3.98845400 & 1.58719500 \\
\hline $\mathrm{C}$ & 3.72439200 & 2.66108800 & -0.53985700 \\
\hline $\mathrm{C}$ & 3.80381900 & 3.23110400 & 0.74914900 \\
\hline $\mathrm{C}$ & 3.10166900 & 3.37502500 & -1.58576800 \\
\hline $\mathrm{C}$ & 3.26526600 & 4.50348800 & 0.96875500 \\
\hline $\mathrm{C}$ & 2.57674600 & 4.64669000 & -1.32106000 \\
\hline $\mathrm{C}$ & 2.65057200 & 5.23219400 & -0.05422100 \\
\hline $\mathrm{H}$ & 3.33972700 & 4.94025000 & 1.96336800 \\
\hline $\mathrm{H}$ & 2.10173200 & 5.19501600 & -2.13293500 \\
\hline $\mathrm{C}$ & -3.92434600 & -4.85943800 & -0.72320200 \\
\hline $\mathrm{H}$ & -4.13015500 & -4.93622500 & -1.79667400 \\
\hline $\mathrm{H}$ & -4.21841600 & -5.81409300 & -0.26599900 \\
\hline $\mathrm{H}$ & -4.57185000 & -4.08320400 & -0.30017100 \\
\hline $\mathrm{C}$ & 0.85797600 & -4.63469900 & -2.33917100 \\
\hline $\mathrm{H}$ & 1.26044600 & -3.66933100 & -2.66780600 \\
\hline
\end{tabular}


Supporting Information

\begin{tabular}{|c|c|c|c|}
\hline $\mathrm{H}$ & 1.71011000 & -5.24877800 & -2.02564300 \\
\hline $\mathrm{H}$ & 0.39182600 & -5.11845800 & -3.20367300 \\
\hline $\mathrm{C}$ & 4.46094700 & 2.49242300 & 1.89356500 \\
\hline $\mathrm{H}$ & 3.87911900 & 1.60825800 & 2.17917800 \\
\hline $\mathrm{H}$ & 5.46520900 & 2.14367700 & 1.62640600 \\
\hline $\mathrm{H}$ & 4.54944300 & 3.13725200 & 2.77390900 \\
\hline C & 2.96020600 & 2.78520200 & -2.97152200 \\
\hline $\mathrm{H}$ & 2.44283100 & 3.48439000 & -3.63686500 \\
\hline $\mathrm{H}$ & 3.93160200 & 2.55243600 & -3.42209800 \\
\hline $\mathrm{H}$ & 2.38408200 & 1.85435100 & -2.93300600 \\
\hline $\mathrm{C}$ & 2.11840700 & 6.62528600 & 0.19494900 \\
\hline $\mathrm{H}$ & 1.33698300 & 6.89156900 & -0.52526700 \\
\hline $\mathrm{H}$ & 1.69859800 & 6.72176800 & 1.20267300 \\
\hline $\mathrm{H}$ & 2.91303000 & 7.37838800 & 0.10371600 \\
\hline $\mathrm{C}$ & -0.30986200 & -3.38882600 & 2.46683500 \\
\hline $\mathrm{H}$ & 0.22896100 & -2.43683800 & 2.41267600 \\
\hline $\mathrm{H}$ & -1.20047300 & -3.24875000 & 3.08931000 \\
\hline $\mathrm{H}$ & 0.34300200 & -4.10420900 & 2.98028400 \\
\hline $\mathrm{C}$ & -4.03483500 & 0.23735600 & 0.47798200 \\
\hline $\mathrm{H}$ & -4.34154600 & 1.28022000 & 0.51705000 \\
\hline $\mathrm{N}$ & -4.55058300 & -0.03131500 & 2.25230600 \\
\hline $\mathrm{N}$ & -4.53385600 & -0.12076700 & 3.35585100 \\
\hline $\mathrm{C}$ & -0.65851900 & 0.72100000 & 4.01857400 \\
\hline $\mathrm{O}$ & -1.66801500 & 1.27142300 & 3.15767400 \\
\hline $\mathrm{C}$ & -1.36709100 & 2.66667700 & 2.93337500 \\
\hline $\mathrm{C}$ & 0.08282700 & 2.90275700 & 3.41563600 \\
\hline $\mathrm{C}$ & 0.61681200 & 1.47707100 & 3.65519000 \\
\hline $\mathrm{H}$ & -0.93882100 & 0.87950000 & 5.07260300 \\
\hline $\mathrm{H}$ & -0.60923800 & -0.35293200 & 3.82227400 \\
\hline $\mathrm{H}$ & -1.49527700 & 2.86215200 & 1.86425700 \\
\hline $\mathrm{H}$ & -2.08760400 & 3.28509400 & 3.48535200 \\
\hline $\mathrm{H}$ & 0.67882600 & 3.45101400 & 2.68032400 \\
\hline $\mathrm{H}$ & 0.08673300 & 3.47918400 & 4.34789300 \\
\hline & 1.03573000 & 1.05823400 & 2.73604100 \\
\hline
\end{tabular}




$\begin{array}{lrrr}\mathrm{H} & 1.37588800 & 1.43374600 & 4.44290600 \\ \mathrm{C} & -2.29115500 & -0.54083400 & -2.84138300 \\ \mathrm{H} & -2.55015200 & -0.55478500 & -3.90916700 \\ \mathrm{H} & -1.22242800 & -0.75266700 & -2.75198700 \\ \mathrm{H} & -2.84873100 & -1.34812400 & -2.35686400\end{array}$

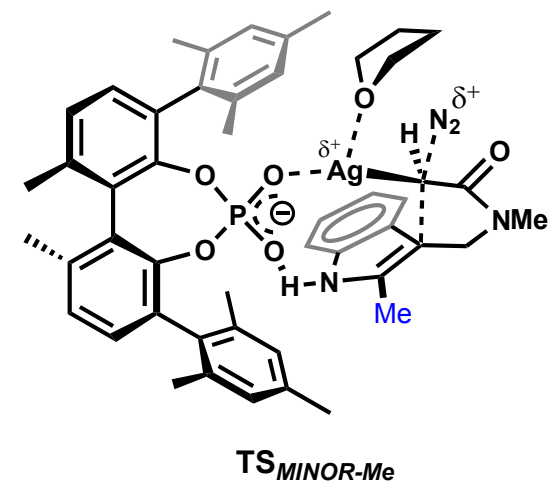

$\mathrm{TS}_{M I N O R-M e}$

E(RB3LYP) : -3057.295353 Hartree

Zero-point correction $=$ 0.937477 (Hartree/Particle)

Thermal correction to Energy= 1.000960

Thermal correction to Enthalpy= 1.001904

Thermal correction to Gibbs Free Energy=

0.830039

Sum of electronic and zero-point Energies= $-3056.357876$

Sum of electronic and thermal Energies= $-3056.294394$

Sum of electronic and thermal Enthalpies= $-3056.293450$

Sum of electronic and thermal Free Energies= $-3056.465314$

\section{Cartesian Coordinates}

\begin{tabular}{lrrr} 
Atom & X & Y & $Z$ \\
\hdashline-1.5 & & & \\
O & -5.01942100 & 1.93346600 & 1.93213900 \\
$\mathrm{~N}$ & -4.58172100 & -1.14415000 & 3.10334600 \\
$\mathrm{~N}$ & -4.55494300 & -0.54781400 & 2.16985900 \\
$\mathrm{~N}$ & -1.87997400 & 0.14593200 & -2.47100100 \\
$\mathrm{~N}$ & -5.16256300 & 2.23723400 & -0.32251400 \\
$\mathrm{C}$ & -2.35871500 & -2.23245900 & -3.15306000
\end{tabular}


Supporting Information

\begin{tabular}{|c|c|c|c|}
\hline $\mathrm{C}$ & -3.40066500 & -3.13483900 & -3.34816800 \\
\hline $\mathrm{C}$ & -4.74111200 & -2.76213000 & -3.12132200 \\
\hline $\mathrm{C}$ & -5.06853100 & -1.48116300 & -2.68668900 \\
\hline $\mathrm{C}$ & -2.69272200 & -0.94375000 & -2.72867500 \\
\hline $\mathrm{C}$ & -4.03579300 & -0.54986100 & -2.48025700 \\
\hline $\mathrm{C}$ & -2.63748000 & 1.19824600 & -2.04456700 \\
\hline $\mathrm{C}$ & -3.98122000 & 0.81392600 & -2.00563700 \\
\hline $\mathrm{C}$ & -5.14115600 & 1.70714300 & -1.70070700 \\
\hline $\mathrm{C}$ & -5.91050800 & 3.47549300 & -0.12504600 \\
\hline $\mathrm{C}$ & -4.79538100 & 1.52719100 & 0.78661100 \\
\hline $\mathrm{C}$ & -4.02222600 & 0.24032100 & 0.58329200 \\
\hline $\mathrm{H}$ & -1.32434800 & -2.51704900 & -3.32003100 \\
\hline $\mathrm{H}$ & -3.17660500 & -4.14258900 & -3.68614600 \\
\hline $\mathrm{H}$ & -5.53160700 & -3.48758400 & -3.29374100 \\
\hline $\mathrm{H}$ & -6.10882600 & -1.20881300 & -2.52226200 \\
\hline $\mathrm{H}$ & -0.85167100 & 0.13461000 & -2.36287400 \\
\hline $\mathrm{H}$ & -5.13890500 & 2.58962000 & -2.35355000 \\
\hline $\mathrm{H}$ & -6.08495800 & 1.17972000 & -1.90446000 \\
\hline $\mathrm{H}$ & -5.52339700 & 4.24977000 & -0.79647500 \\
\hline $\mathrm{H}$ & -6.97827500 & 3.32917700 & -0.33880000 \\
\hline $\mathrm{H}$ & -5.79874900 & 3.79007600 & 0.91113000 \\
\hline $\mathrm{H}$ & -4.49049500 & -0.54114800 & -0.01018900 \\
\hline $\mathrm{Ag}$ & -1.88368000 & 0.09079300 & 0.89363400 \\
\hline $\mathrm{C}$ & 2.31100700 & -3.35173000 & 0.88897400 \\
\hline $\mathrm{C}$ & 3.17871800 & -3.93136700 & 1.82427700 \\
\hline $\mathrm{C}$ & 4.41152700 & -3.35678300 & 2.11140300 \\
\hline $\mathrm{C}$ & 4.86238400 & -2.21074600 & 1.44290400 \\
\hline $\mathrm{C}$ & 4.04372900 & -1.64437400 & 0.43920600 \\
\hline $\mathrm{C}$ & 2.76367800 & -2.19977300 & 0.23285100 \\
\hline $\mathrm{H}$ & 2.86475400 & -4.83267500 & 2.34403200 \\
\hline $\mathrm{C}$ & 4.47747700 & -0.47172000 & -0.38296000 \\
\hline $\mathrm{C}$ & 3.67728500 & 0.68950000 & -0.40541500 \\
\hline
\end{tabular}


Supporting Information

\begin{tabular}{|c|c|c|c|}
\hline $\mathrm{C}$ & 5.65964000 & -0.48076600 & -1.15762900 \\
\hline $\mathrm{C}$ & 4.05654600 & 1.87221400 & -1.05202400 \\
\hline $\mathrm{C}$ & 6.03676600 & 0.69342700 & -1.82302900 \\
\hline $\mathrm{C}$ & 5.27190200 & 1.85195500 & -1.74945200 \\
\hline $\mathrm{H}$ & 5.60188800 & 2.75492600 & -2.25629800 \\
\hline $\mathrm{O}$ & 2.47331700 & 0.67277900 & 0.28834100 \\
\hline $\mathrm{O}$ & 1.93205700 & -1.60696300 & -0.70722500 \\
\hline $\mathrm{P}$ & 1.21244300 & -0.16710200 & -0.35939100 \\
\hline $\mathrm{O}$ & 0.74364100 & 0.39805800 & -1.66862100 \\
\hline $\mathrm{O}$ & 0.25083000 & -0.31505200 & 0.81041700 \\
\hline $\mathrm{H}$ & 5.04278400 & -3.80024300 & 2.87816900 \\
\hline $\mathrm{H}$ & 6.94951200 & 0.69188300 & -2.41462300 \\
\hline $\mathrm{C}$ & 3.22313900 & 3.11512100 & -0.99728600 \\
\hline $\mathrm{C}$ & 3.18972900 & 3.88485200 & 0.18558900 \\
\hline $\mathrm{C}$ & 2.49835700 & 3.53841500 & -2.13136200 \\
\hline $\mathrm{C}$ & 2.43533000 & 5.06241100 & 0.21383200 \\
\hline $\mathrm{C}$ & 1.75665300 & 4.72418100 & -2.05799800 \\
\hline $\mathrm{C}$ & 1.71285200 & 5.50303000 & -0.89925000 \\
\hline $\mathrm{H}$ & 2.42007700 & 5.65434600 & 1.12753700 \\
\hline $\mathrm{H}$ & 1.19770000 & 5.04543100 & -2.93529500 \\
\hline $\mathrm{C}$ & 0.96719500 & -3.95062900 & 0.61196500 \\
\hline $\mathrm{C}$ & 0.73880900 & -4.64500200 & -0.59605400 \\
\hline $\mathrm{C}$ & -0.06341900 & -3.85286800 & 1.57004300 \\
\hline $\mathrm{C}$ & -0.51322100 & -5.22309700 & -0.82476100 \\
\hline $\mathrm{C}$ & -1.30301400 & -4.44521700 & 1.29739300 \\
\hline $\mathrm{C}$ & -1.55265600 & -5.12976900 & 0.10581600 \\
\hline $\mathrm{H}$ & -0.67866800 & -5.76449200 & -1.75485000 \\
\hline $\mathrm{H}$ & -2.09335400 & -4.37109500 & 2.04279800 \\
\hline $\mathrm{C}$ & 2.47452400 & 2.72190300 & -3.40442800 \\
\hline $\mathrm{H}$ & 1.87755100 & 3.22392400 & -4.17320800 \\
\hline $\mathrm{H}$ & 3.47901500 & 2.56284500 & -3.81211000 \\
\hline $\mathrm{H}$ & 2.03594100 & 1.73593600 & -3.21517900 \\
\hline
\end{tabular}




\begin{tabular}{|c|c|c|c|}
\hline $\mathrm{C}$ & 3.95388400 & 3.45857400 & 1.41933800 \\
\hline $\mathrm{H}$ & 3.53600300 & 2.53894600 & 1.84558900 \\
\hline $\mathrm{H}$ & 5.00758400 & 3.25769800 & 1.19356800 \\
\hline & 3.91491200 & 4.23564200 & 2.18961400 \\
\hline $\mathrm{C}$ & 0.93699500 & 6.79993800 & -0.85852000 \\
\hline H & 1.58342200 & 7.66010200 & -1.08049400 \\
\hline $\mathrm{H}$ & 0.12648000 & 6.80575300 & -1.59552200 \\
\hline $\mathrm{H}$ & 0.49742000 & 6.97508600 & 0.13003700 \\
\hline $\mathrm{C}$ & 0.13320000 & -3.10258800 & 2.86938600 \\
\hline $\mathrm{F}$ & -0.79004700 & -3.10282100 & 3.45875300 \\
\hline $\mathrm{H}$ & 0.92213300 & -3.54503600 & 3.48830800 \\
\hline $\mathrm{H}$ & 0.41598200 & -2.06252300 & 2.67490000 \\
\hline $\mathrm{C}$ & 1.82260800 & -4.77471500 & -1.64280900 \\
\hline $\mathrm{H}$ & 2.04751700 & -3.80377600 & -2.09959300 \\
\hline $\mathrm{H}$ & 2.75792800 & -5.15257900 & -1.21381100 \\
\hline $\mathrm{H}$ & 1.51471300 & -5.45973600 & -2.43949000 \\
\hline C & -2.90786700 & -5.73339100 & -0.18280300 \\
\hline $\mathrm{H}$ & -3.48240500 & -5.10039000 & -0.87232300 \\
\hline $\mathrm{H}$ & -2.81710200 & -6.72115100 & -0.65011600 \\
\hline $\mathrm{H}$ & -3.49918500 & -5.84728800 & 0.73227100 \\
\hline $\mathrm{C}$ & 6.18661900 & -1.61012800 & 1.86333800 \\
\hline $\mathrm{H}$ & 7.03720000 & -2.16769300 & 1.45162200 \\
\hline H & 6.28742100 & -0.56995600 & 1.54517800 \\
\hline $\mathrm{H}$ & 6.28305400 & -1.64277400 & 2.95438300 \\
\hline $\mathrm{C}$ & 6.51438800 & -1.71603100 & -1.34328800 \\
\hline $\mathrm{H}$ & 6.80971000 & -1.81197300 & -2.39425700 \\
\hline $\mathrm{H}$ & 7.43975500 & -1.66427700 & -0.75594400 \\
\hline $\mathrm{H}$ & 5.98834600 & -2.62890200 & -1.05523600 \\
\hline C & 0.03289600 & 1.54094600 & 3.52126600 \\
\hline C & -1.34719900 & 1.23305600 & 3.25887800 \\
\hline C & -2.10163600 & 2.32201300 & 3.80560200 \\
\hline $\mathrm{C}$ & -1.30767900 & 3.57919500 & 3.4198090 \\
\hline
\end{tabular}


Supporting Information

$\begin{array}{lrrr}\mathrm{C} & 0.16058800 & 3.07376600 & 3.36073300 \\ \mathrm{H} & 0.27767400 & 1.23084600 & 4.54857700 \\ \mathrm{H} & 0.63045000 & 0.96495500 & 2.81458500 \\ \mathrm{H} & -3.10894500 & 2.27424400 & 3.38792100 \\ \mathrm{H} & -2.15761700 & 2.21353200 & 4.90114000 \\ \mathrm{H} & -1.63020200 & 3.94030800 & 2.43752700 \\ \mathrm{H} & -1.45109700 & 4.39380800 & 4.13675200 \\ \mathrm{H} & 0.62880800 & 3.33410800 & 2.40711700 \\ \mathrm{H} & 0.77557300 & 3.50283900 & 4.15830500 \\ \mathrm{C} & -2.01136200 & 2.52806700 & -1.75876500 \\ \mathrm{H} & -0.94163100 & 2.41928700 & -1.55940200 \\ \mathrm{H} & -2.12237800 & 3.20028200 & -2.62101100 \\ \mathrm{H} & -2.48829100 & 3.01335600 & -0.90171100\end{array}$




\section{Charts of ${ }^{1} \mathrm{H}^{-}$and ${ }^{13} \mathrm{C}-\mathrm{NMR}$ Spectra}

S1a

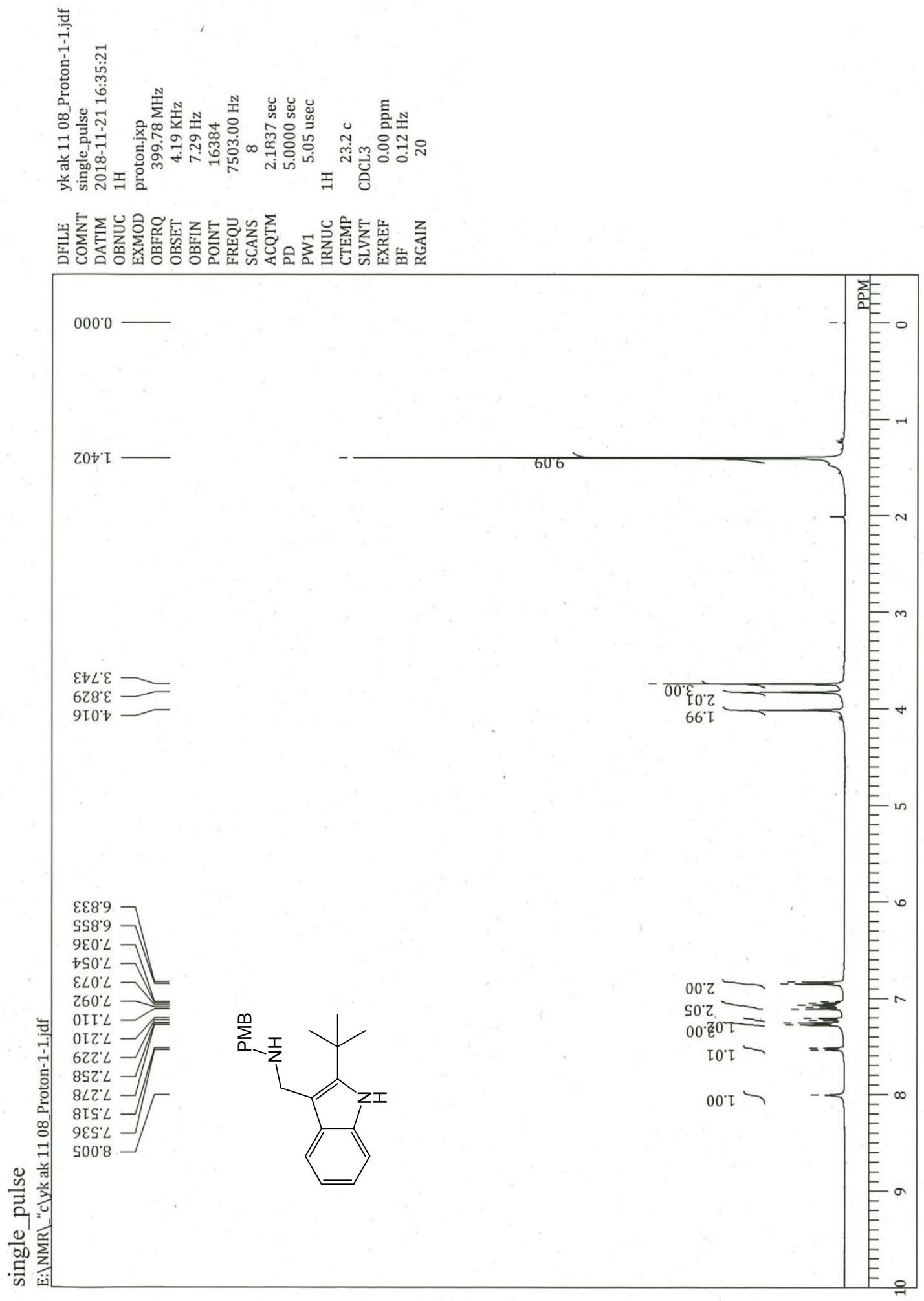


Supporting Information

S1a

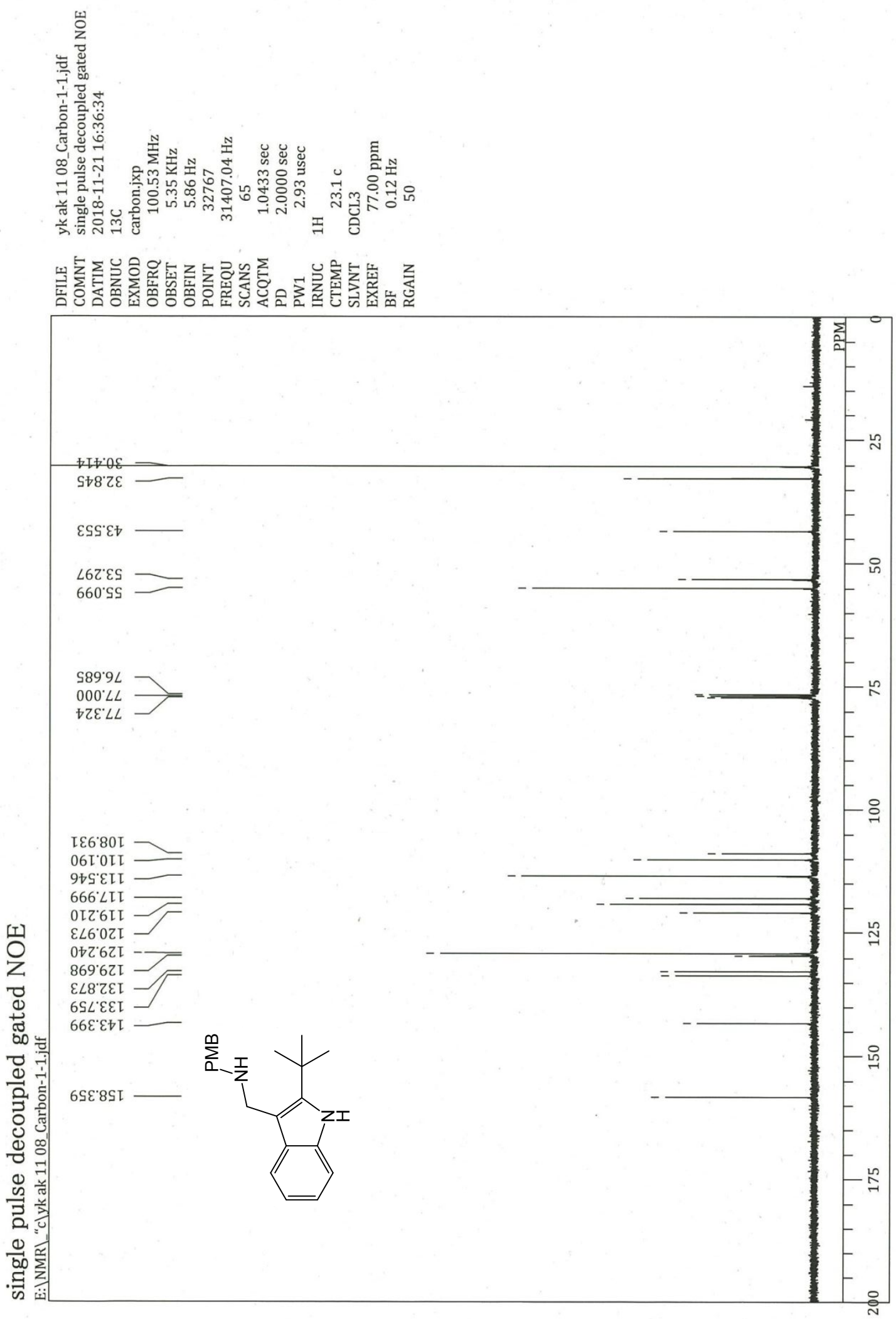


Supporting Information

S1'a
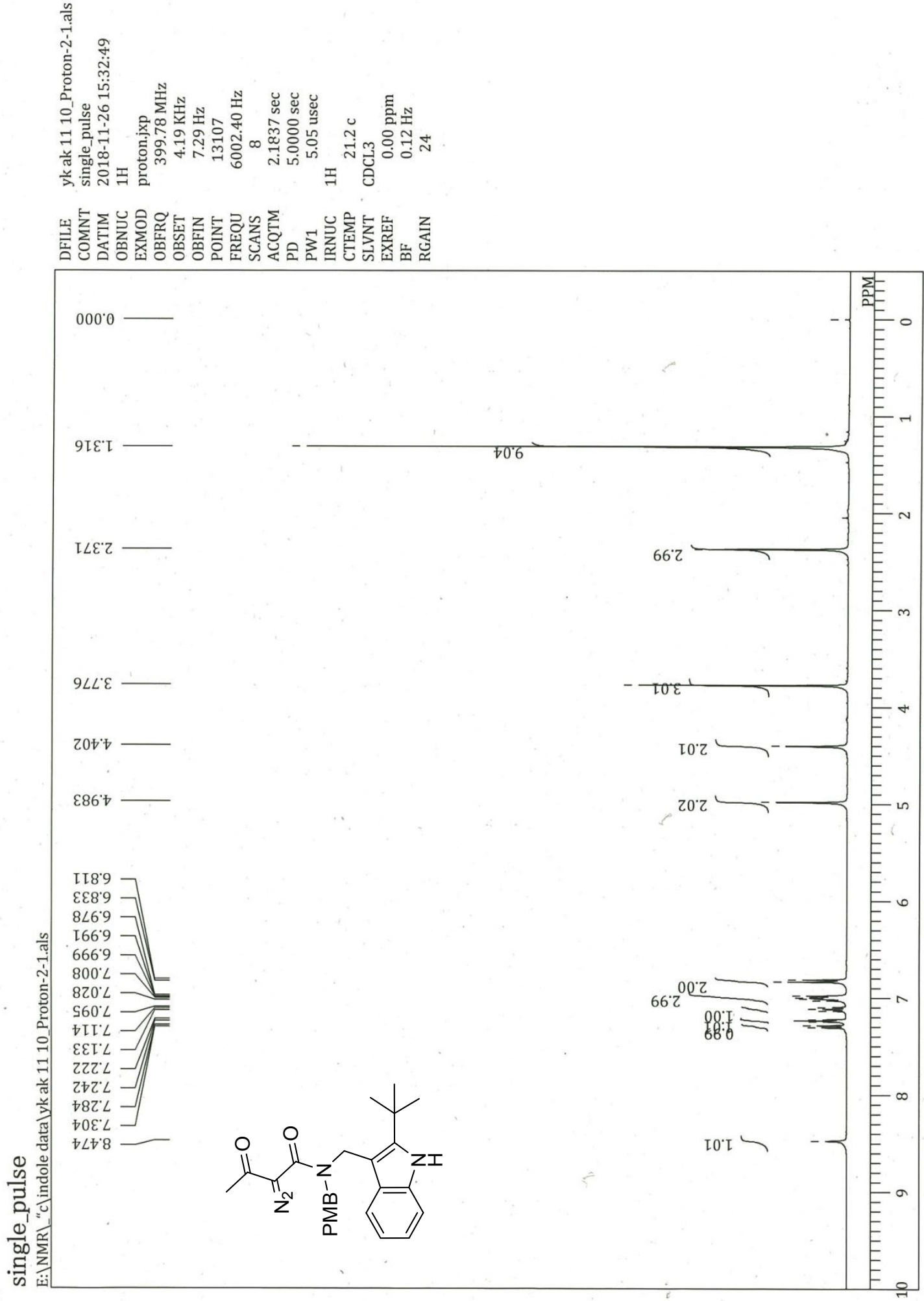
Supporting Information

S1'a

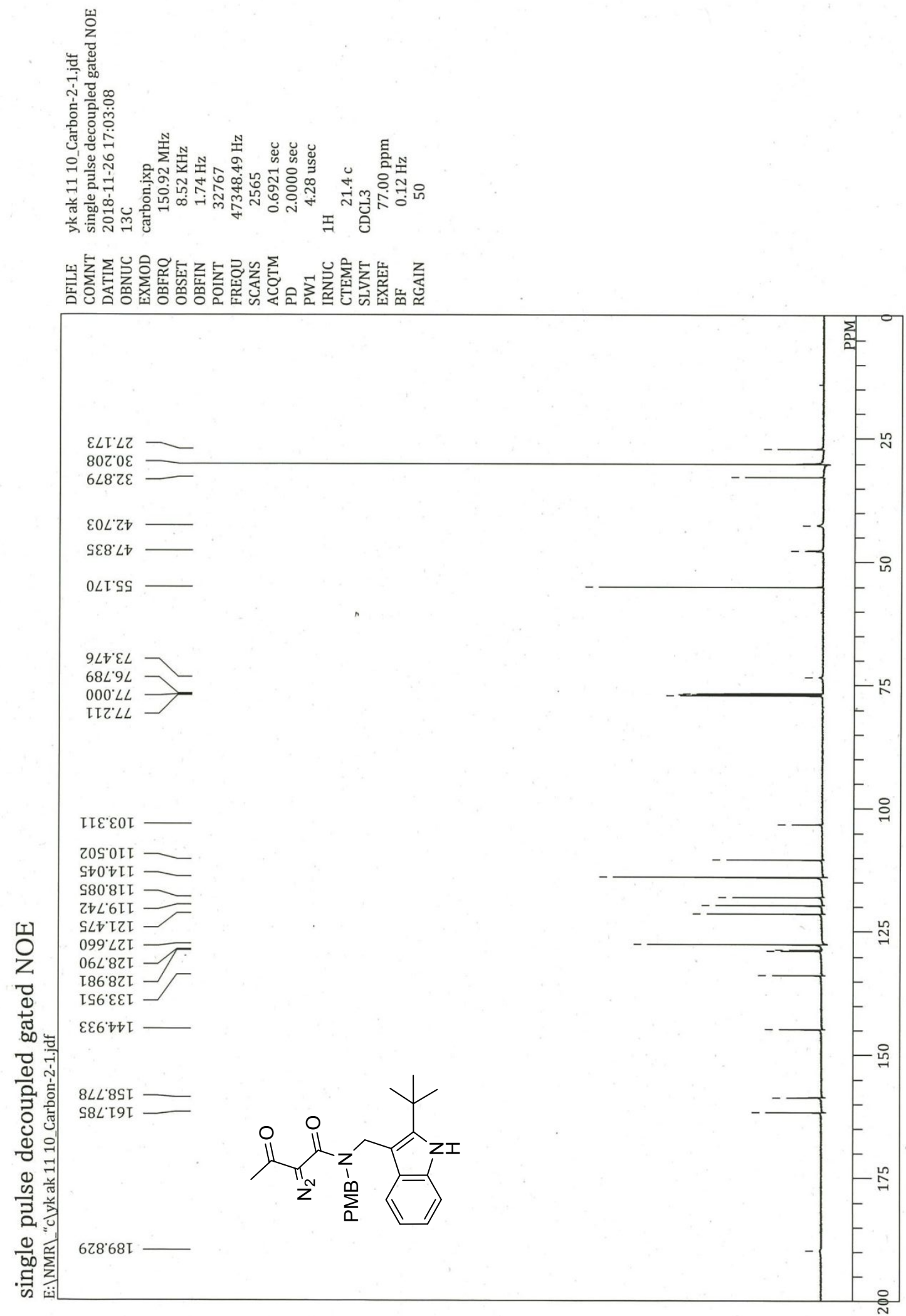


Supporting Information

S1b
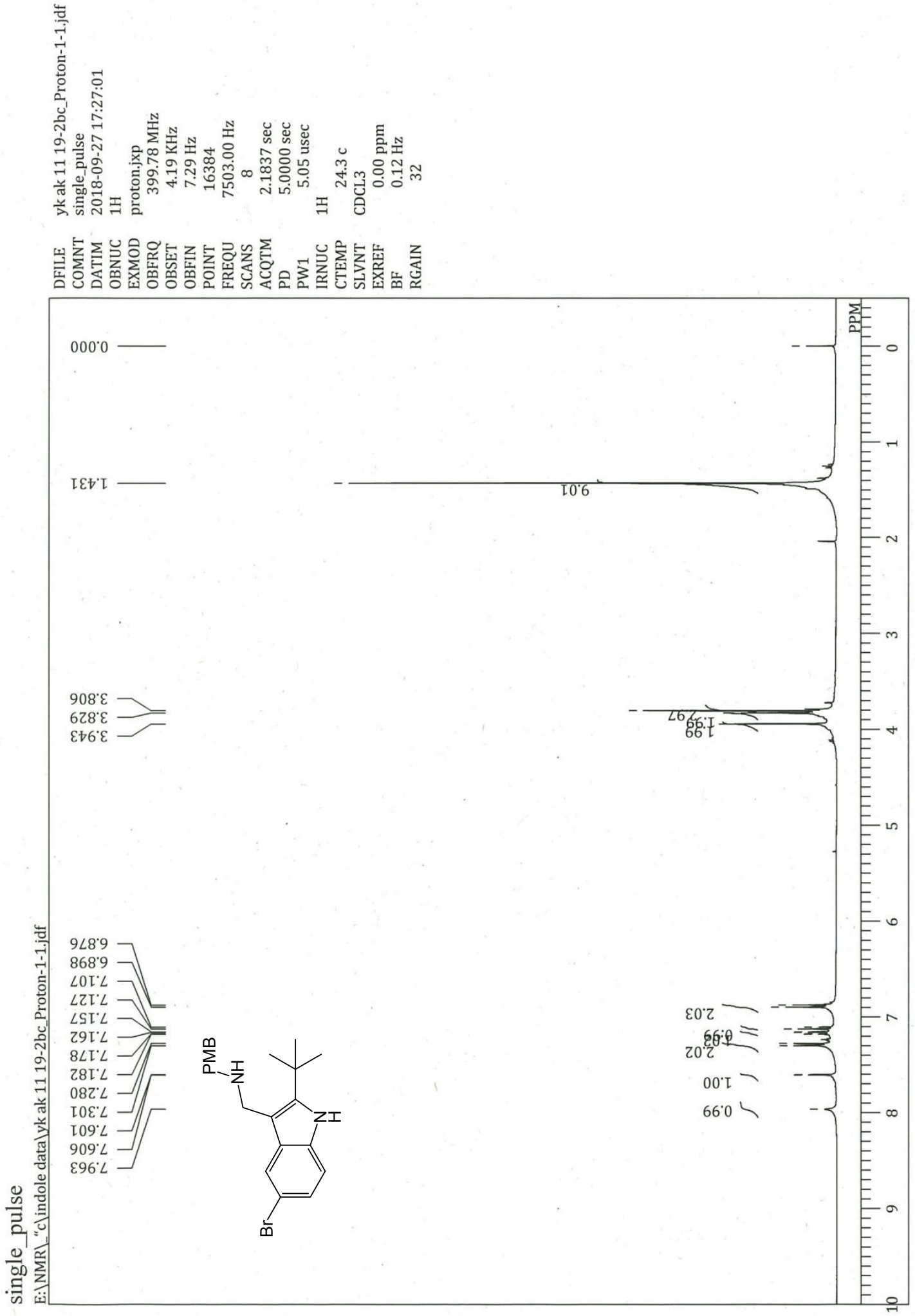
Supporting Information

S1b

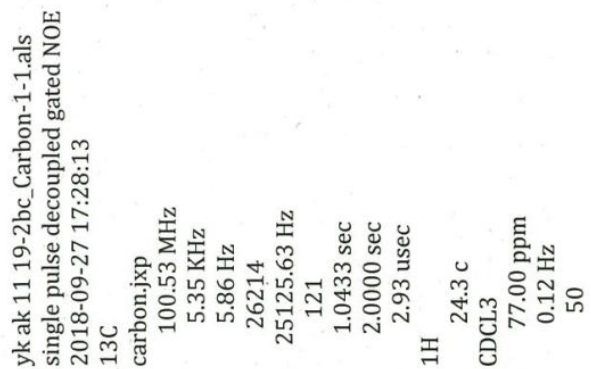

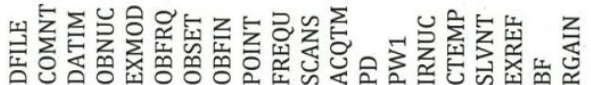

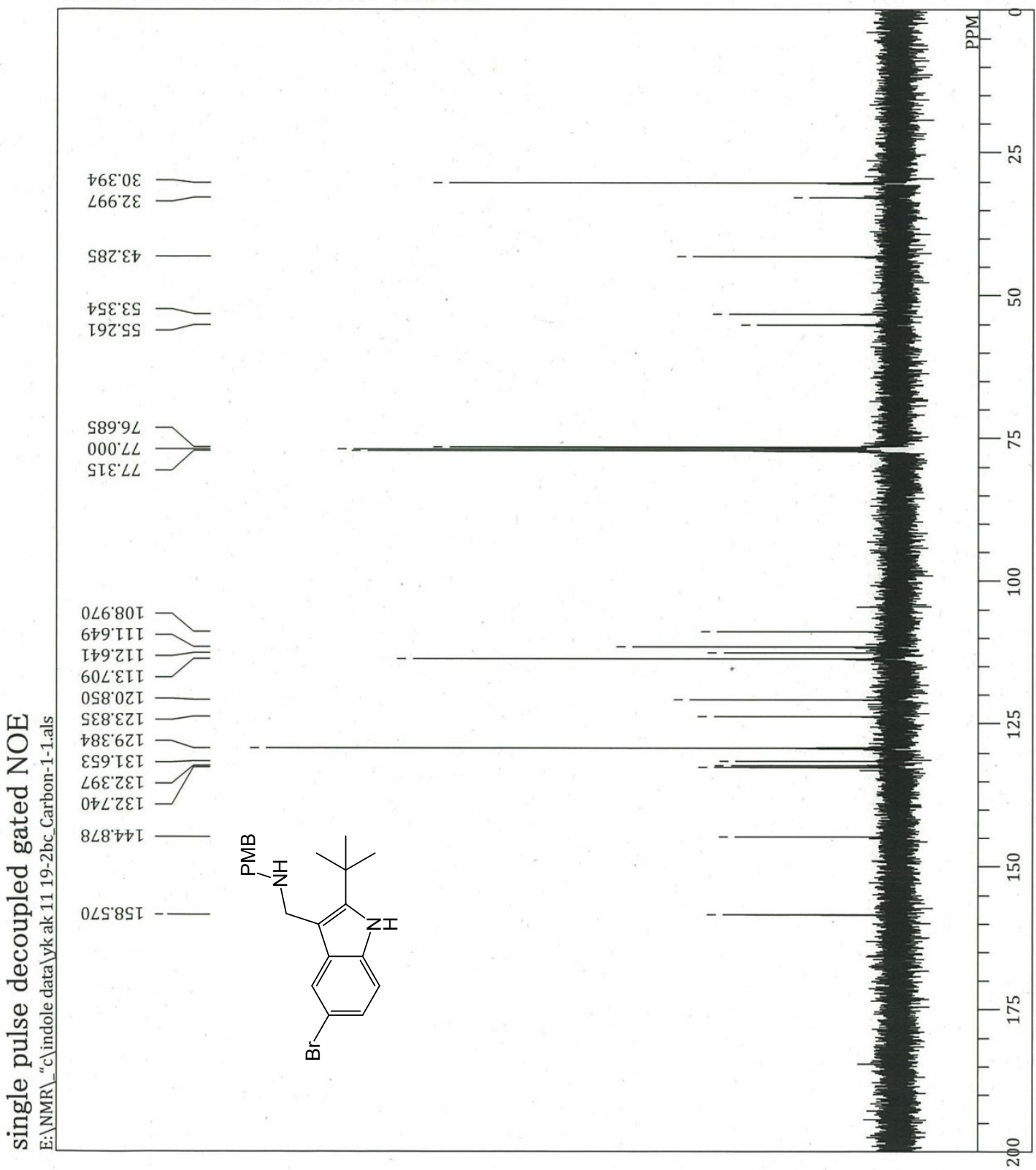


Supporting Information

S1c

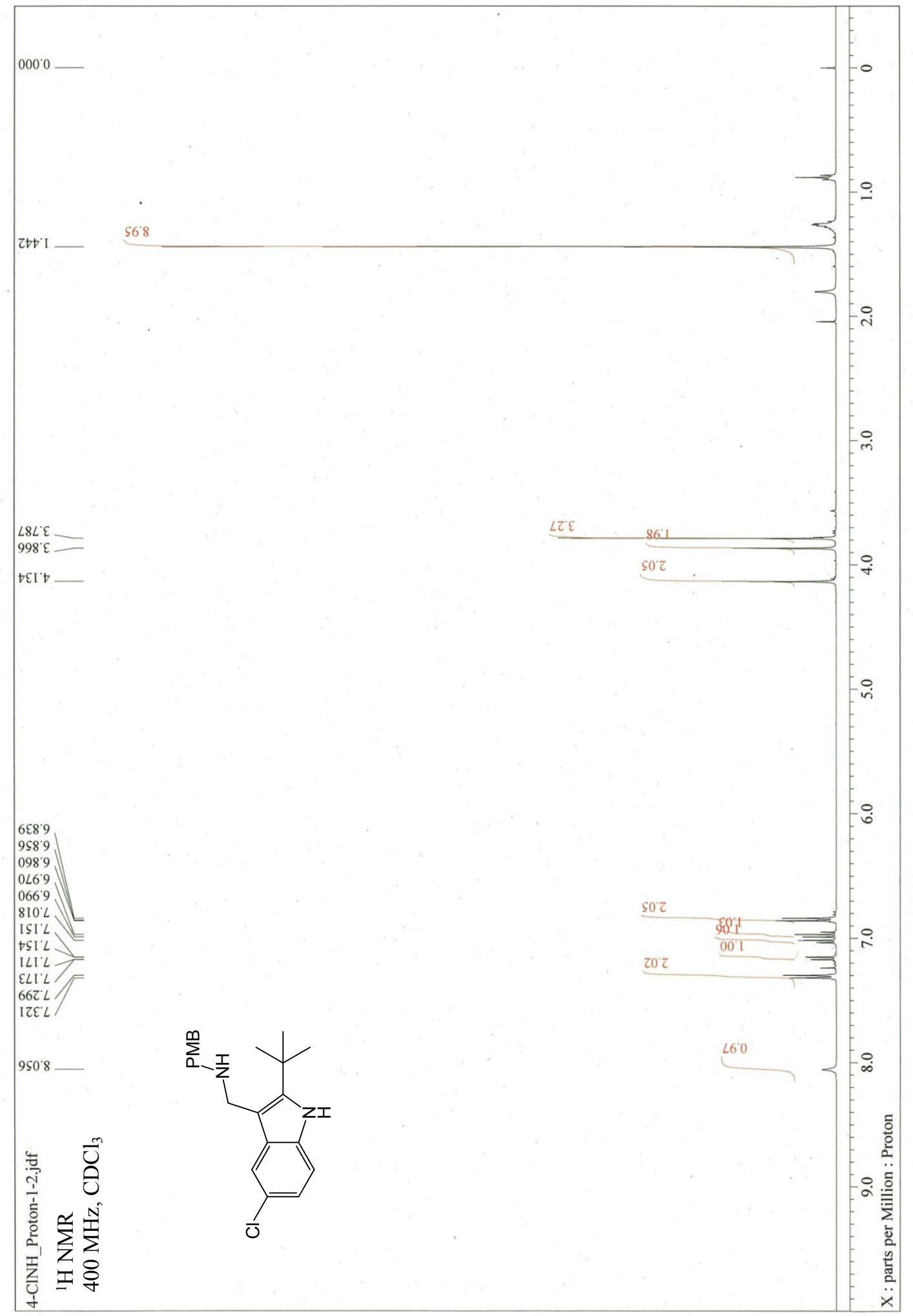


Supporting Information

S1c

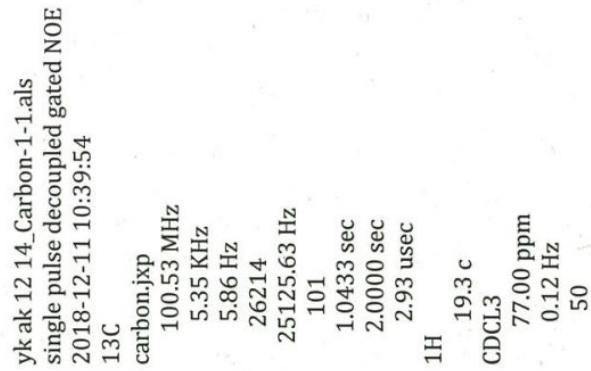

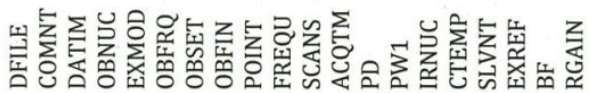

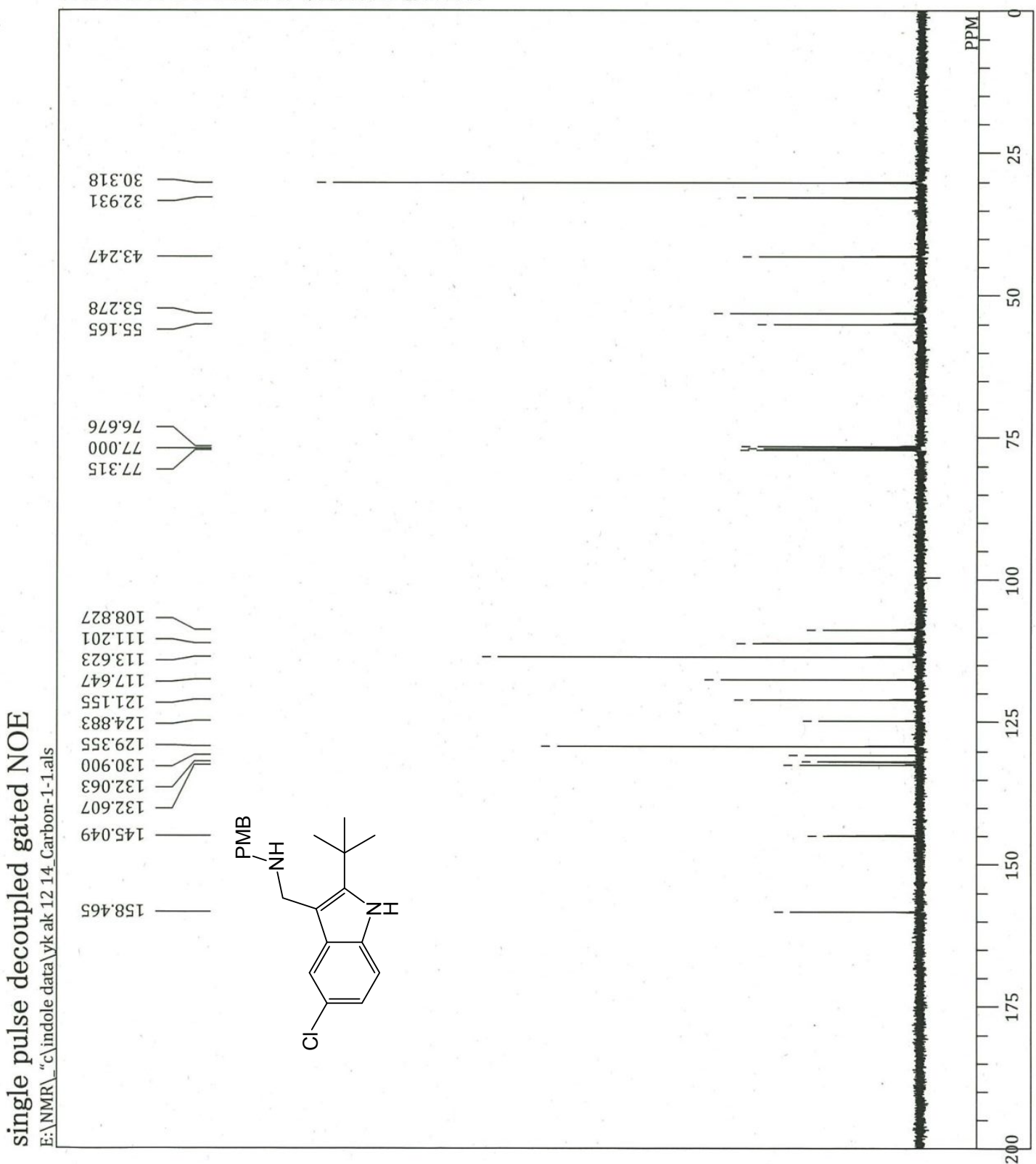


Supporting Information

S1d

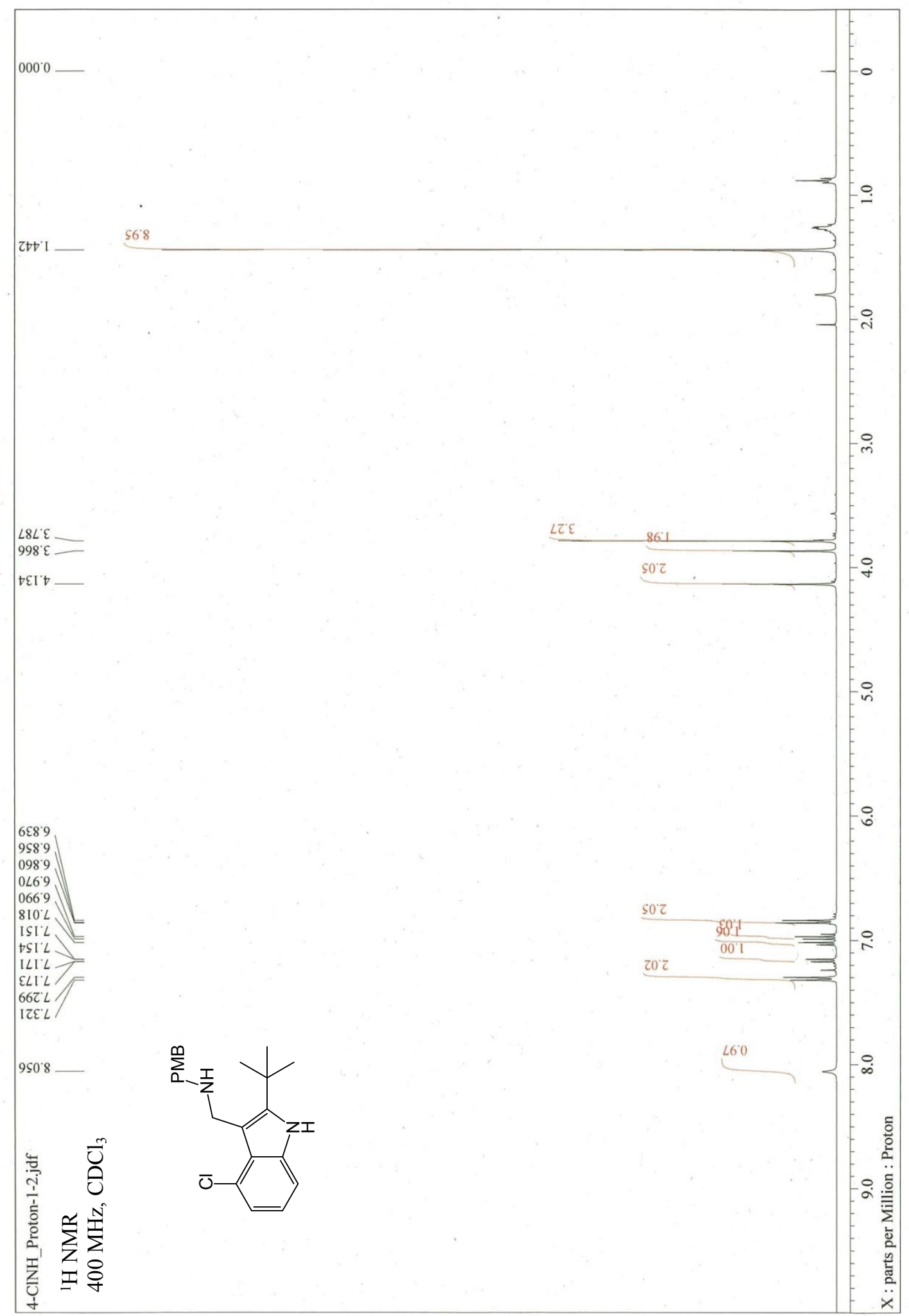


Supporting Information

S1d

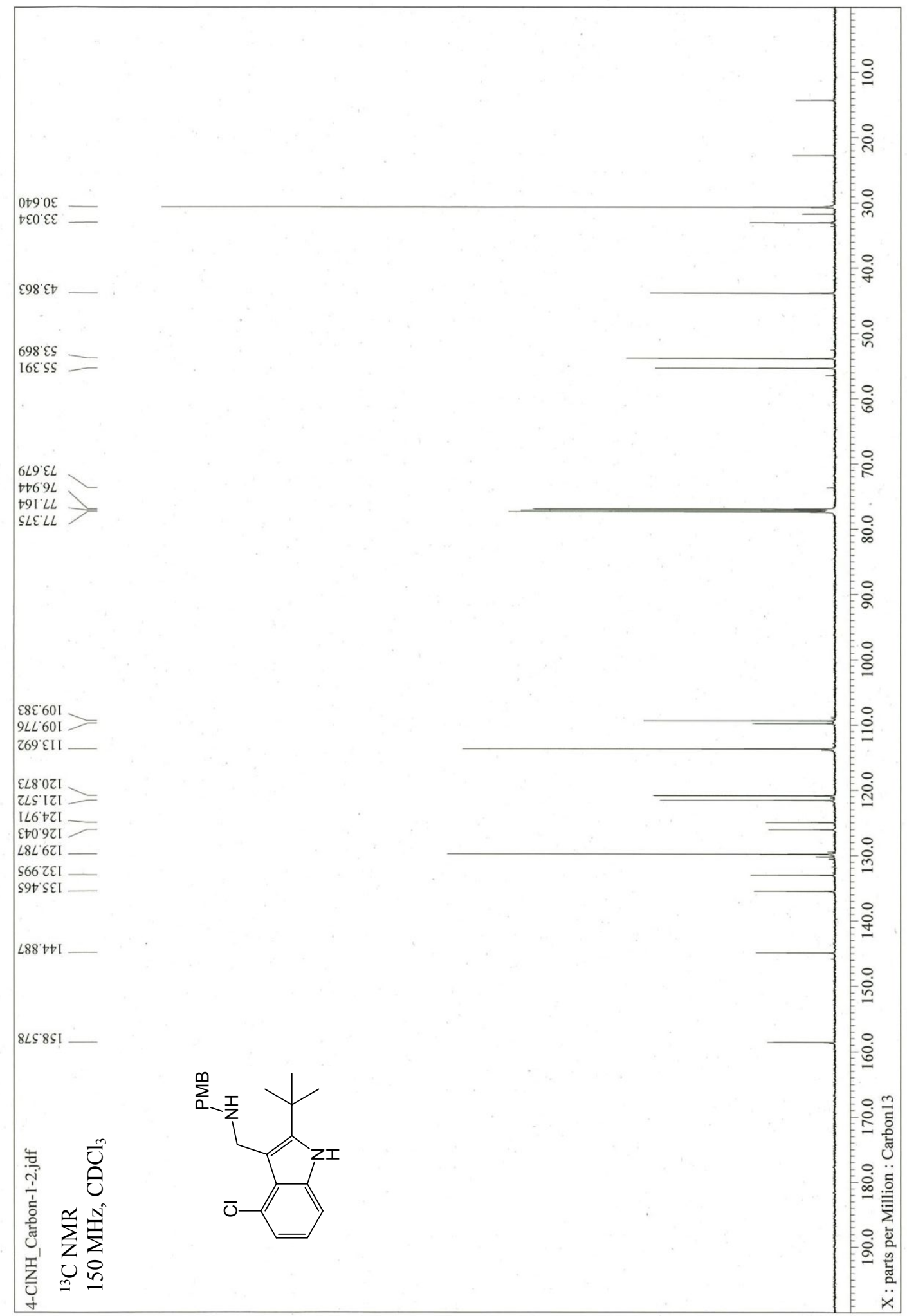


Supporting Information

S1e

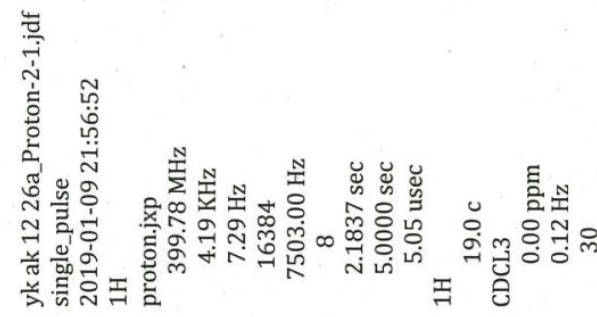

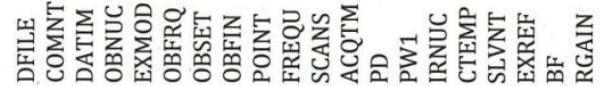

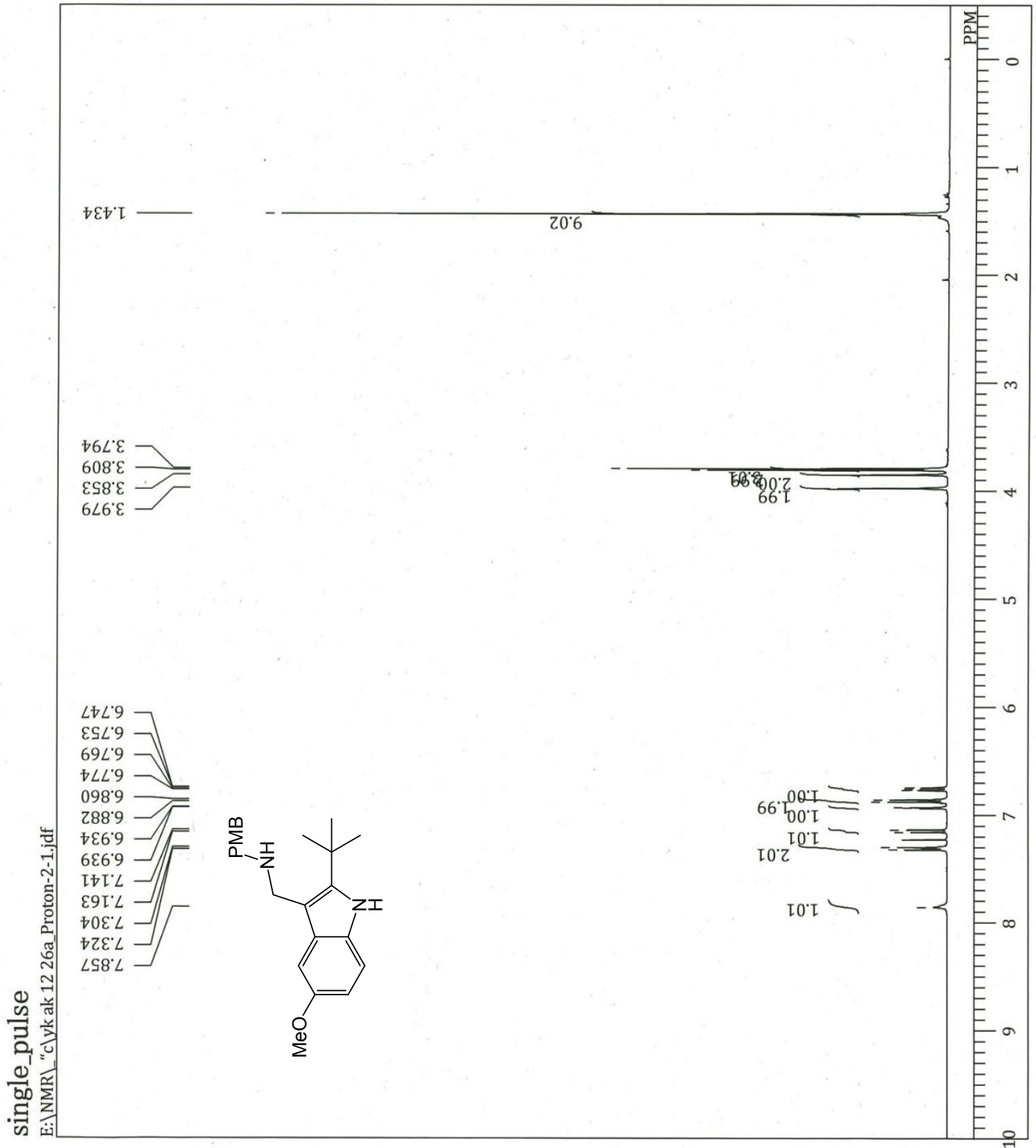


Supporting Information

\section{S1e}

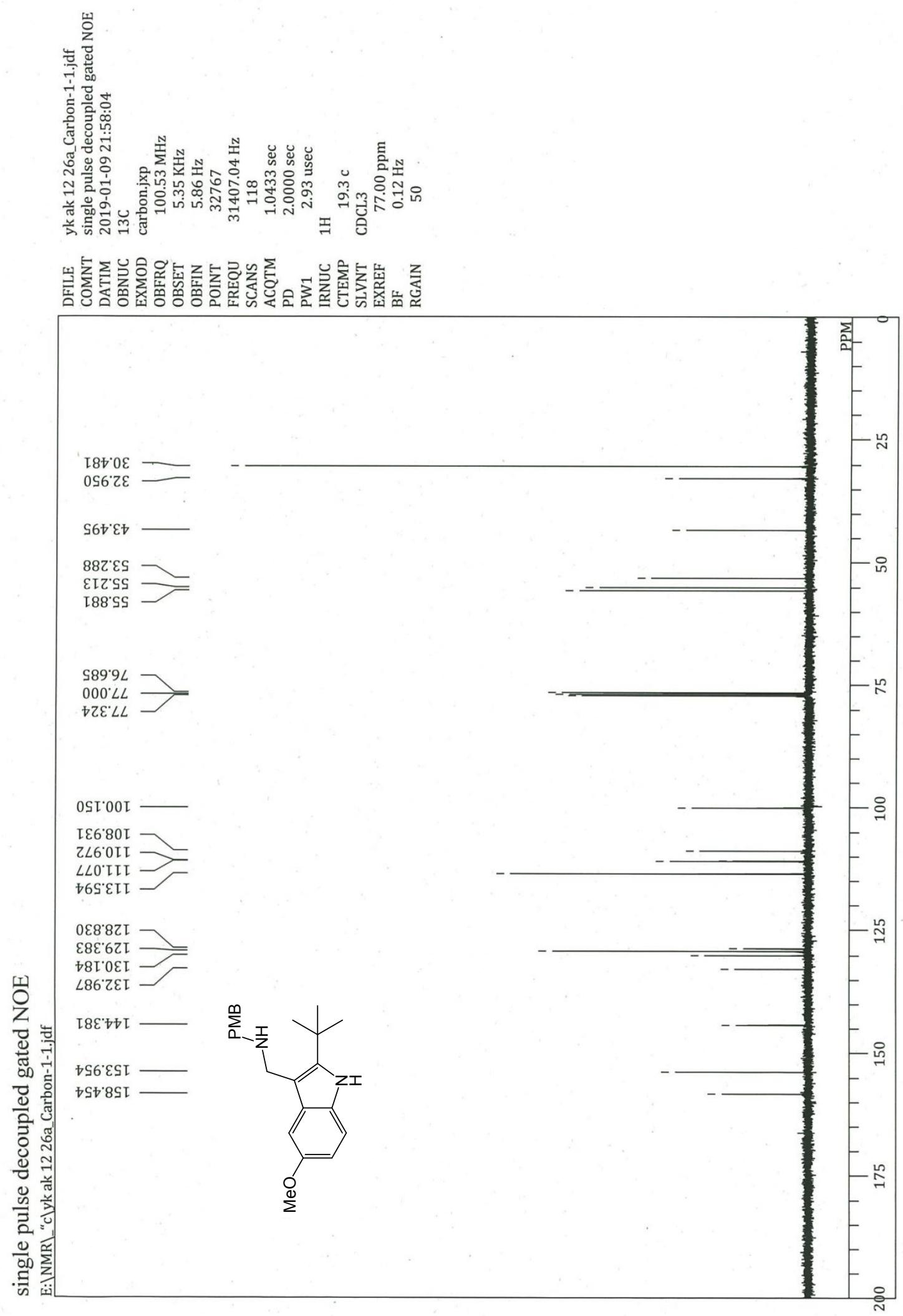


Supporting Information

S1f

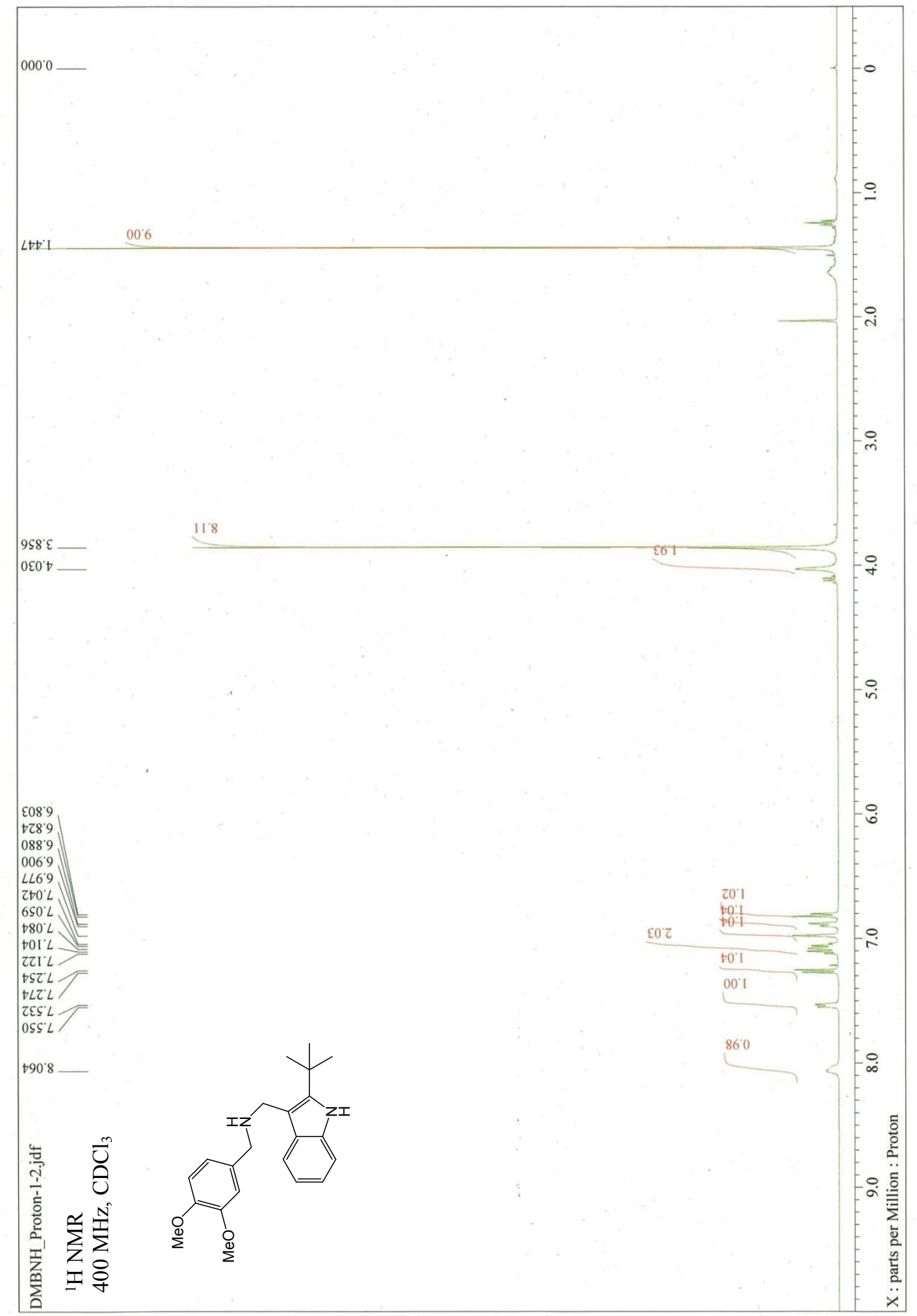


Supporting Information

S1f

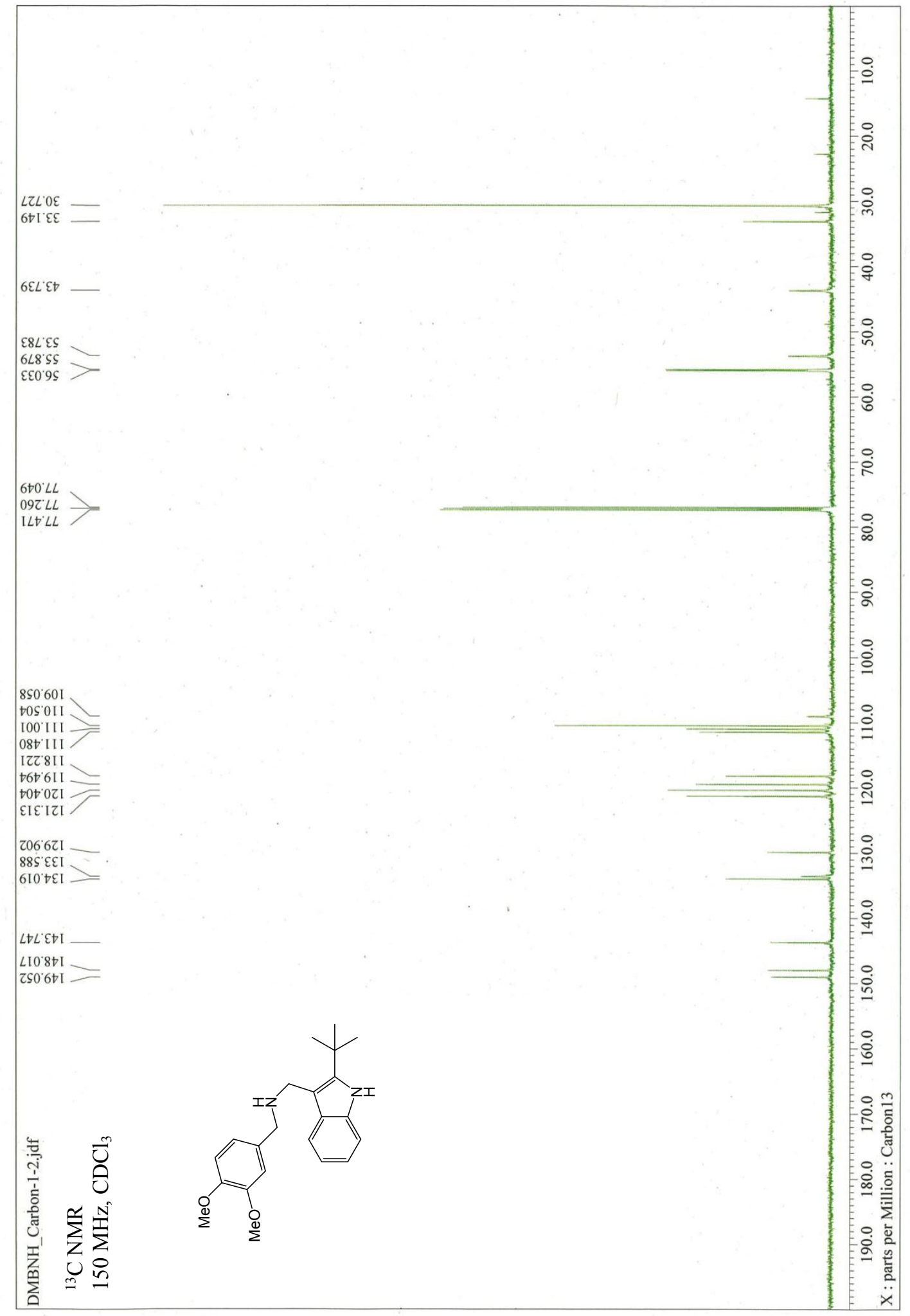


Supporting Information

S1i

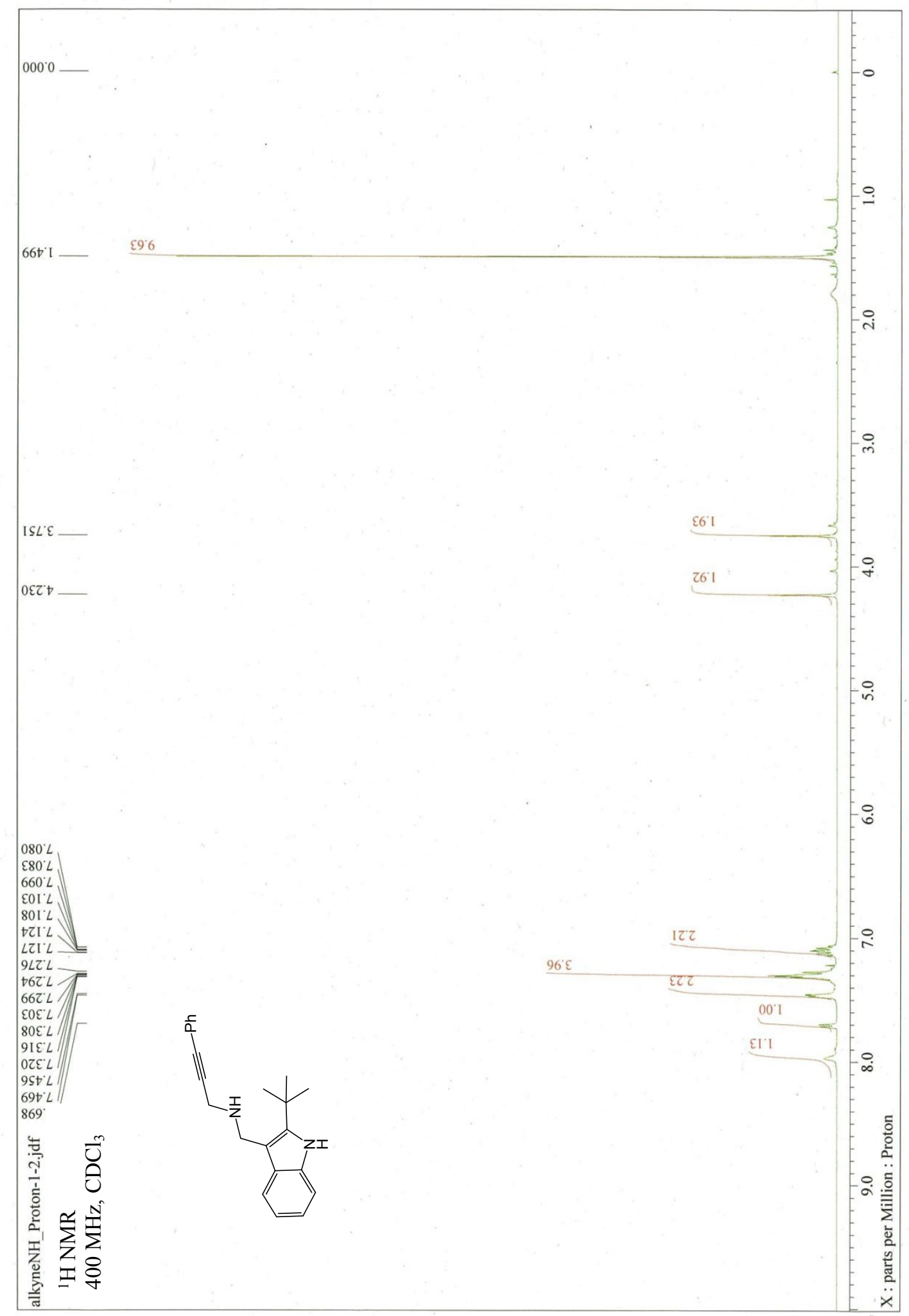


Supporting Information

S1i

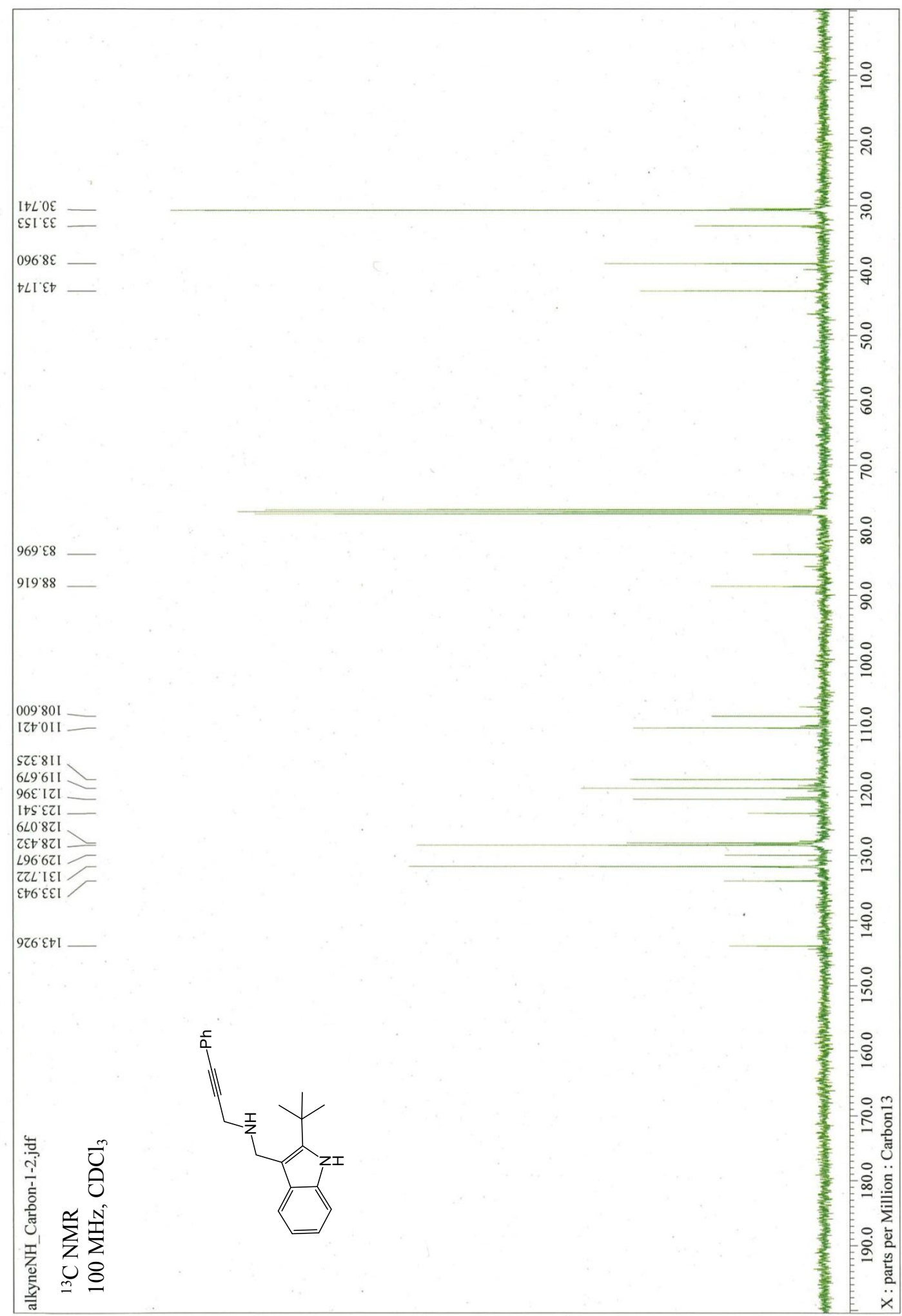


Supporting Information

S1j
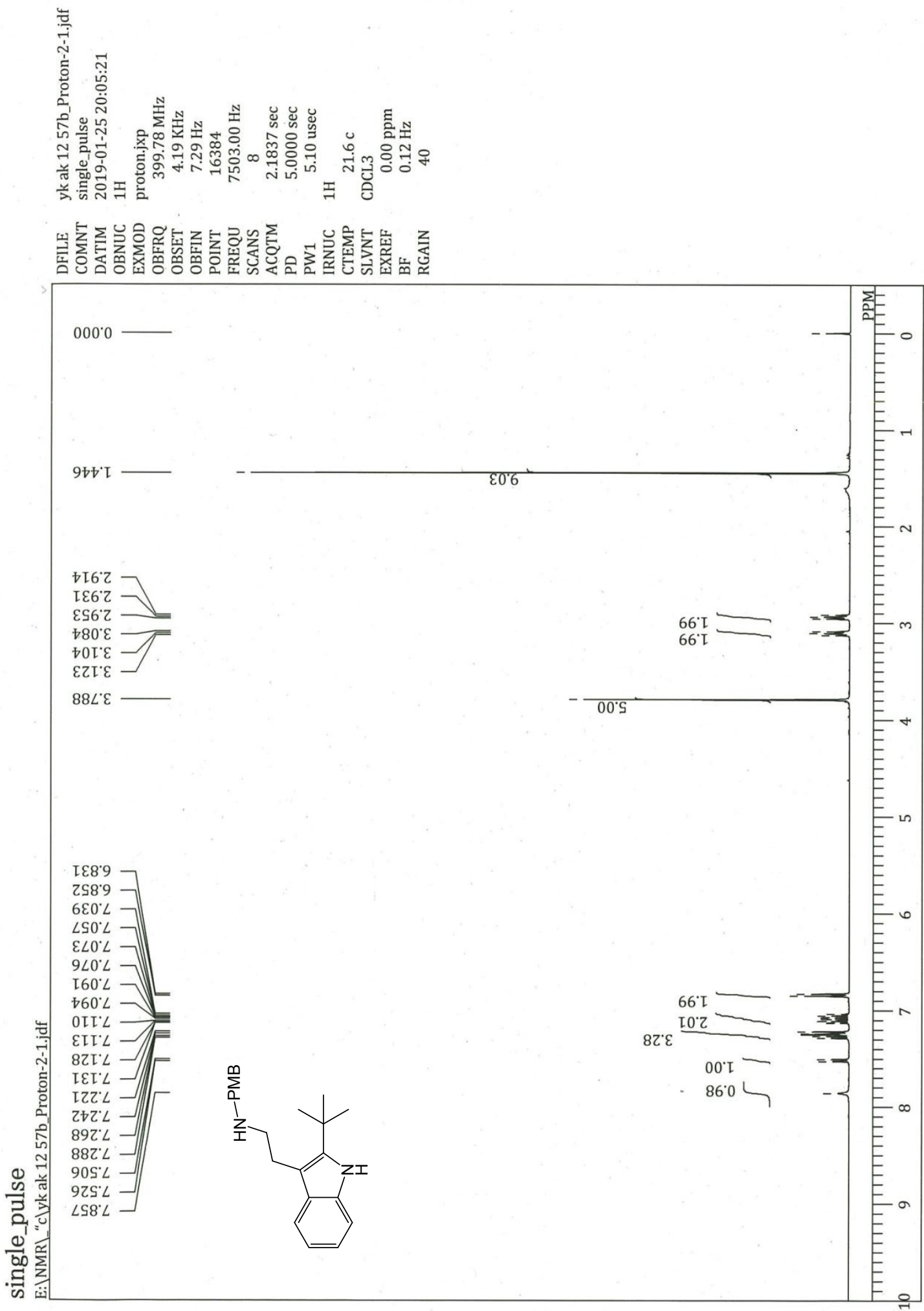
Supporting Information

$\mathrm{S} 1 \mathrm{j}$

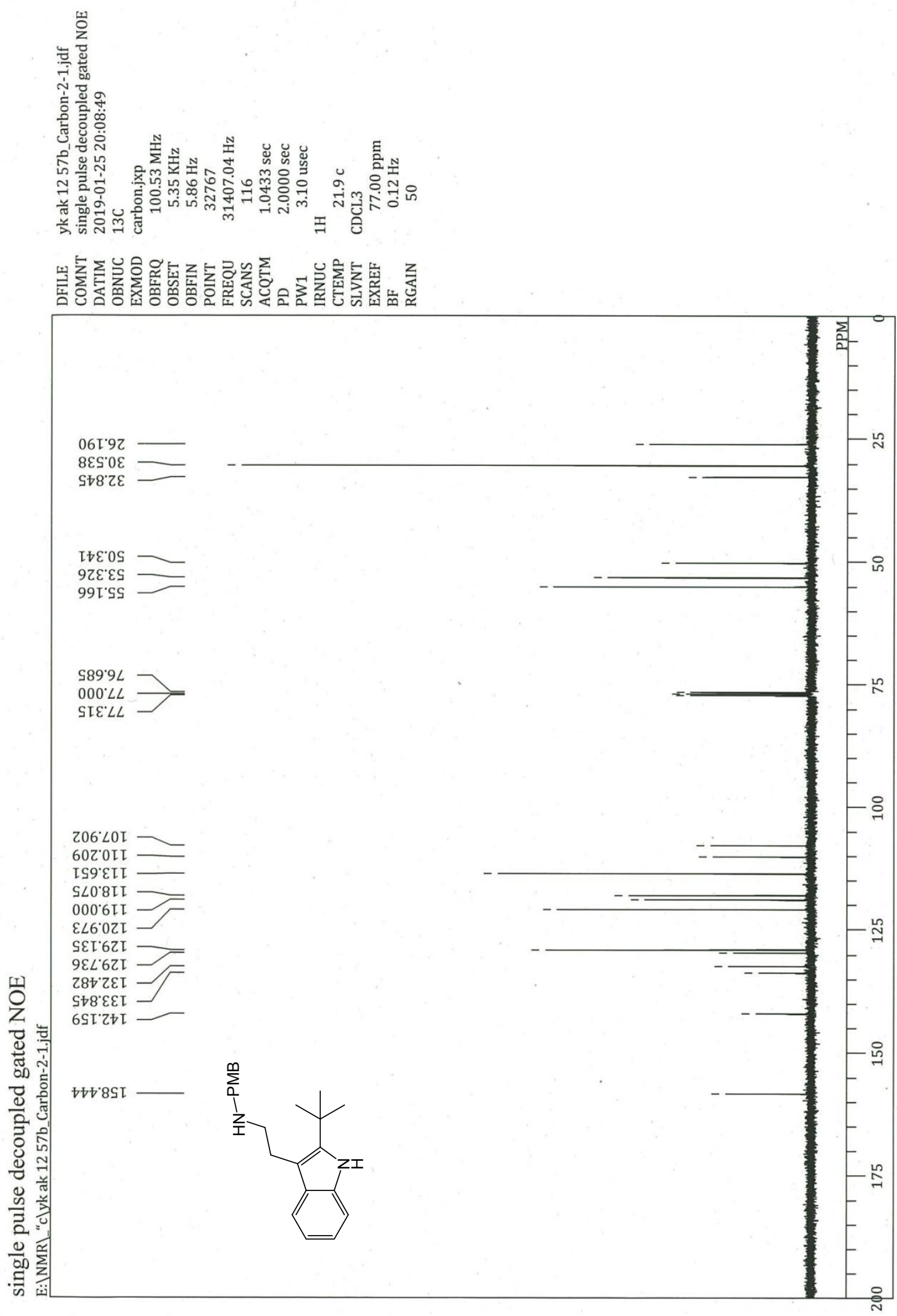


Supporting Information

$1 \mathrm{a}$

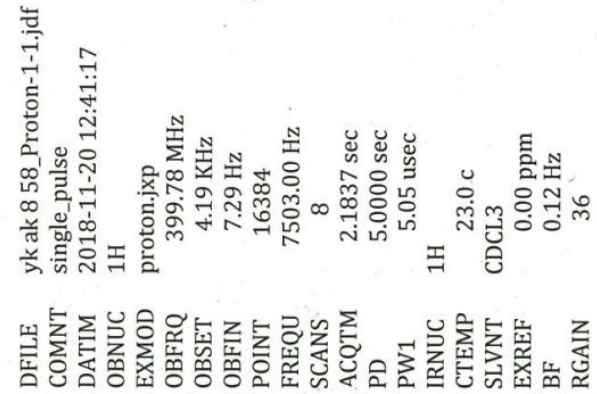

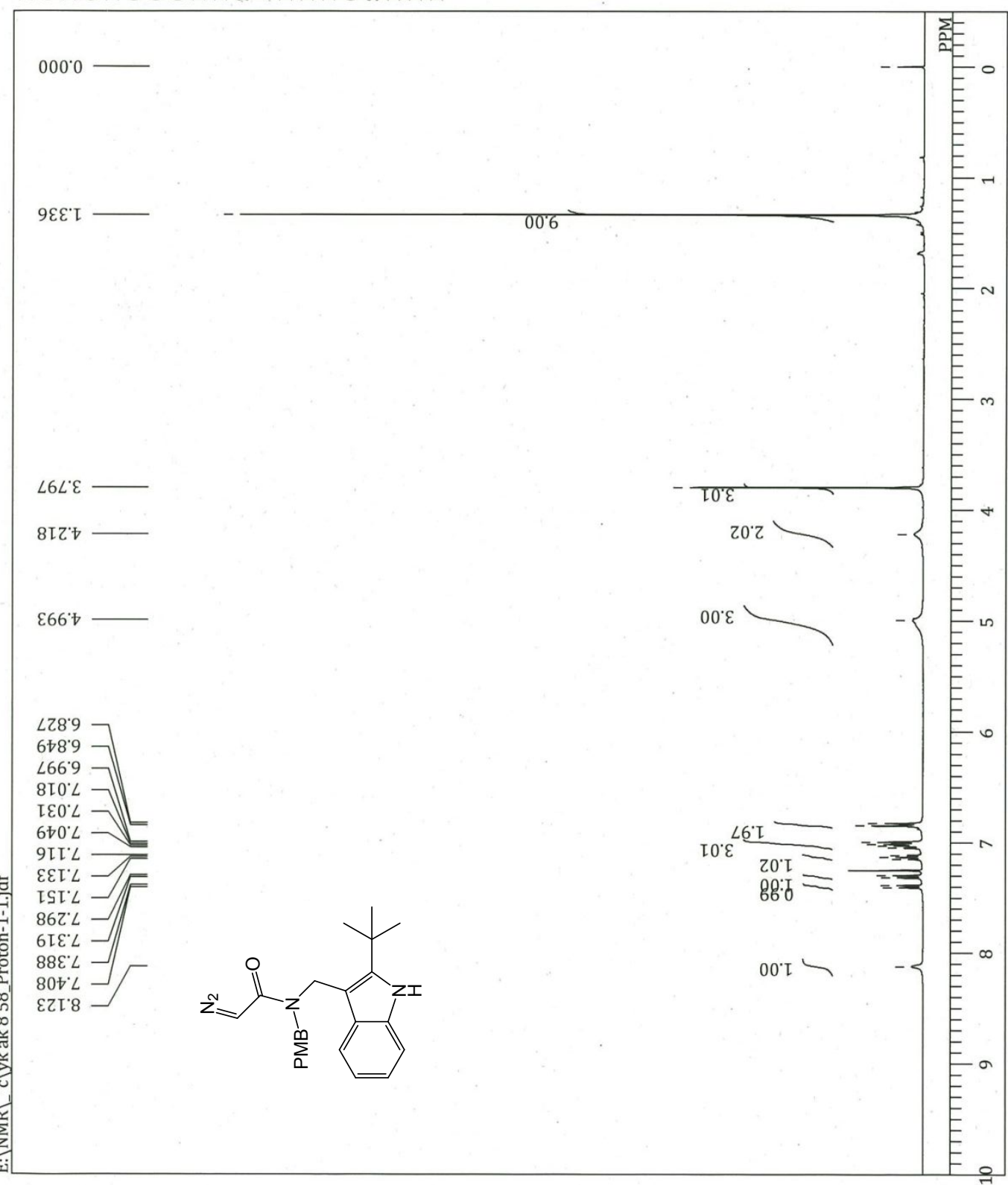


Supporting Information

$1 a$

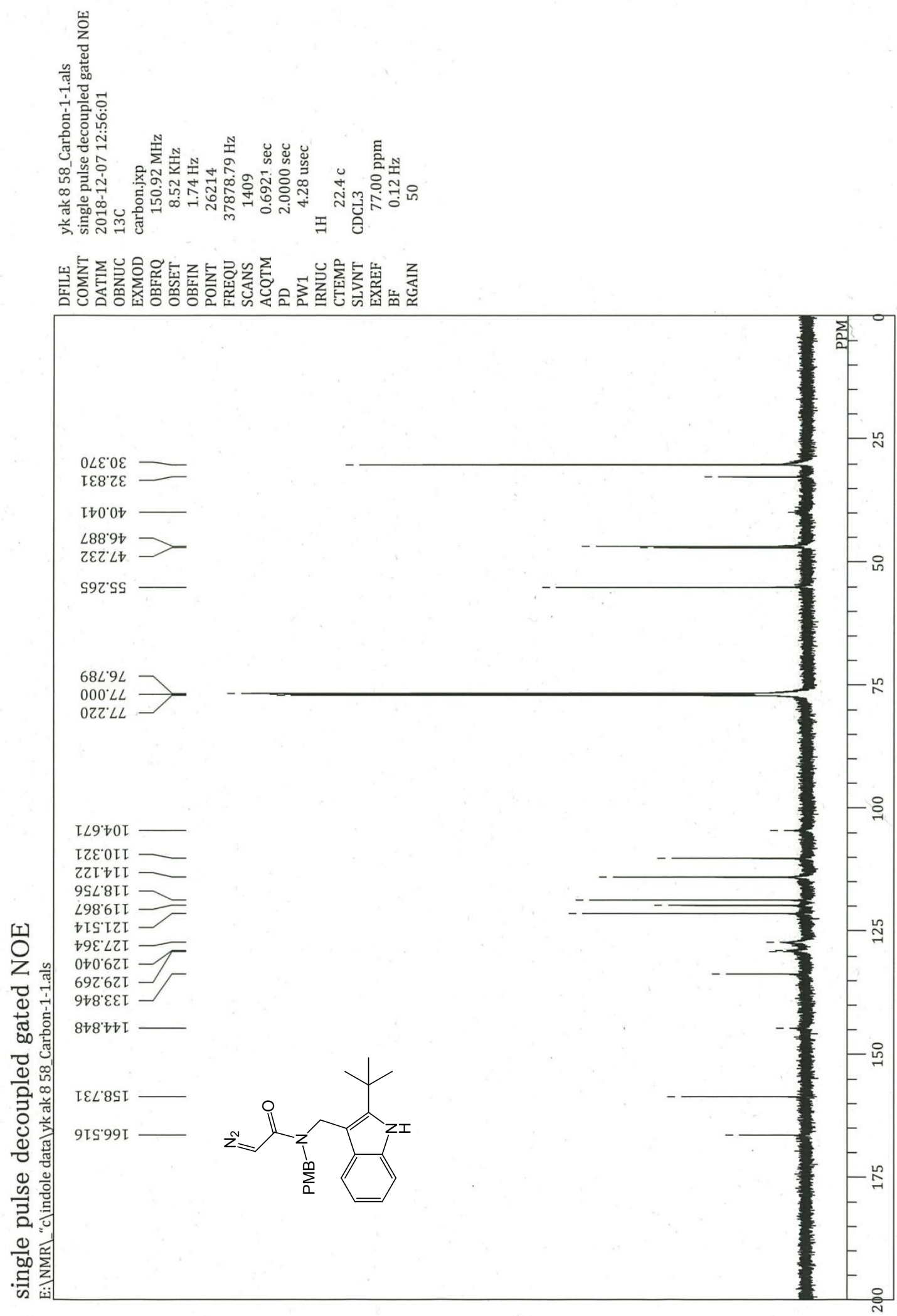


Supporting Information

$1 b$ 
Supporting Information

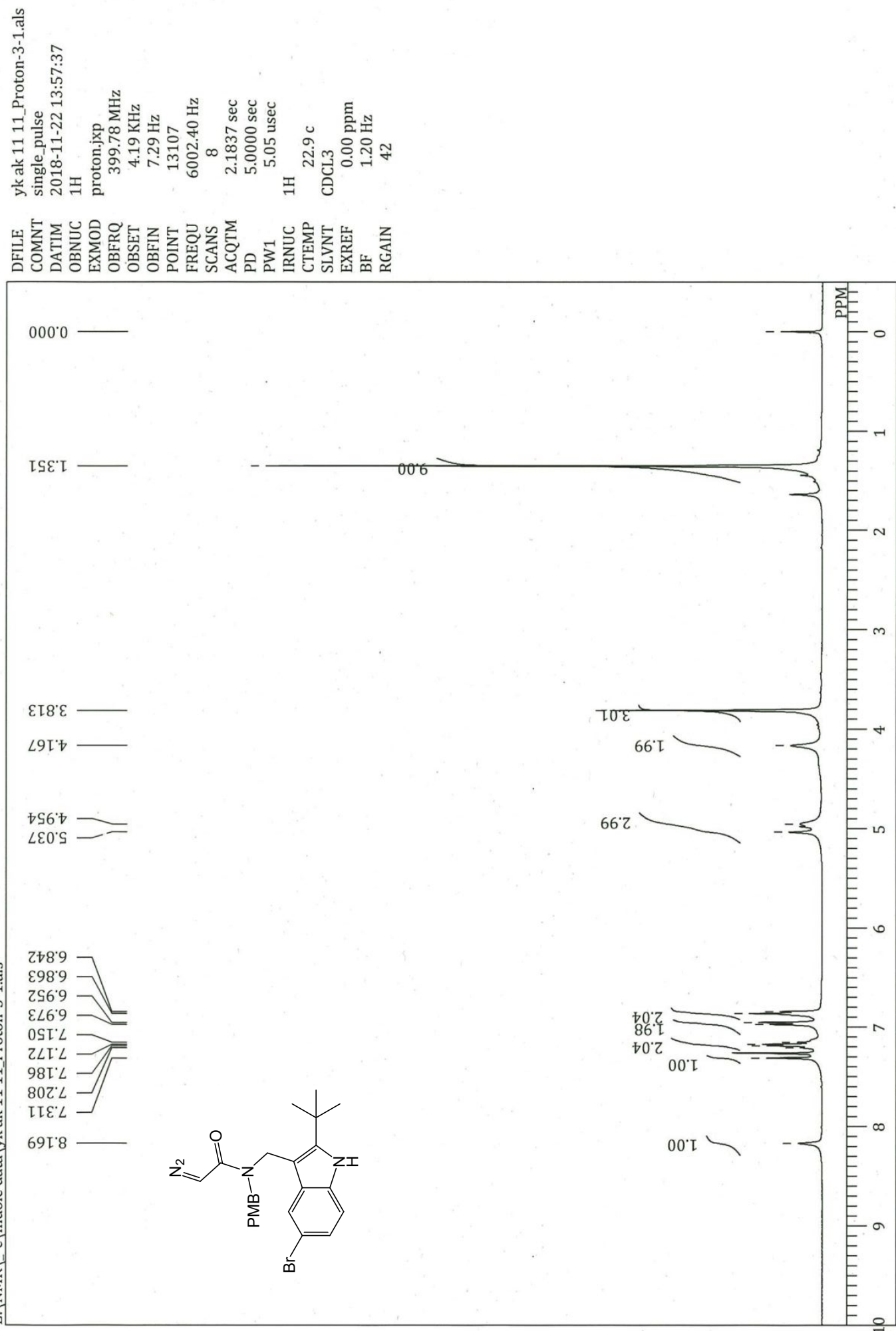


Supporting Information

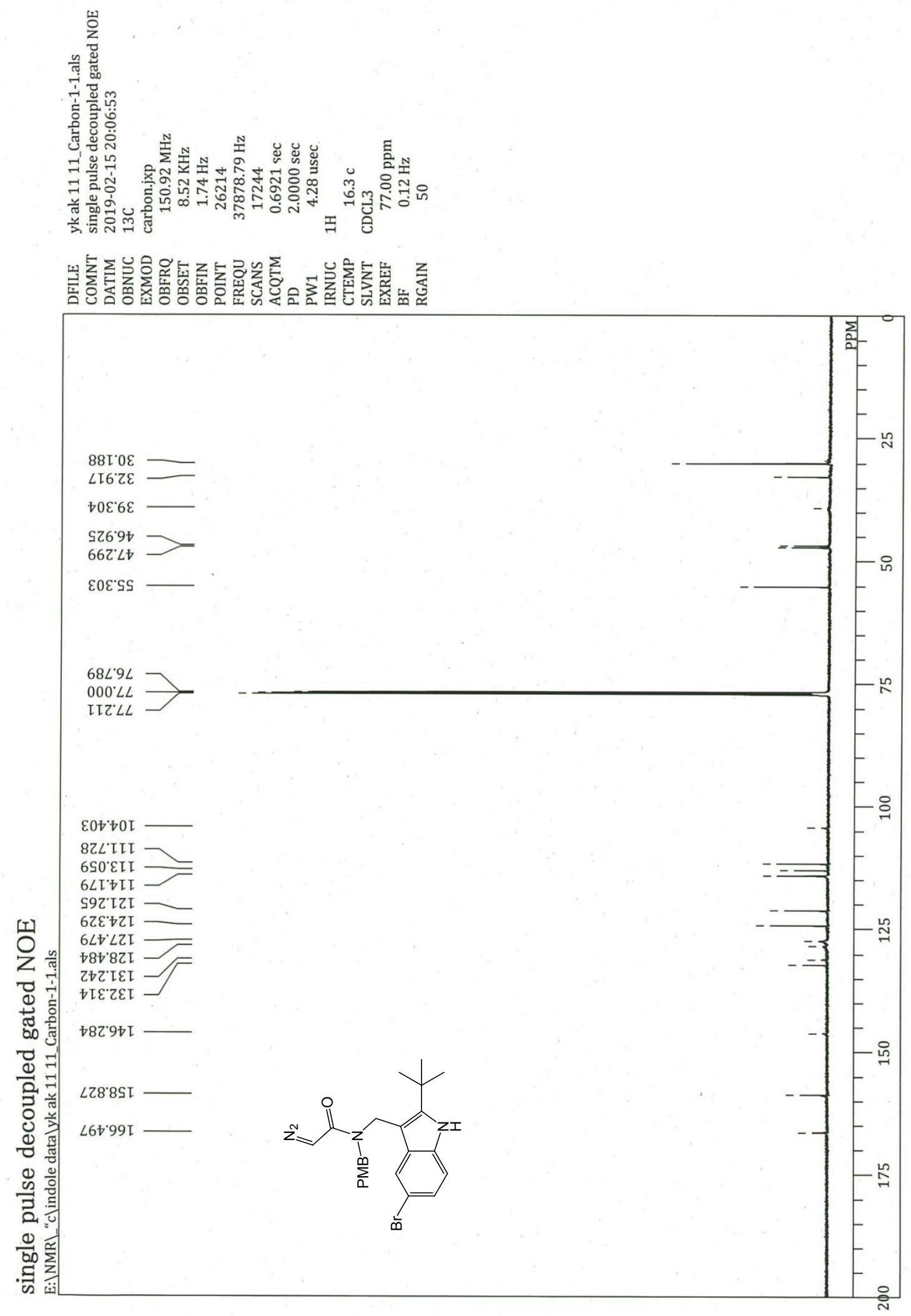


Supporting Information

$1 c$

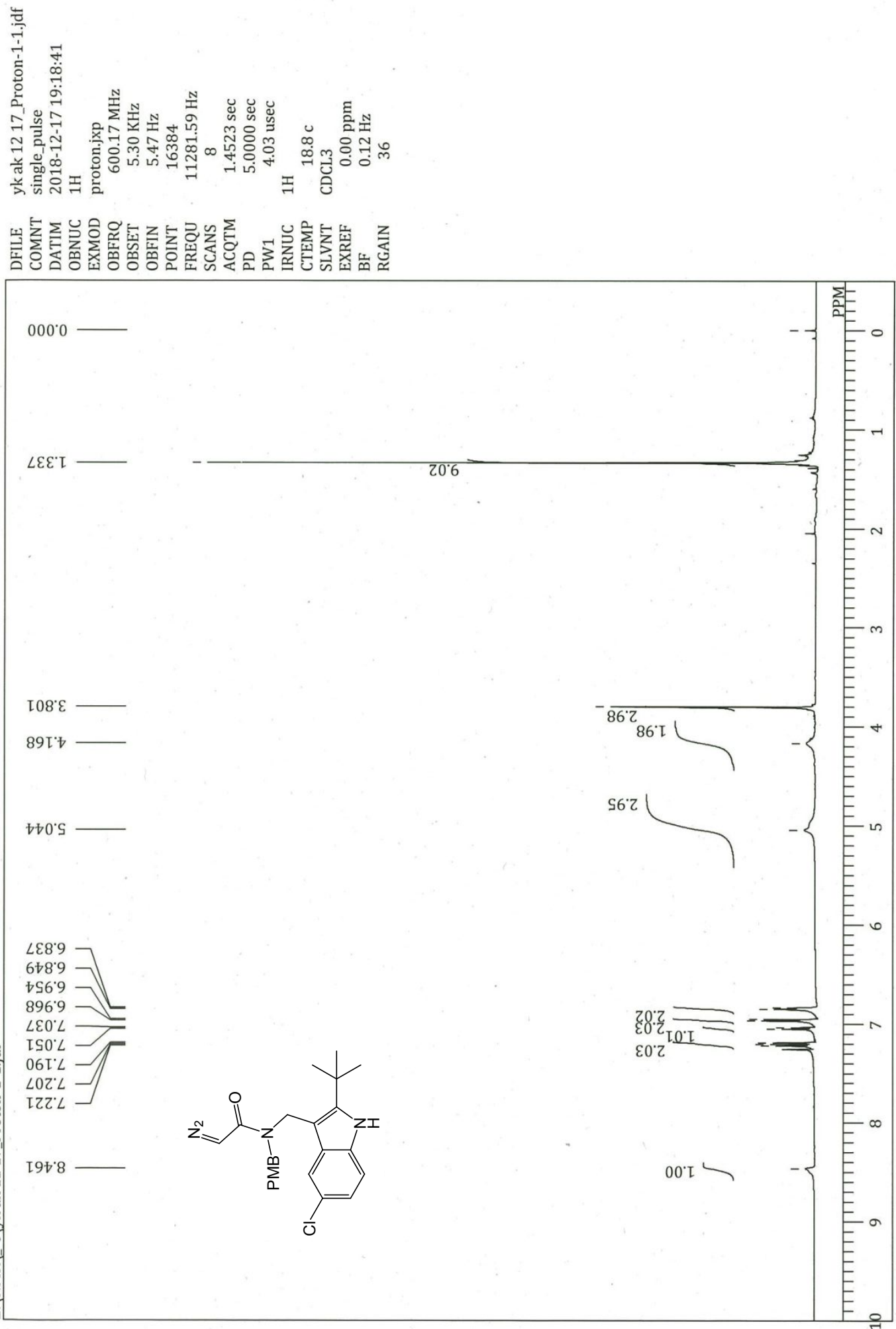


Supporting Information

$1 c$

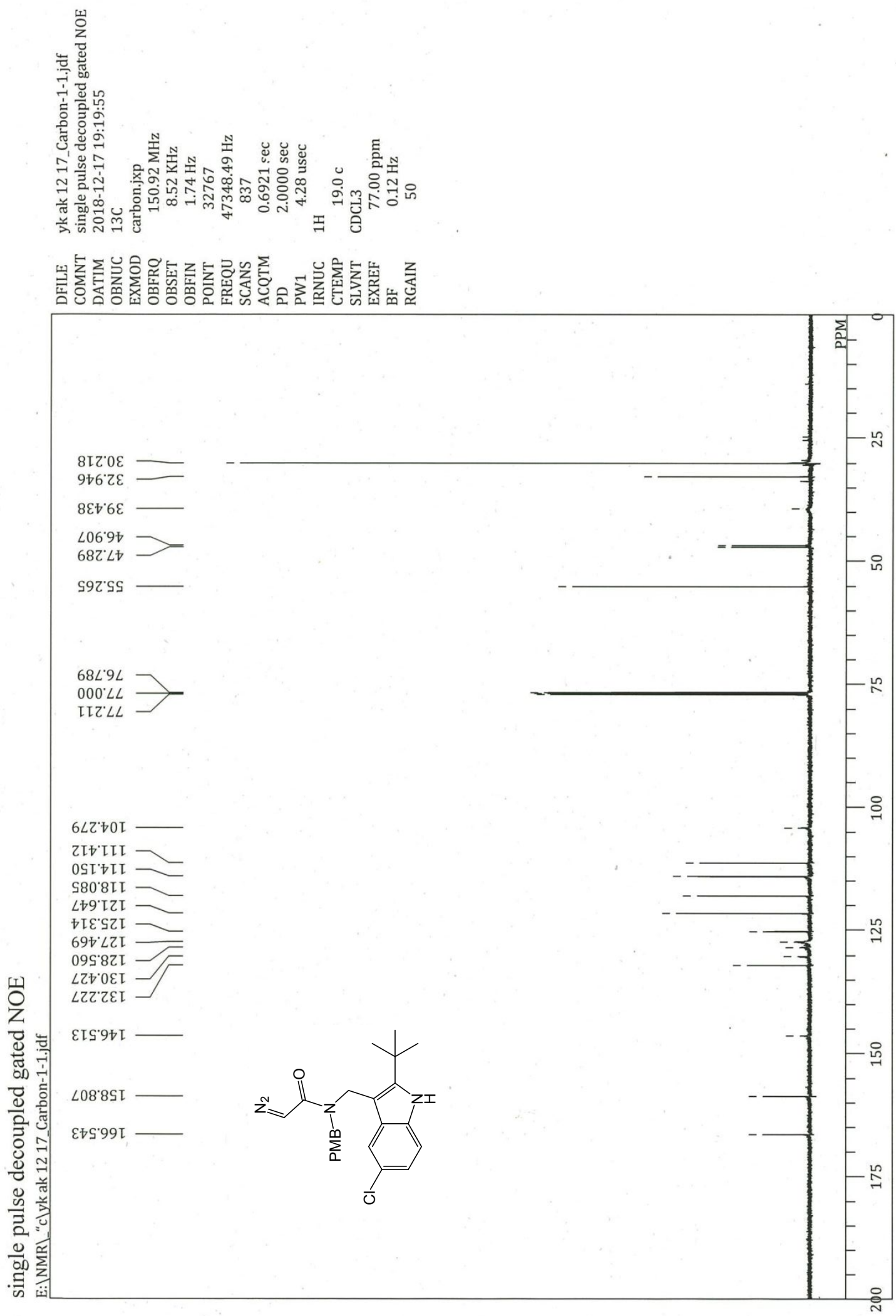


Supporting Information

$1 d$

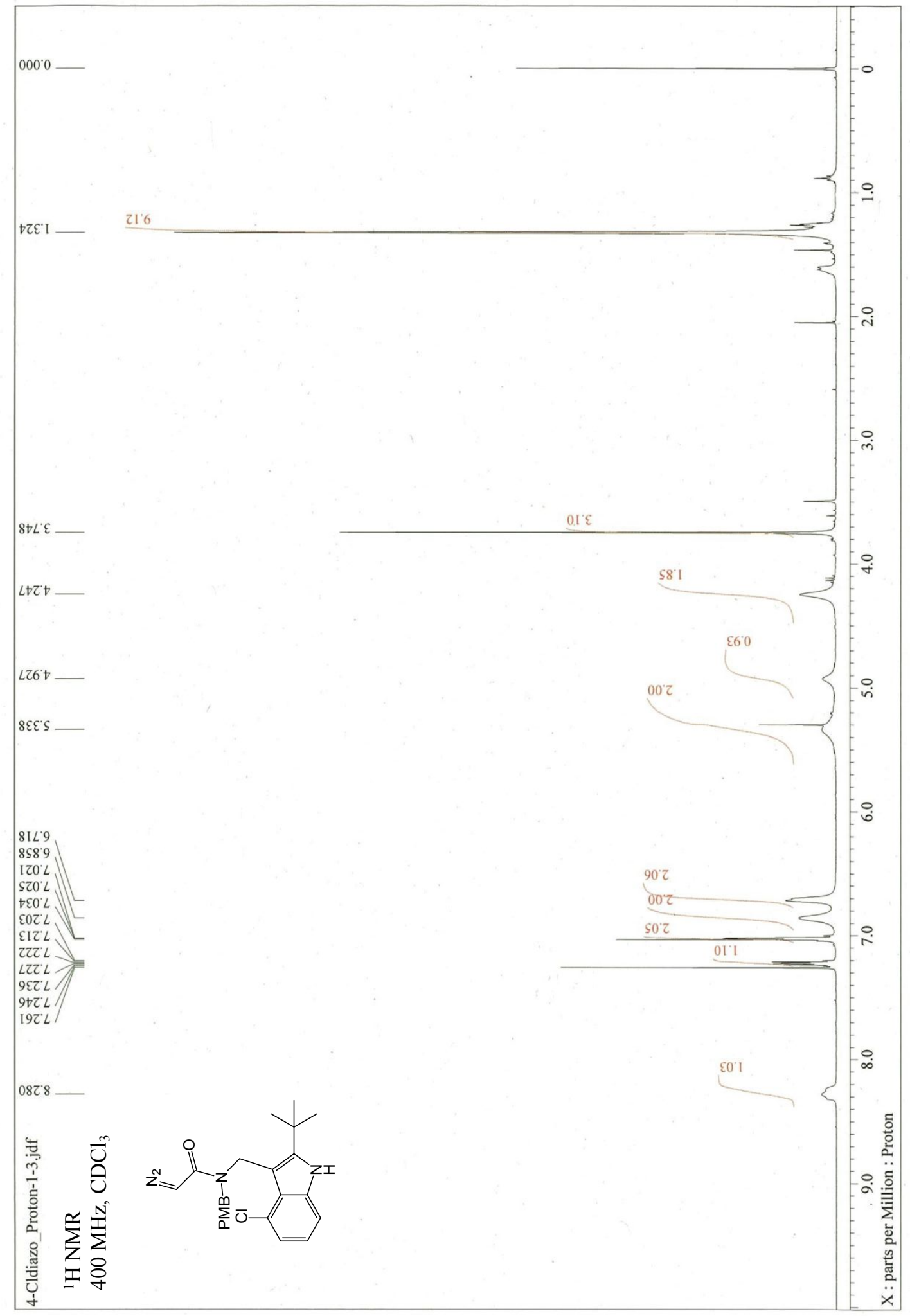


Supporting Information

$1 d$

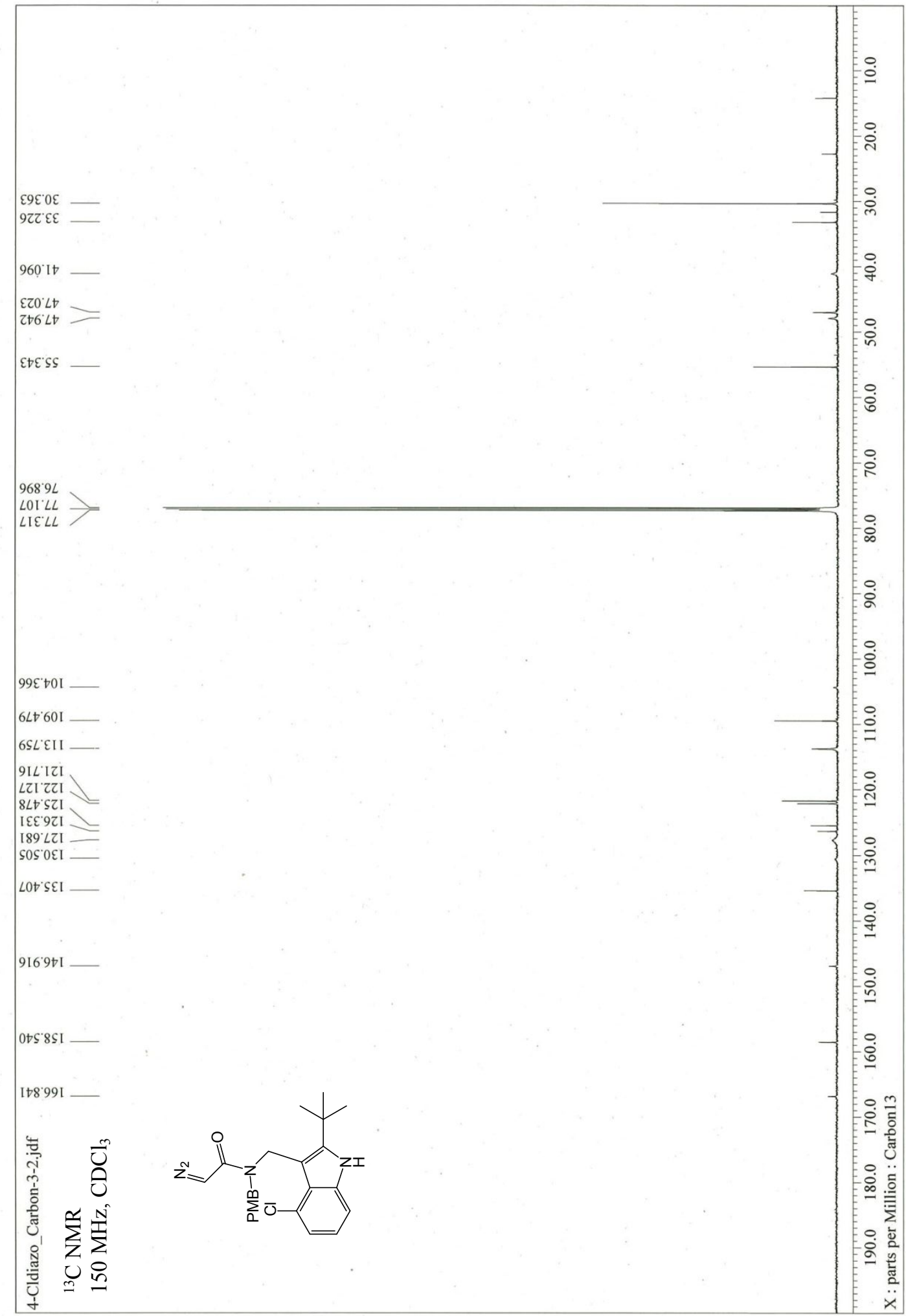


Supporting Information

$1 e$

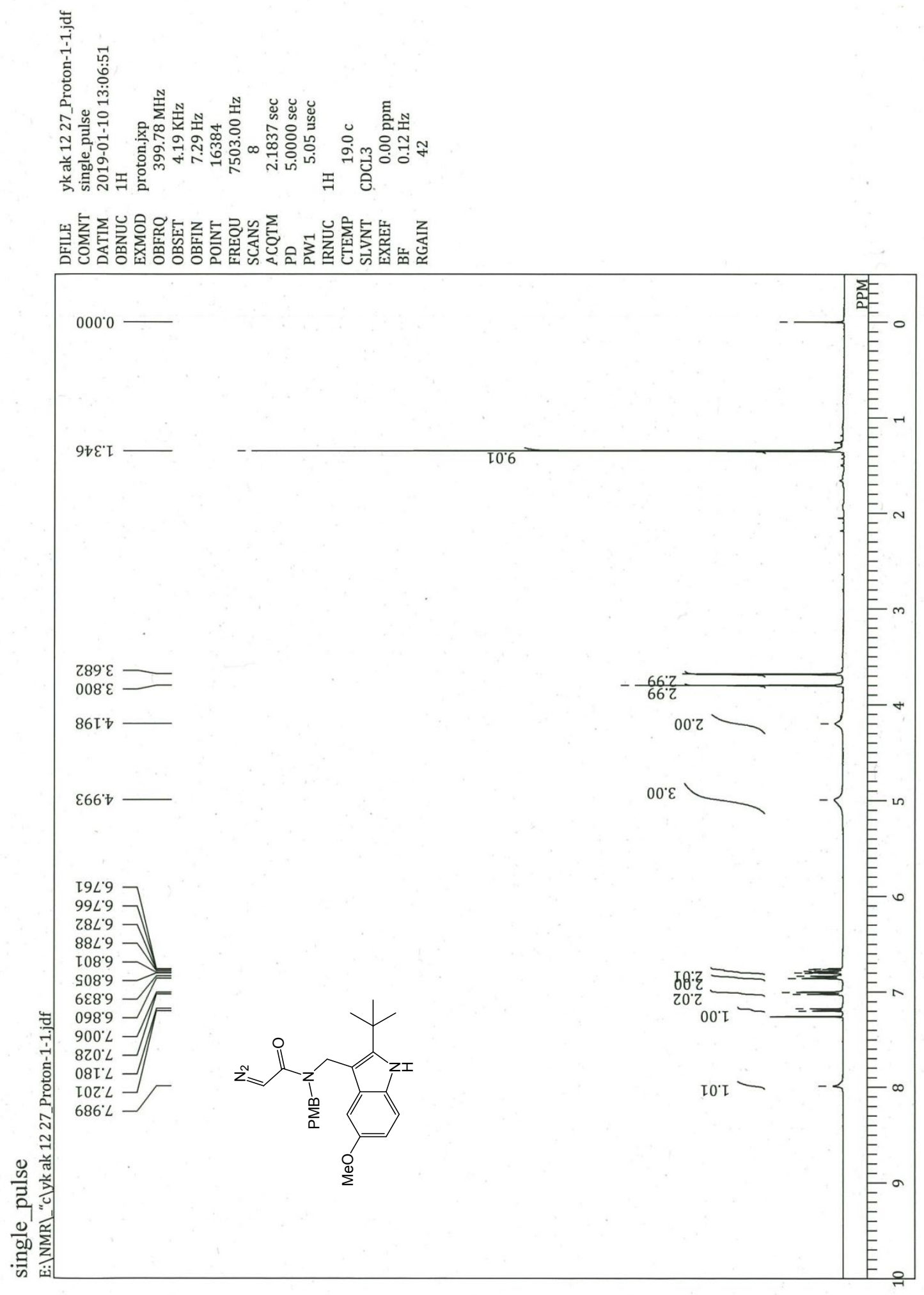


Supporting Information

$1 e$

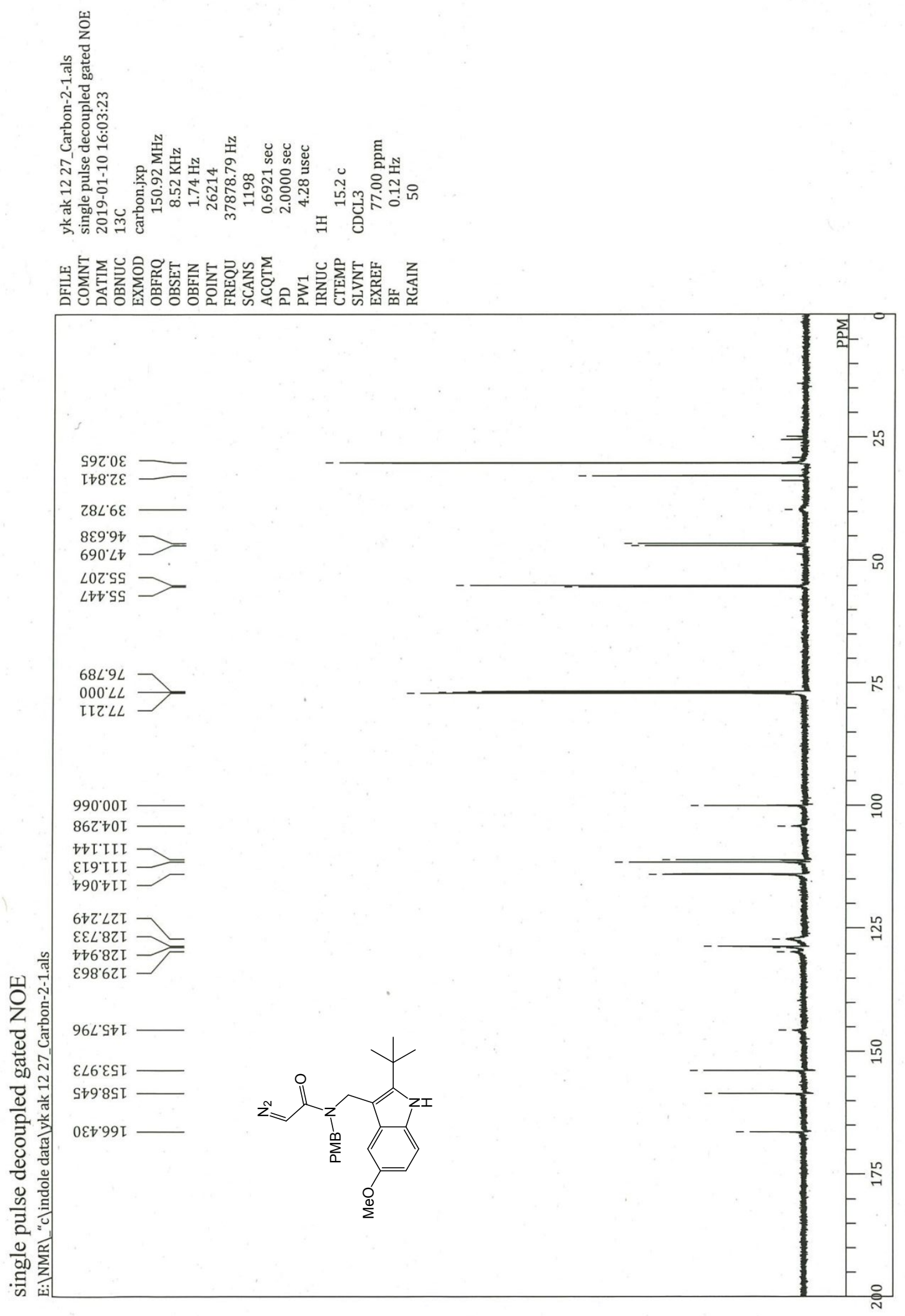


Supporting Information

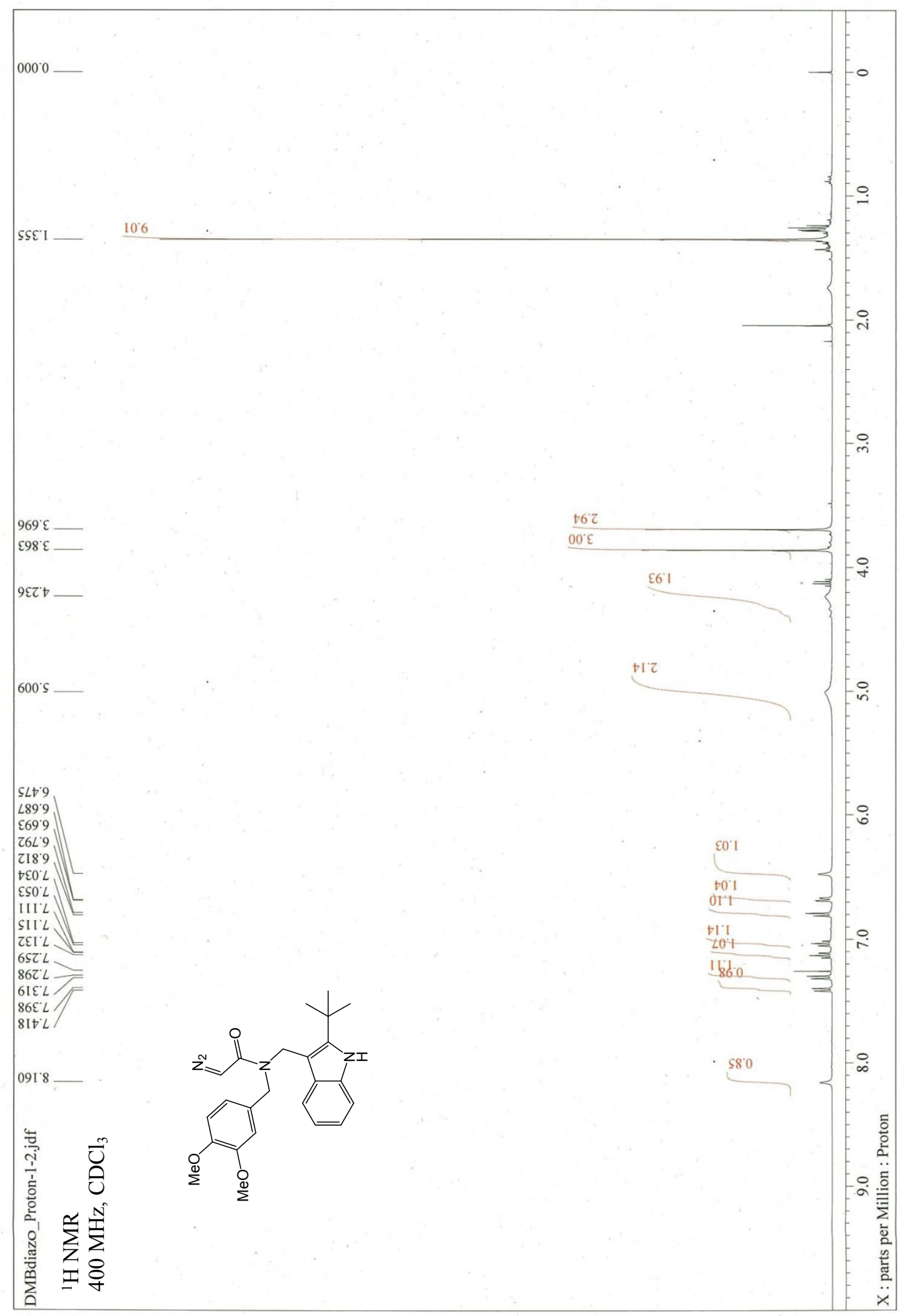


Supporting Information

$1 f$

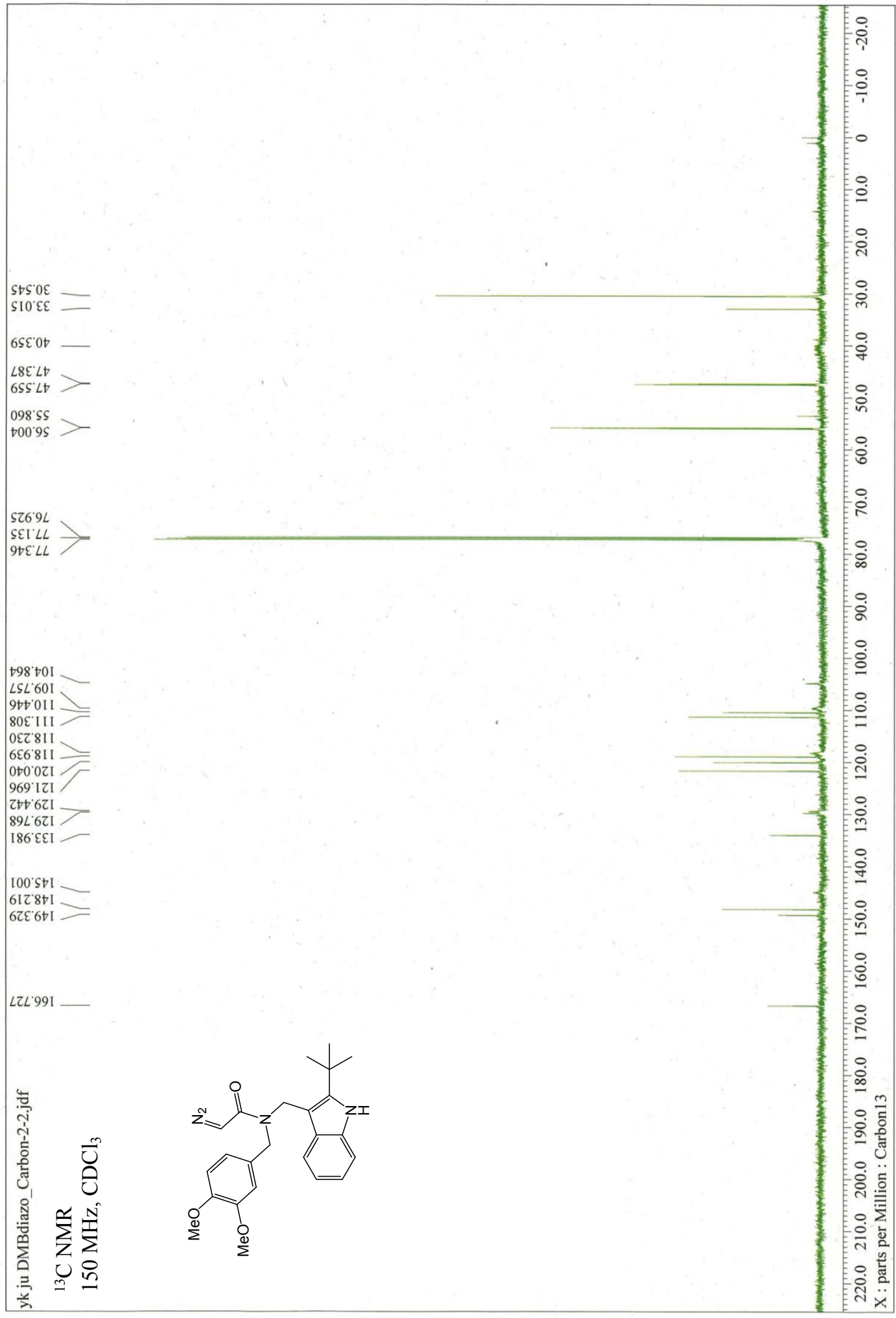


Supporting Information

$1 \mathrm{~g}$

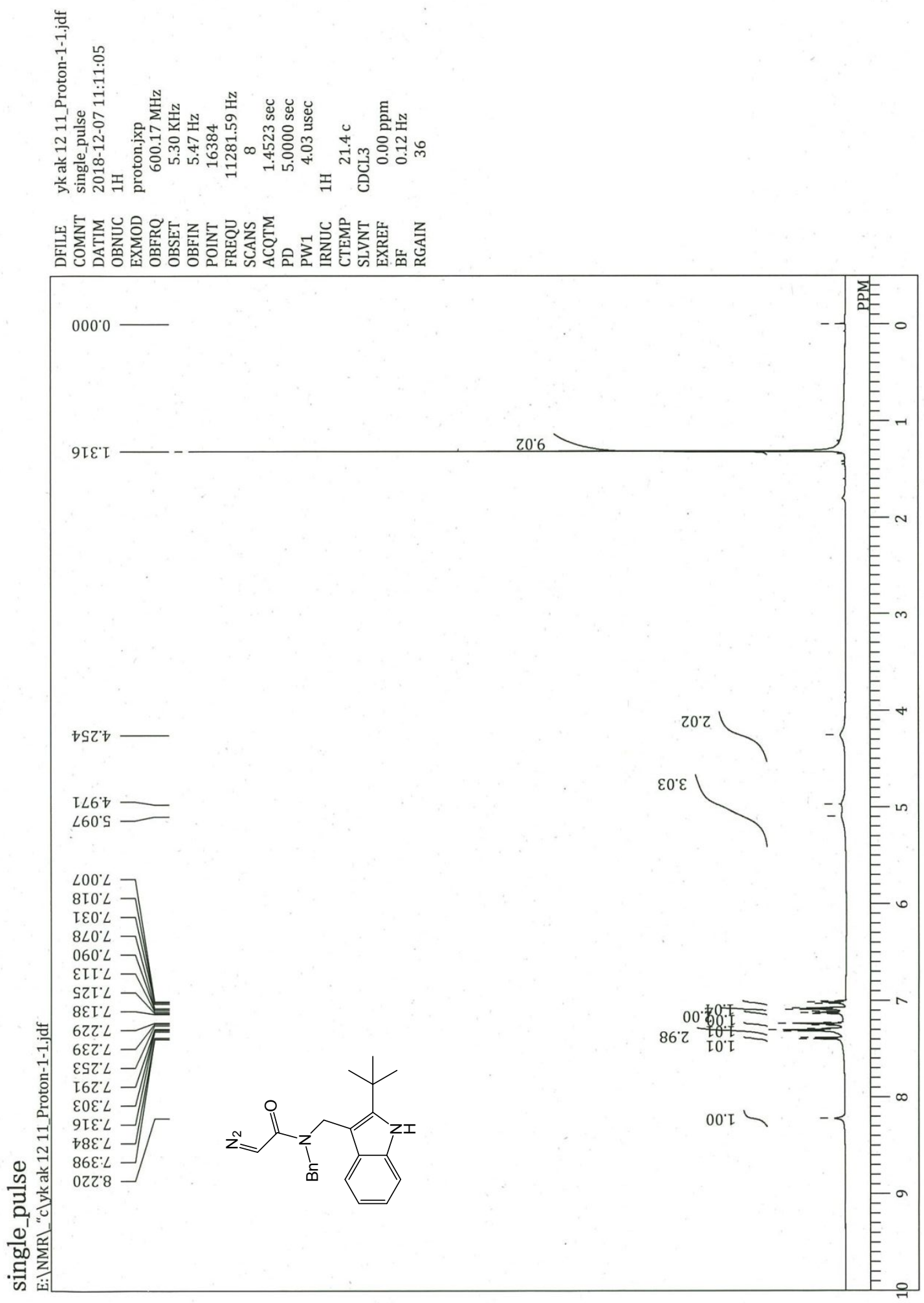


Supporting Information

$1 \mathrm{~g}$

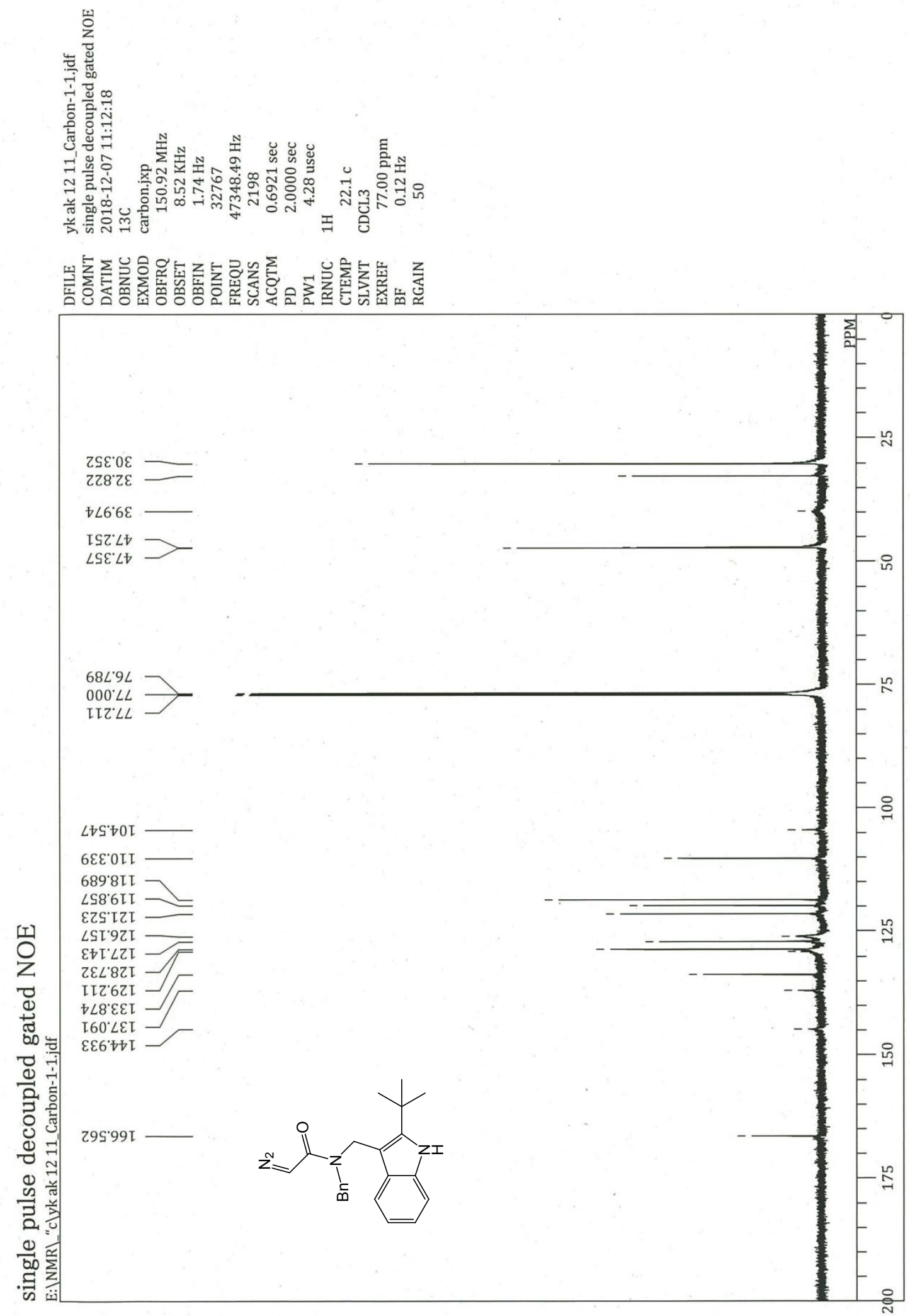


Supporting Information

$1 \mathrm{~h}$

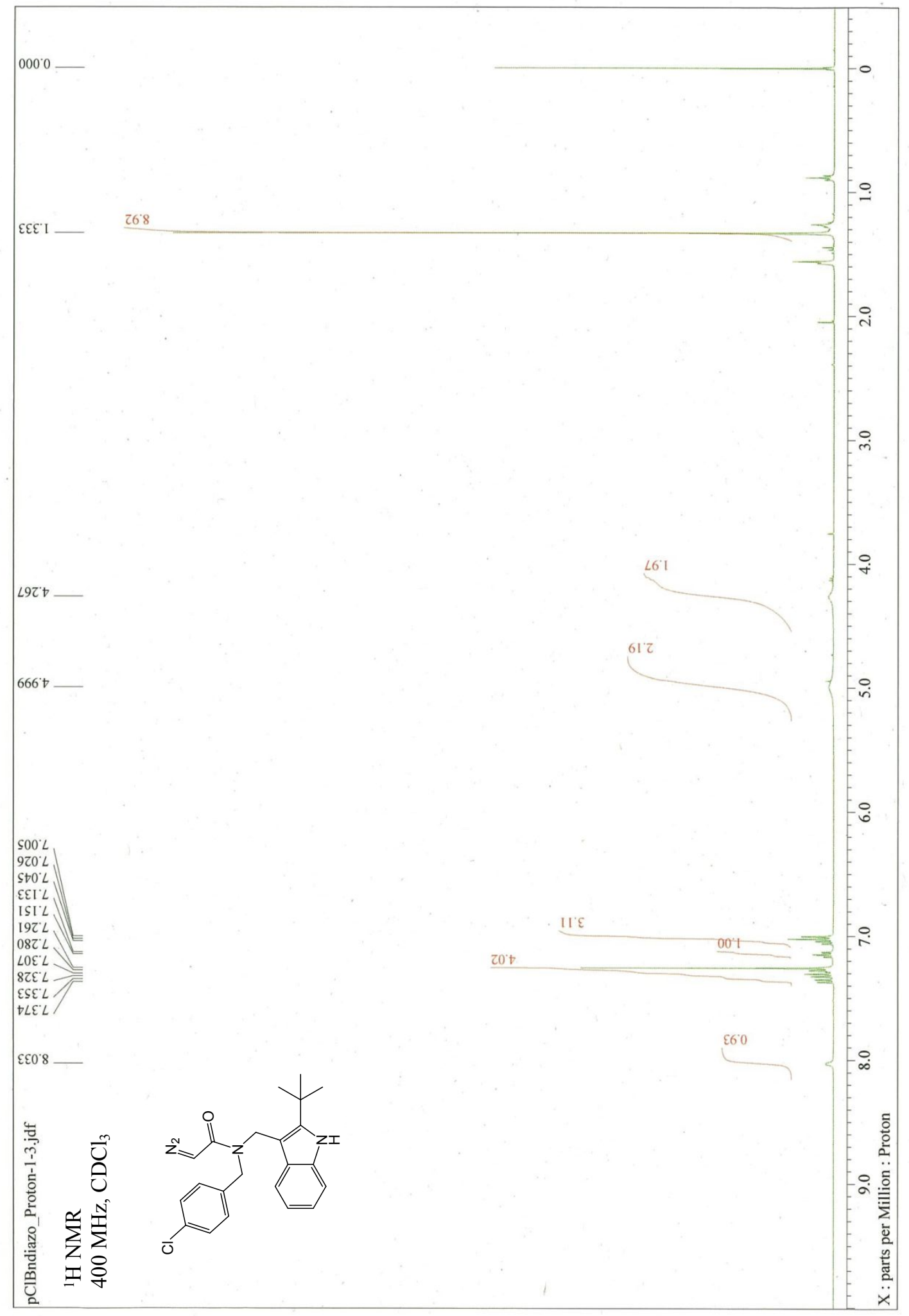


Supporting Information

$1 \mathrm{~h}$

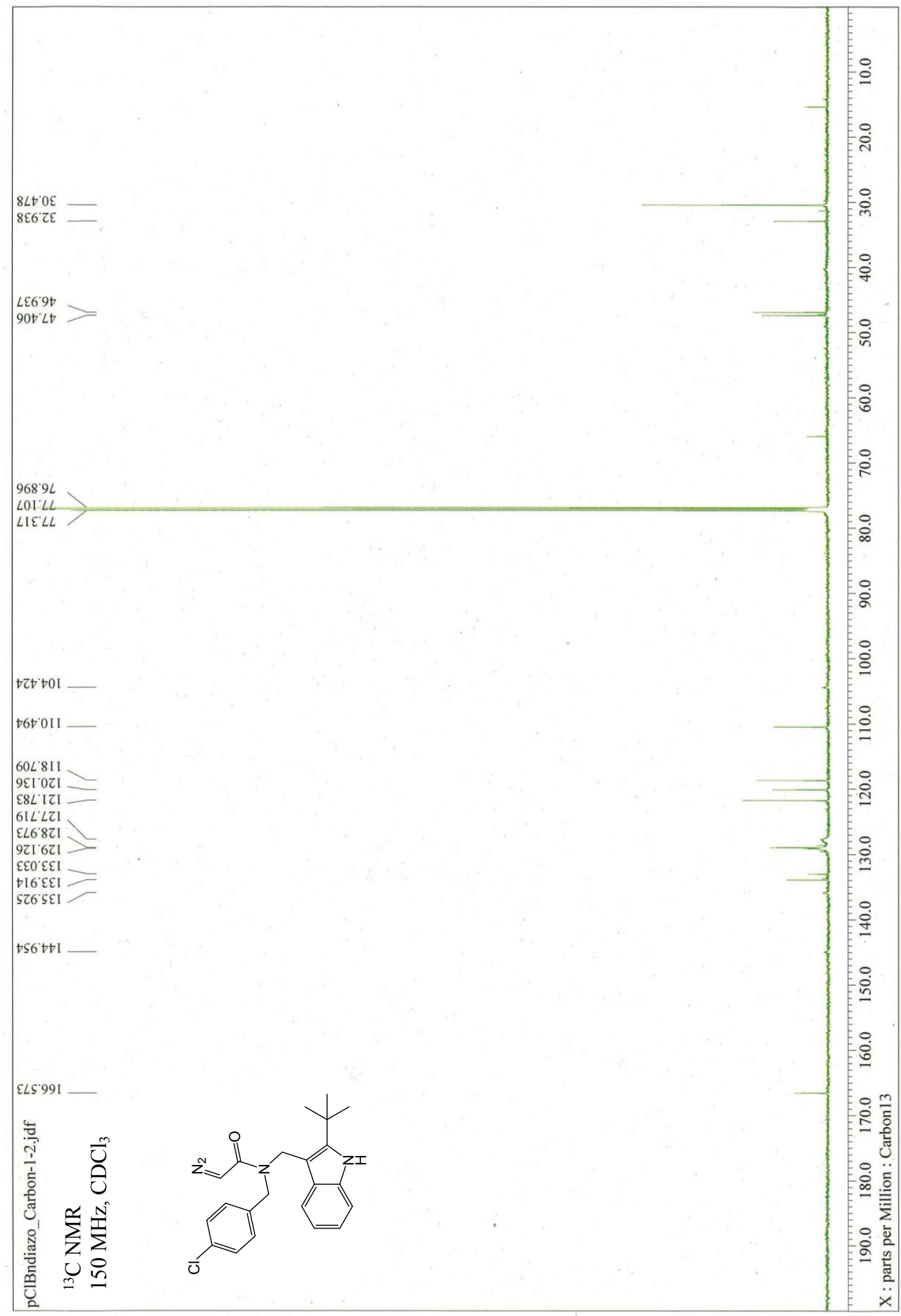


Supporting Information

$1 \mathrm{i}$

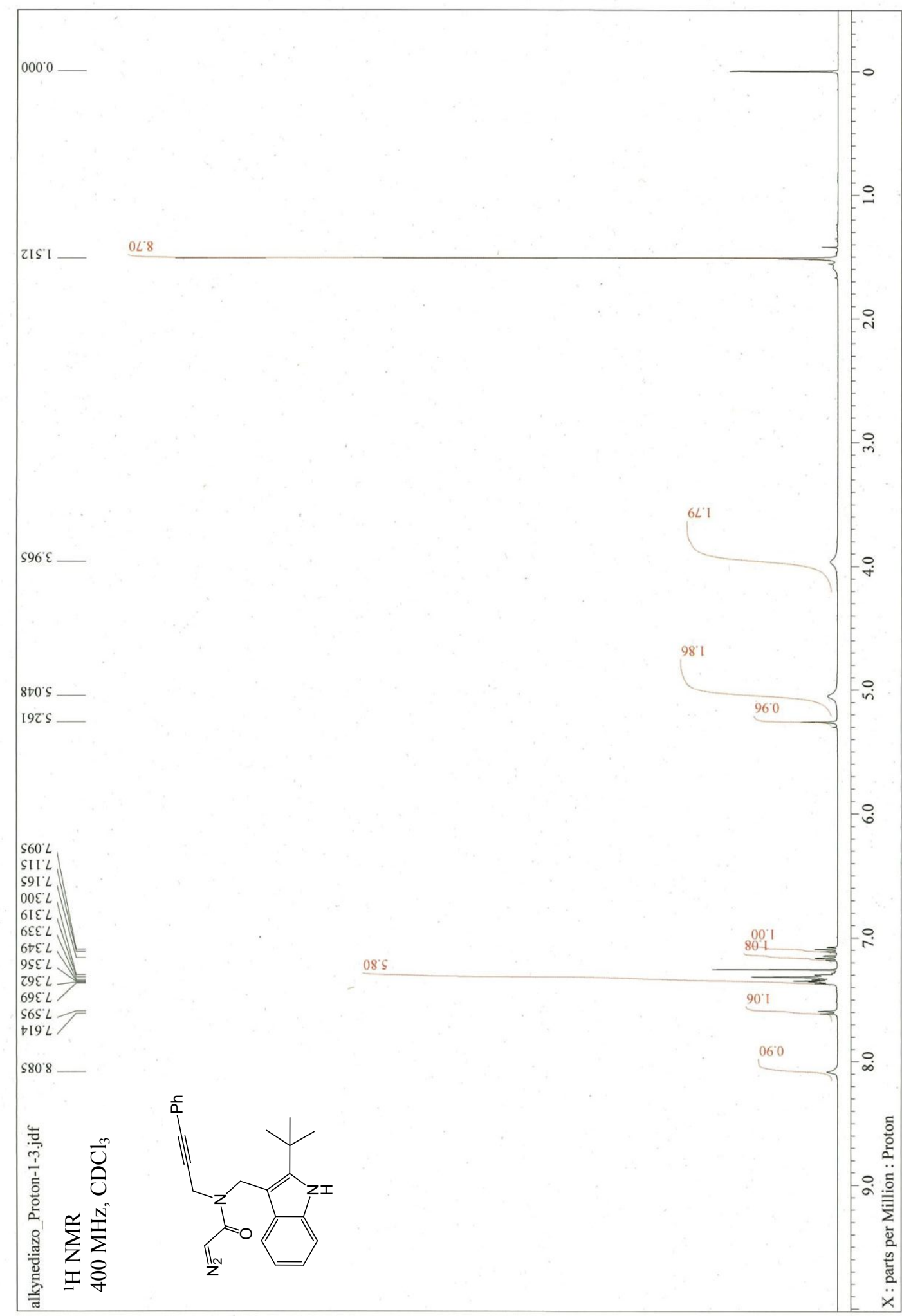


Supporting Information

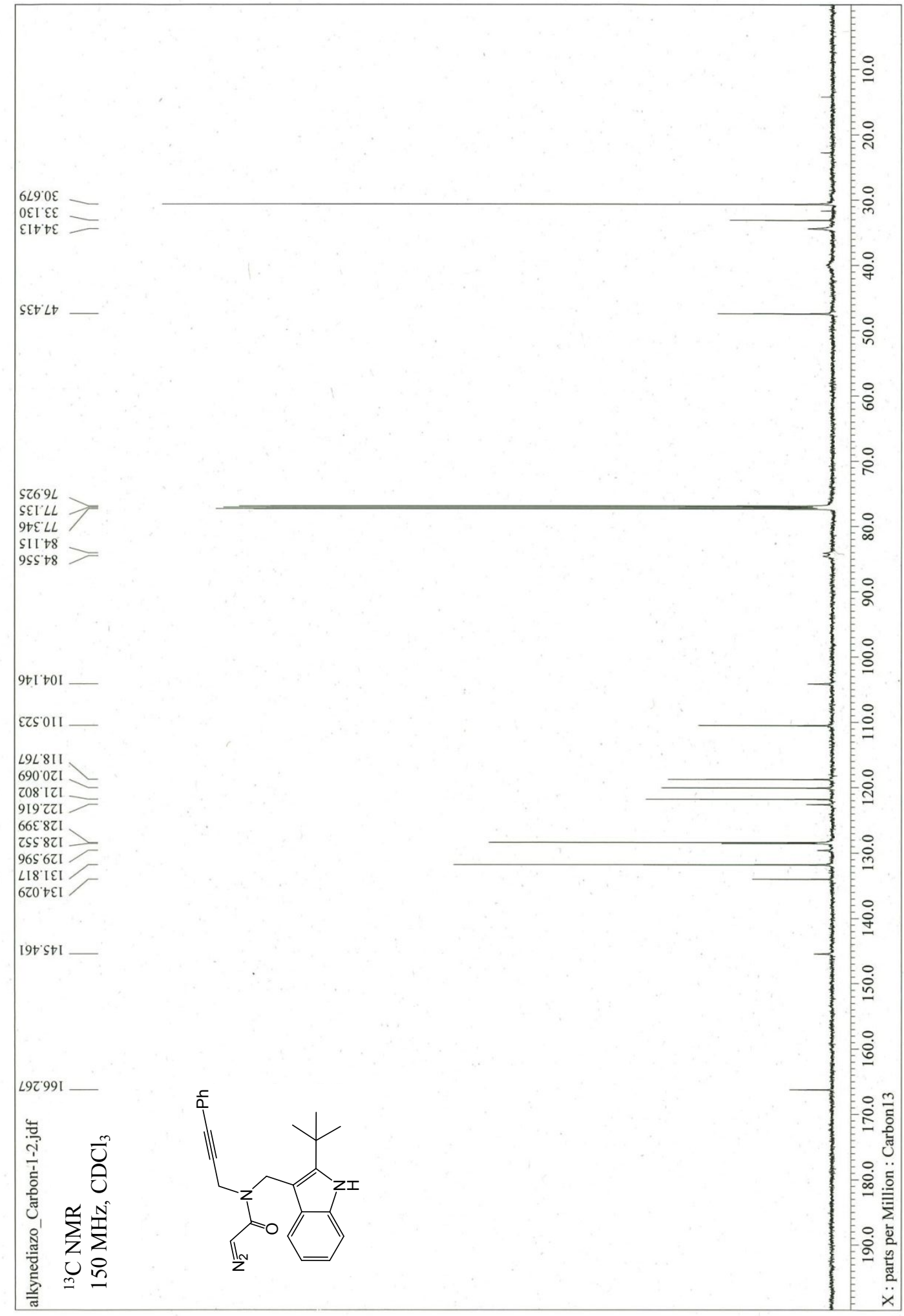


Supporting Information

1j
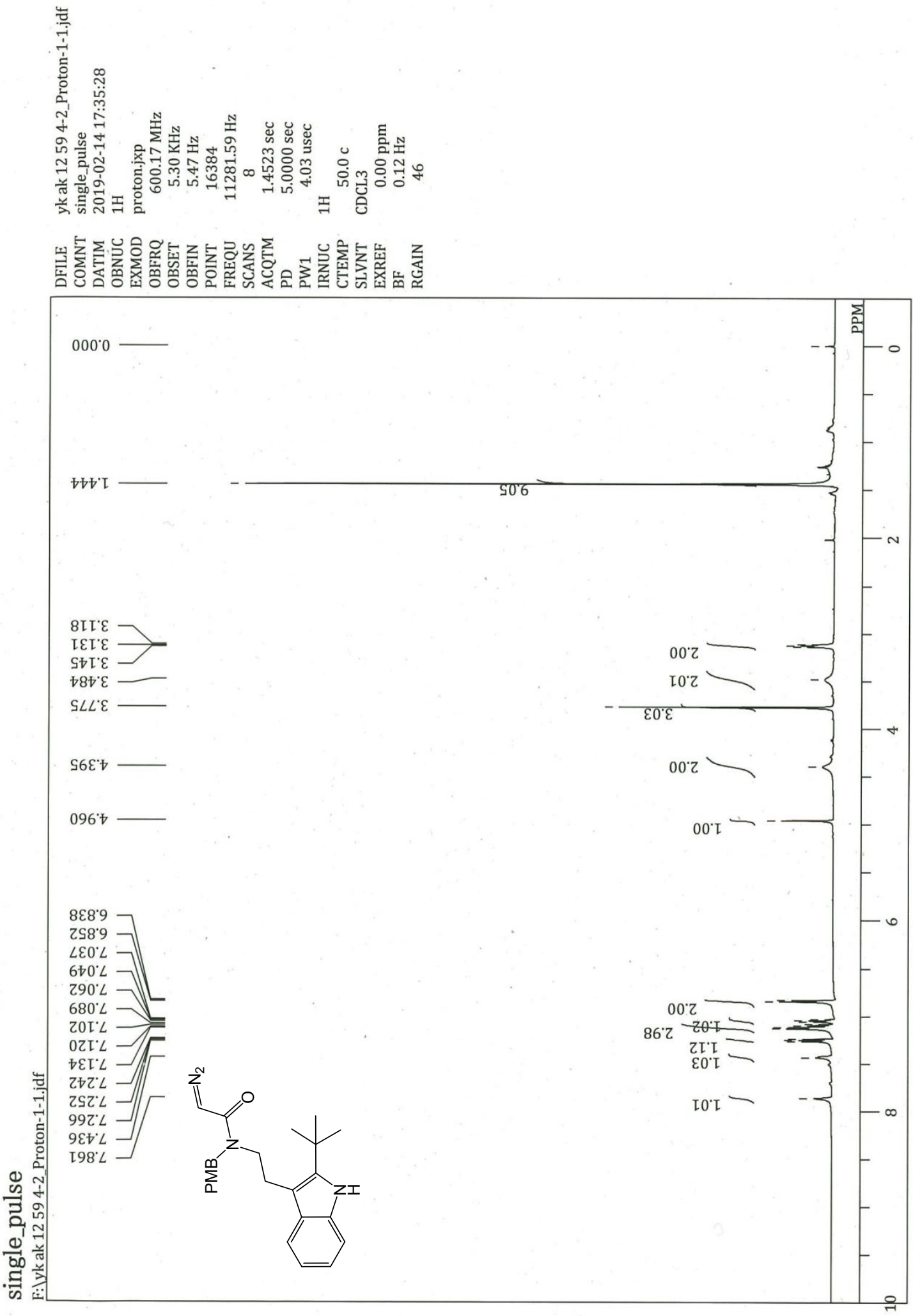
Supporting Information

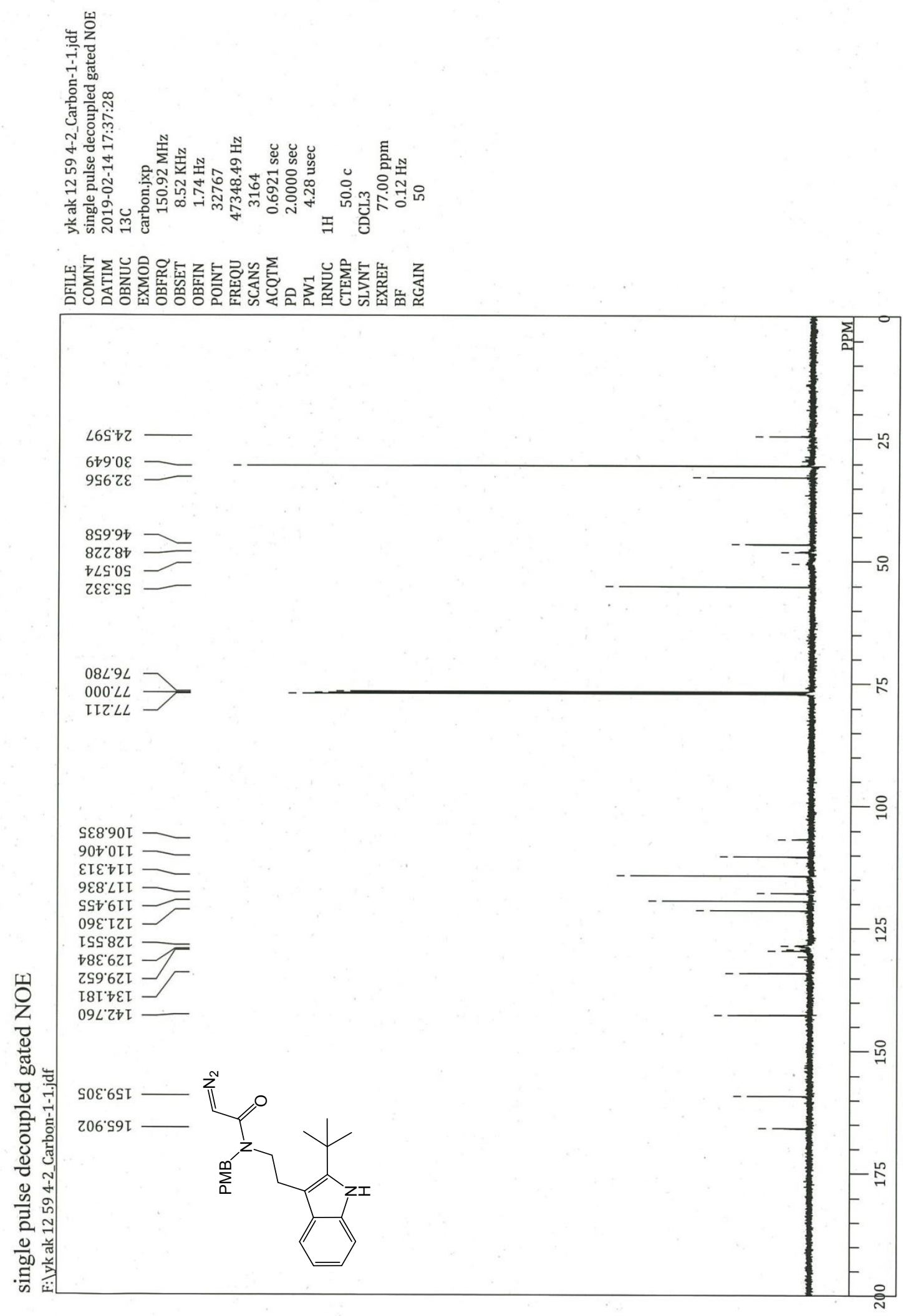


Supporting Information

$1 \mathrm{k}$

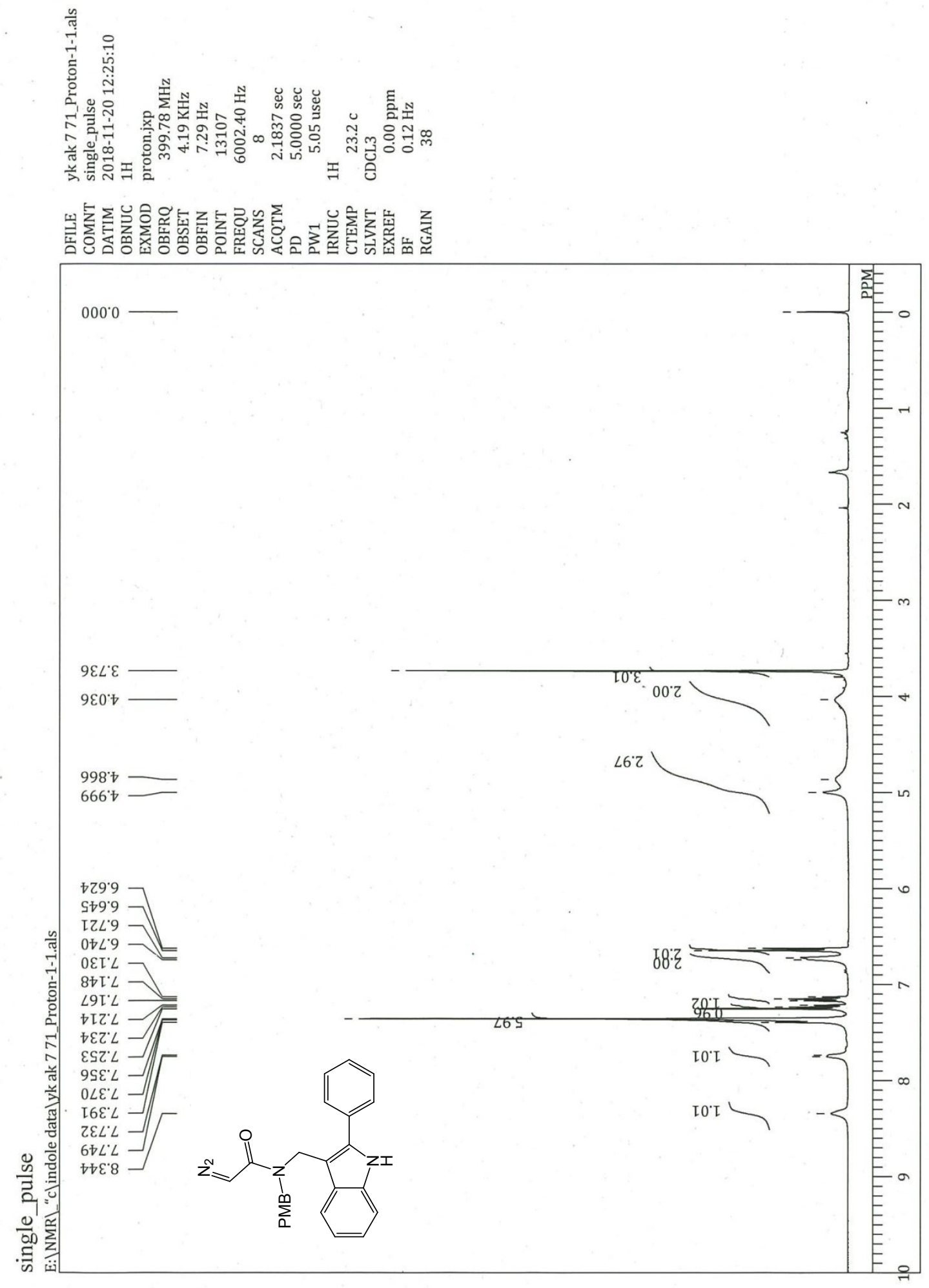


Supporting Information

$1 \mathrm{k}$

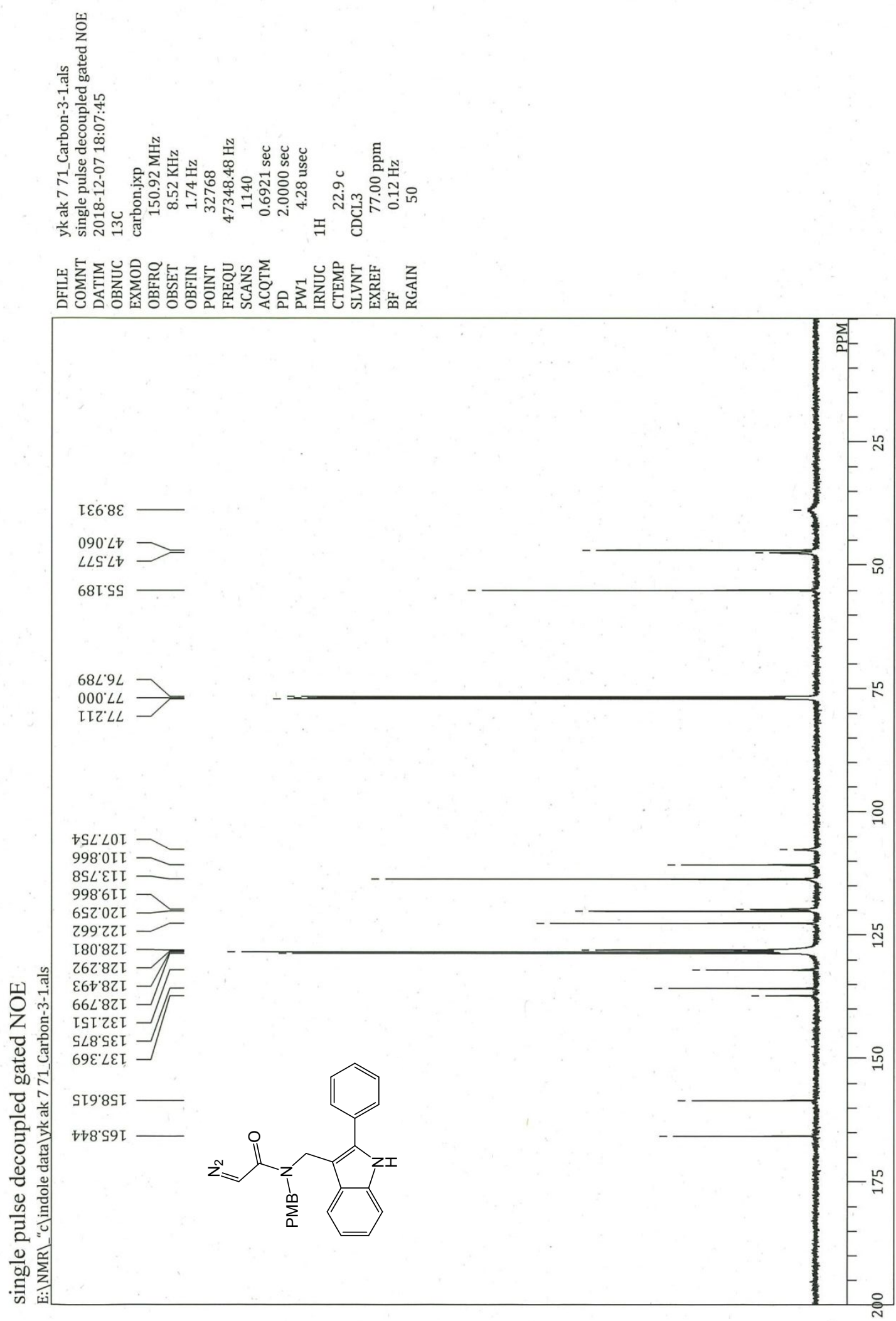


Supporting Information

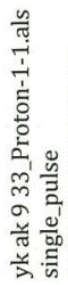

踀

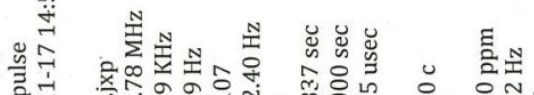

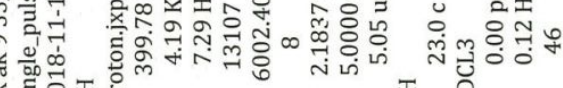

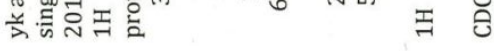

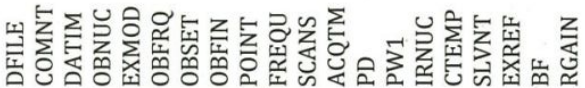

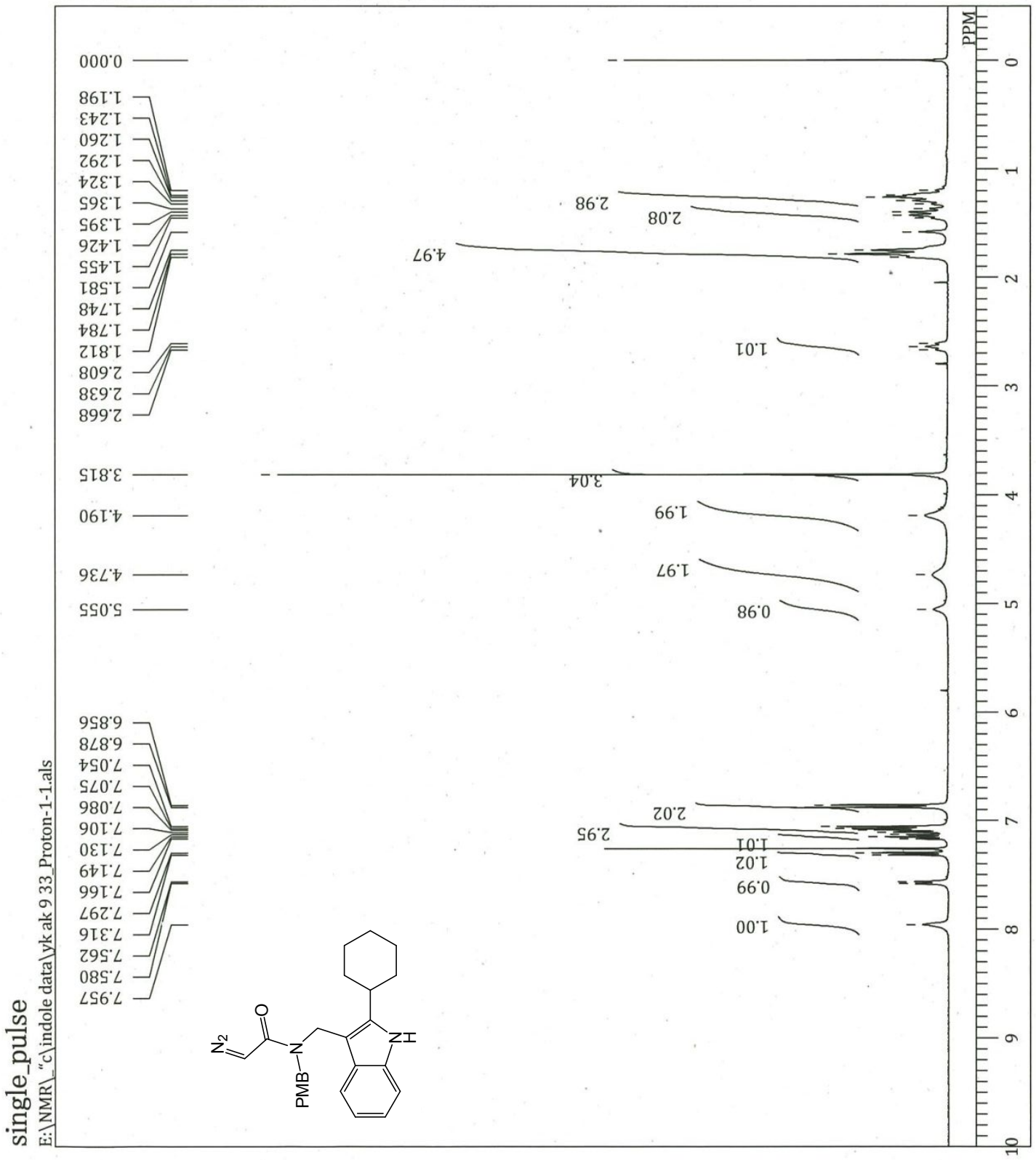


Supporting Information

11

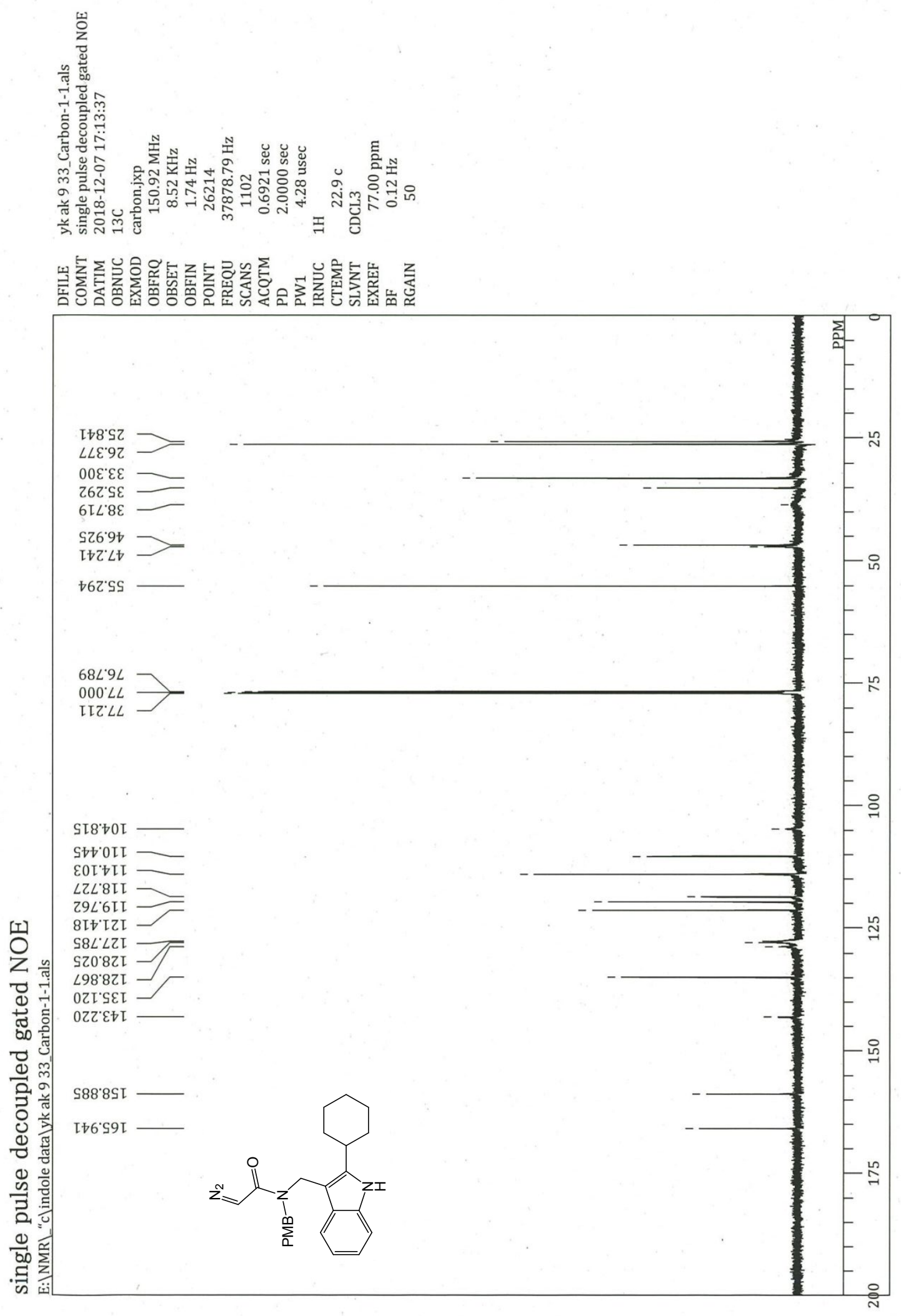


Supporting Information

$1 \mathrm{~m}$
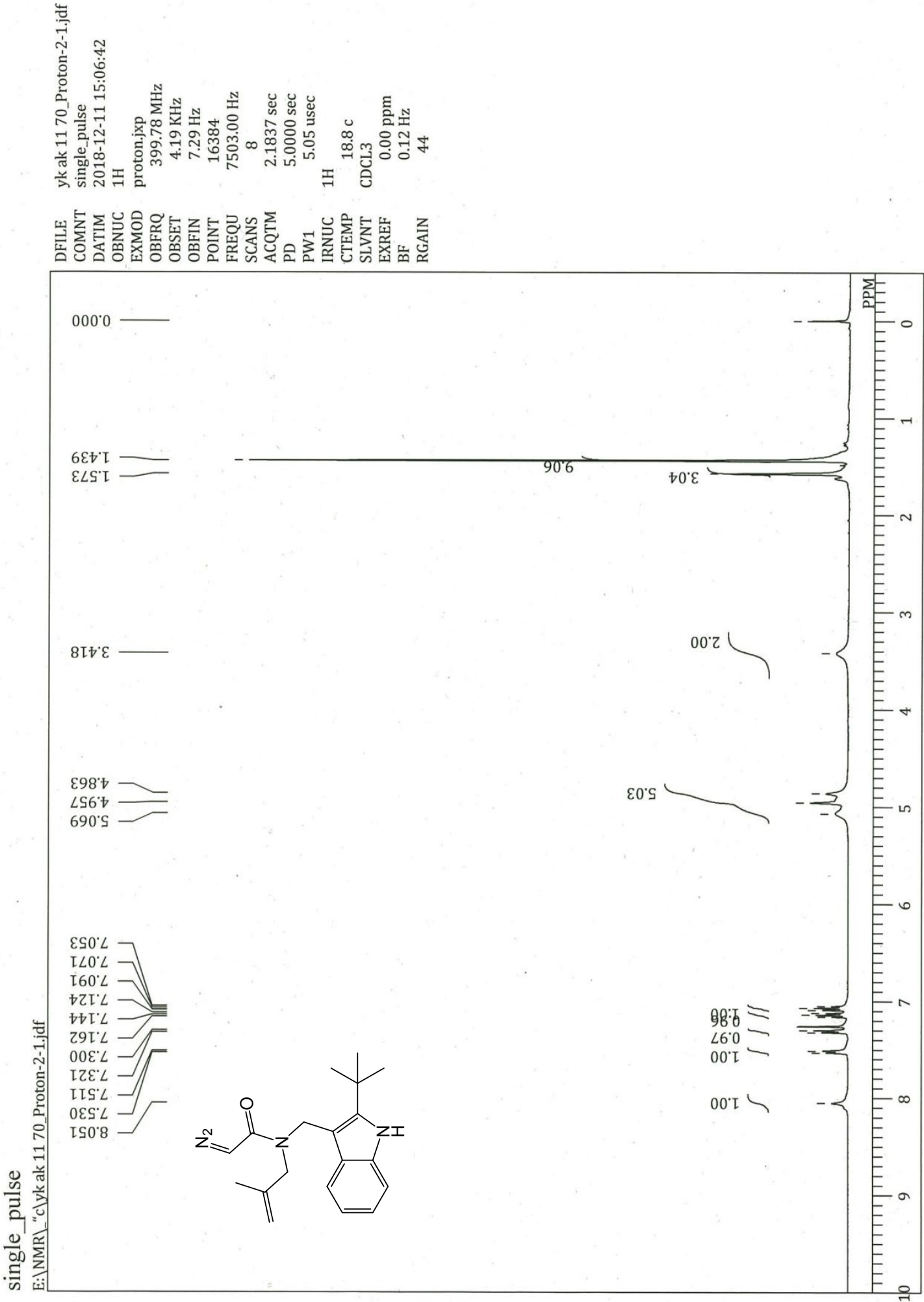
Supporting Information

$1 \mathrm{~m}$

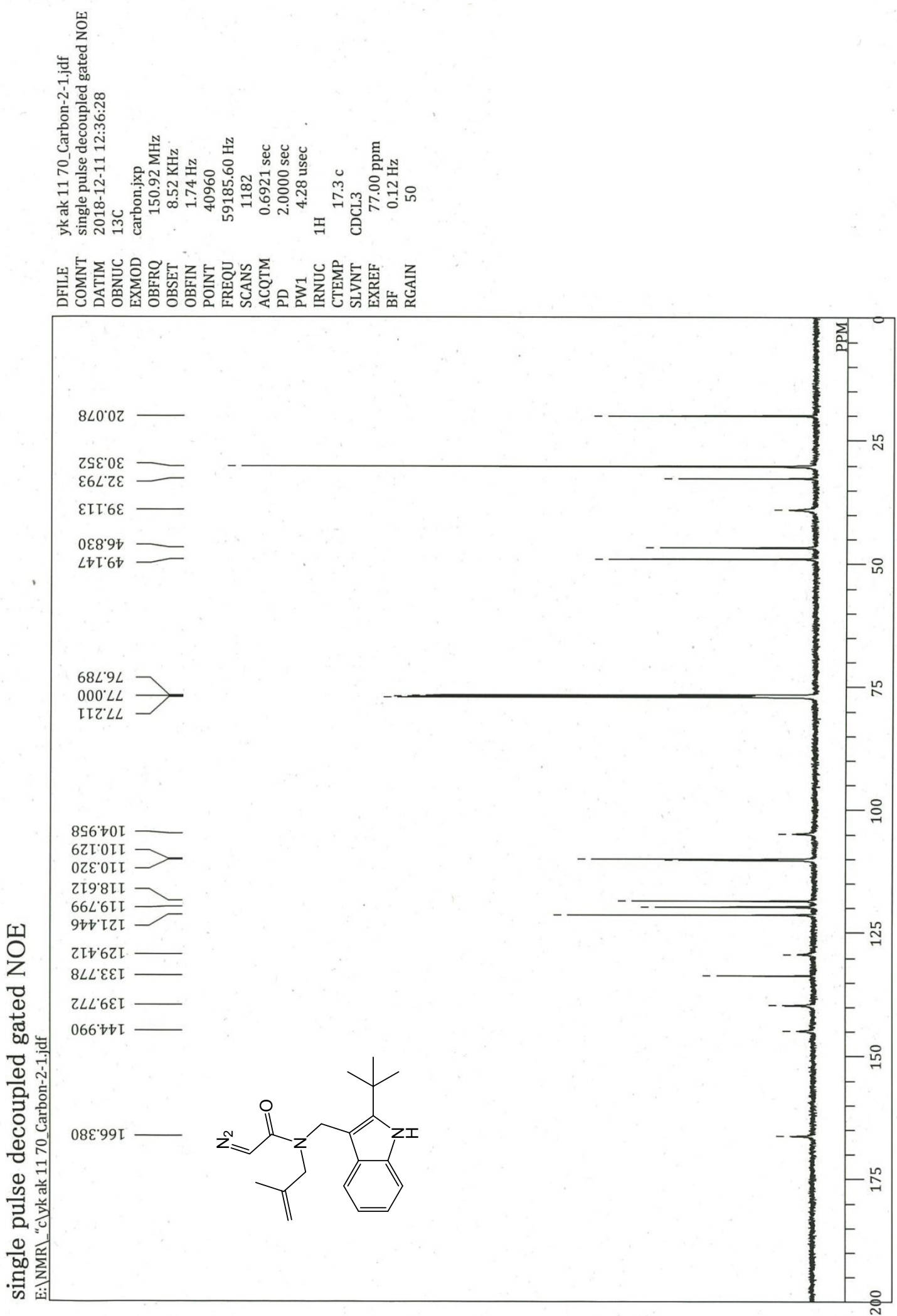


Supporting Information

1n

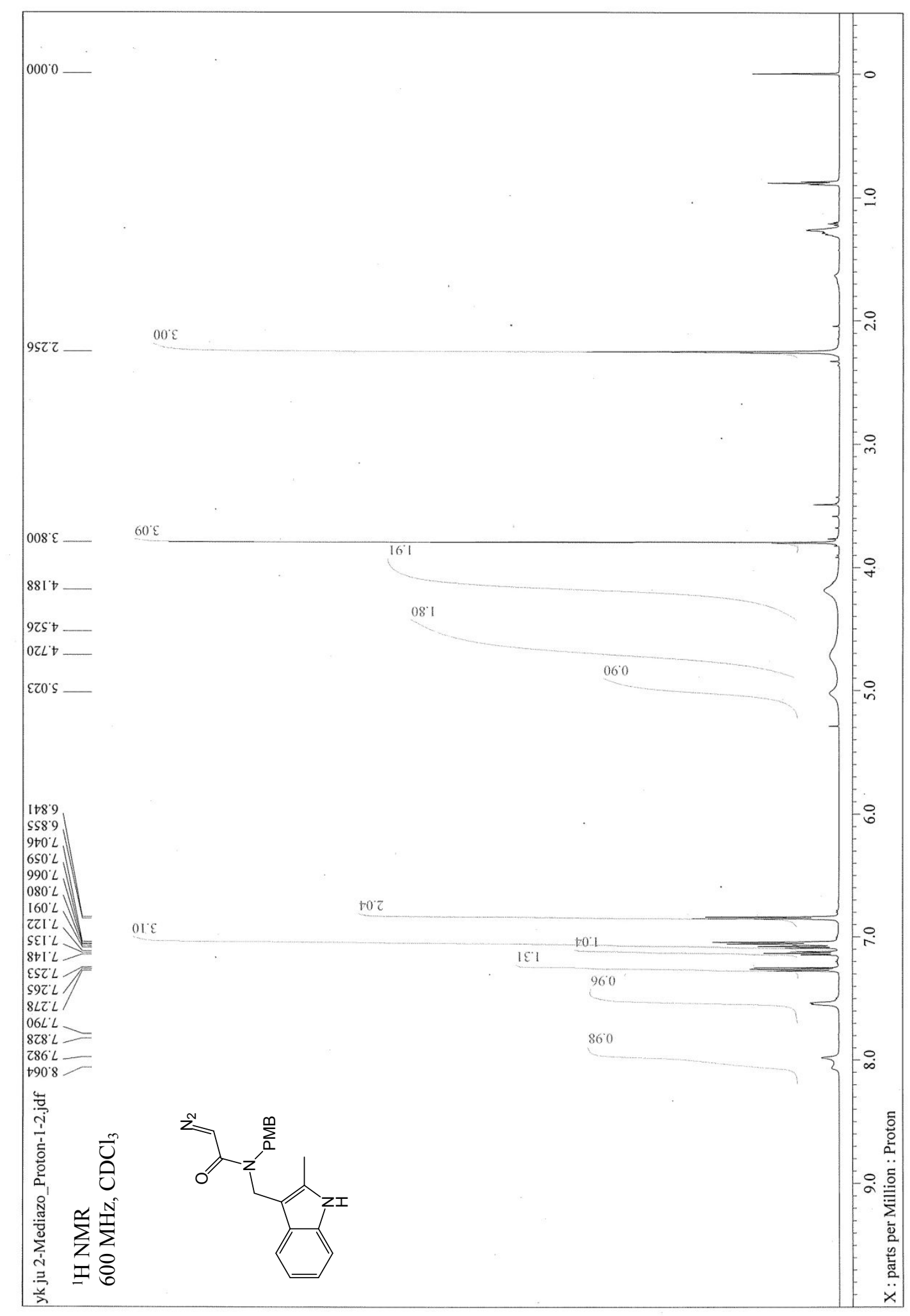


Supporting Information

$1 n$

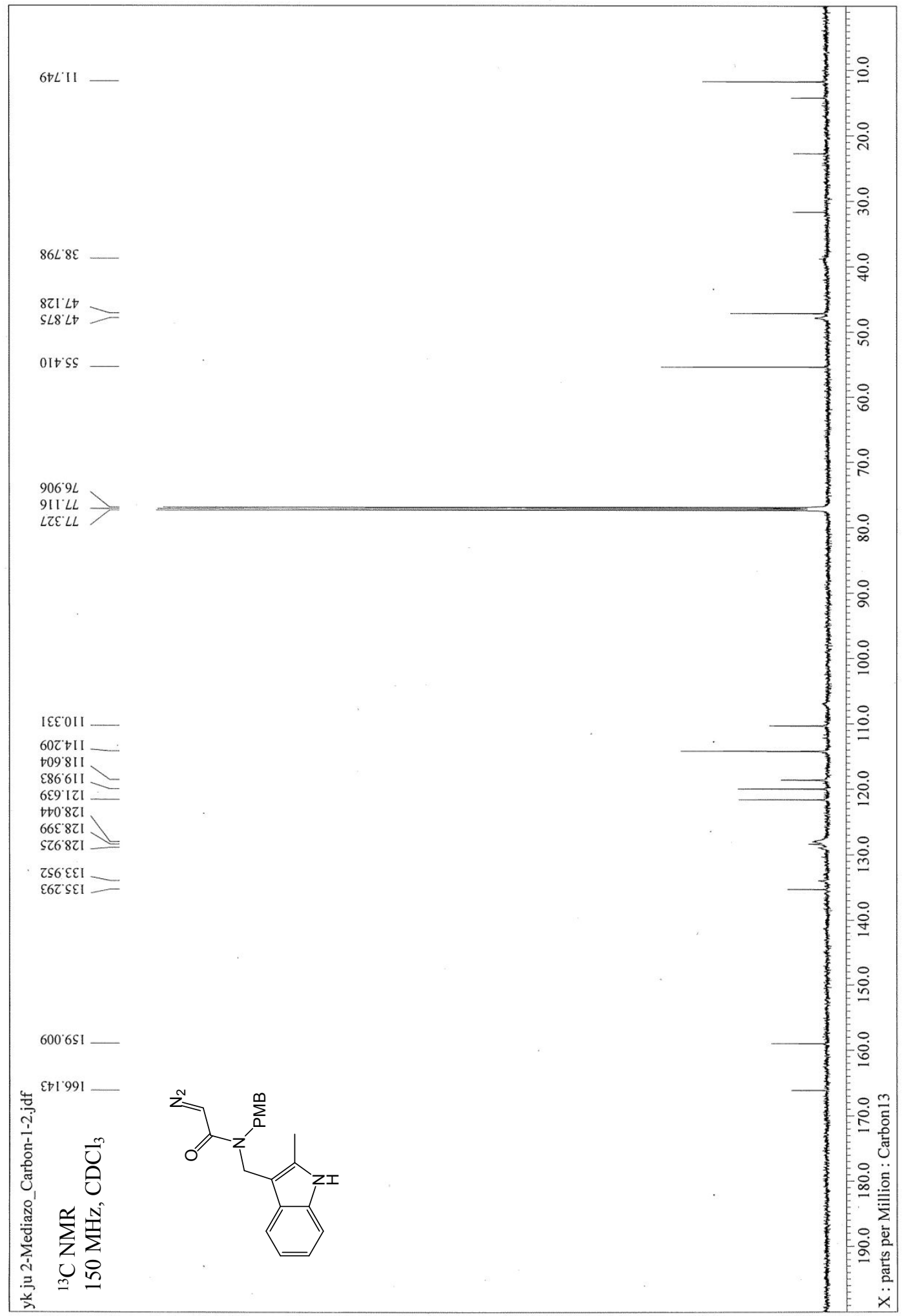


Supporting Information

10

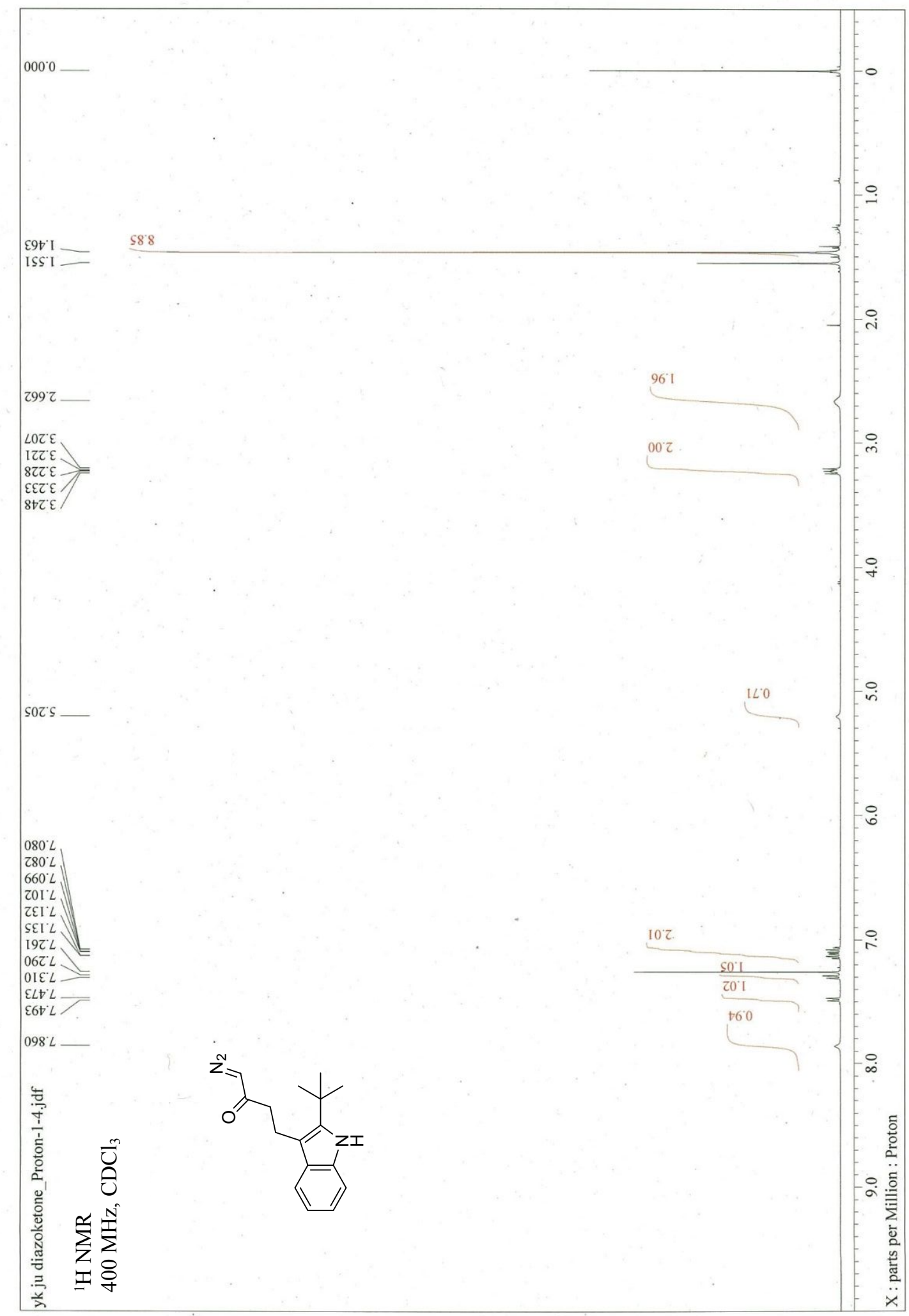


Supporting Information

10

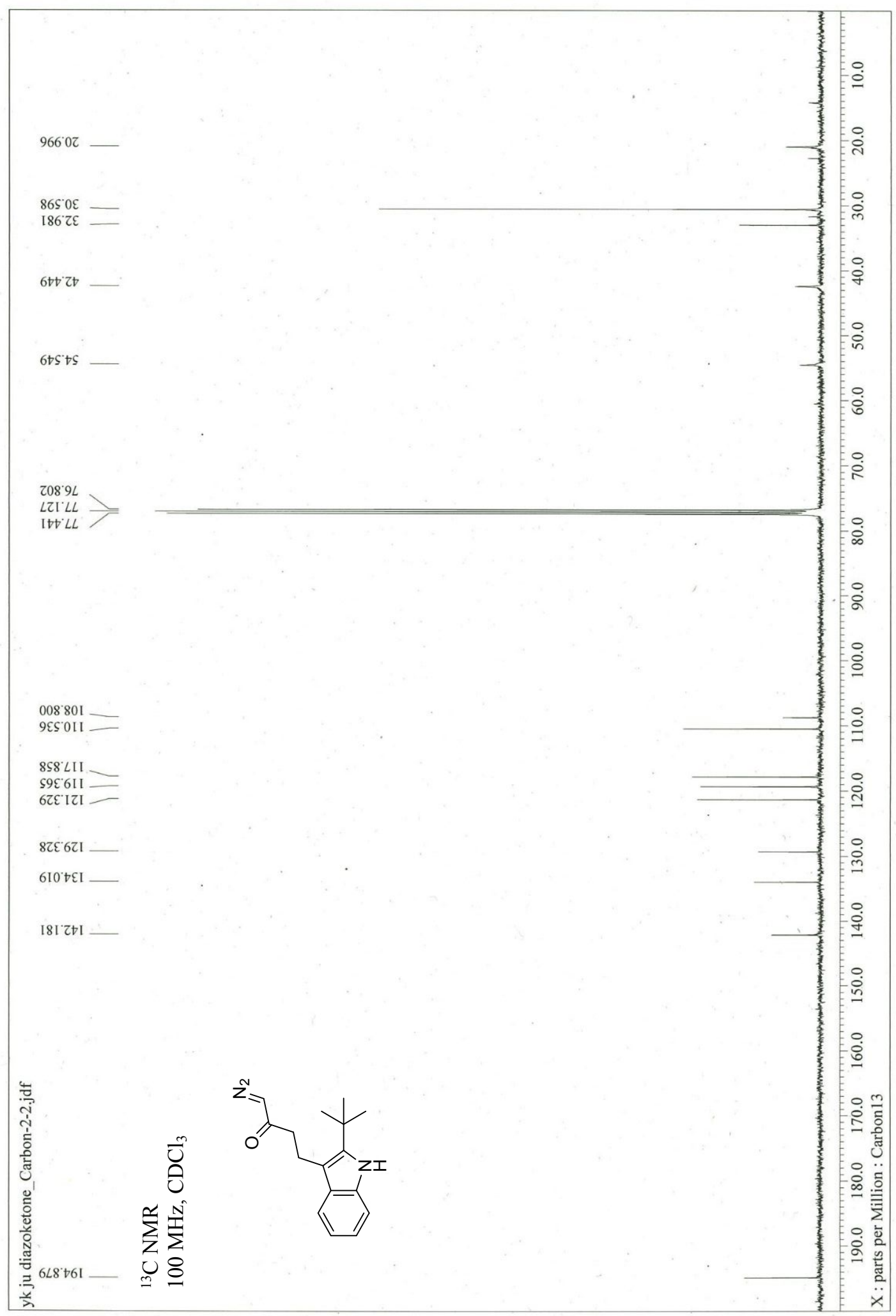


Supporting Information

$3 a$
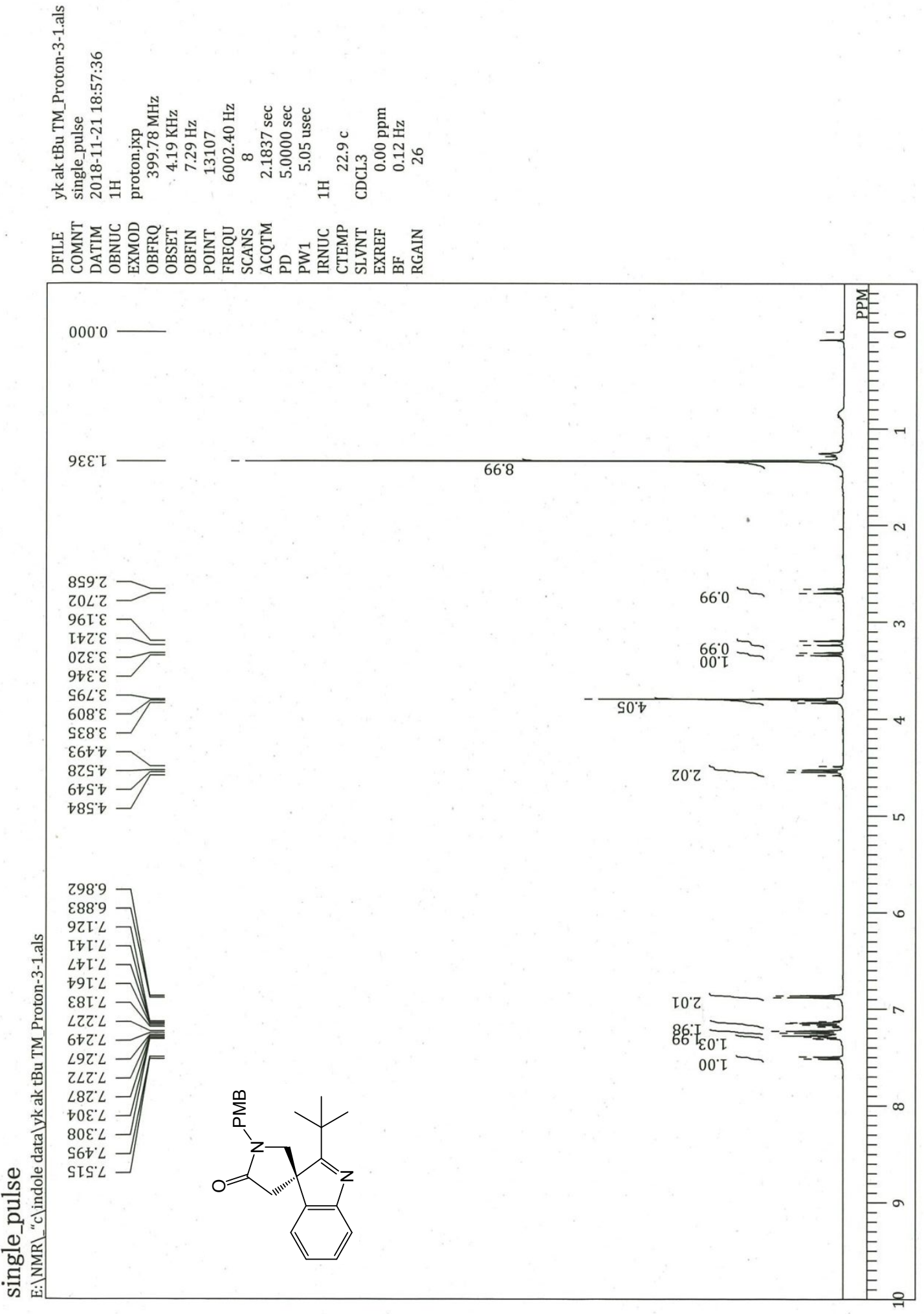
Supporting Information

$3 a$

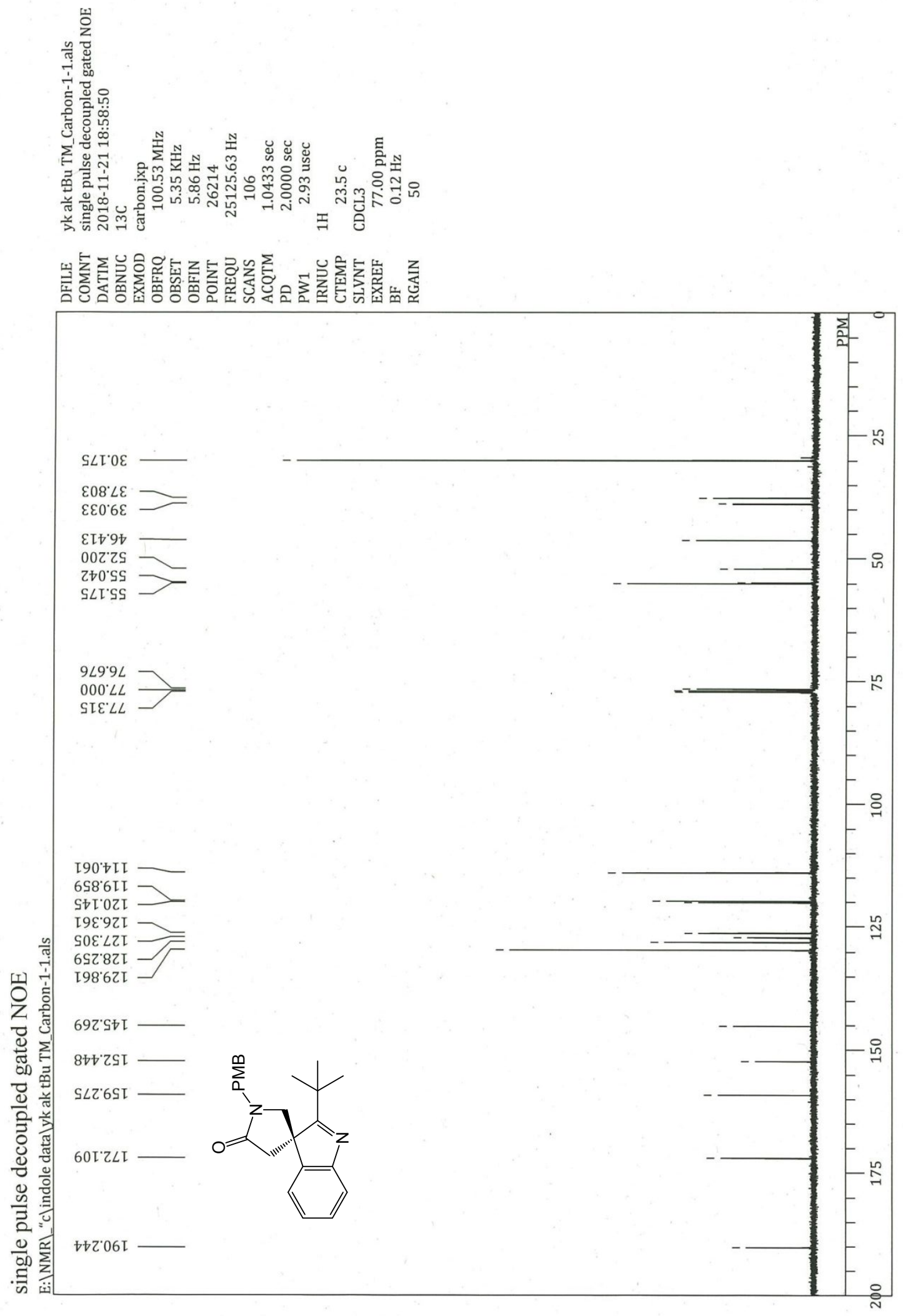


Supporting Information

$3 b$

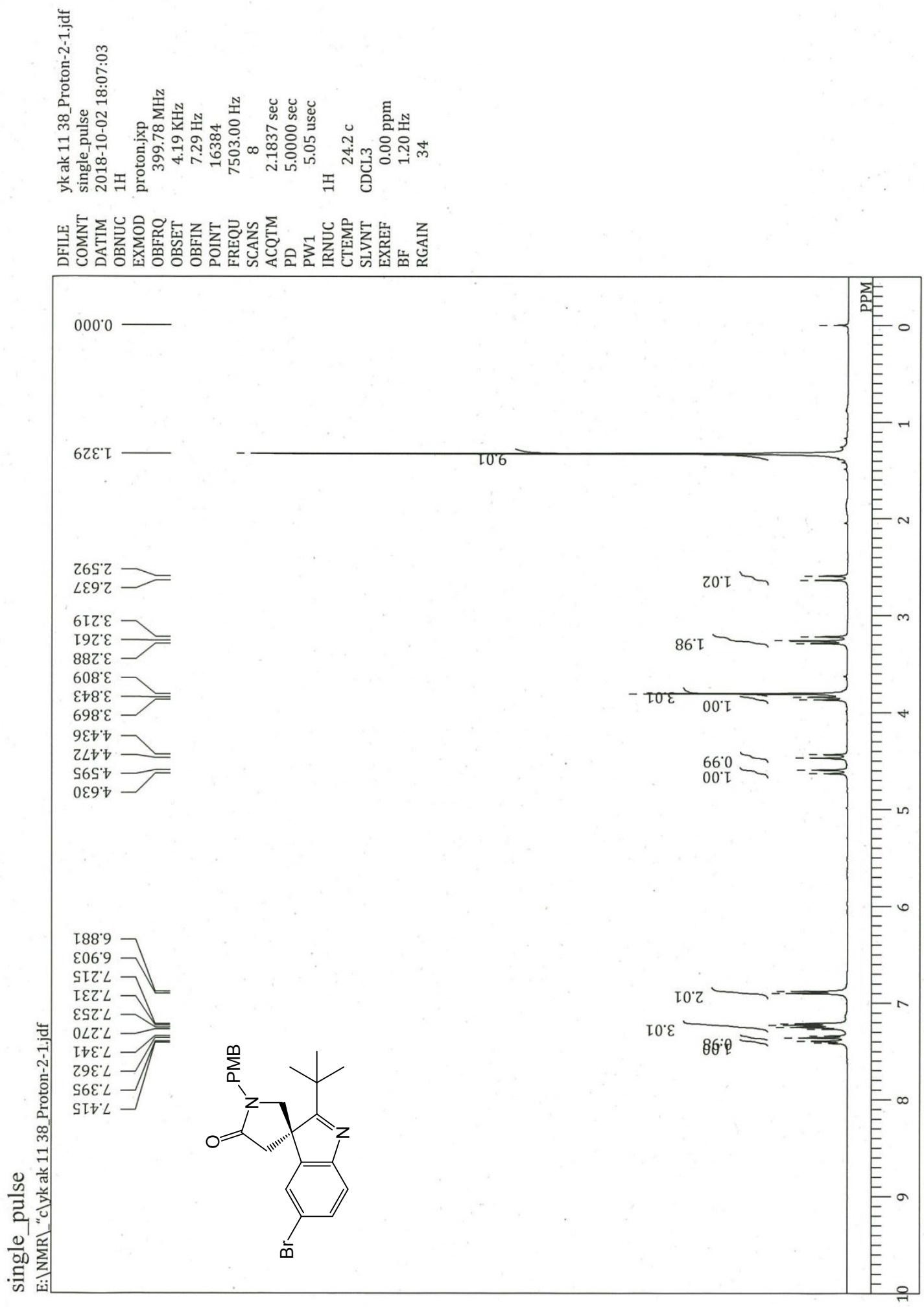


Supporting Information

$3 b$

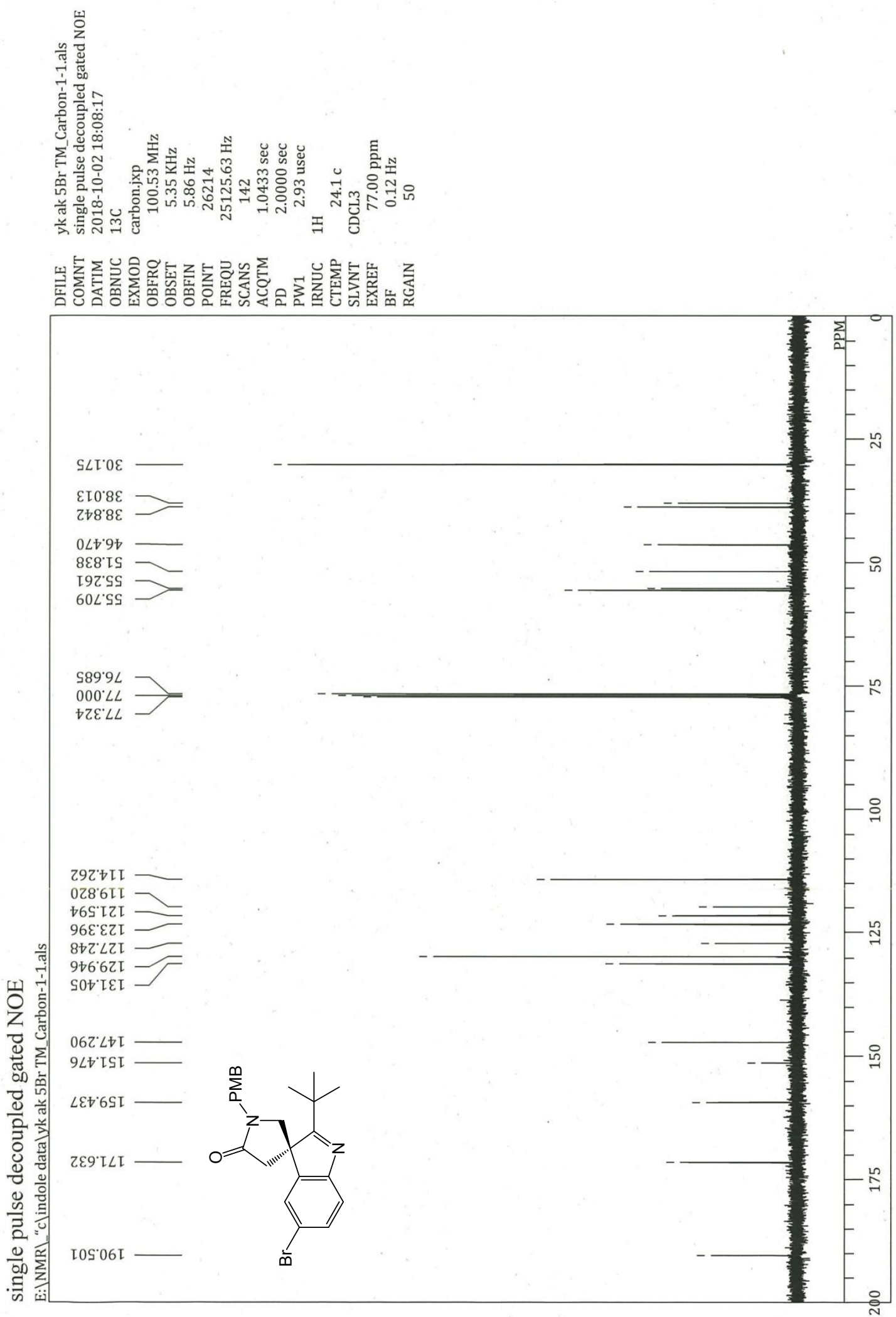


Supporting Information

$3 c$
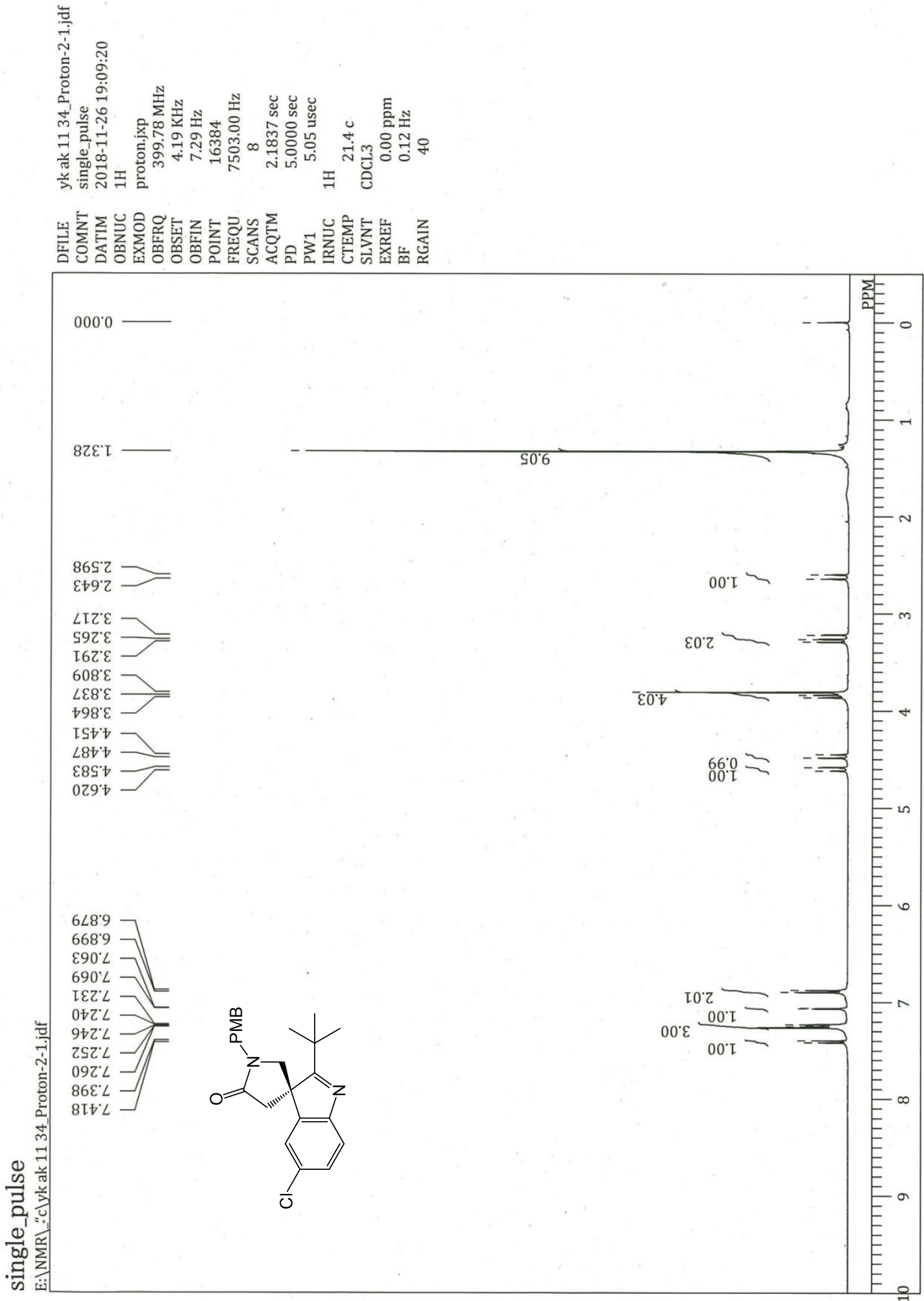
Supporting Information

$3 c$

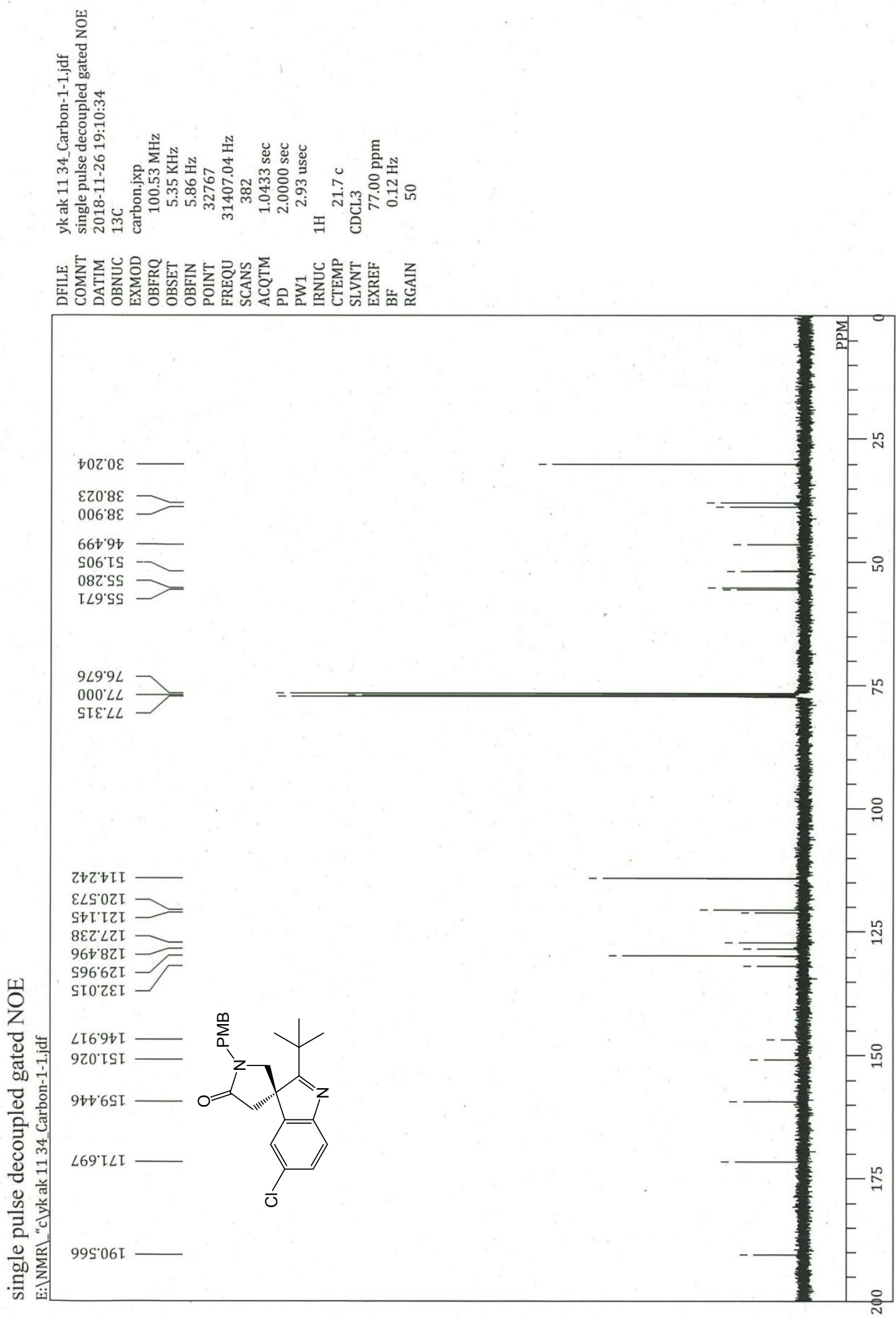


Supporting Information

$3 d$
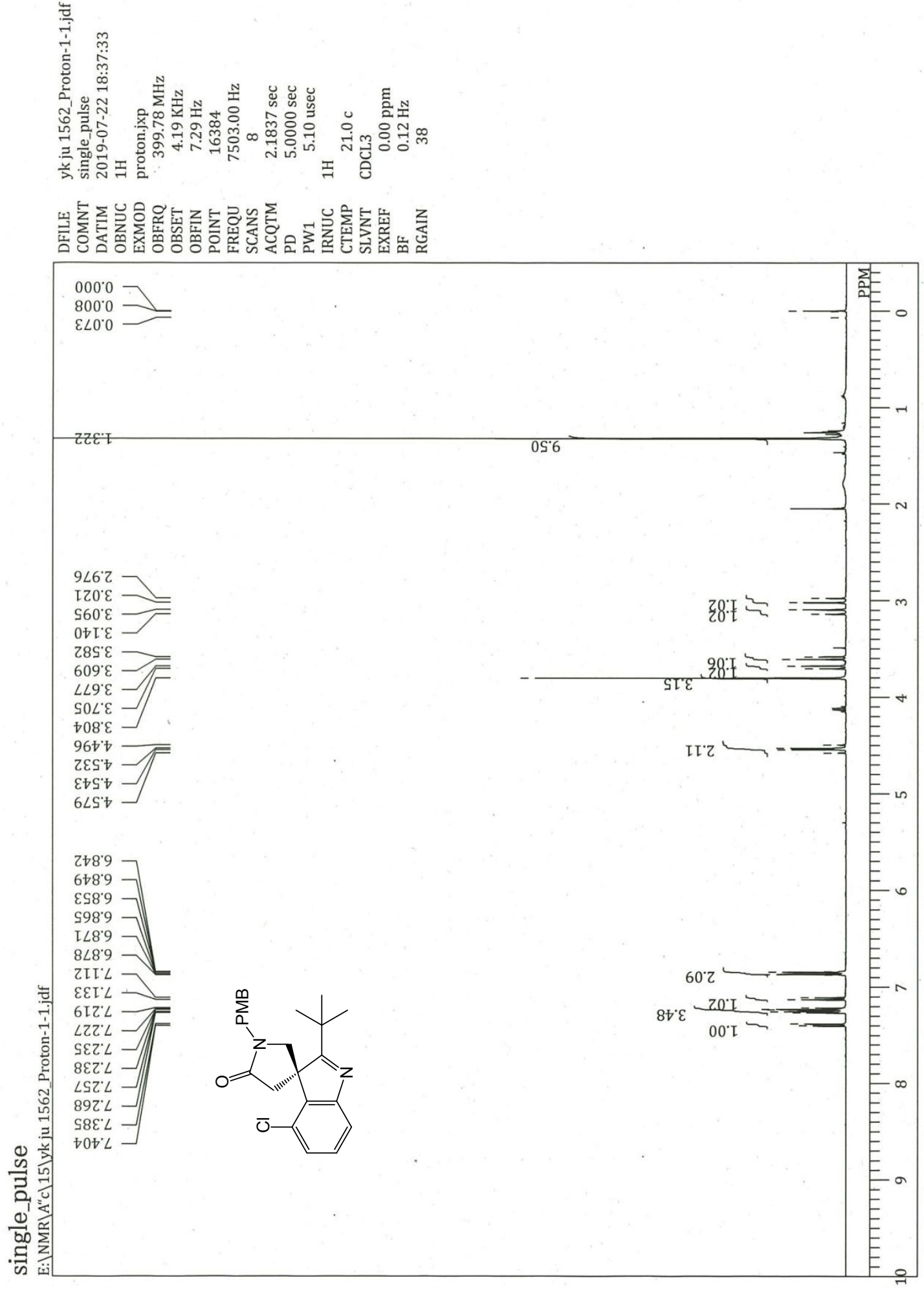
Supporting Information

$3 d$

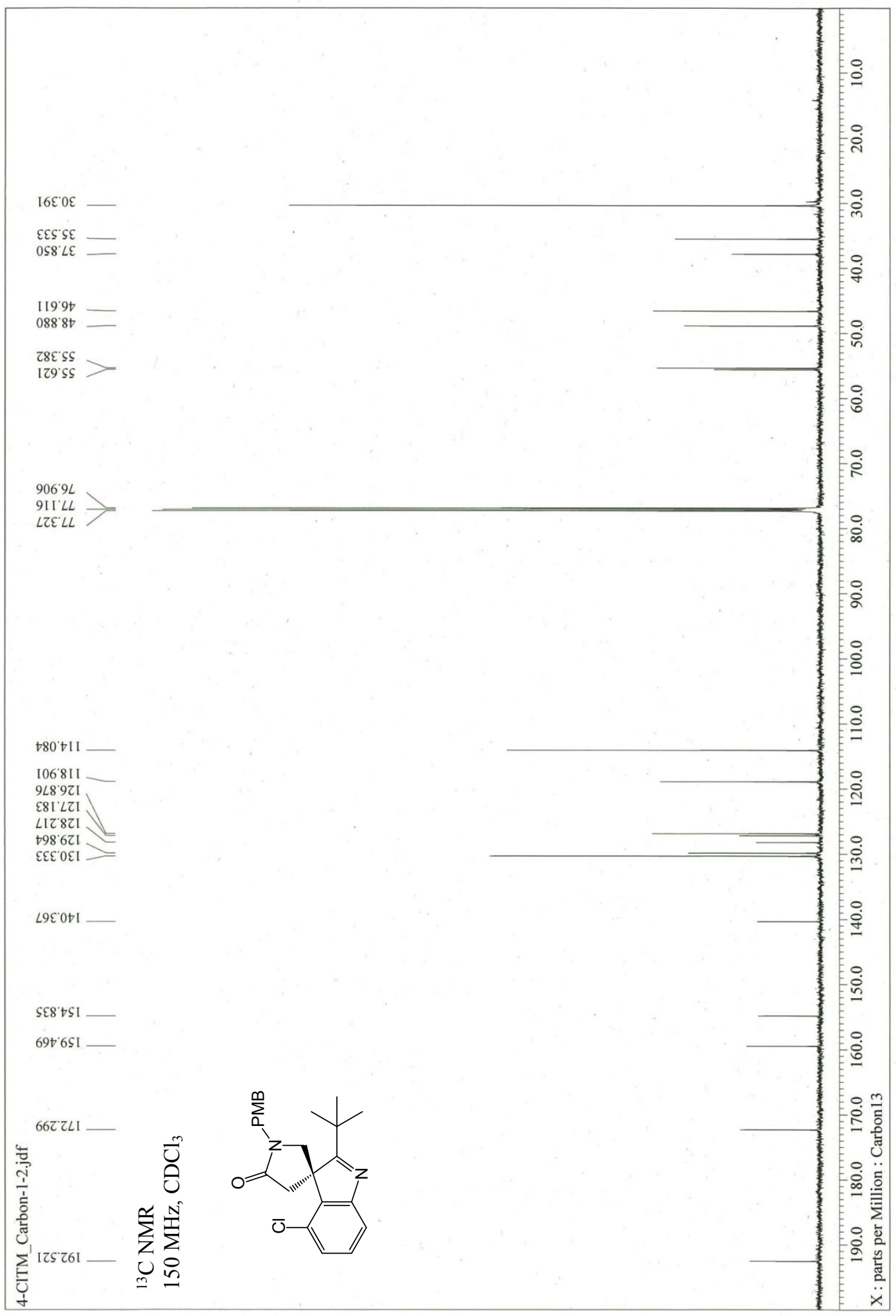


Supporting Information

$3 e$

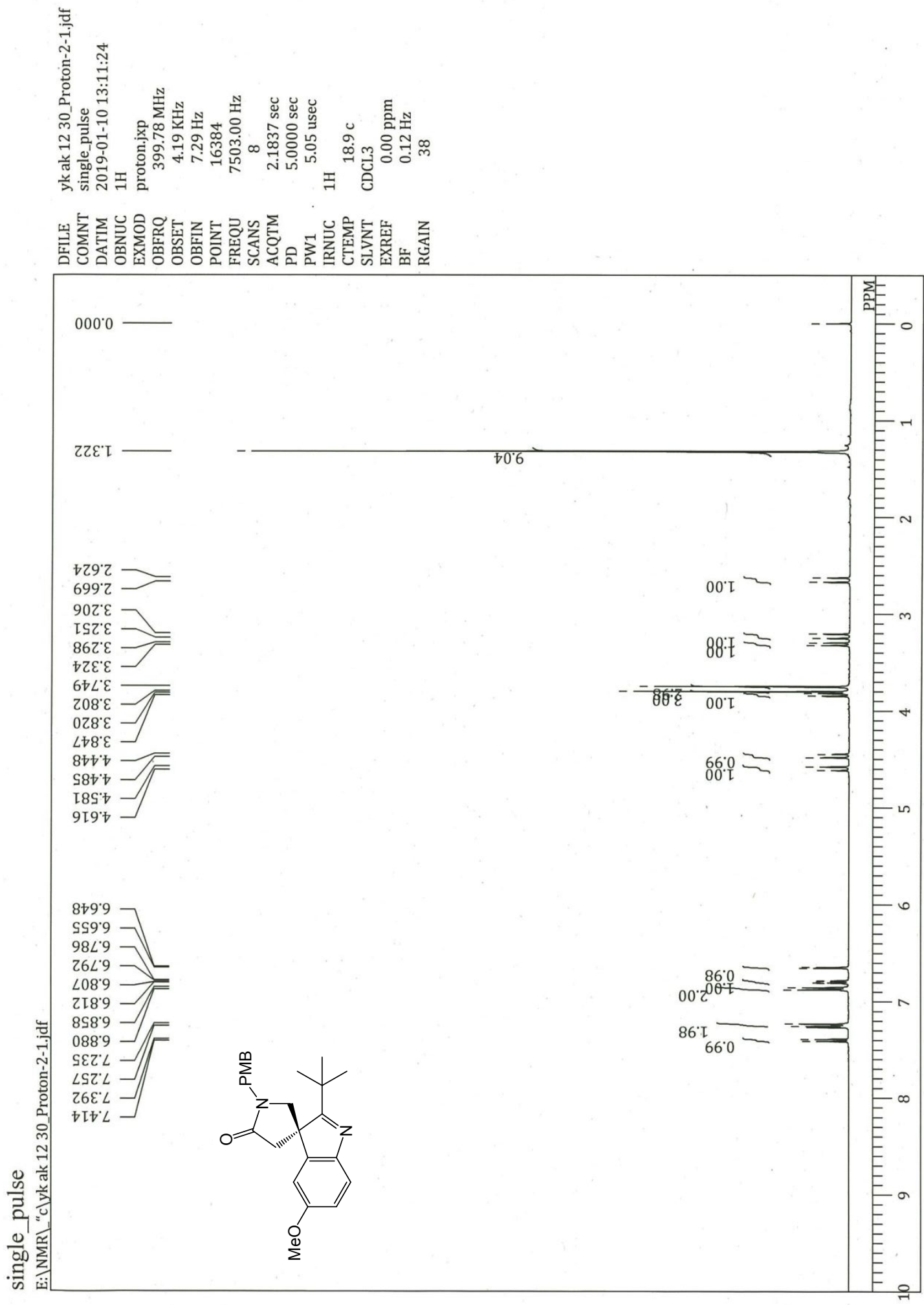


Supporting Information

$3 e$

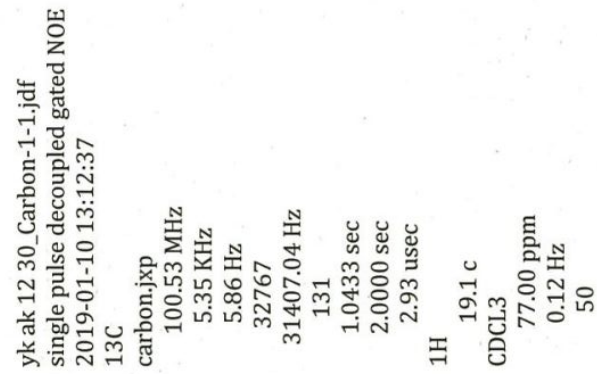

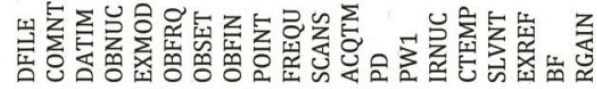

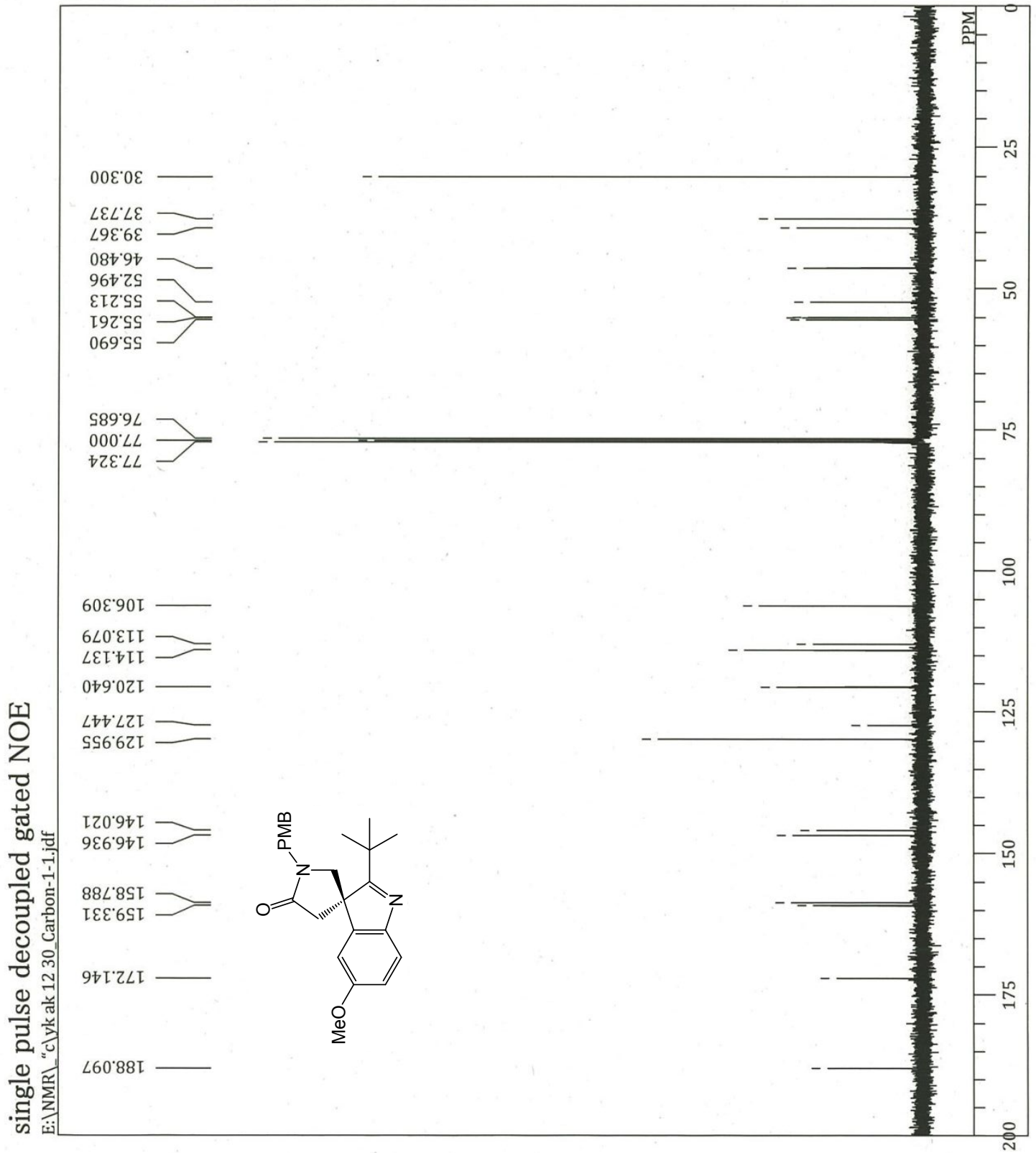


Supporting Information

$3 f$
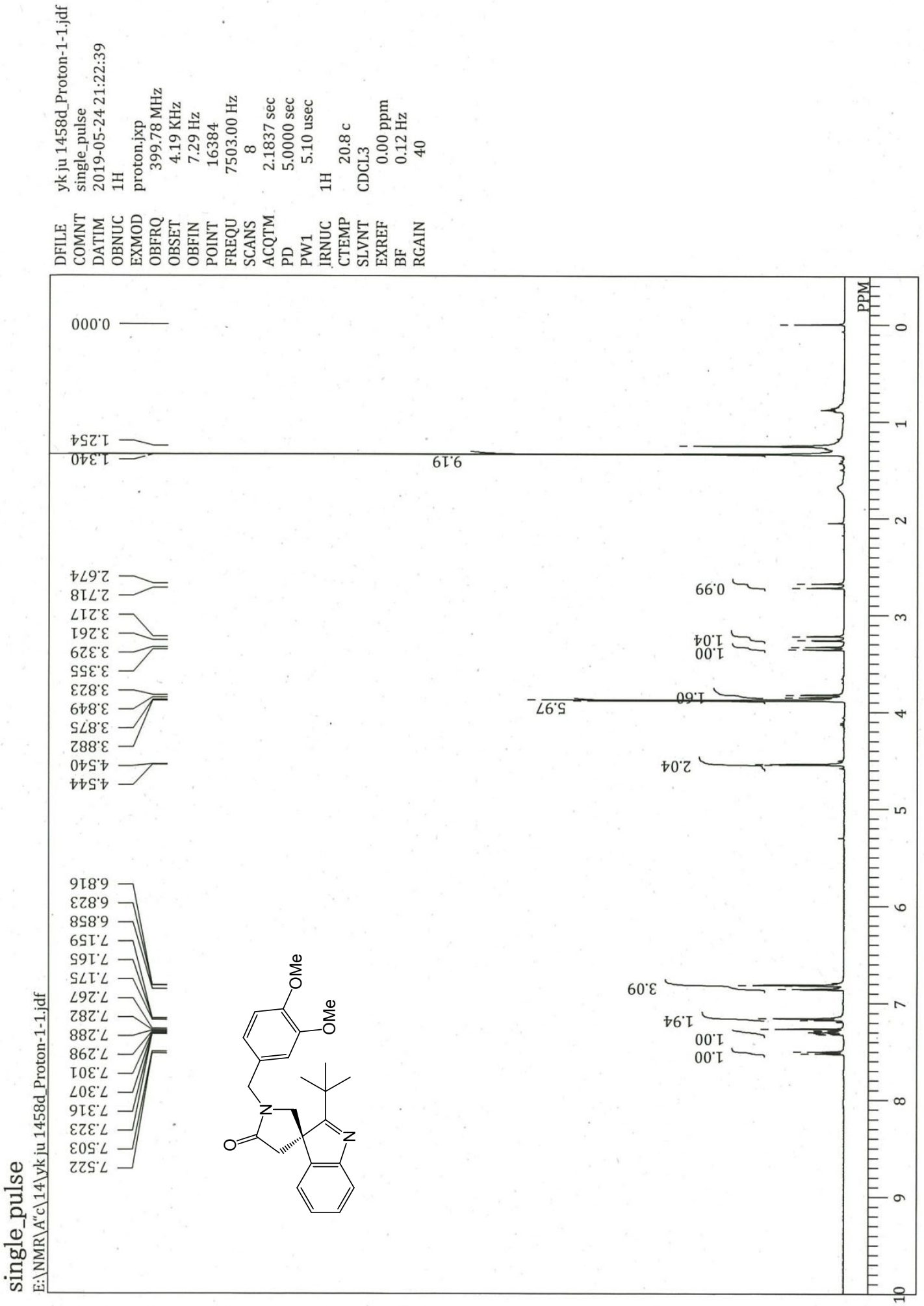
Supporting Information

$3 f$
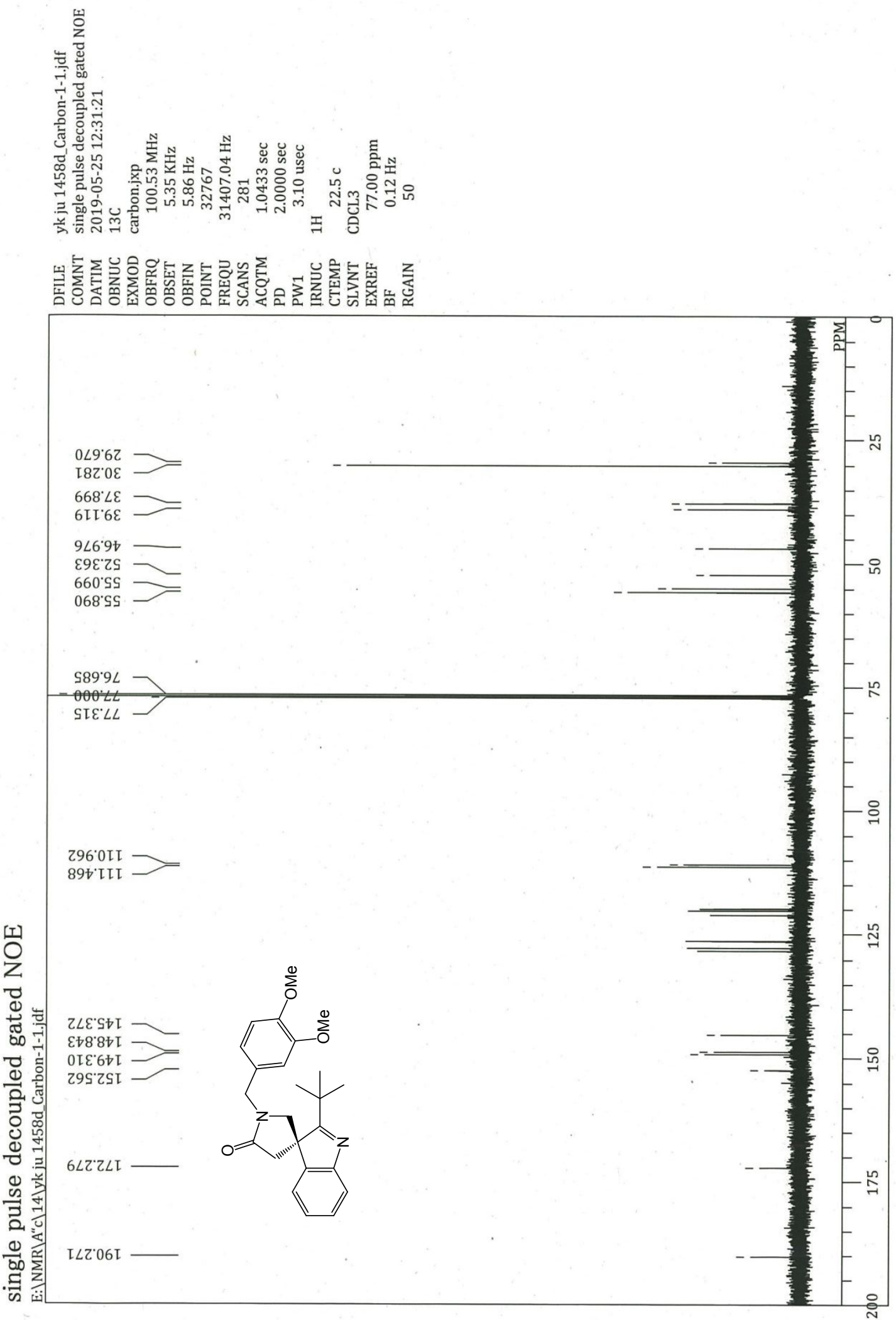
Supporting Information

$3 g$
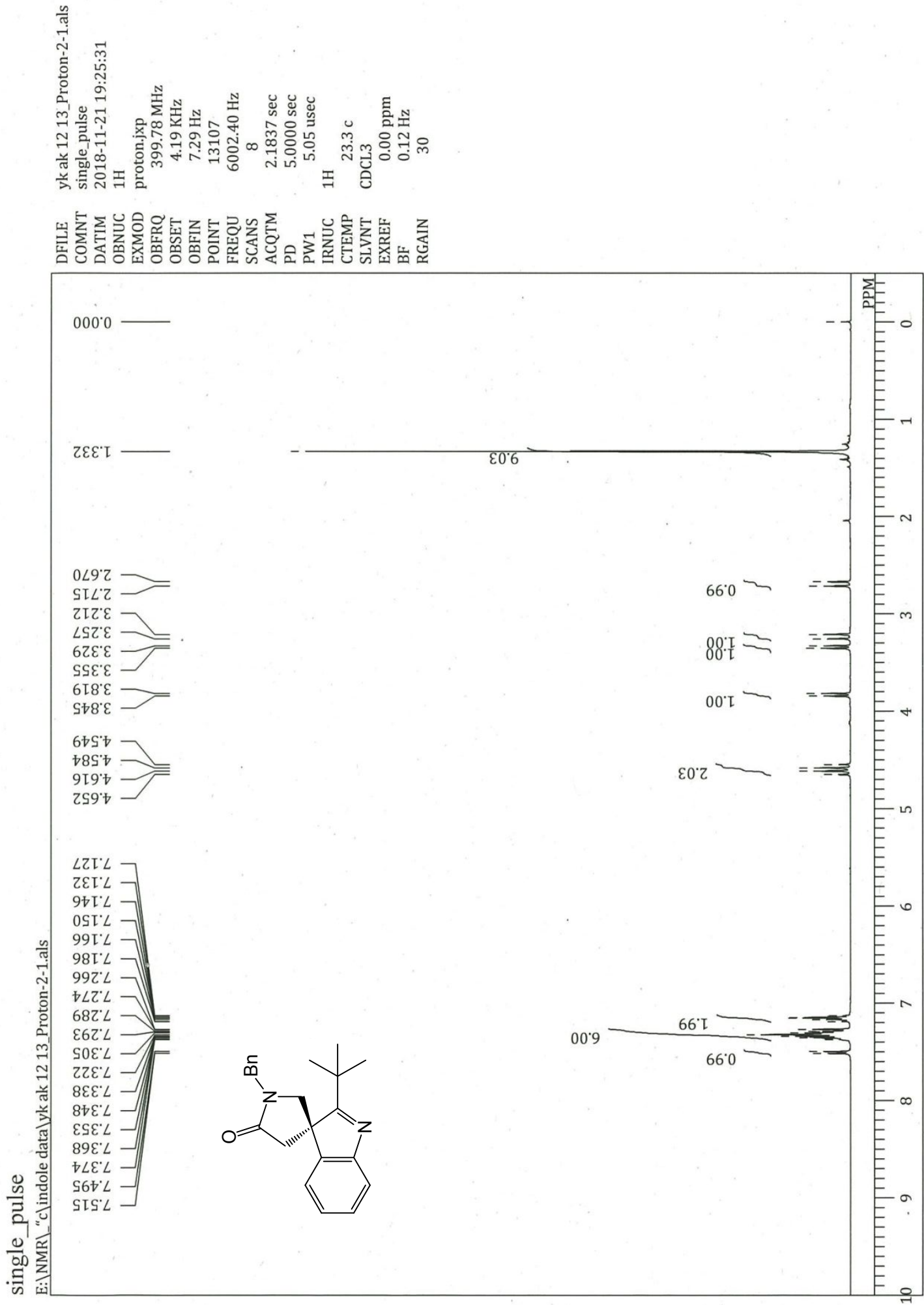
Supporting Information

$3 g$

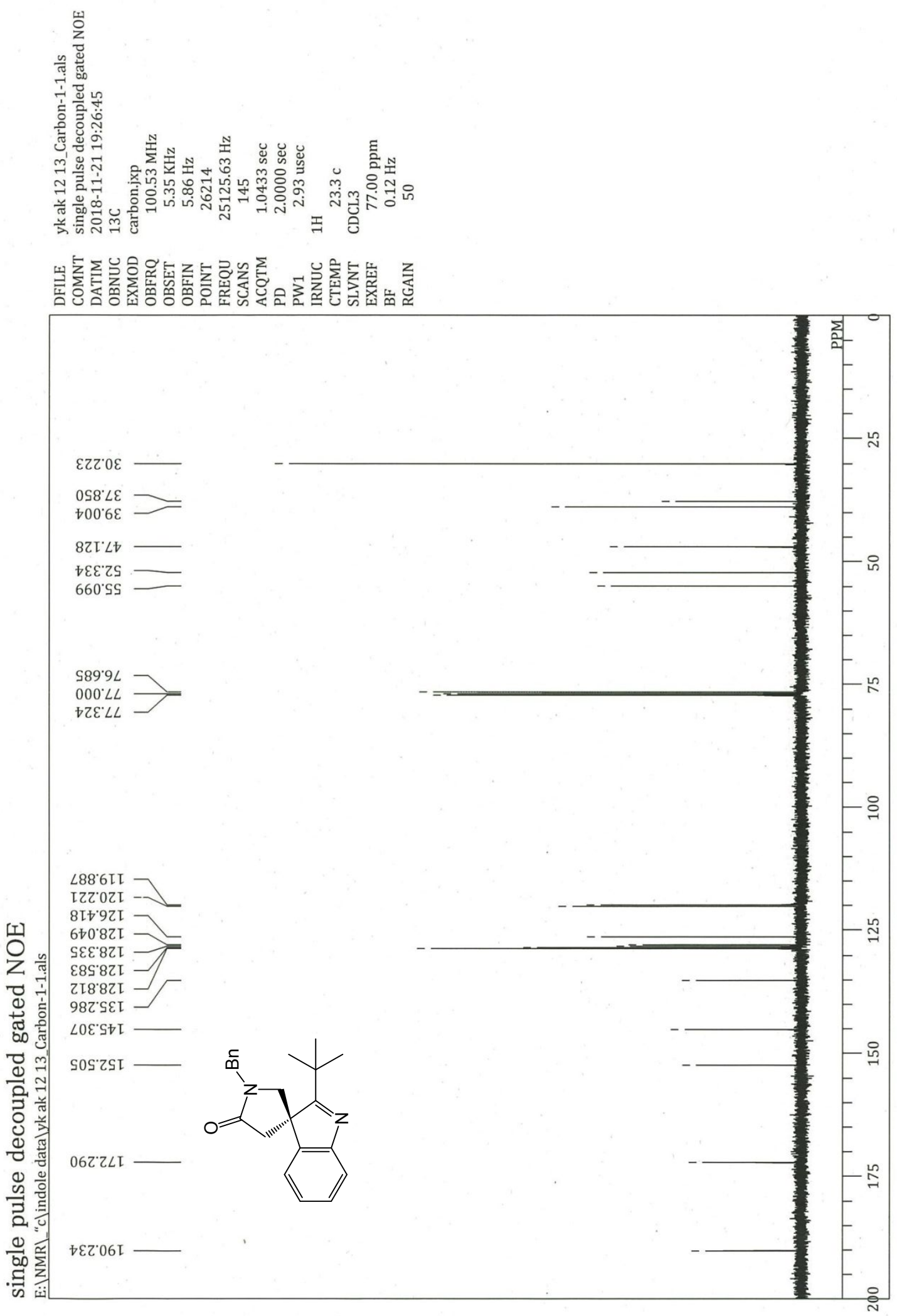


Supporting Information

$3 \mathrm{~h}$
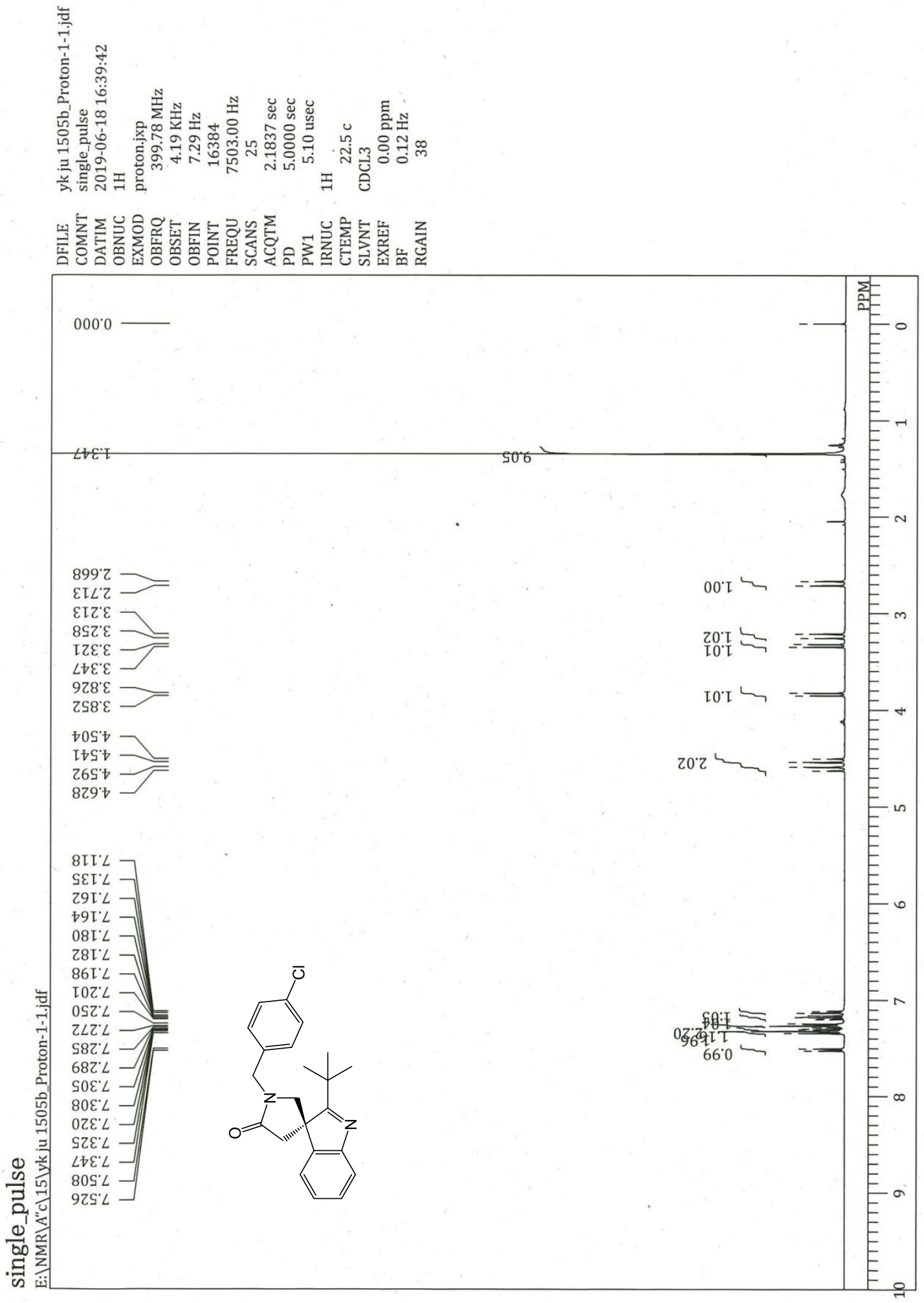
Supporting Information

$3 \mathrm{~h}$

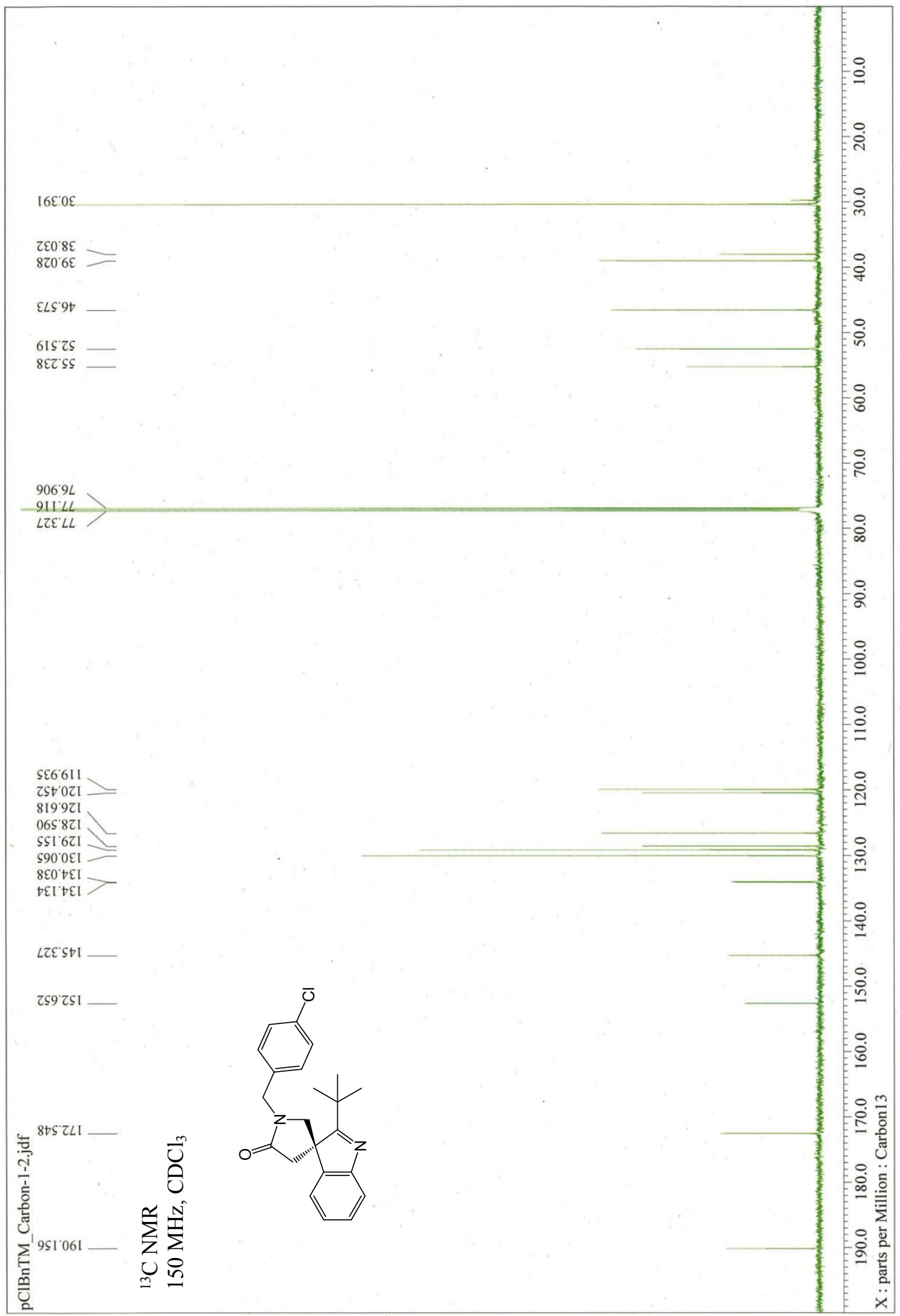


Supporting Information

$3 \mathbf{i}$

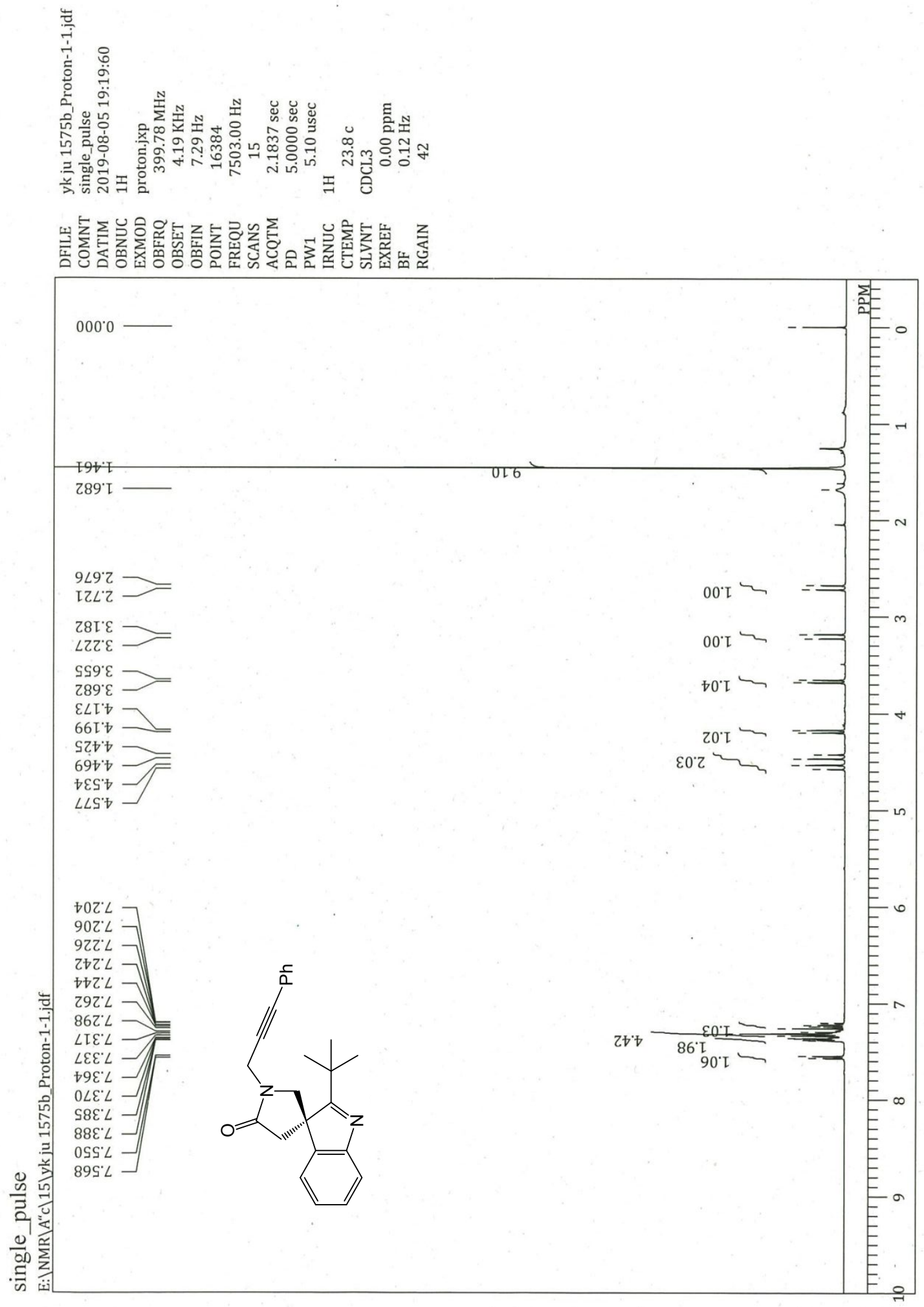


Supporting Information

$3 \mathbf{i}$

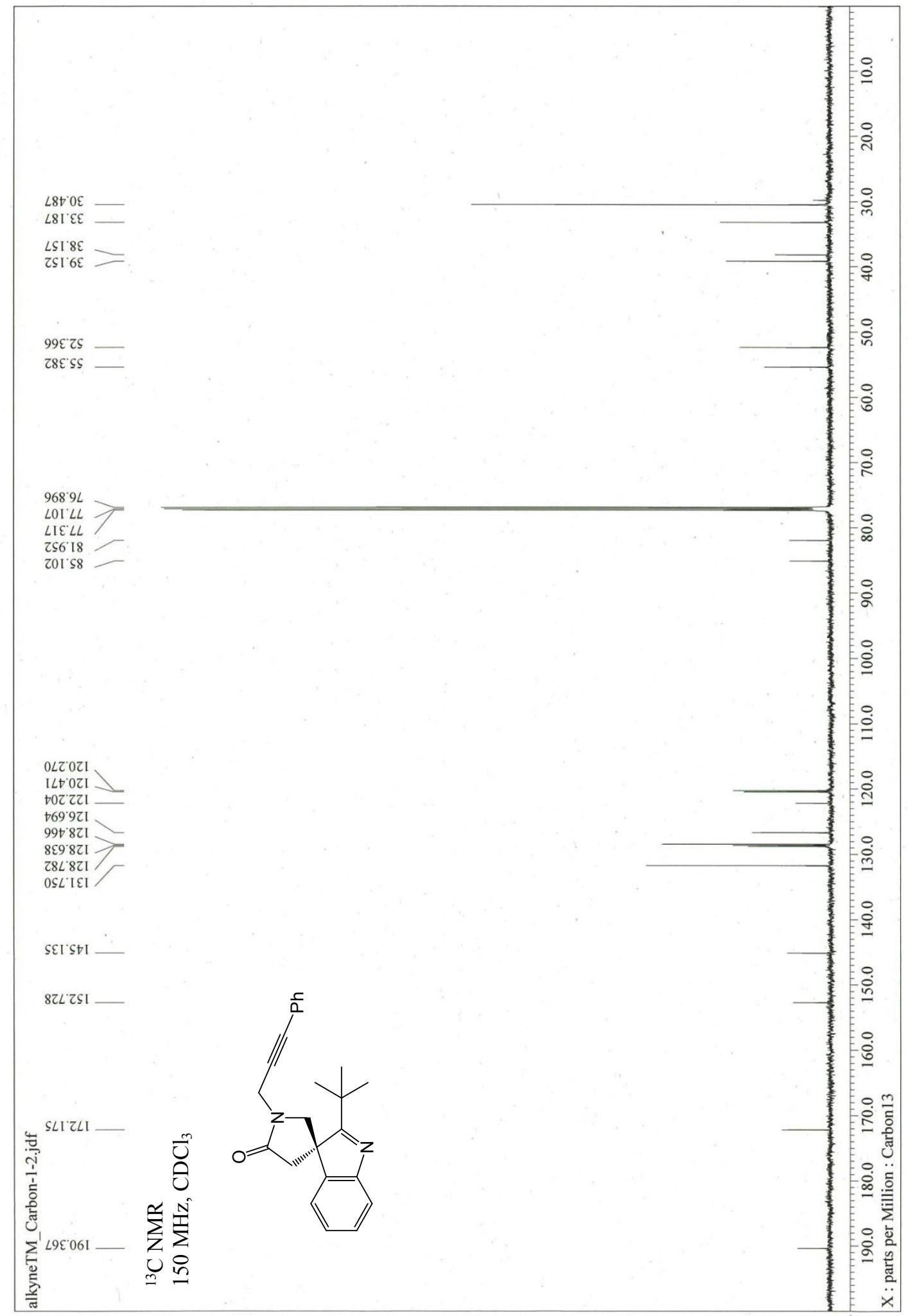


Supporting Information

3j
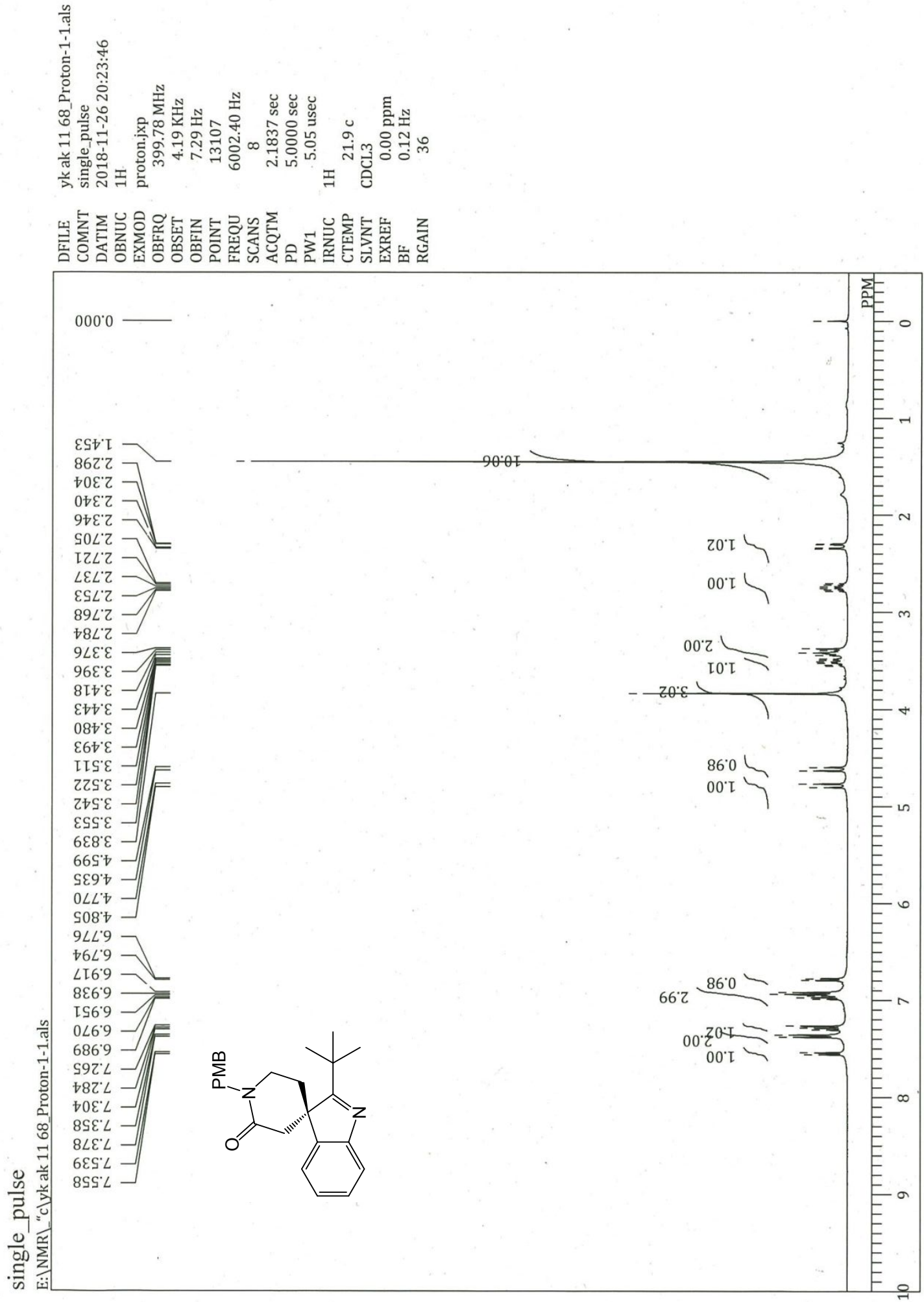
Supporting Information

3j

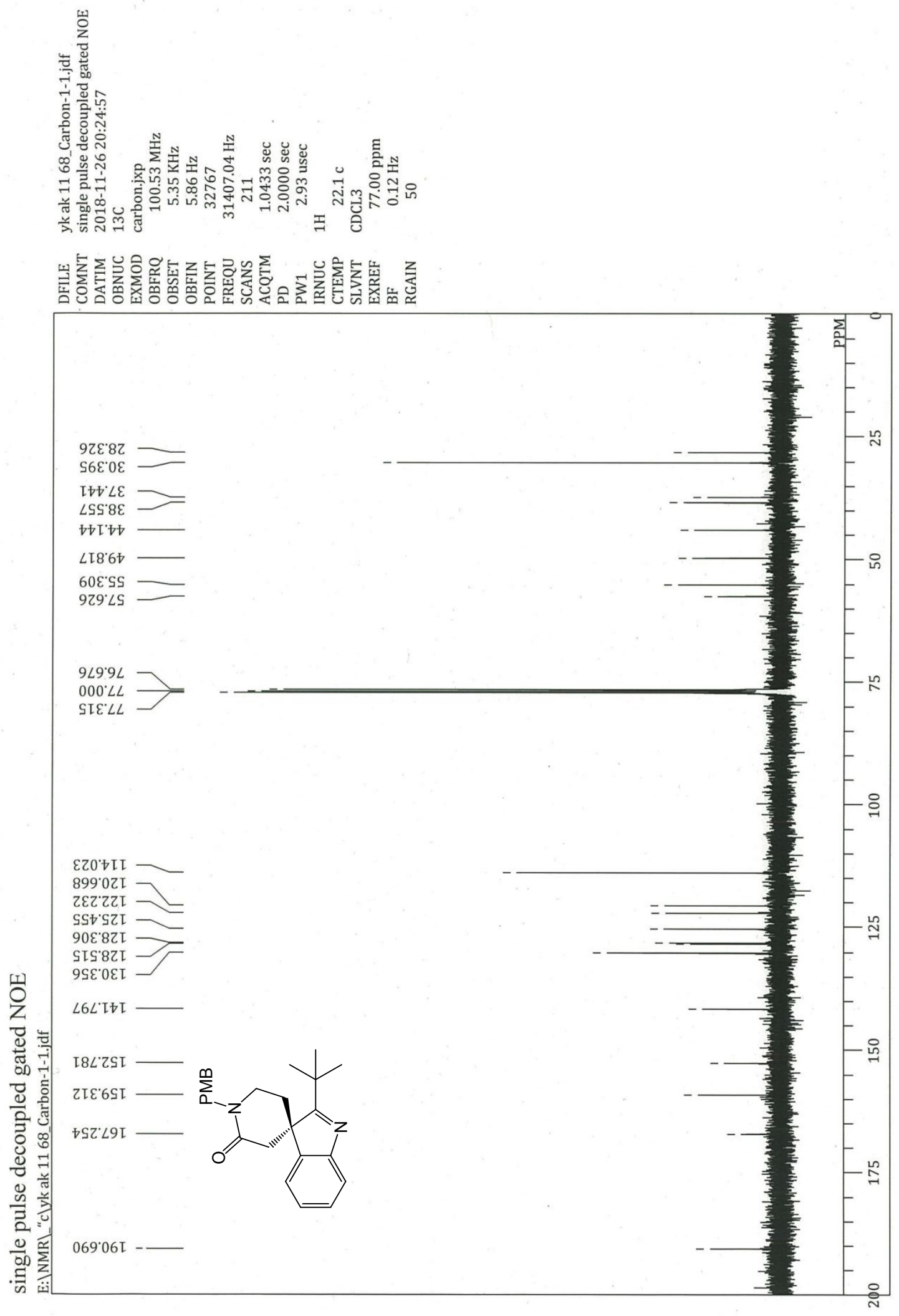


Supporting Information

$3 k$
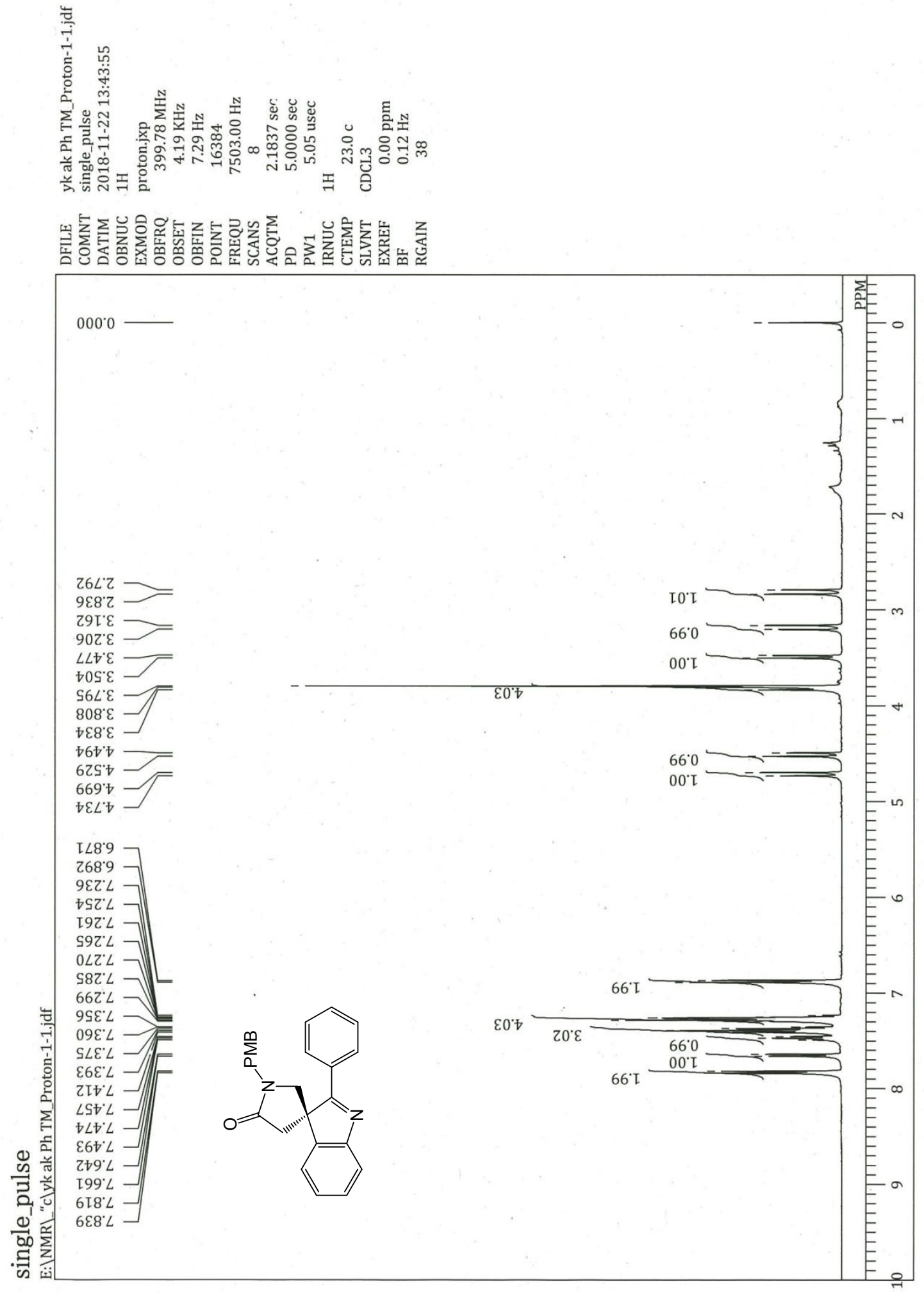
Supporting Information

$3 k$

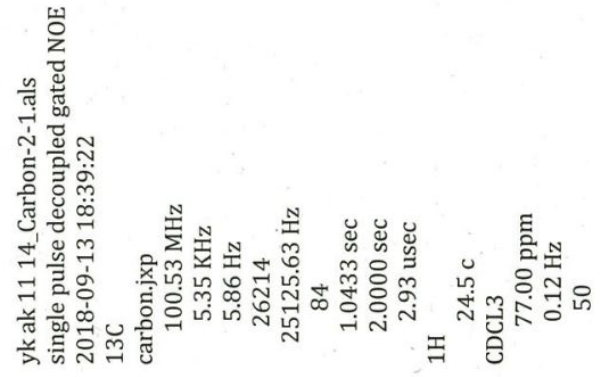

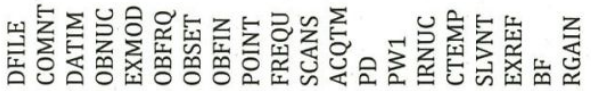

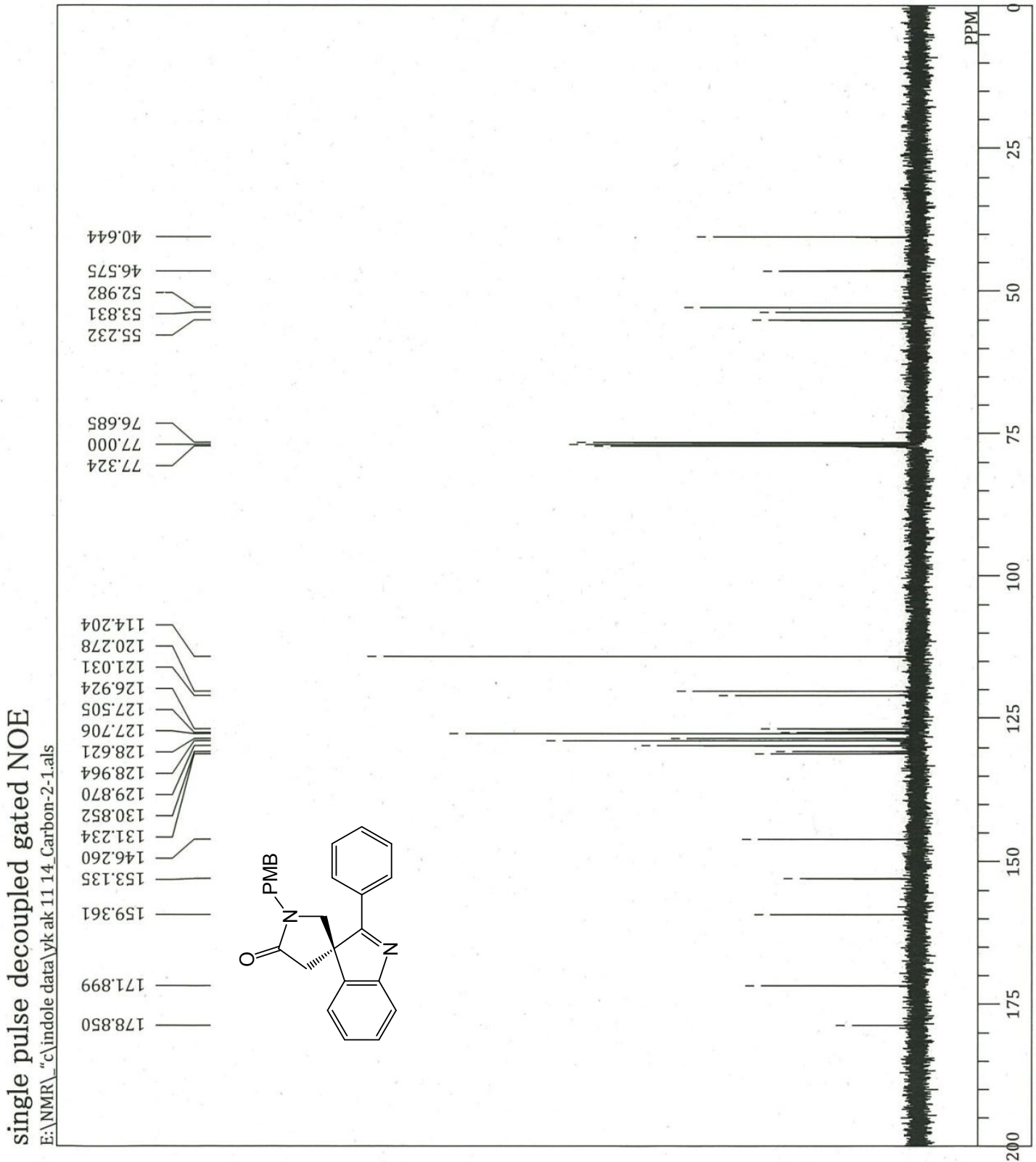


Supporting Information

31

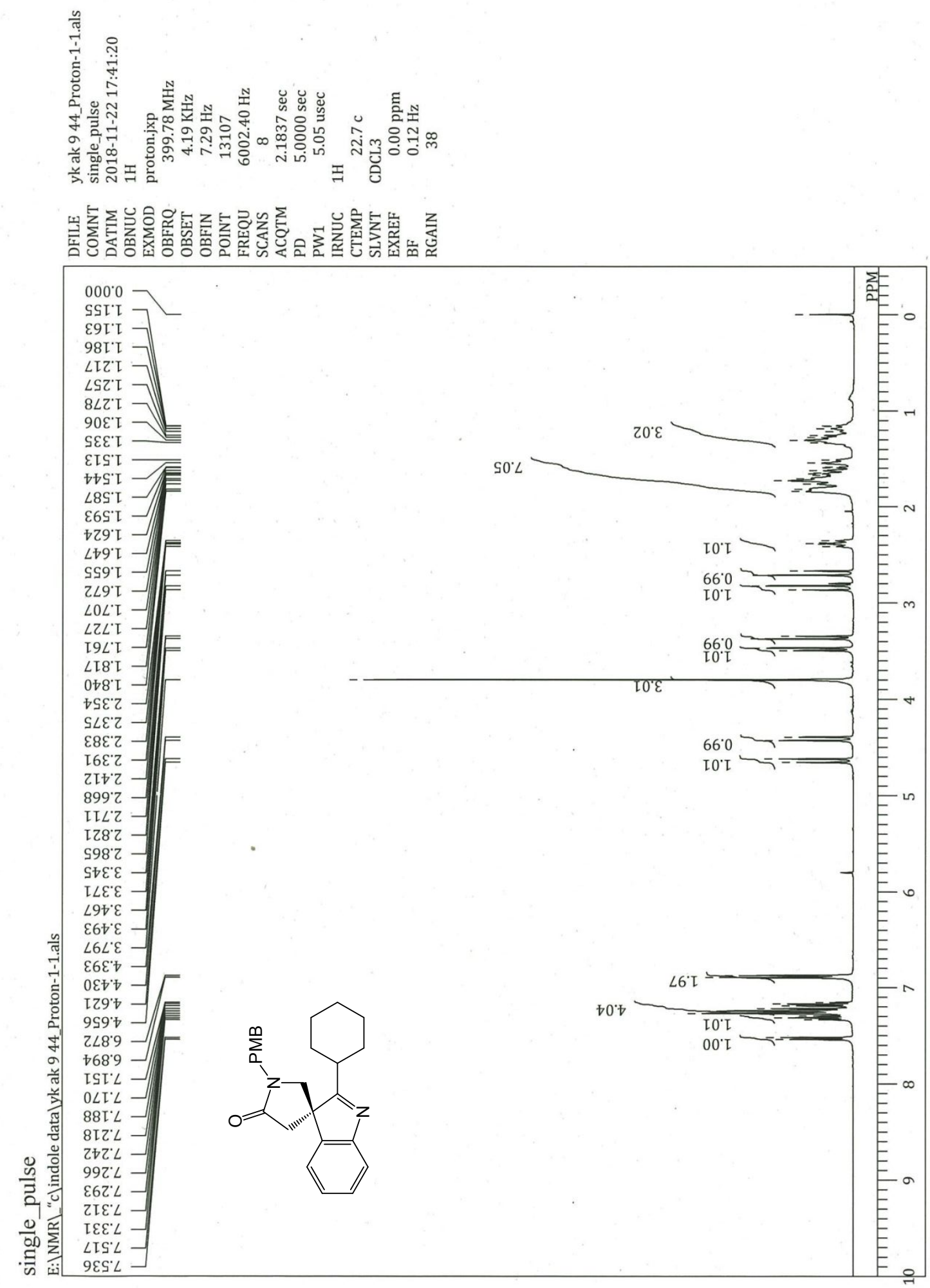


Supporting Information

31

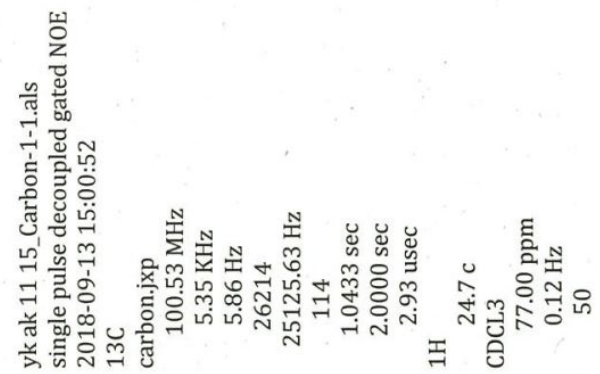

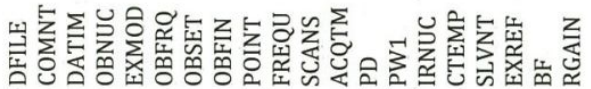

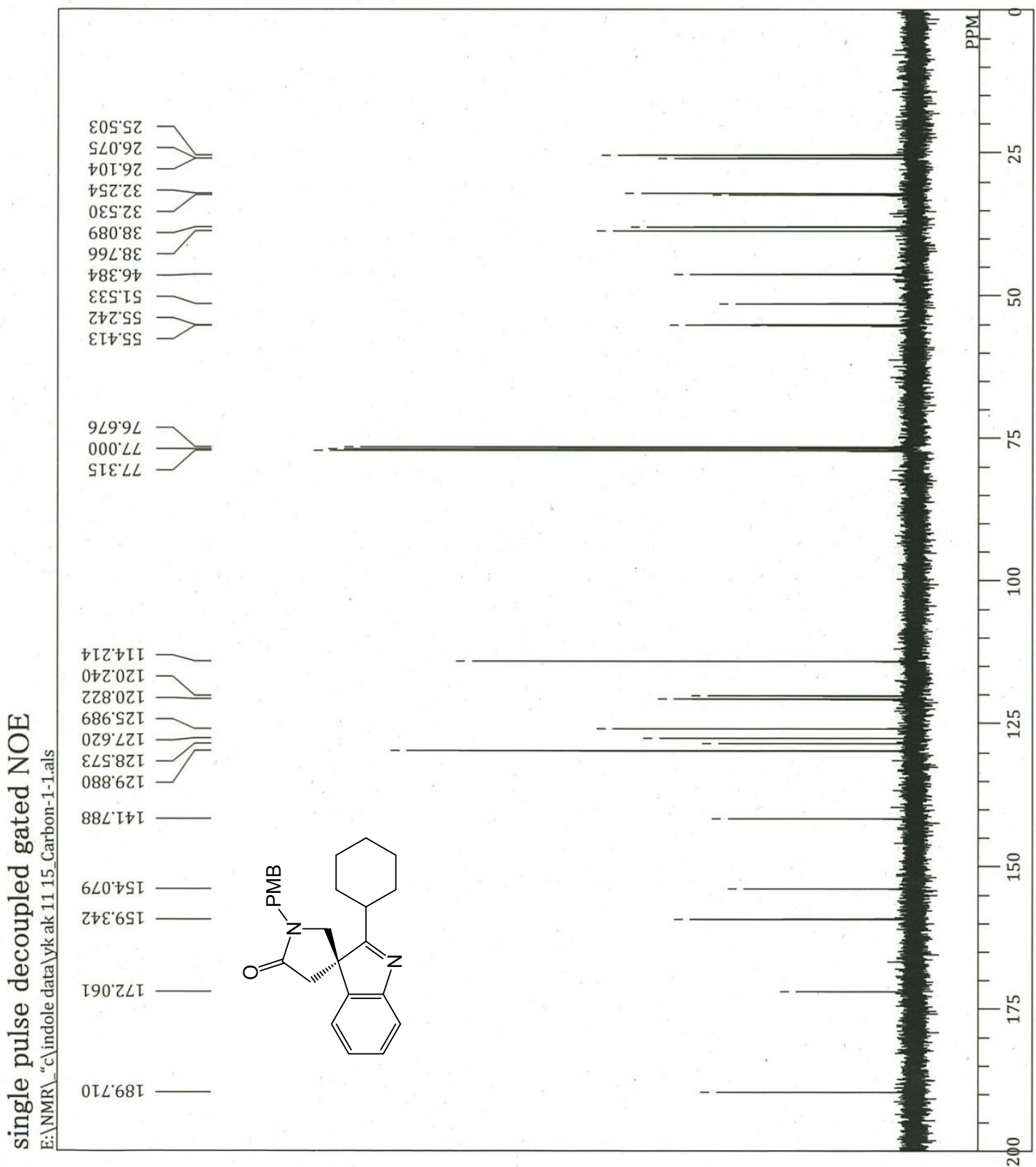


Supporting Information

$3 \mathrm{~m}$
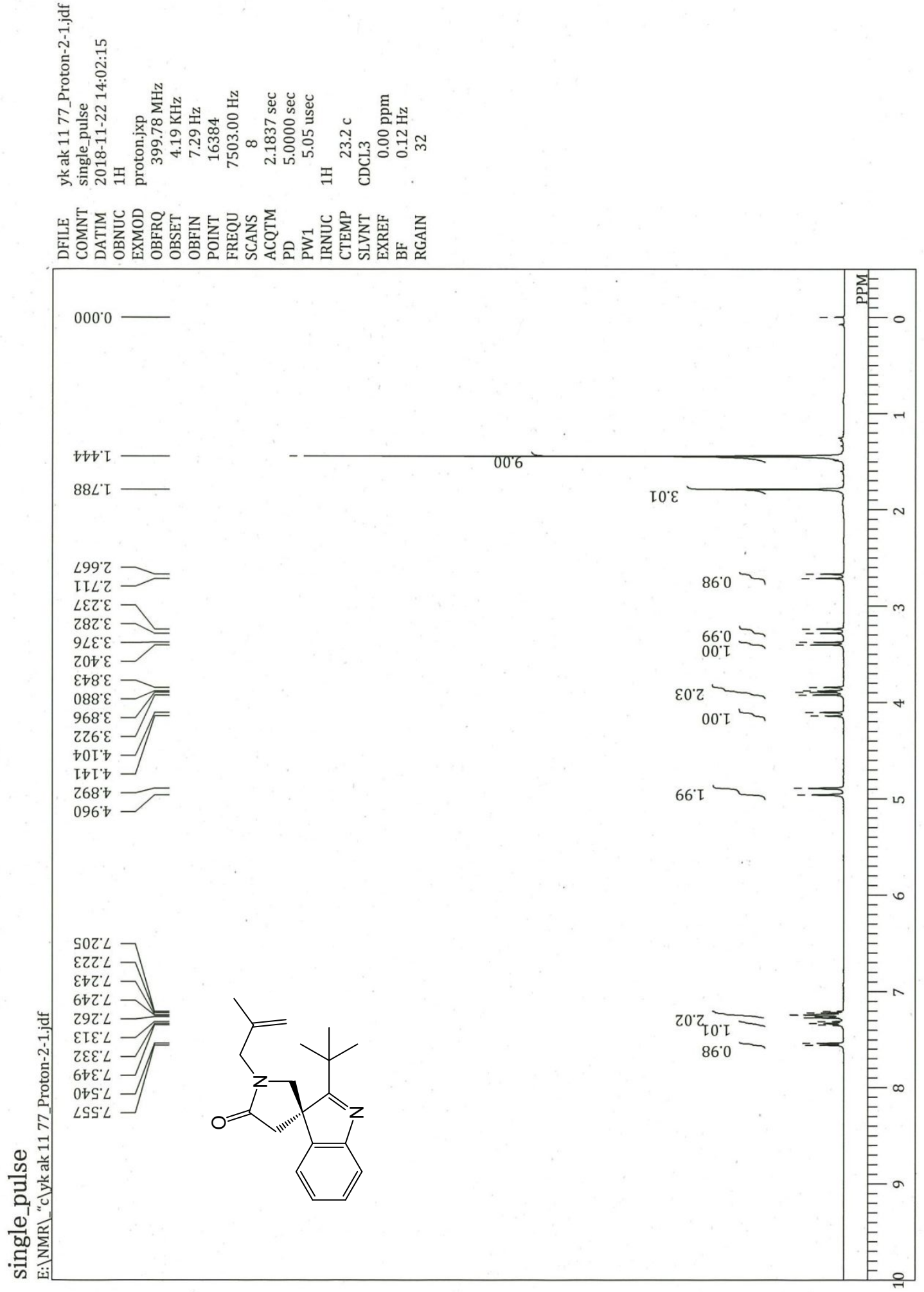
Supporting Information

$3 \mathrm{~m}$

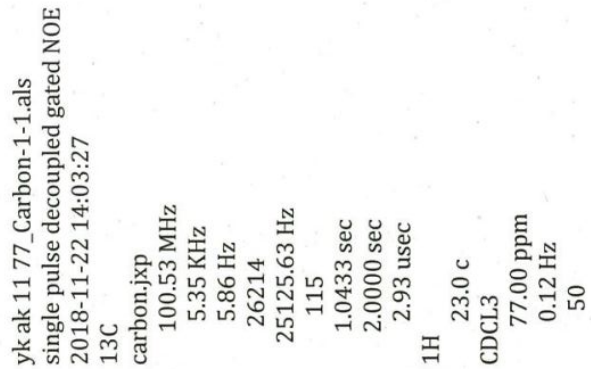

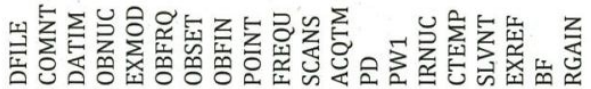

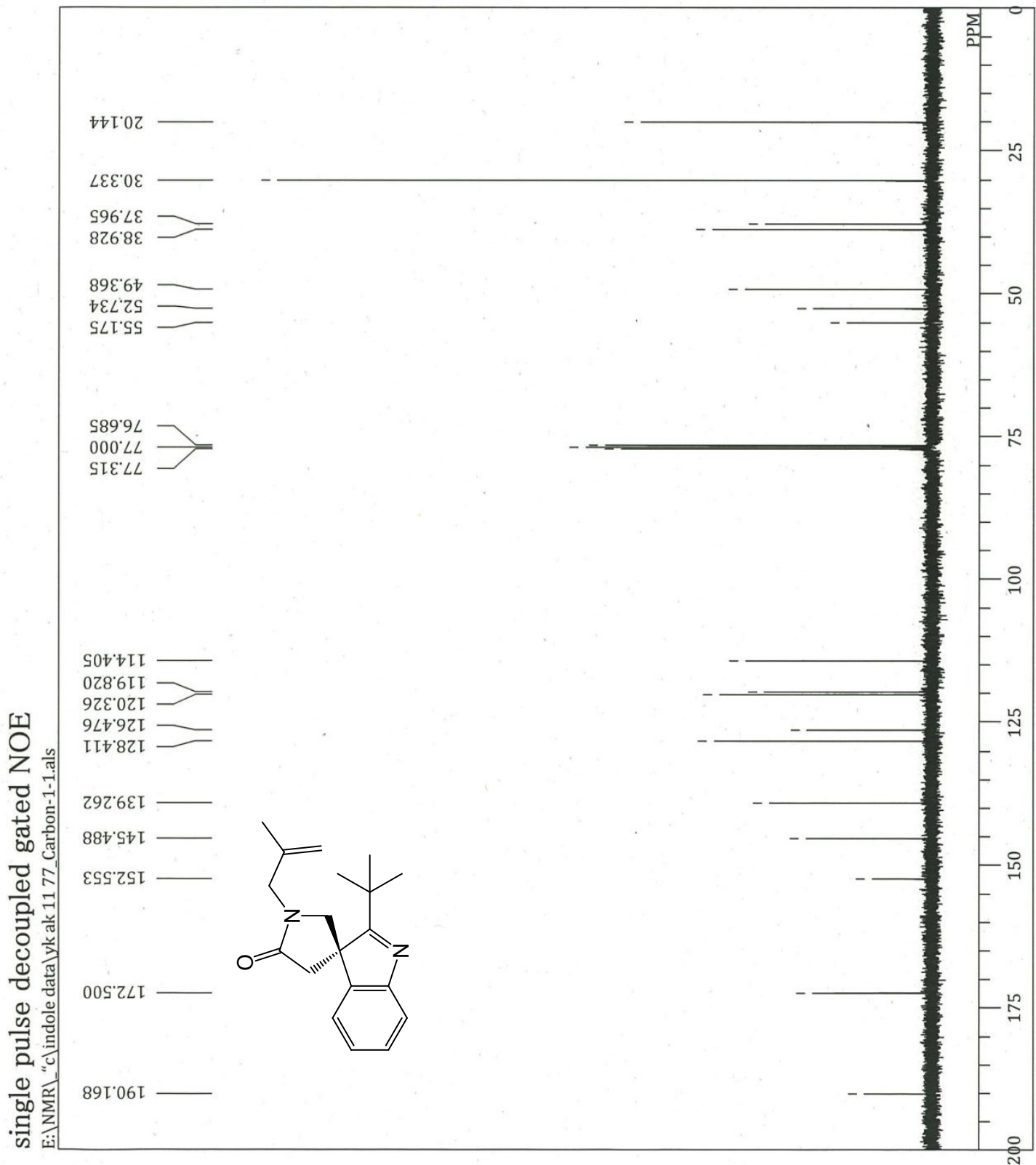


Supporting Information

$3 n$

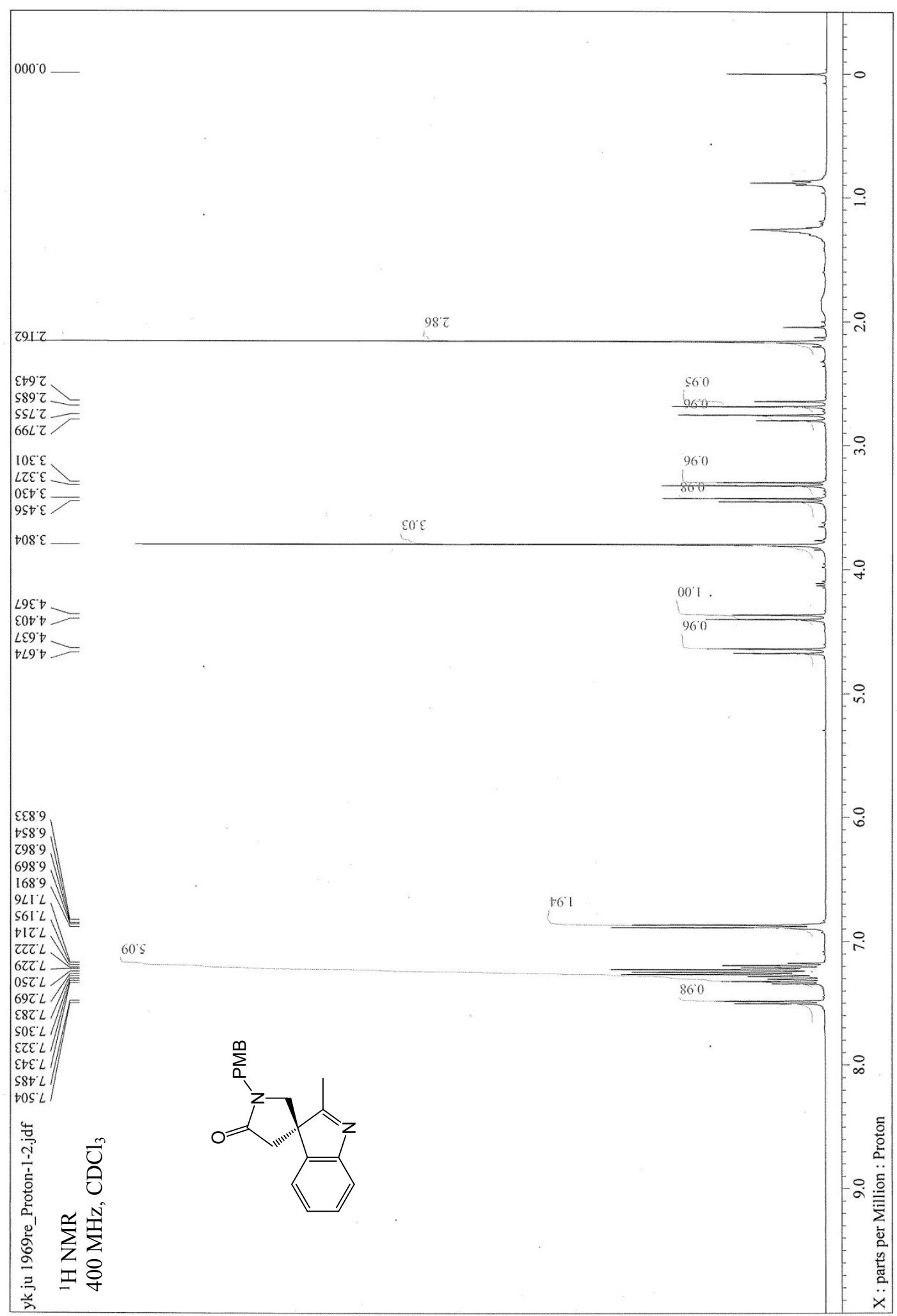


Supporting Information

$3 n$

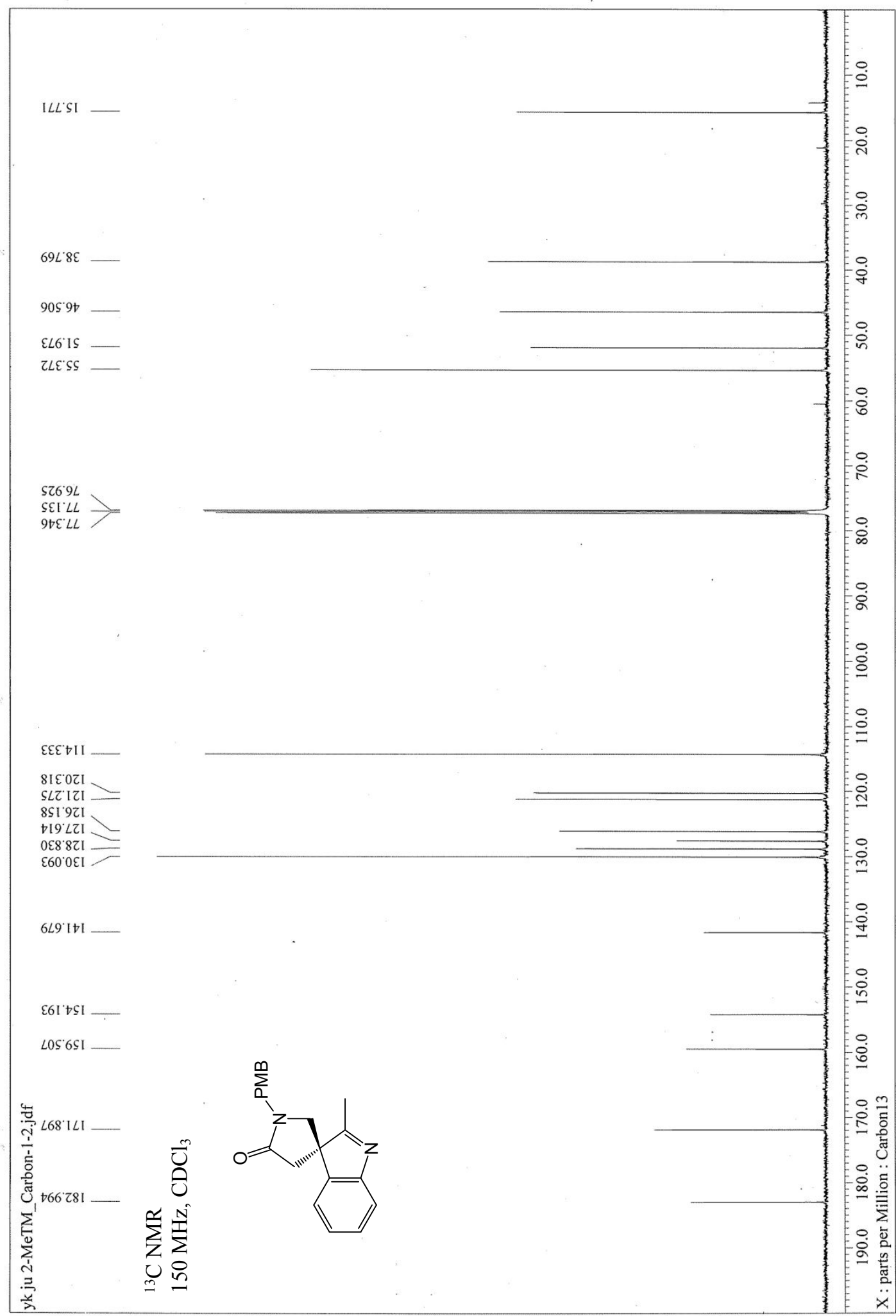


Supporting Information

S1o

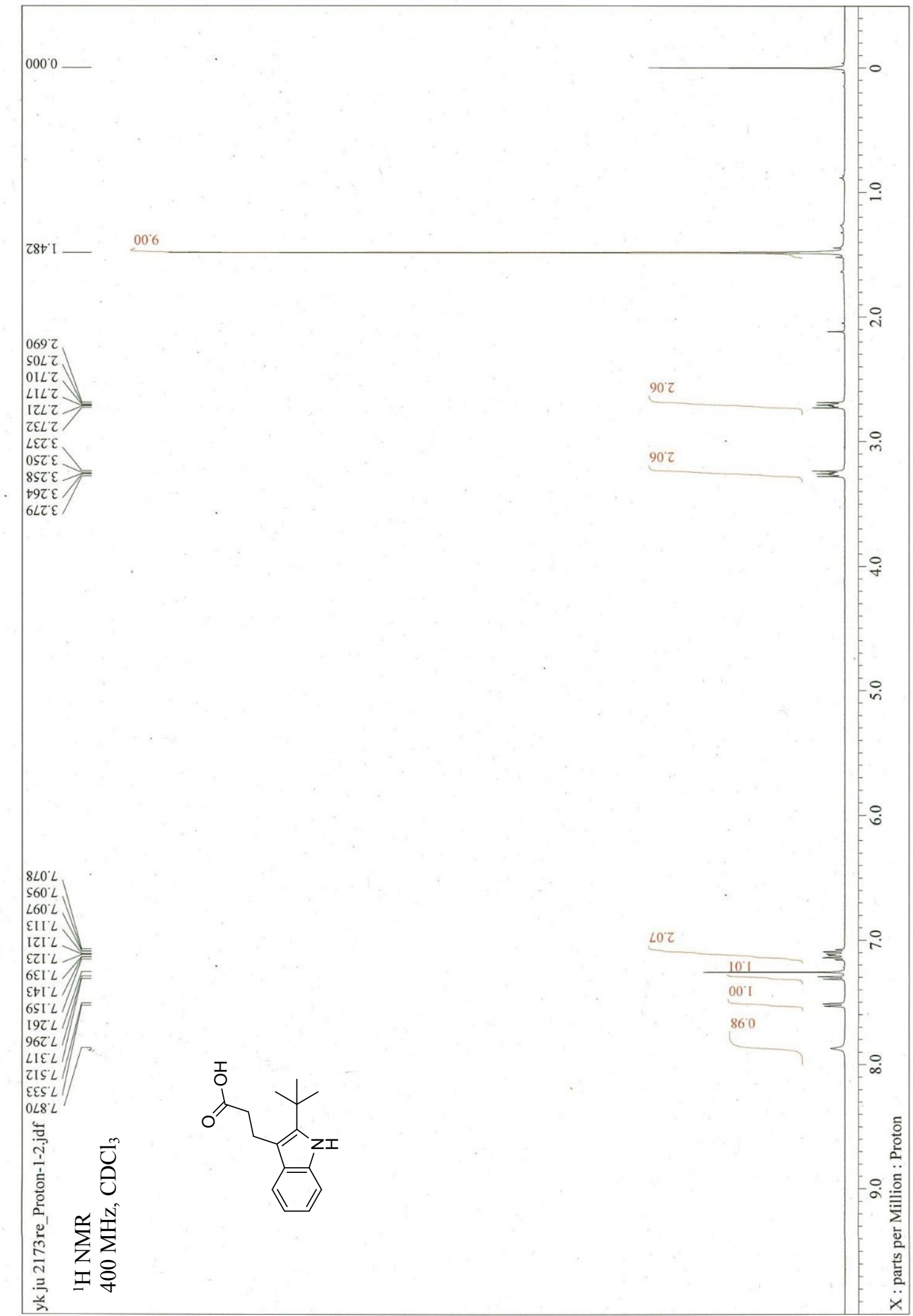


Supporting Information

S1o

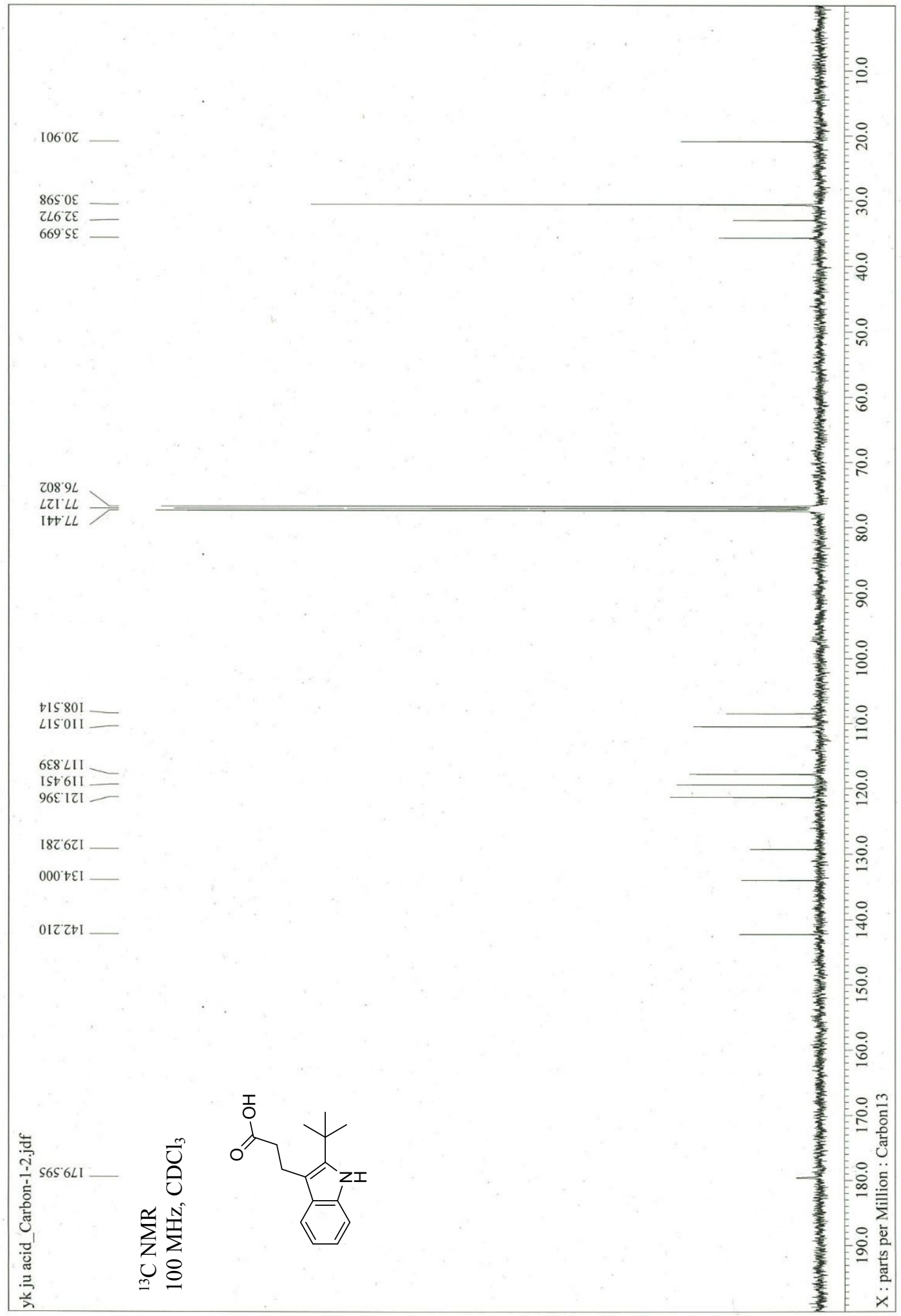


Supporting Information

$4 m$

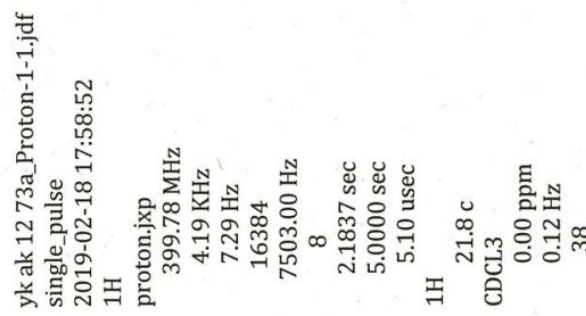

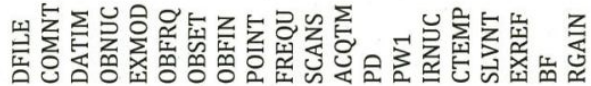

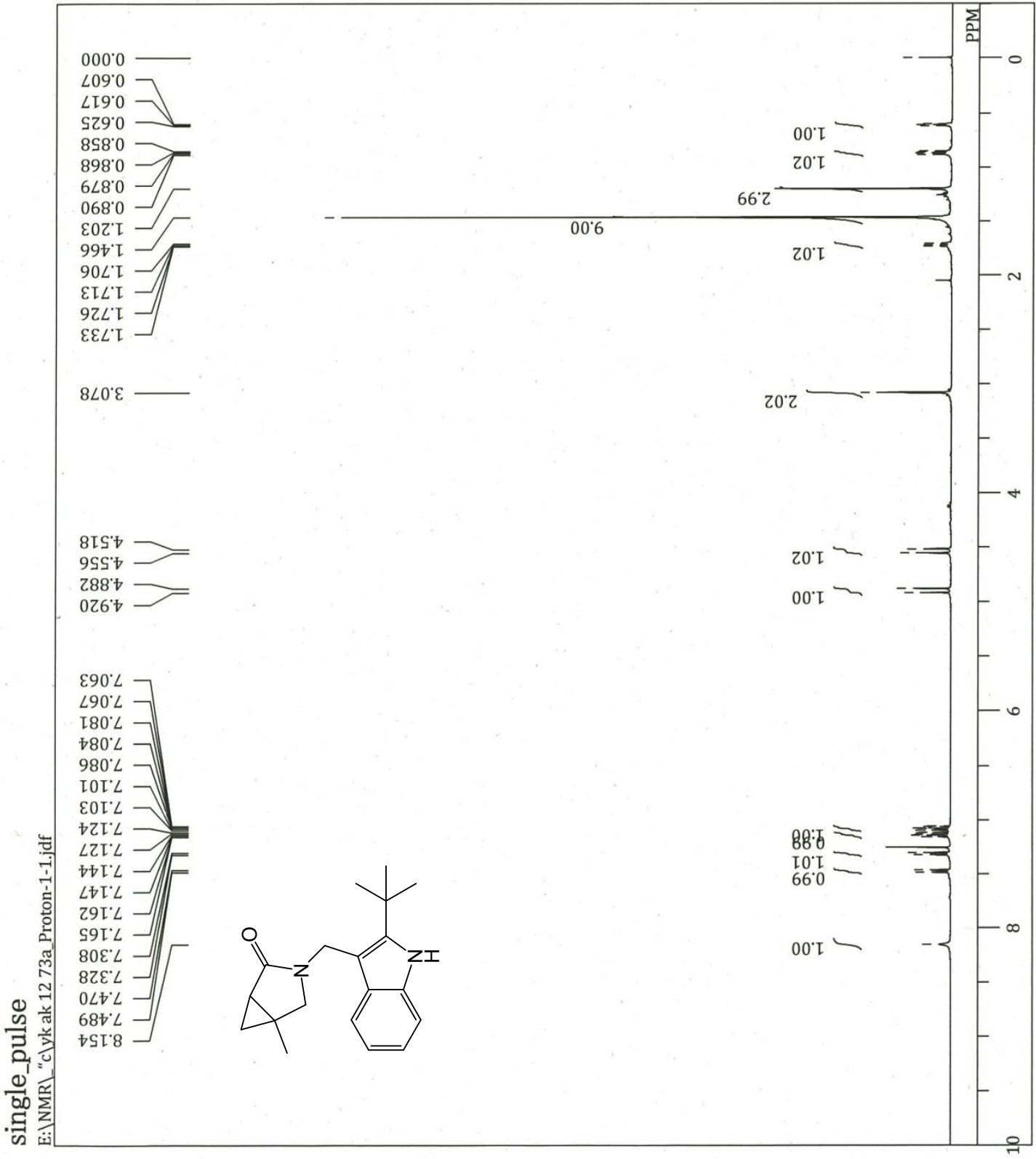


Supporting Information

$4 \mathrm{~m}$

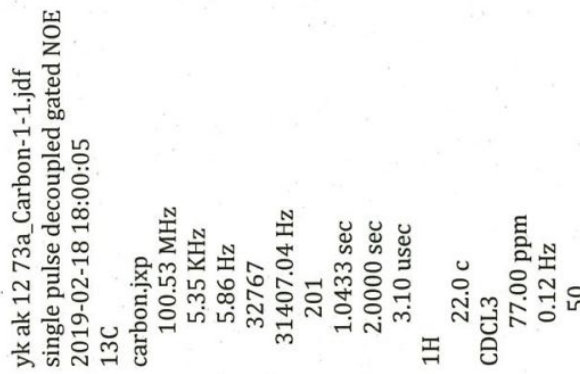

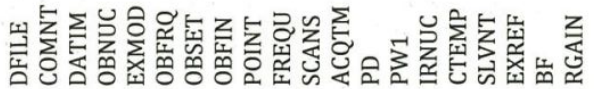

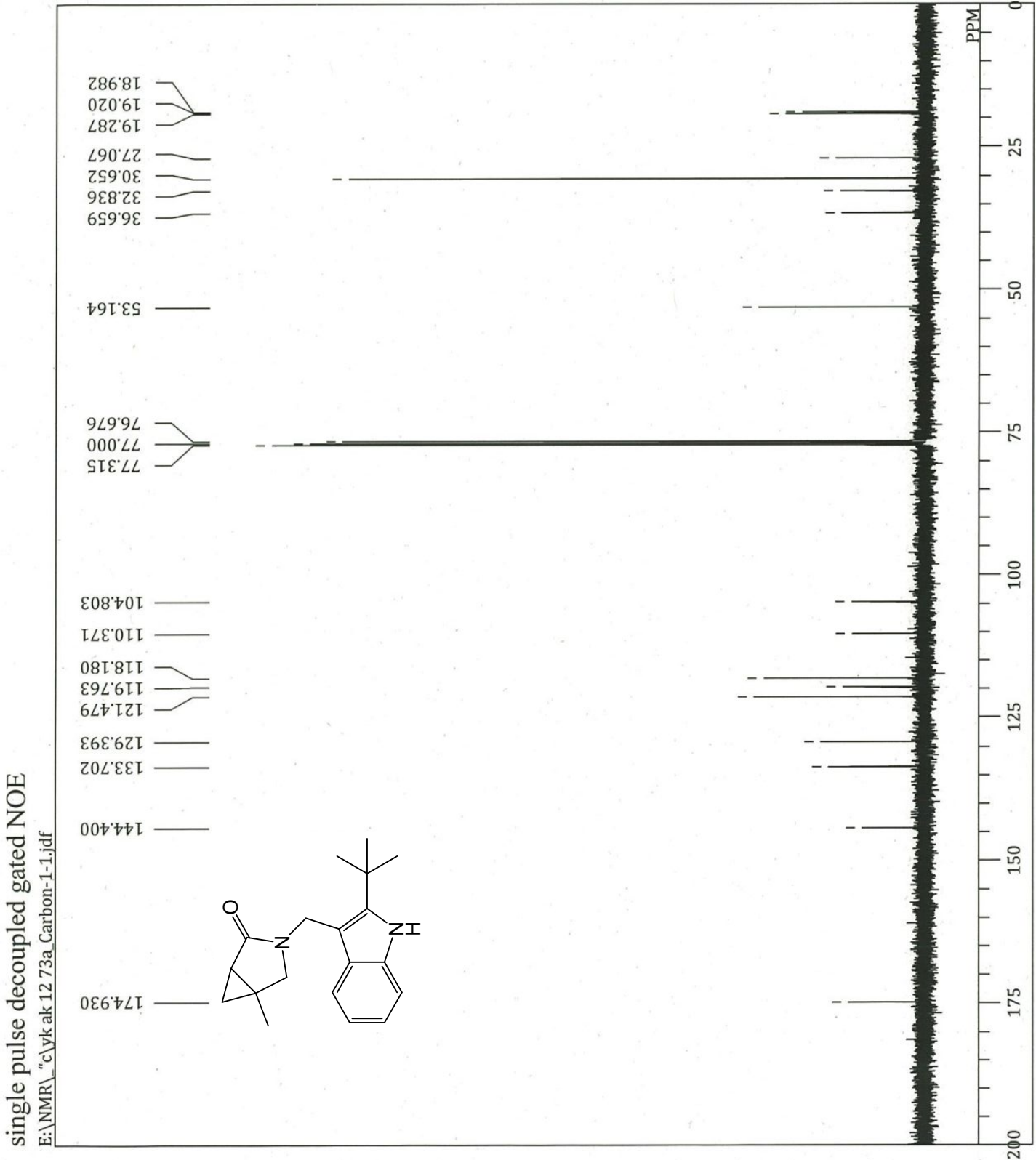


Supporting Information

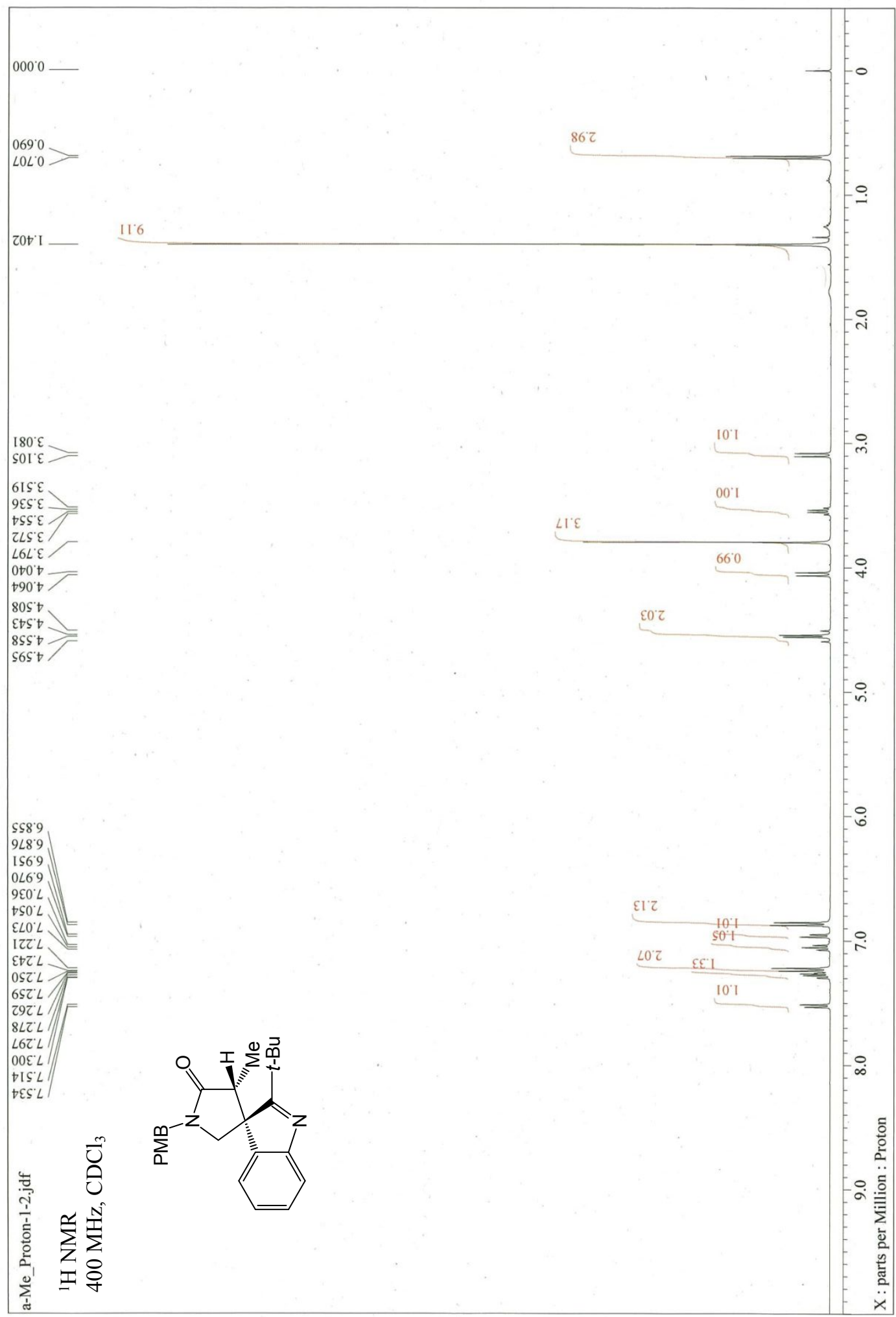


Supporting Information

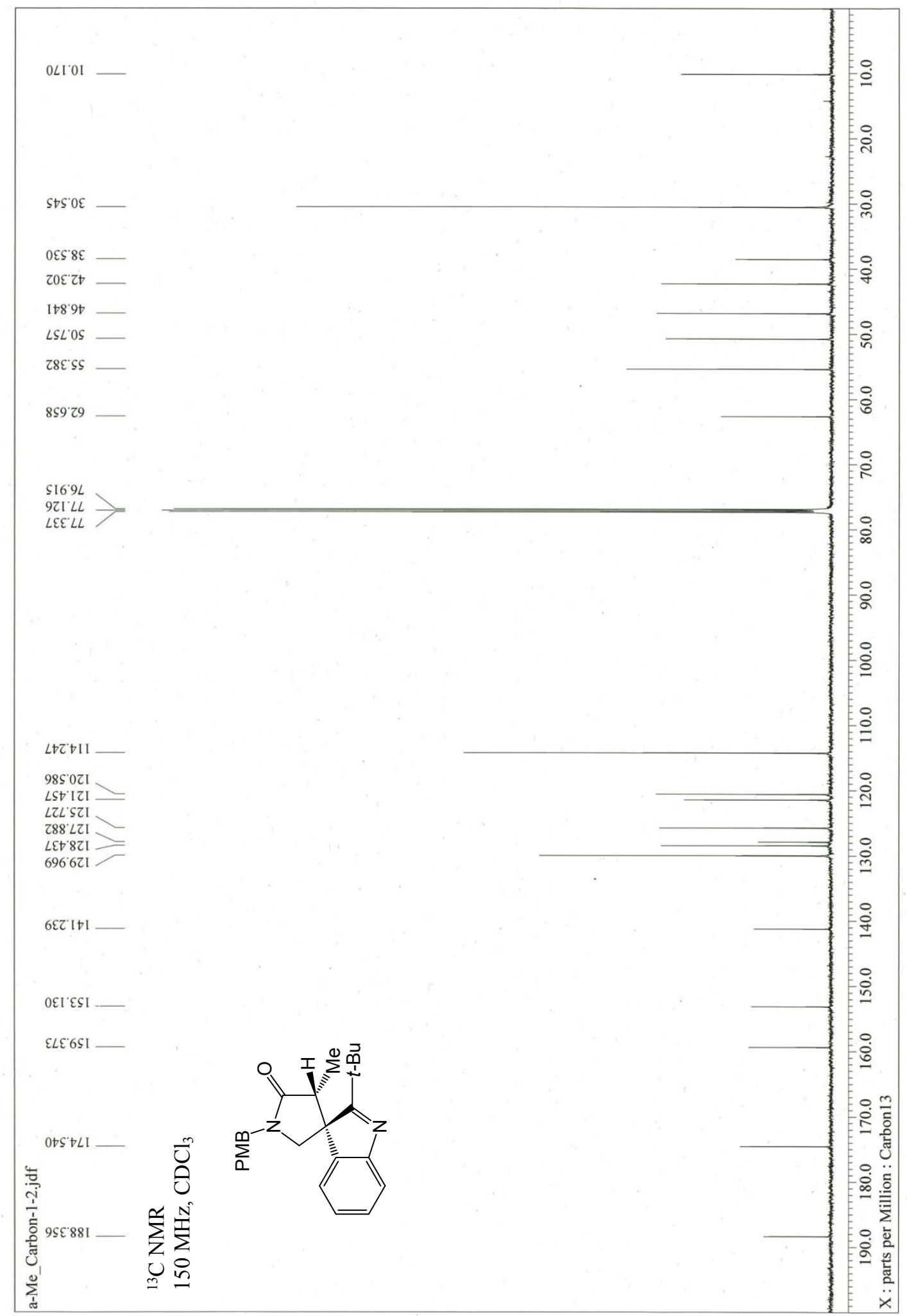


Supporting Information

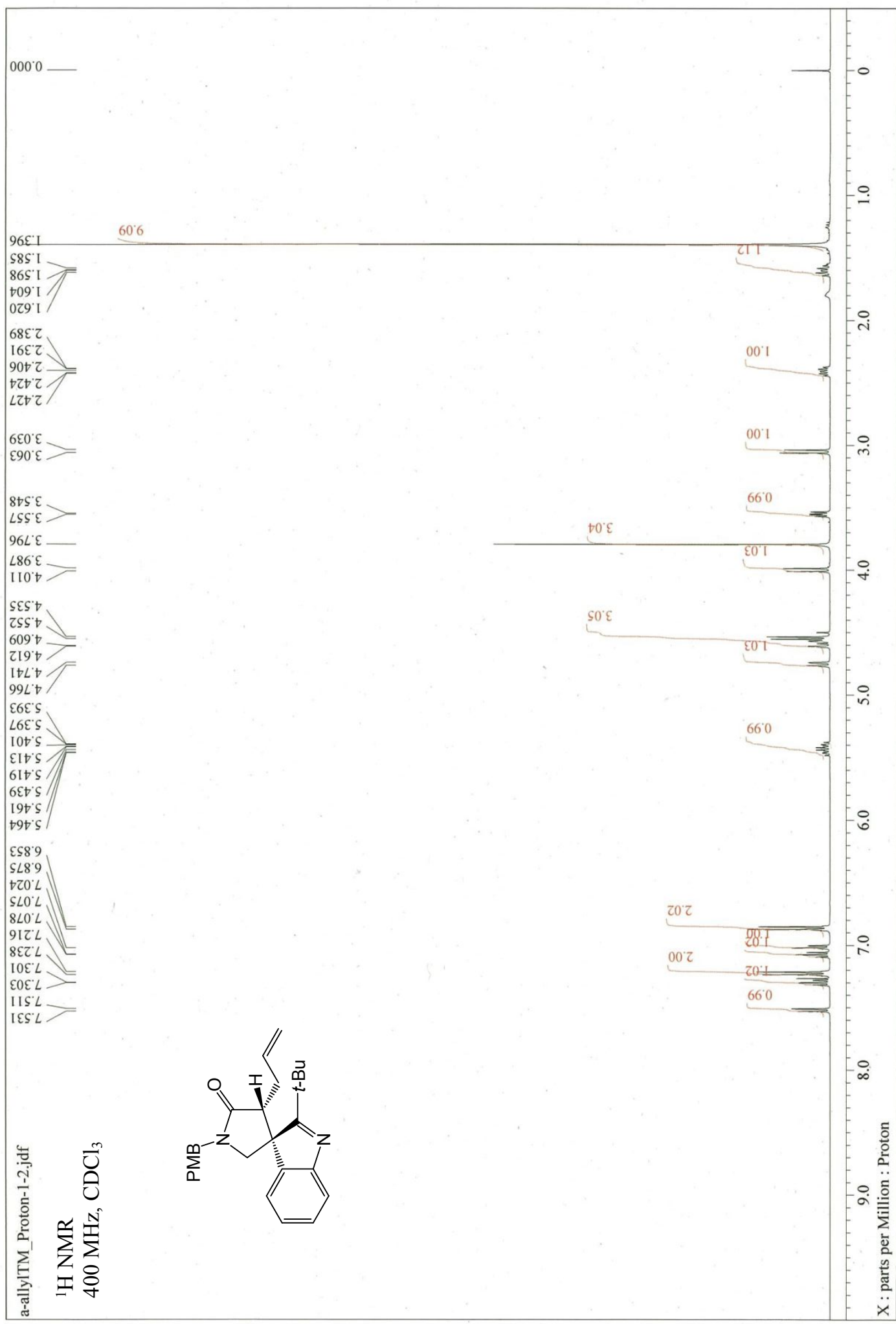


Supporting Information

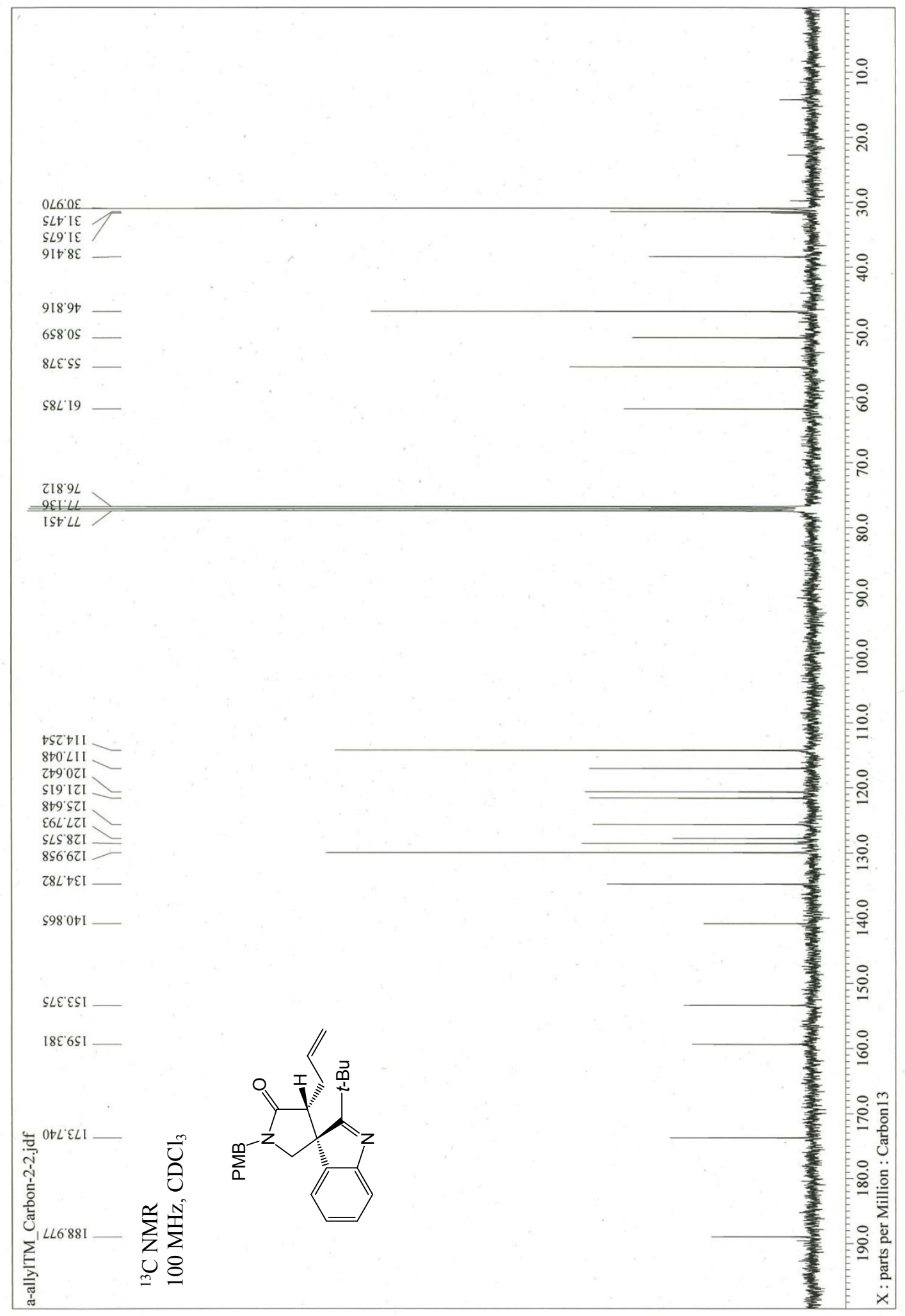


Supporting Information

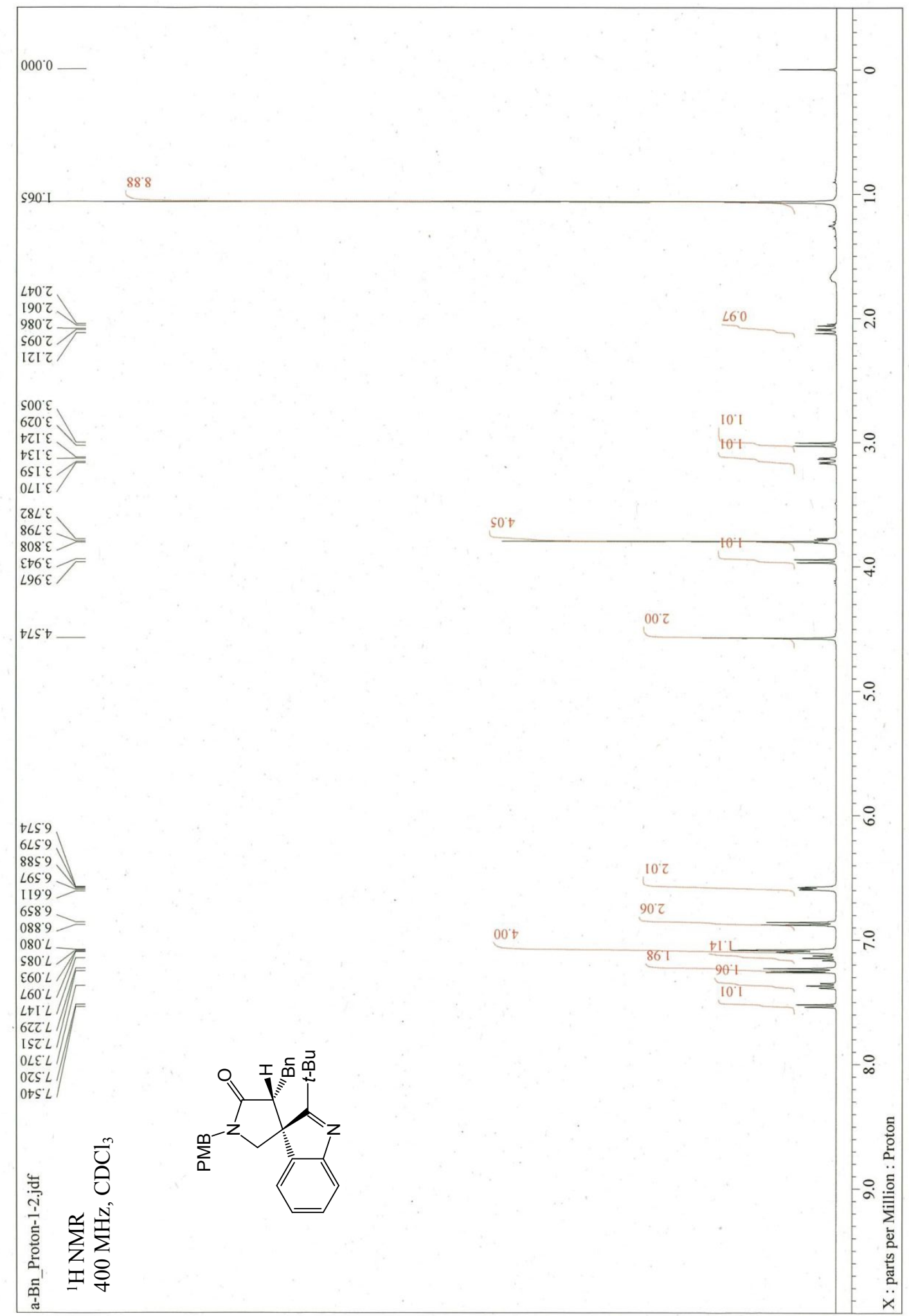


Supporting Information

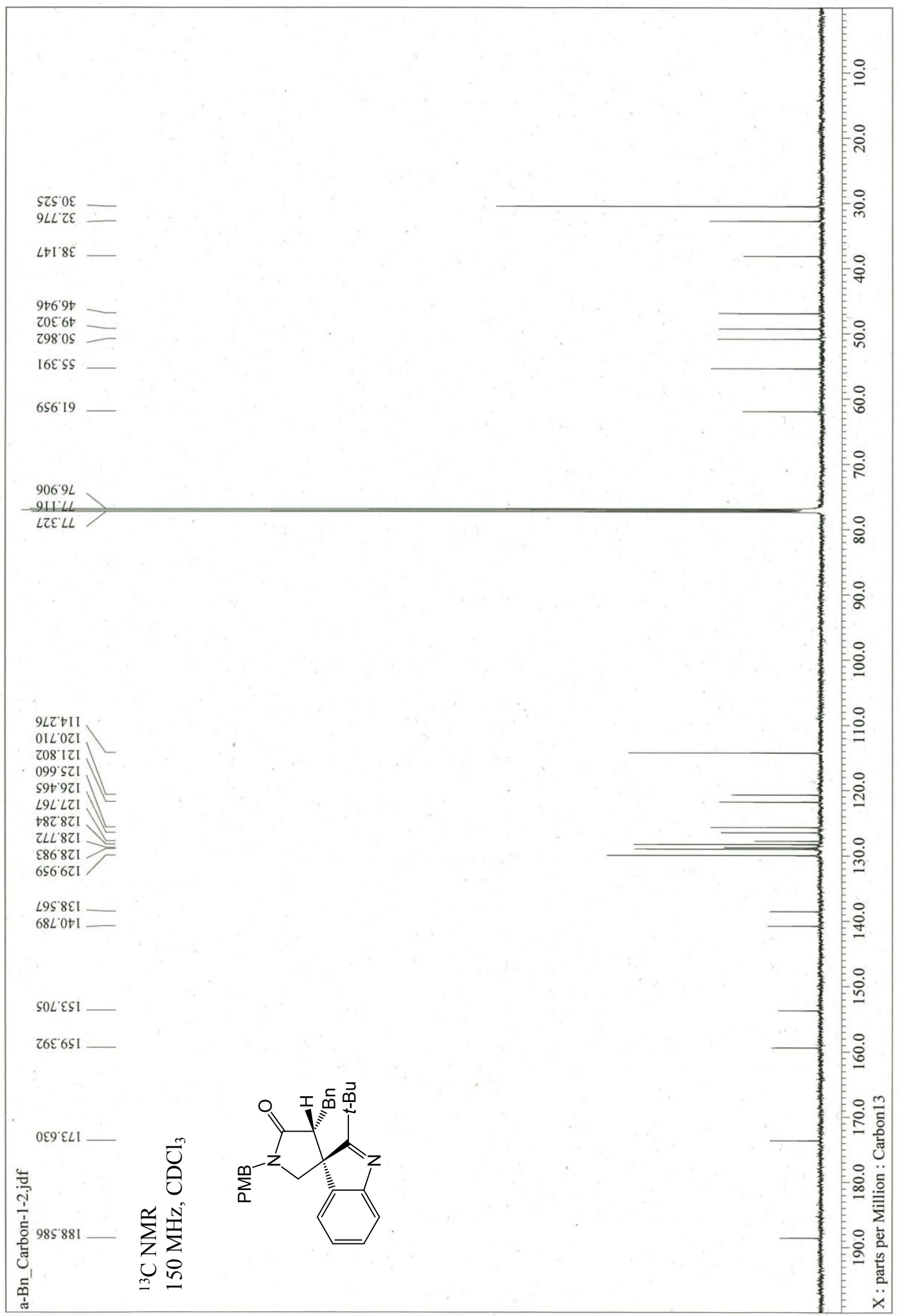


Supporting Information

8

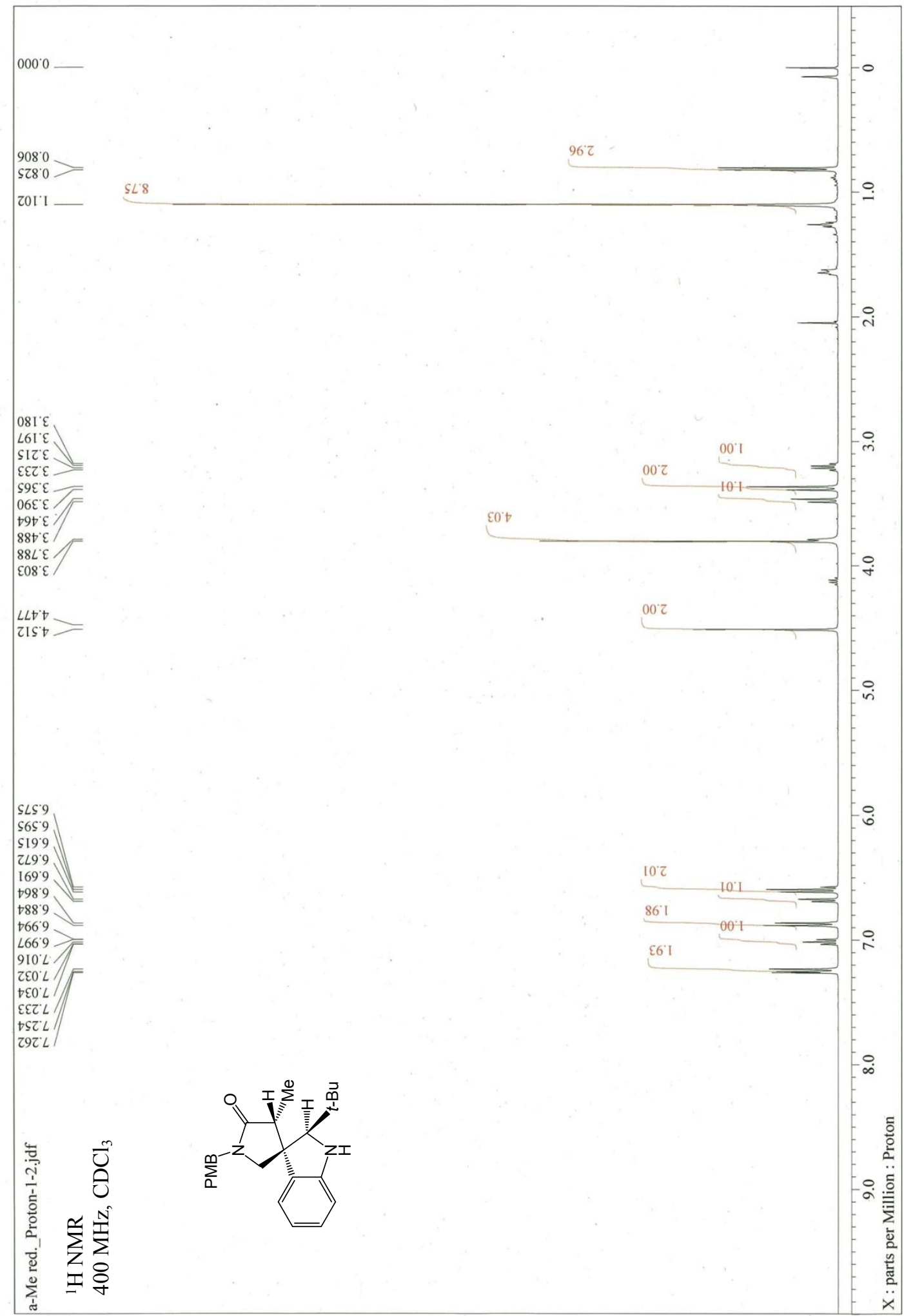


Supporting Information

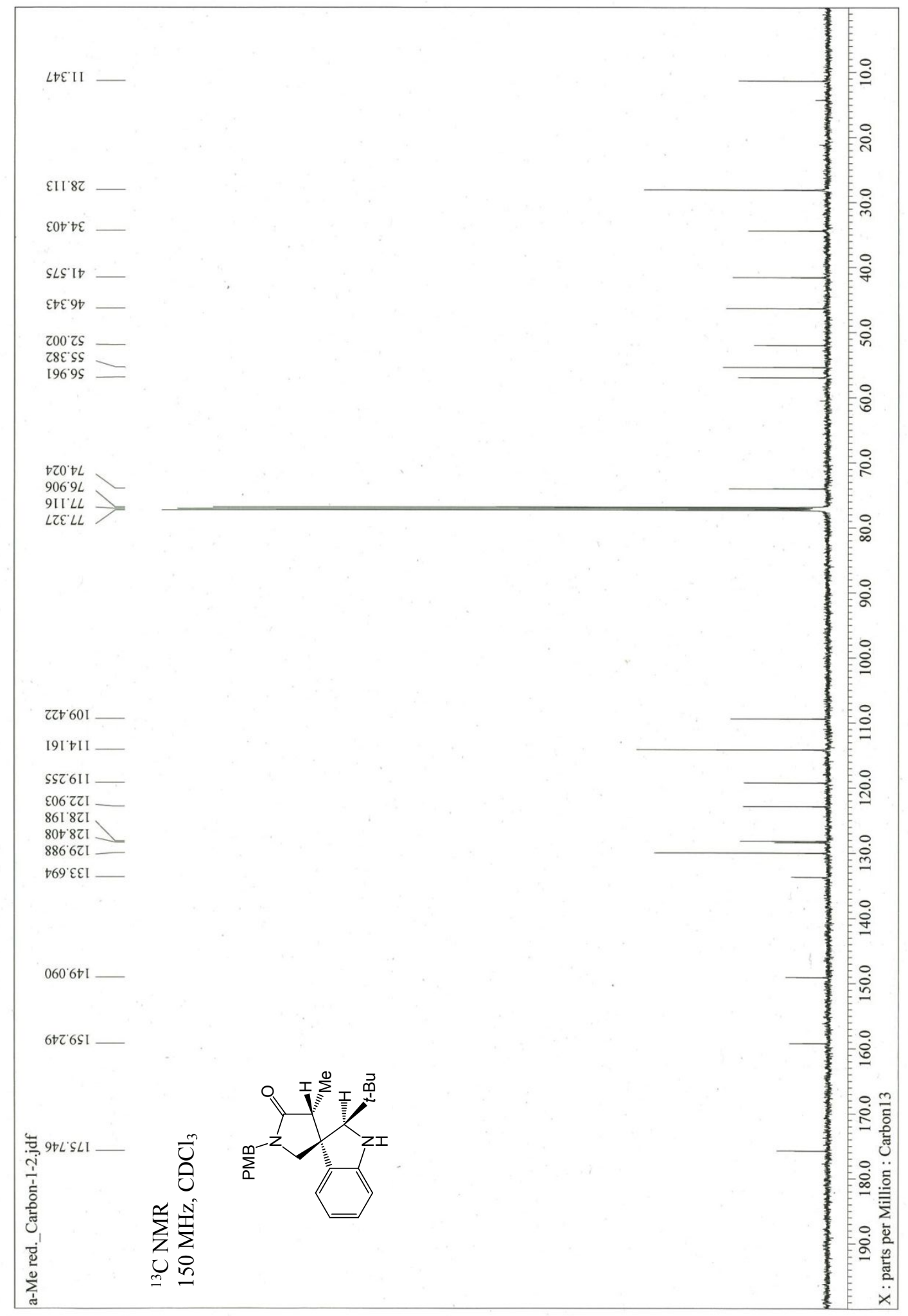


Supporting Information

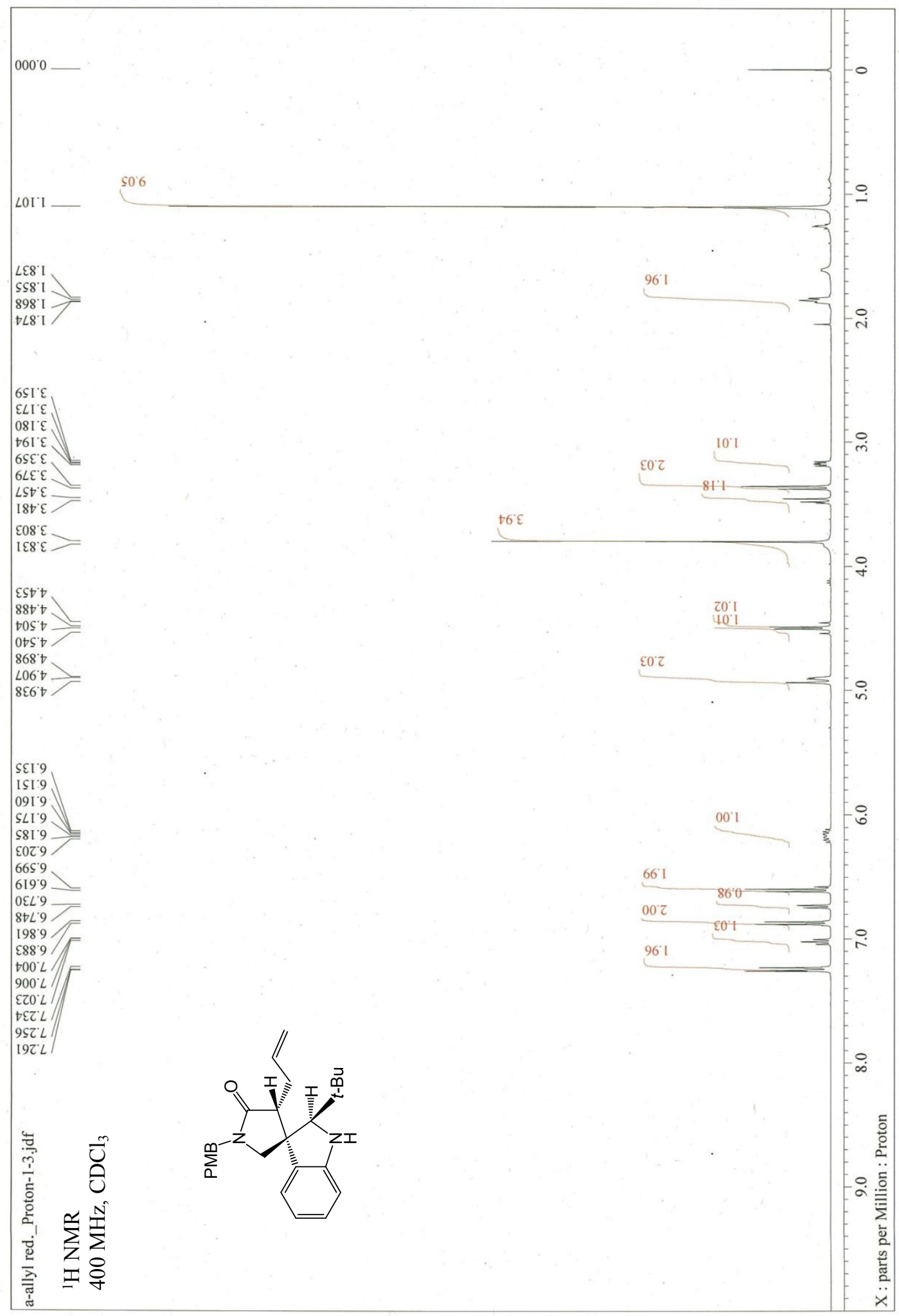


Supporting Information

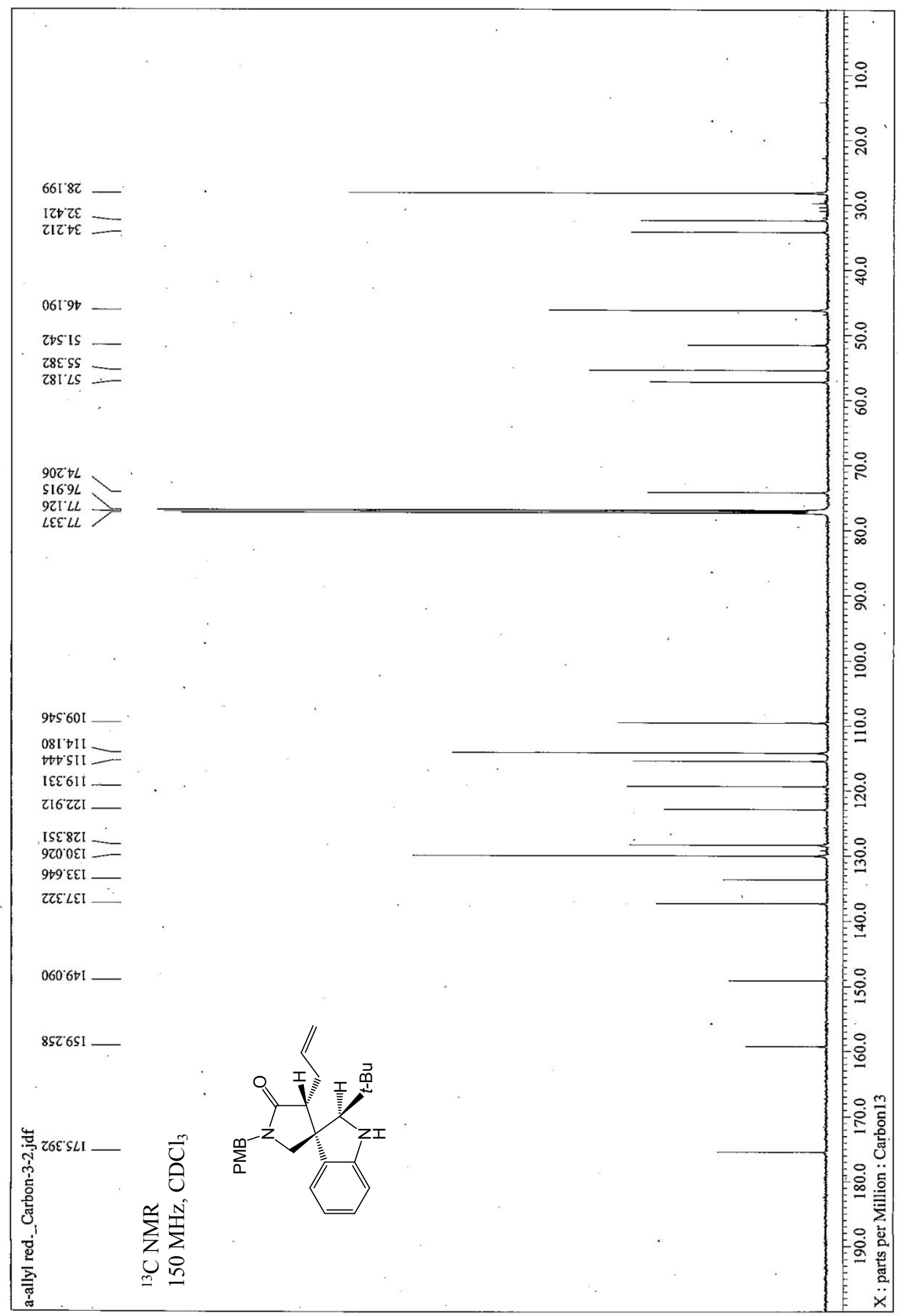


Supporting Information

10

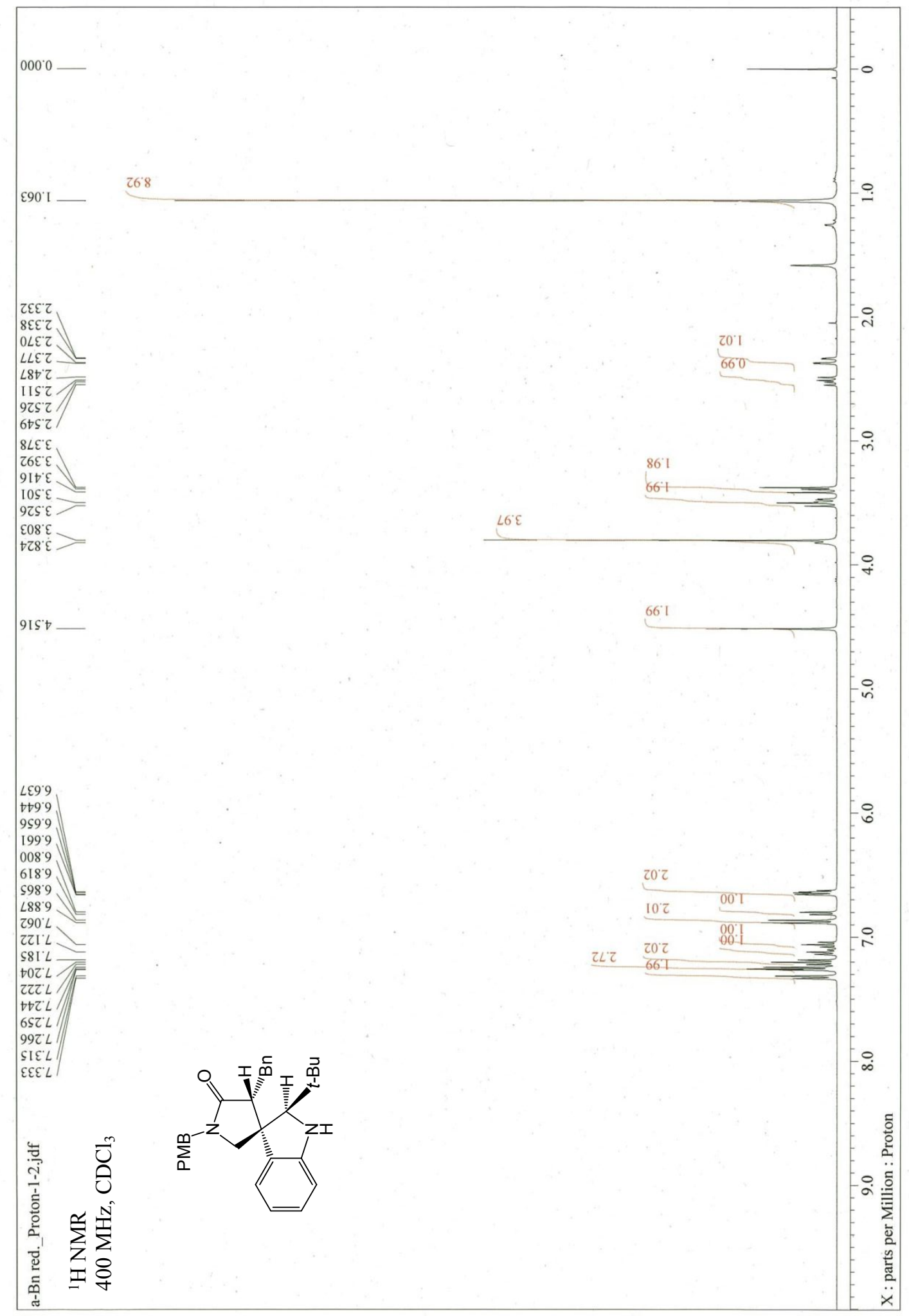


Supporting Information

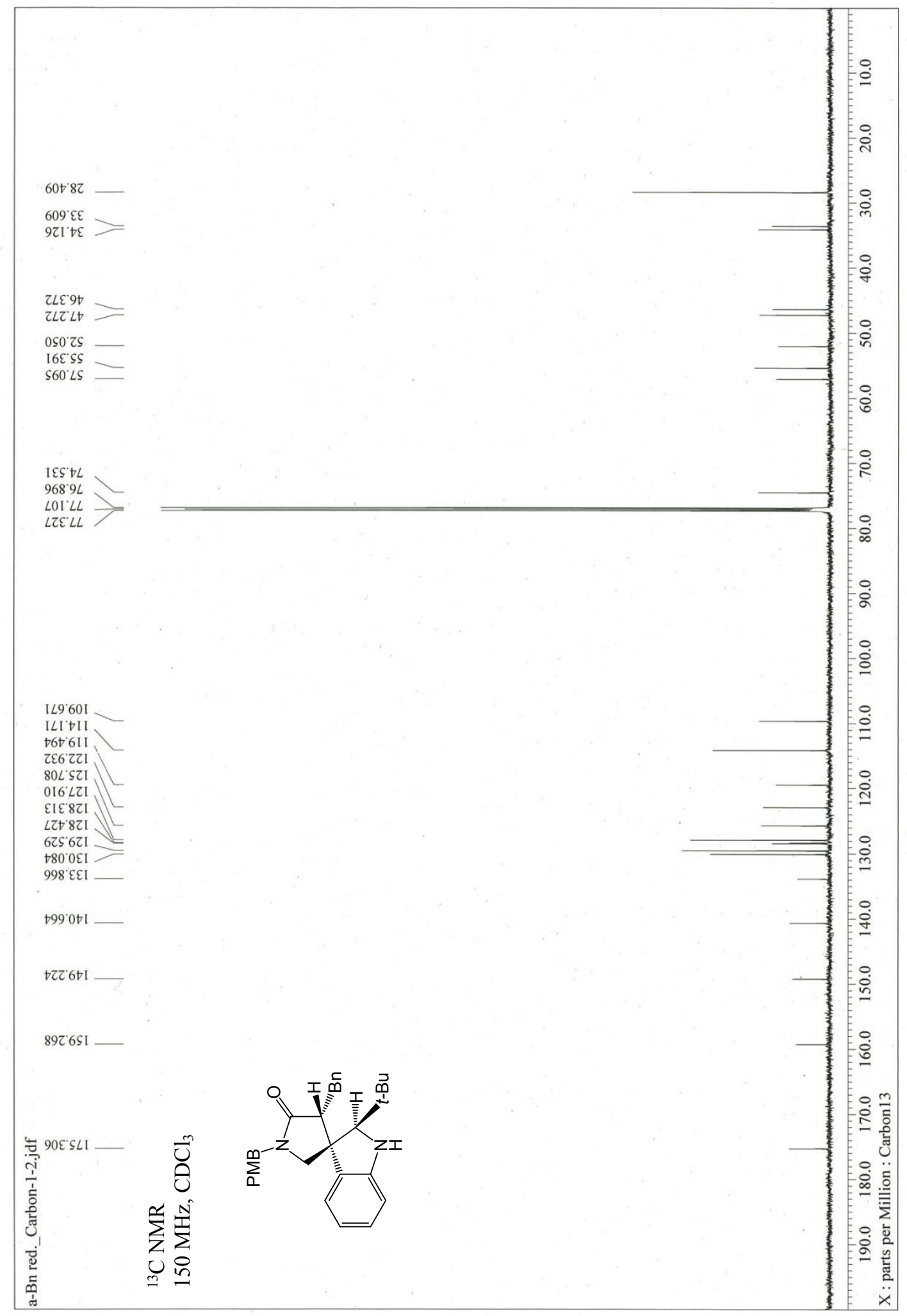


Supporting Information

11

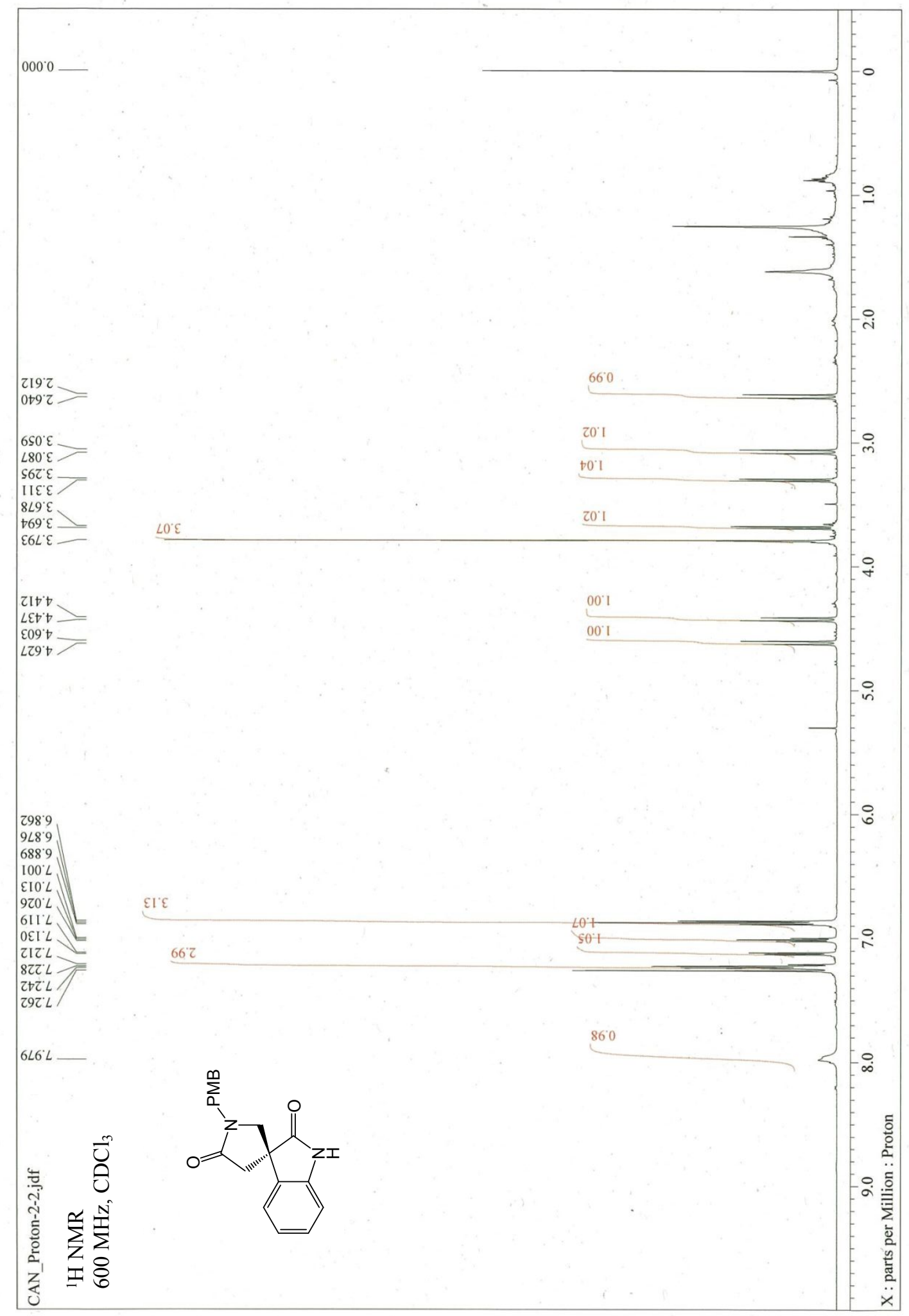


Supporting Information

11

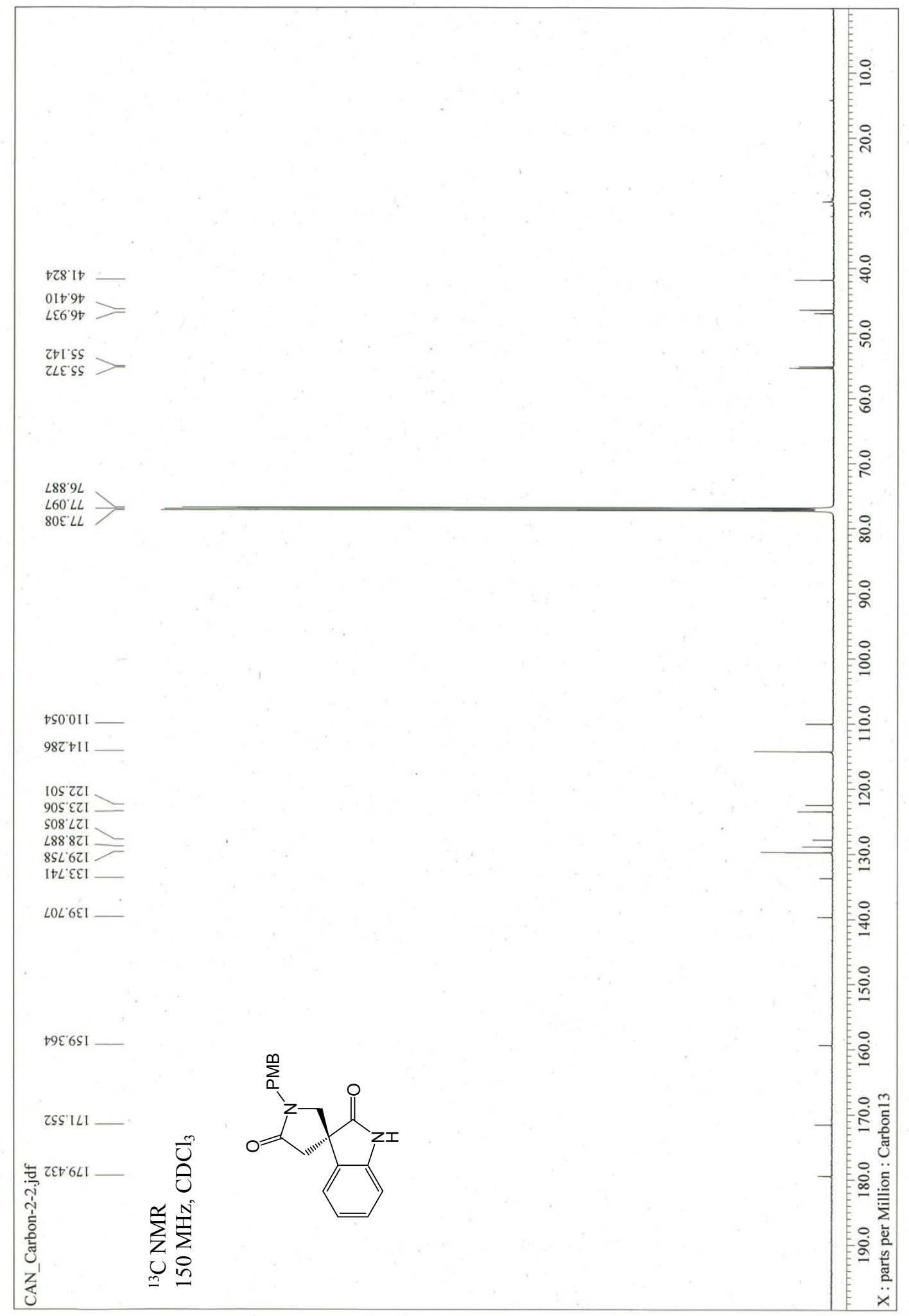


Supporting Information

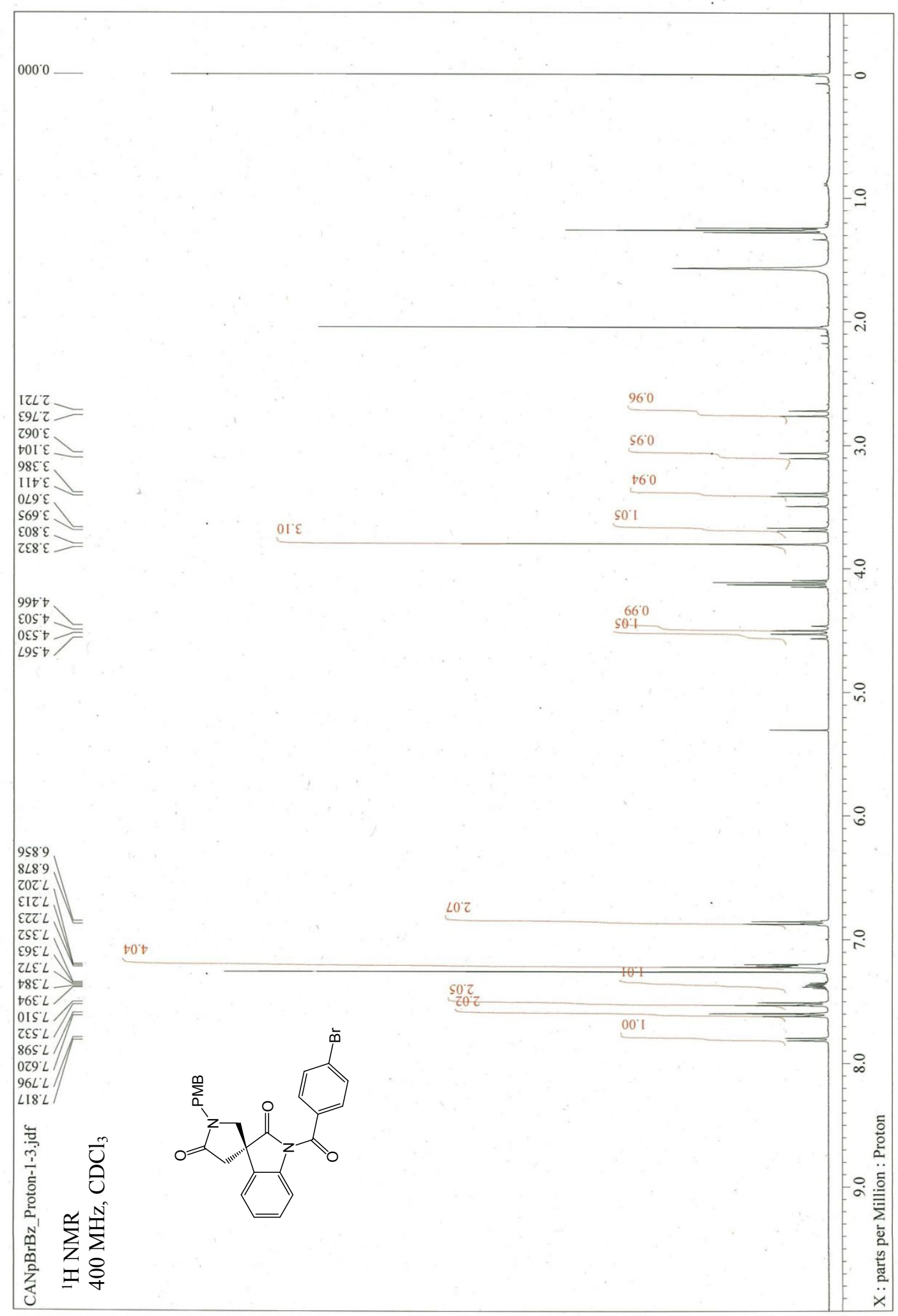


Supporting Information

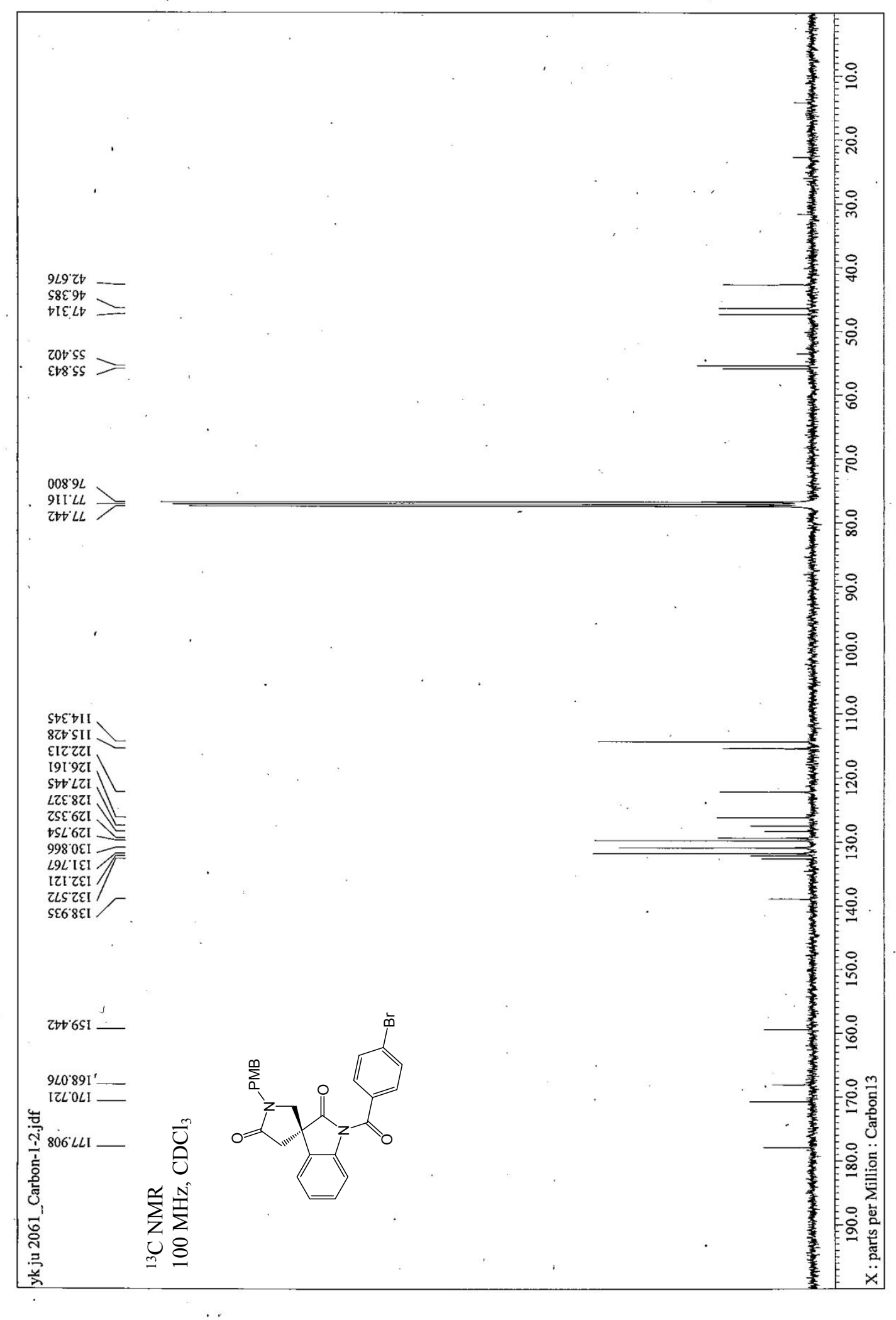


Supporting Information

\section{Chiral and Racemic HPLC Traces}

$3 a$

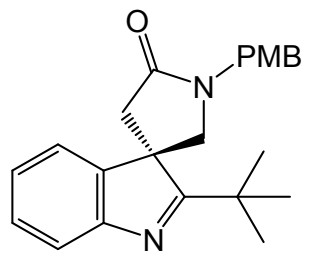

ak 8-32_0123 ak 8-32_004 2018/12/18 21:30:33

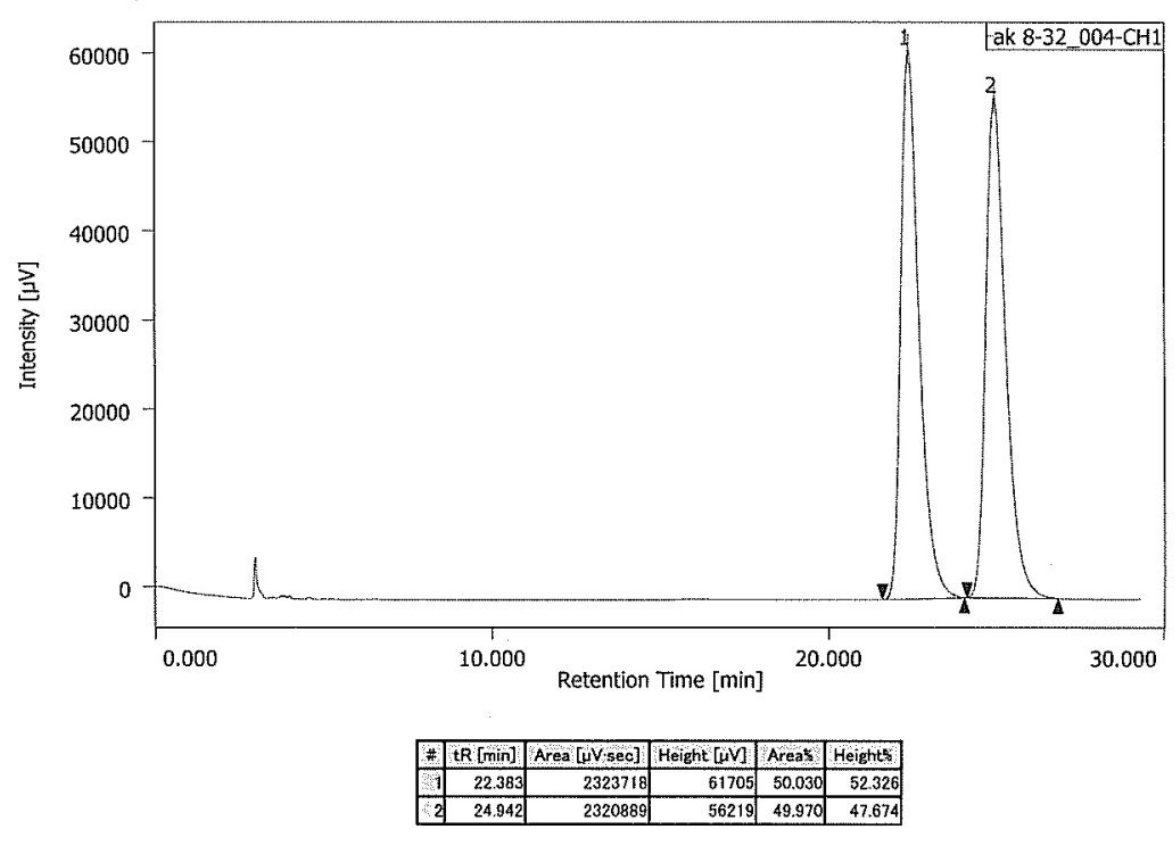

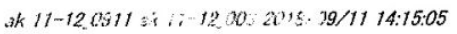

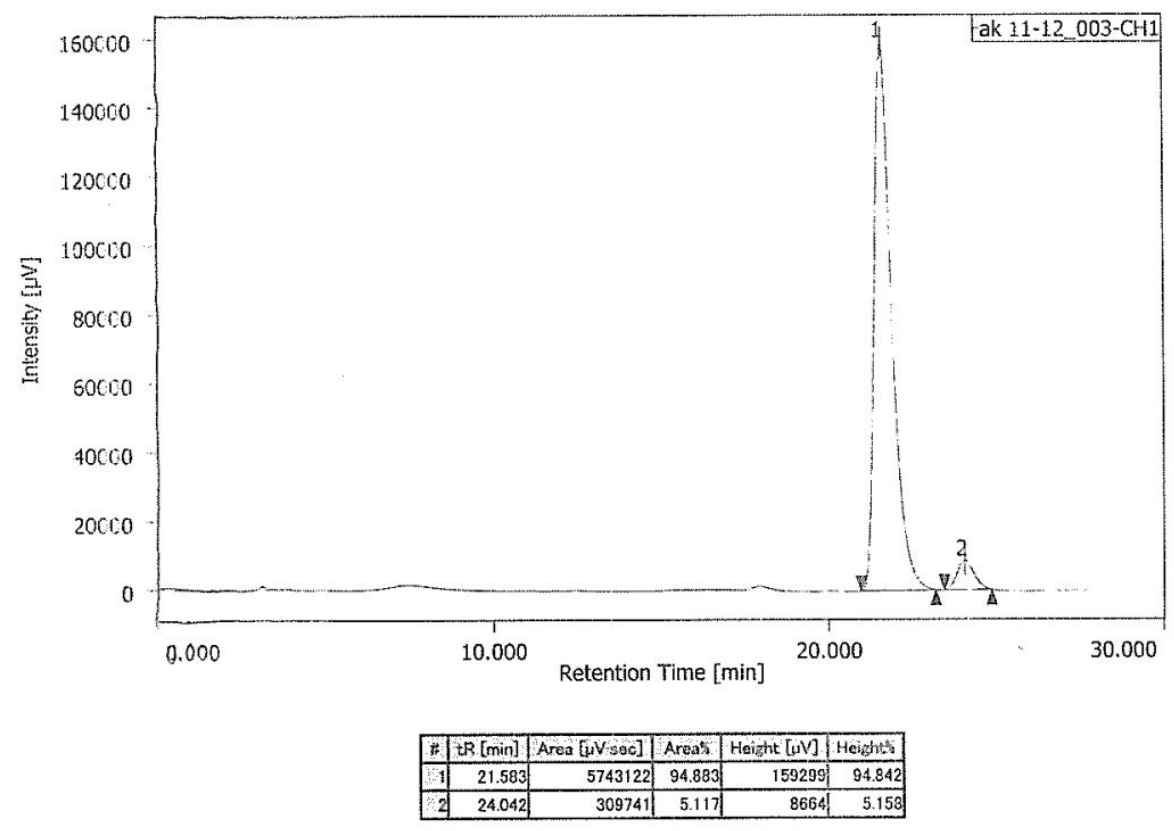


Supporting Information

$3 b$

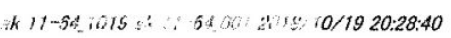<smiles>CCC(C)N1C[C@]2(CC1=O)C(C(C)(C)C)=Nc1ccc(Br)cc12</smiles>

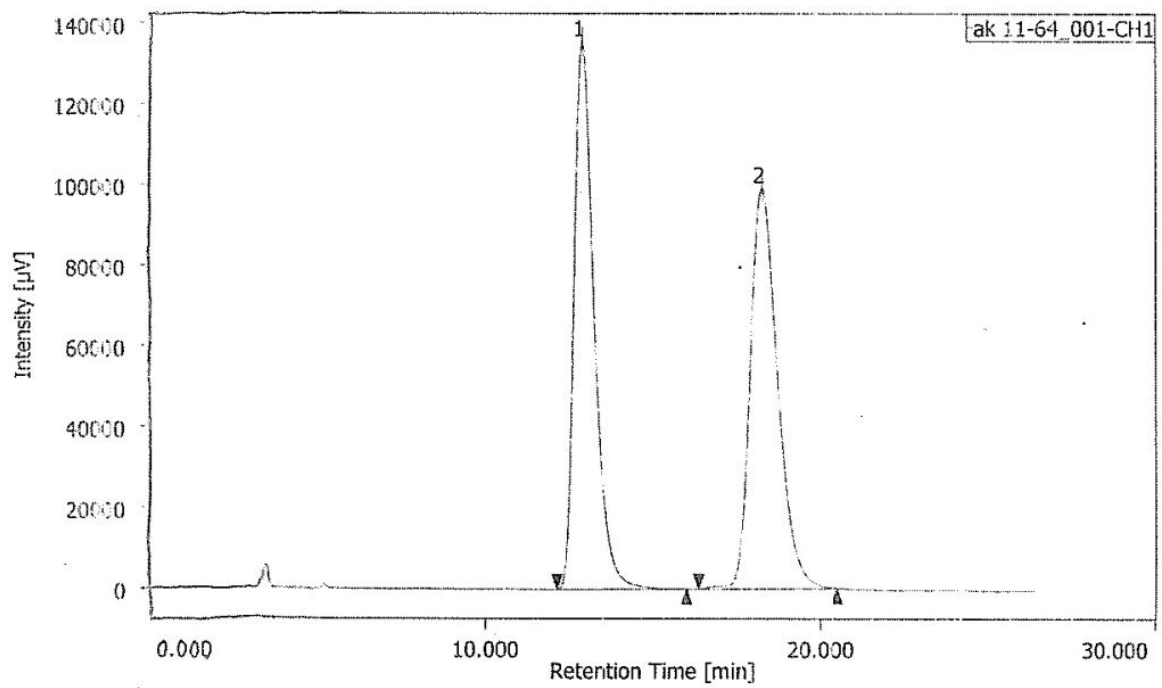

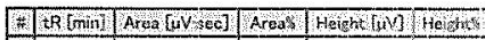

\begin{tabular}{|l|r|r|r|r|r|}
\hline 1 & 12.908 & 5607459 & 50.015 & 135582 & 57.767 \\
\hline 2 & 18.258 & 5604146 & 49.985 & 99125 & 42.233 \\
\hline
\end{tabular}

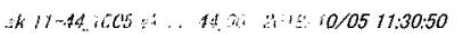

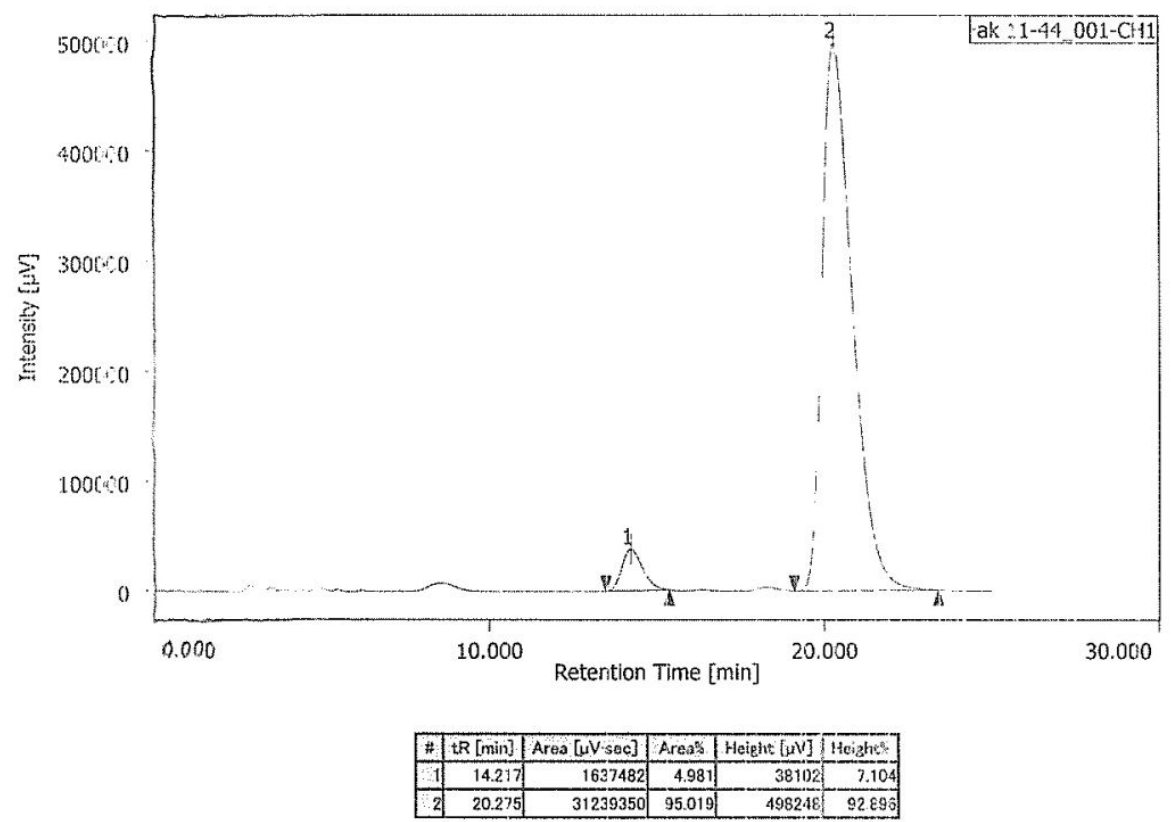


Supporting Information

$3 c$

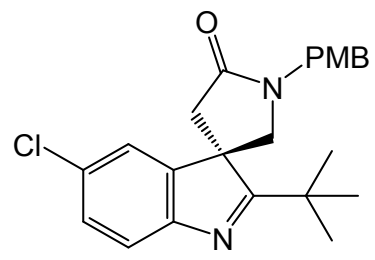

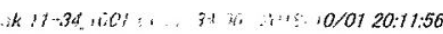

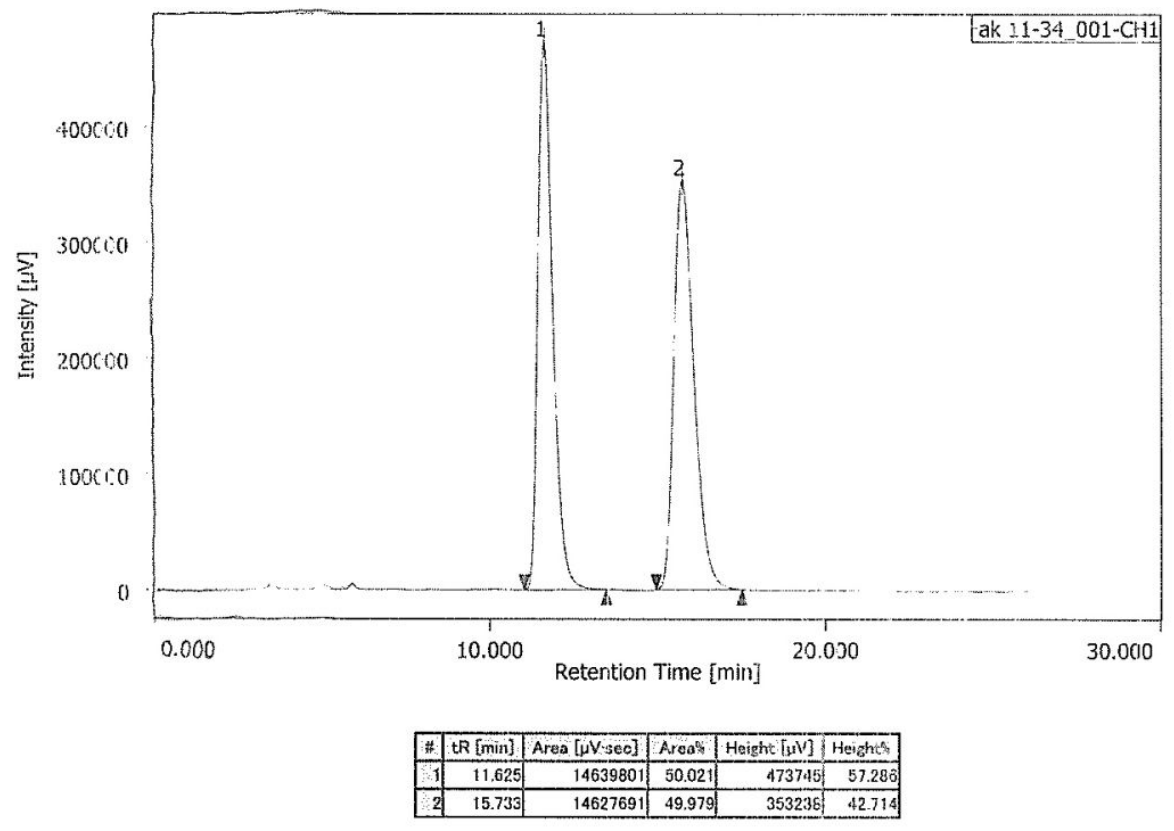

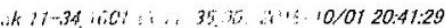

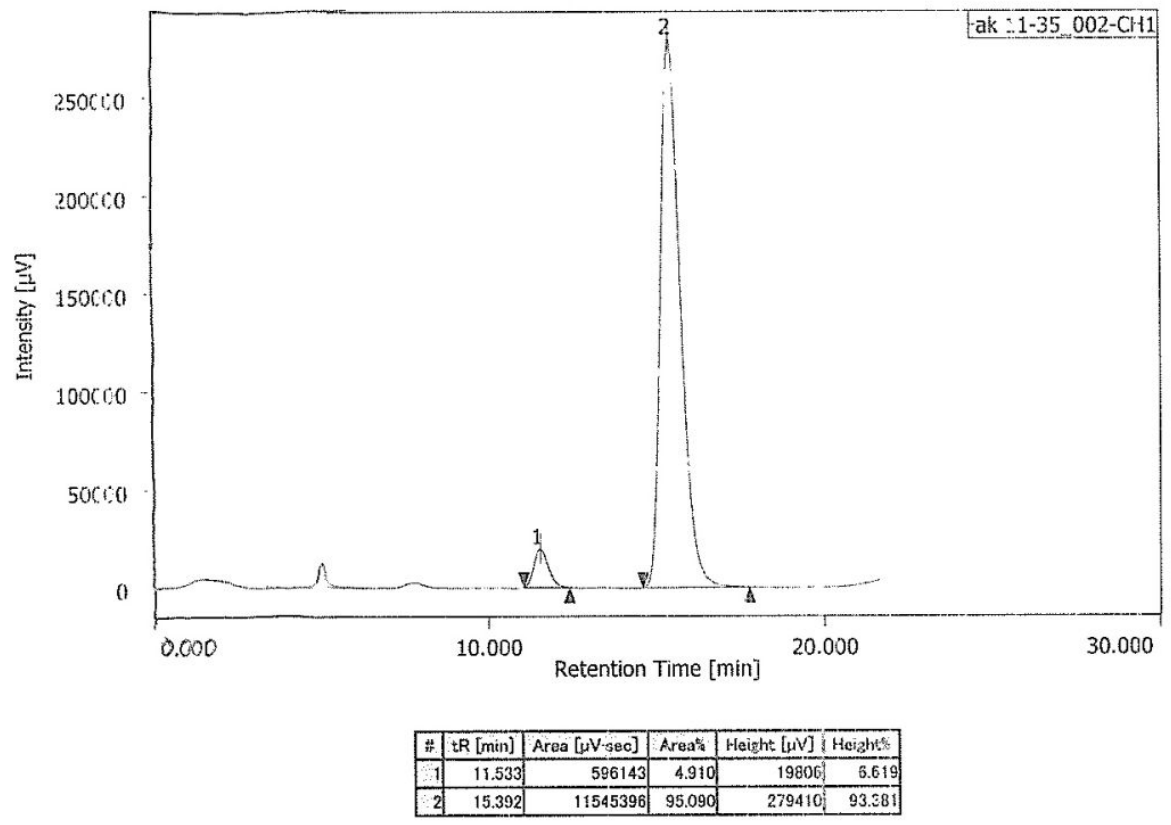


Supporting Information

$3 d$

ju-0724_0724004 2019/07/24 17:10:10
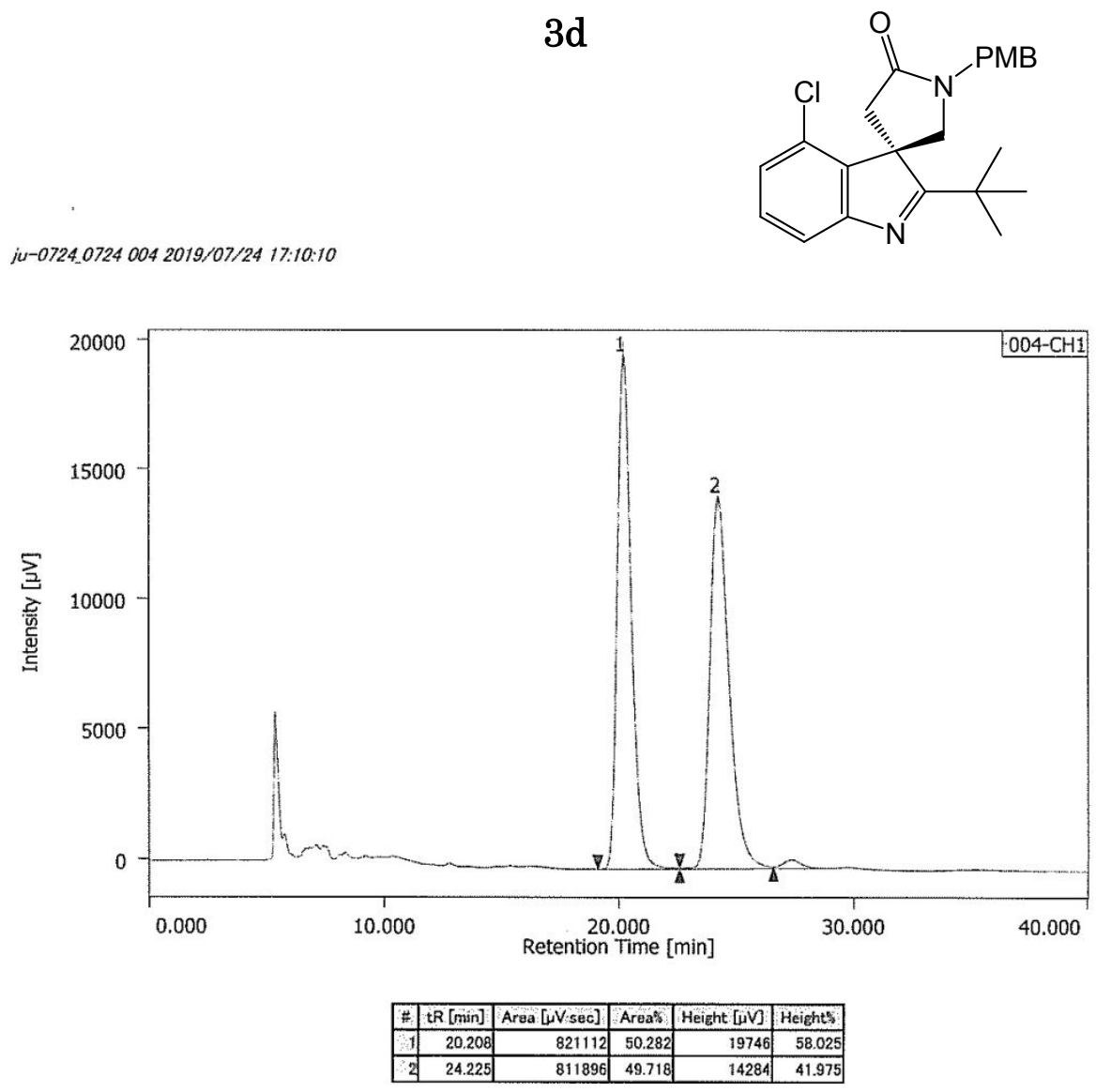

$j u-0724 \_0724005$ 2019/07/24 18:19:16

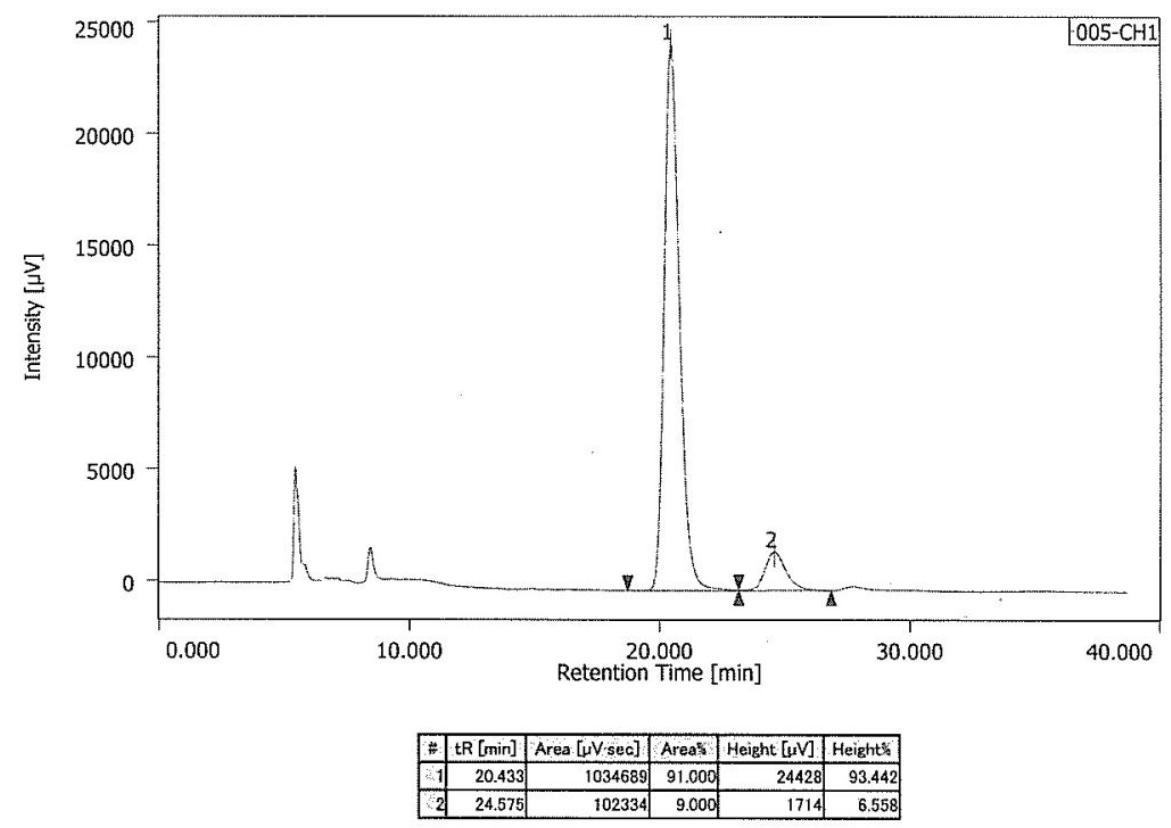


Supporting Information

ak 12-30,0107 ak 12-30_004 2019/01/07 13:44:55

$3 e$

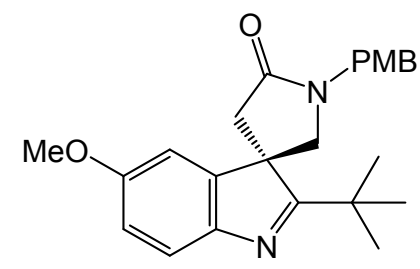

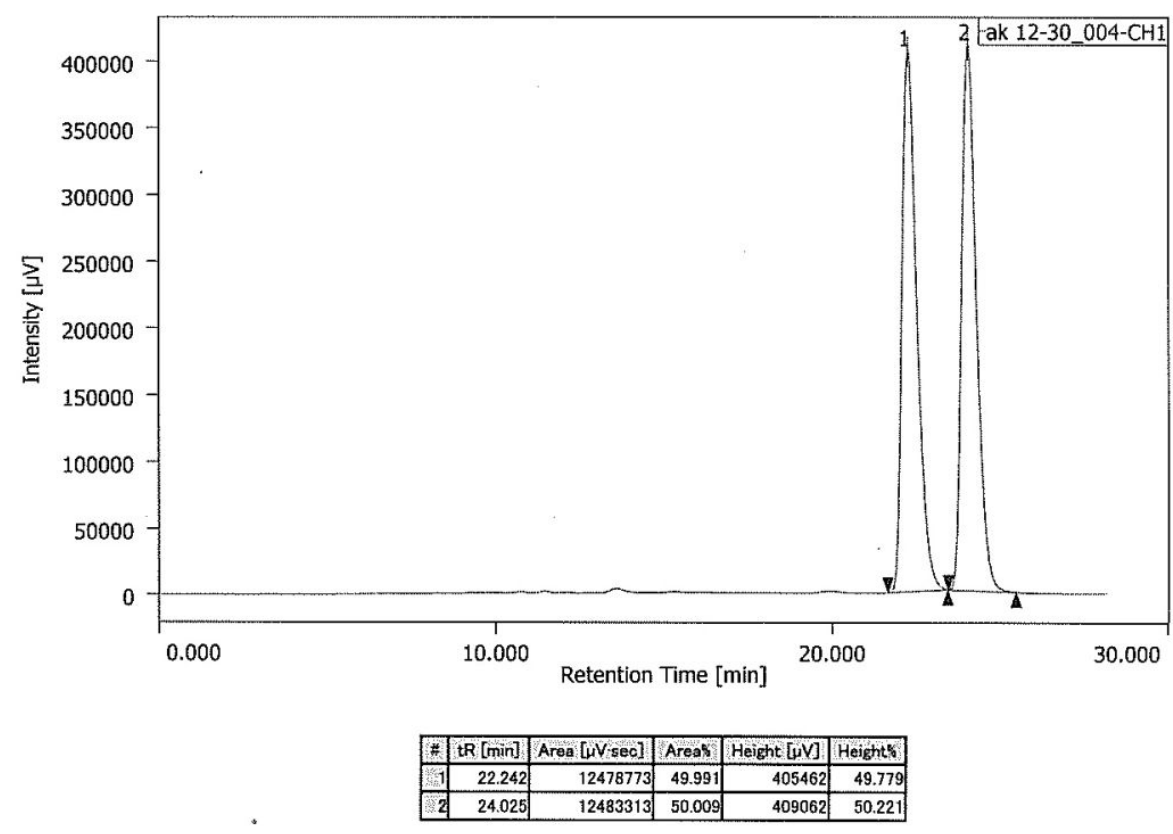

ak 12-30_0107 ak 12-31_006 2019/01/07 13:45:37

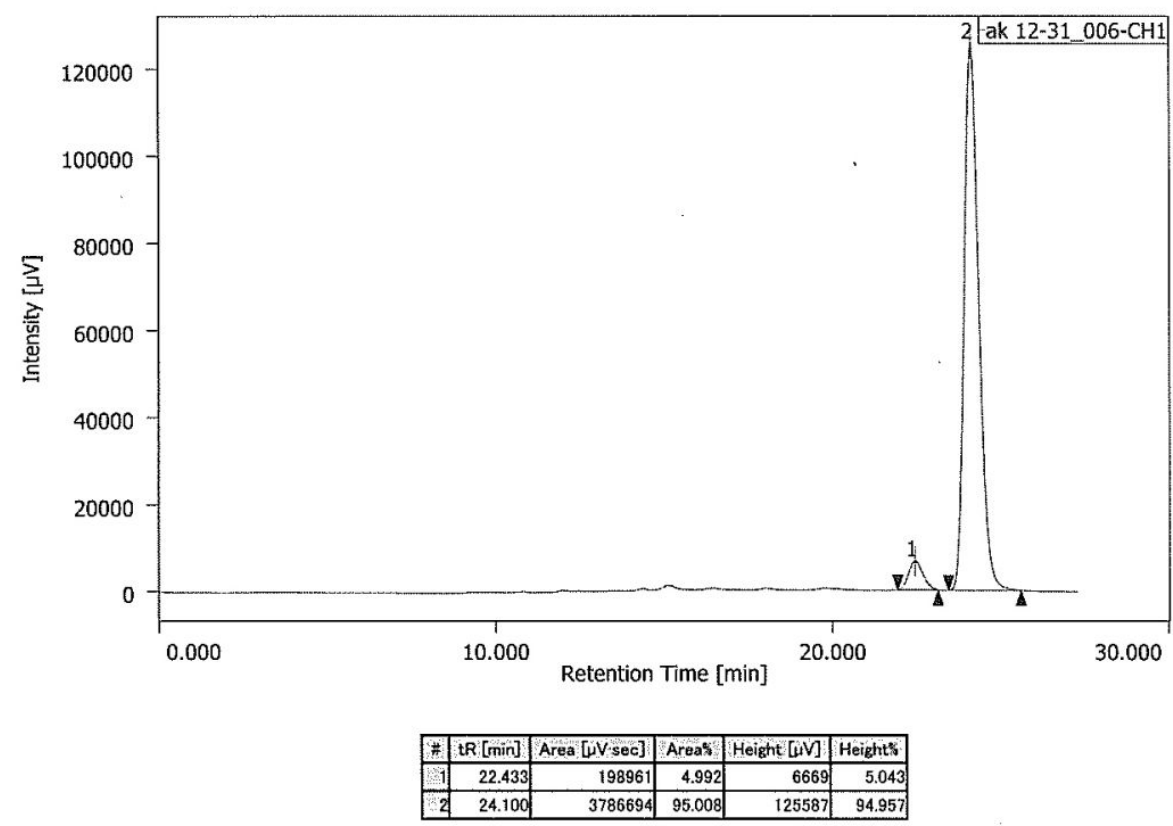


Supporting Information

3f

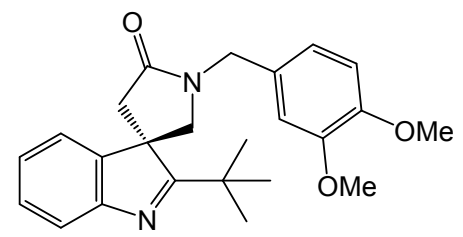

ju-0228_0228 001 2020/02/28 17:51:24

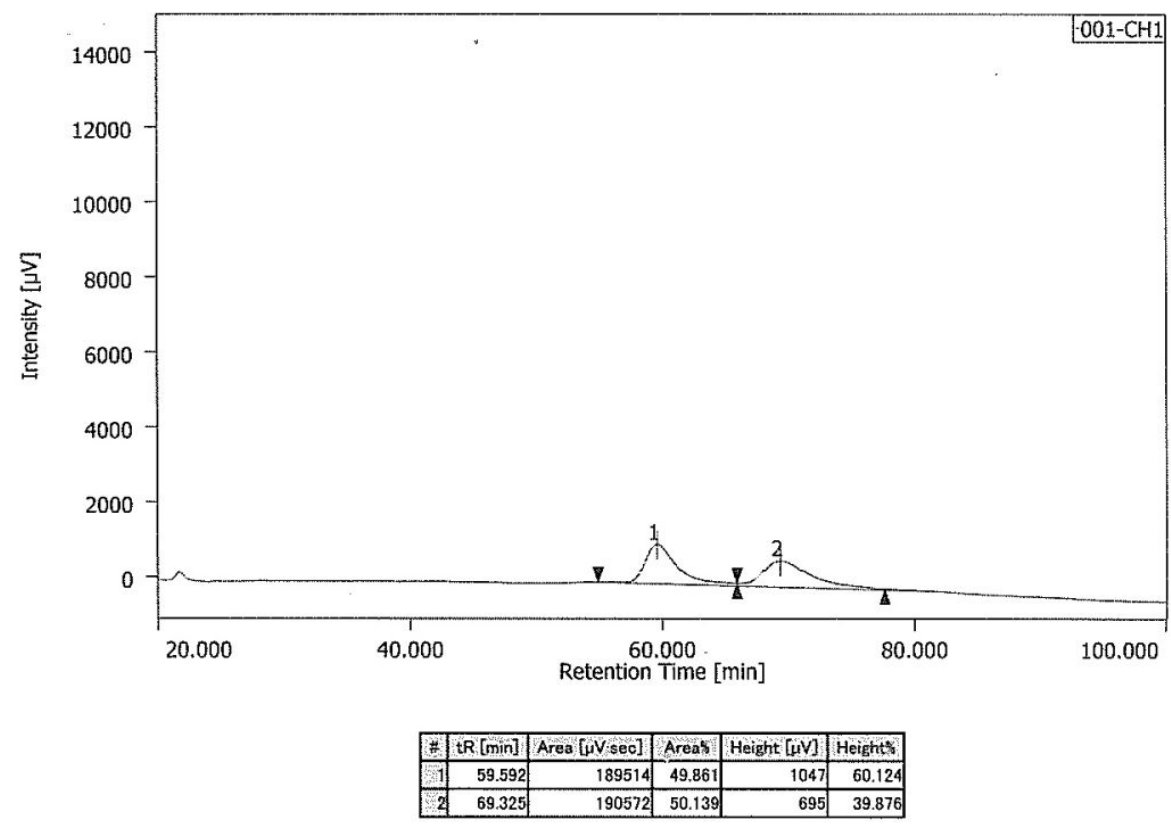

ju-0220_0220 003 202a/02/21 3:21:28

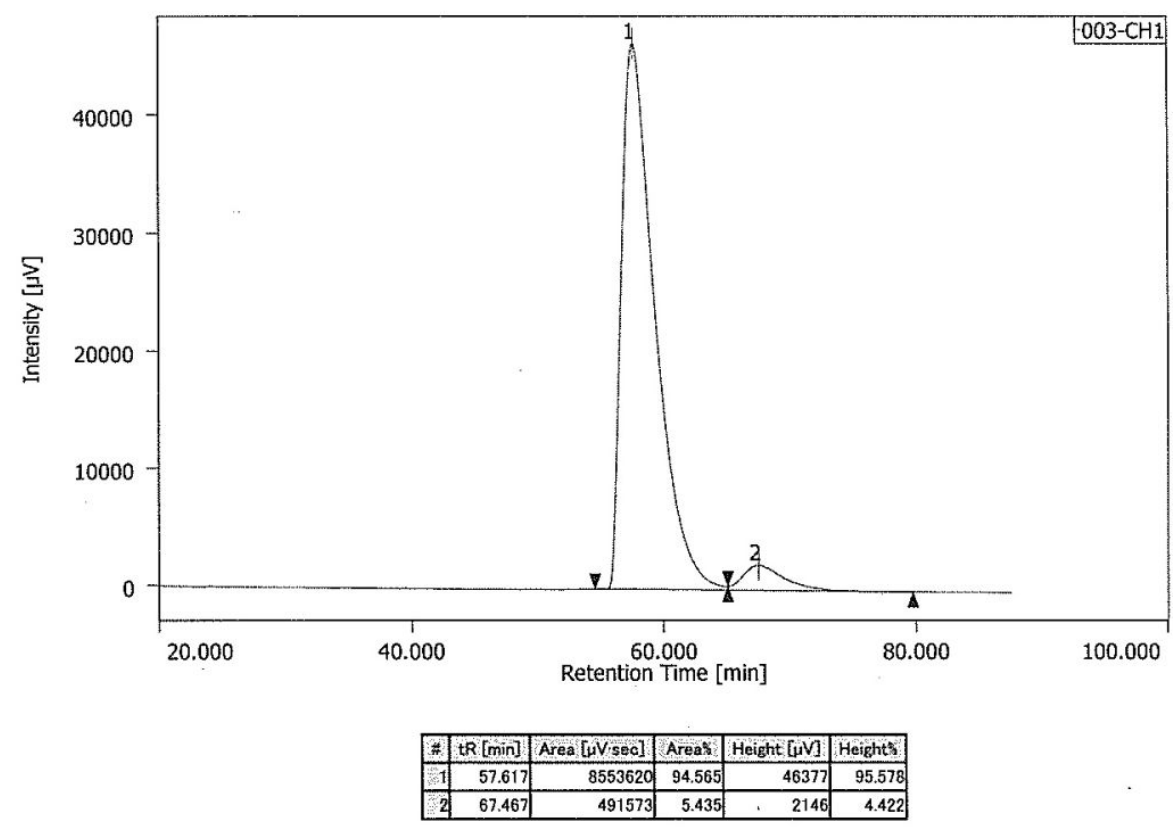


Supporting Information

ak 12-12.1121 ak 12-12.001 2018/11/21 12:49:50
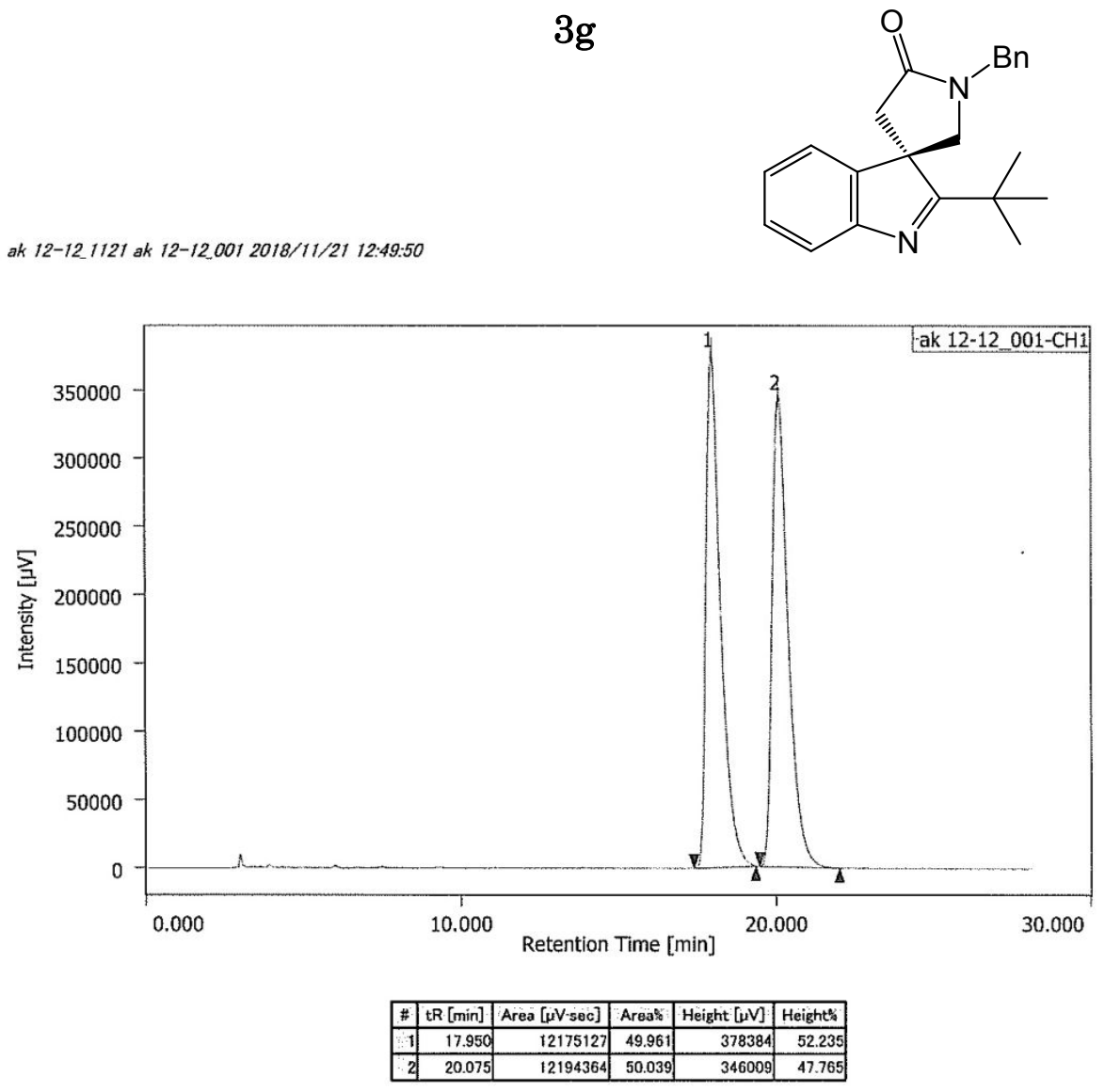

ak 12-12,1121 ak 12-13_003 2018/11/21 12:51:32

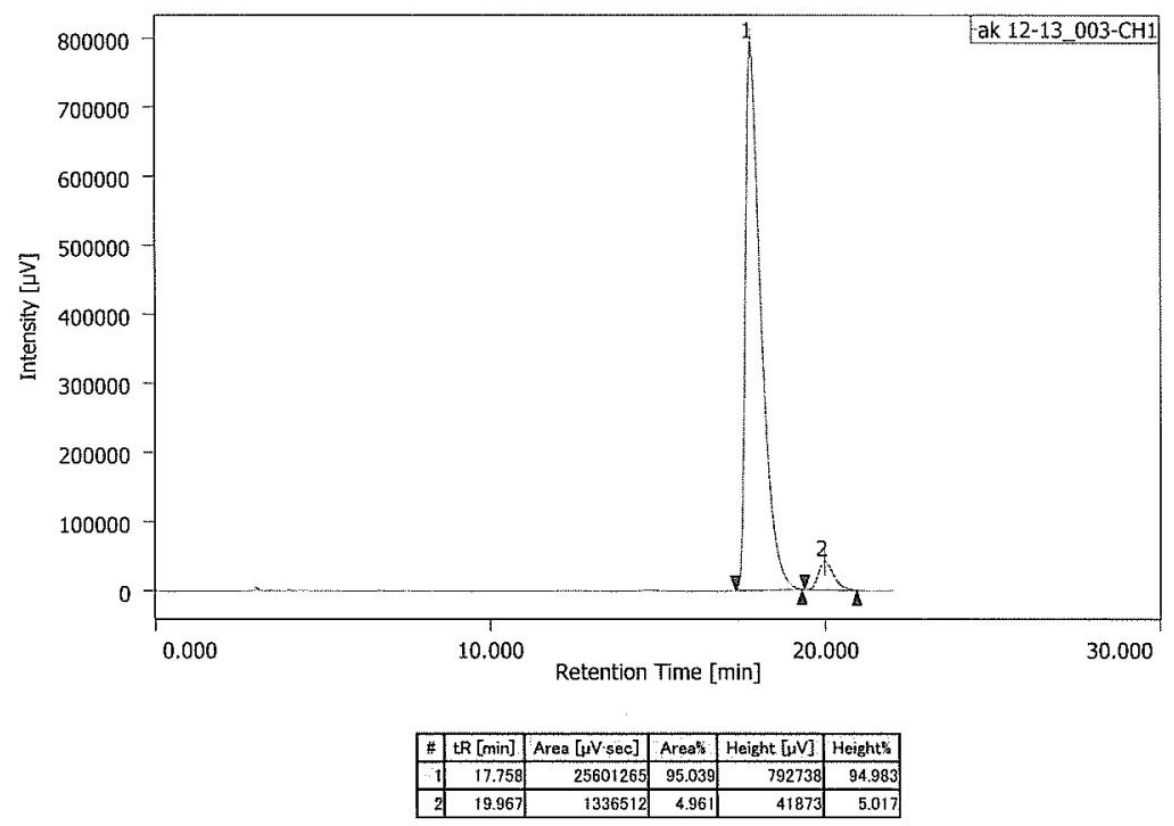


Supporting Information

$3 \mathrm{~h}$

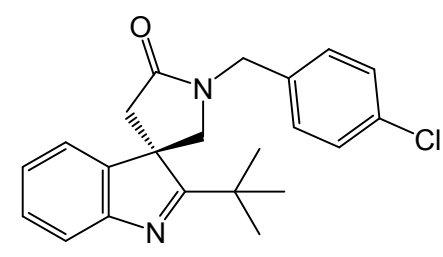

ju-0303_0303 001 2020/03/03 17:31:03

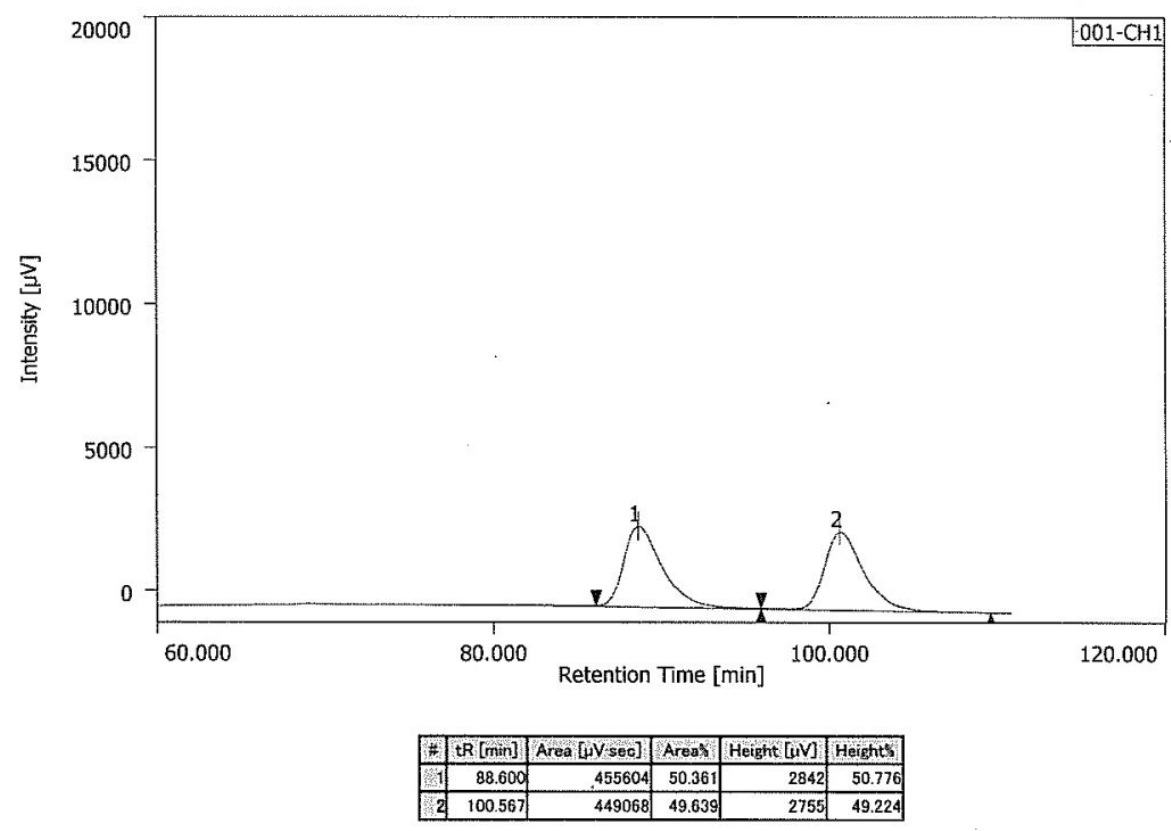

ju-0302.0302 001 2020/03/03 17:33:30

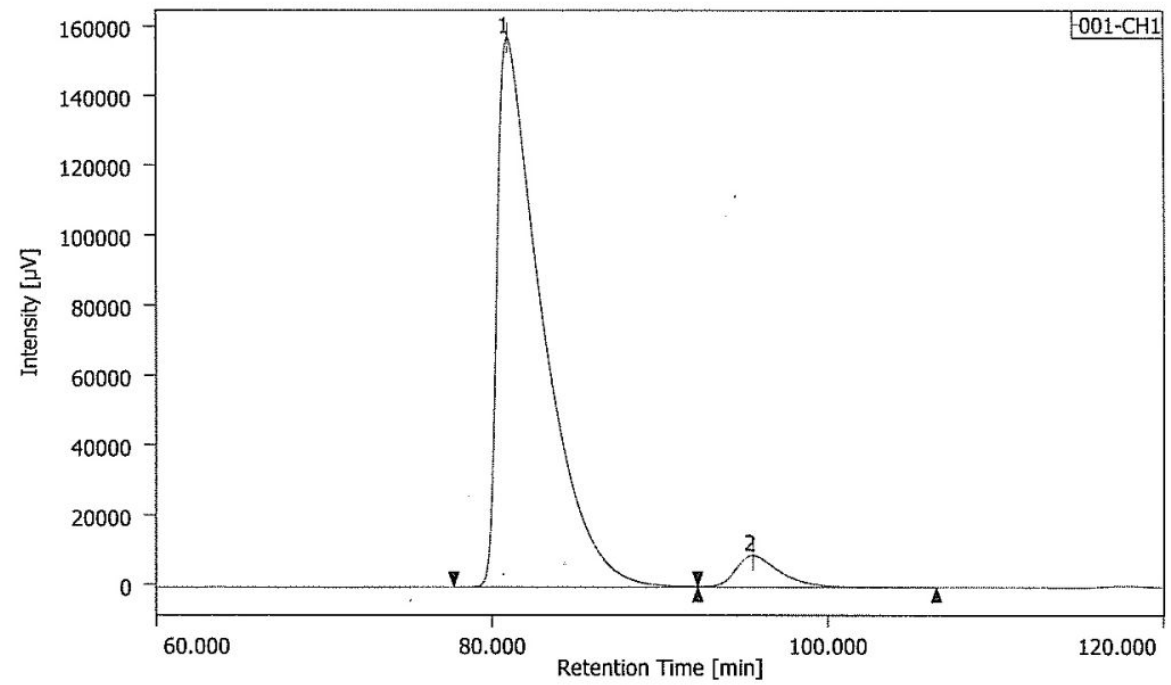

\begin{tabular}{|l|l|l|l|l|l|}
\hline$\#$ tR [min] & Area [ijV sec] & Areas & Height [iv] & Heights \\
\hline
\end{tabular}

\begin{tabular}{|r|r|r|r|r|r|}
\hline 1 & 80.900 & 29265634 & 94.544 & 157317 & 94.520 \\
\hline 2 & 95.50 & 168833 & 5.45 & 9121 & 5.40 \\
\hline
\end{tabular}


Supporting Information

$3 \mathbf{i}$

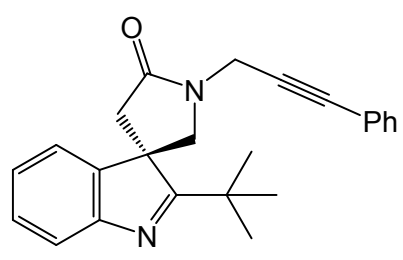

$j u-0303.0303004$ 2020/03/03 23:08:15

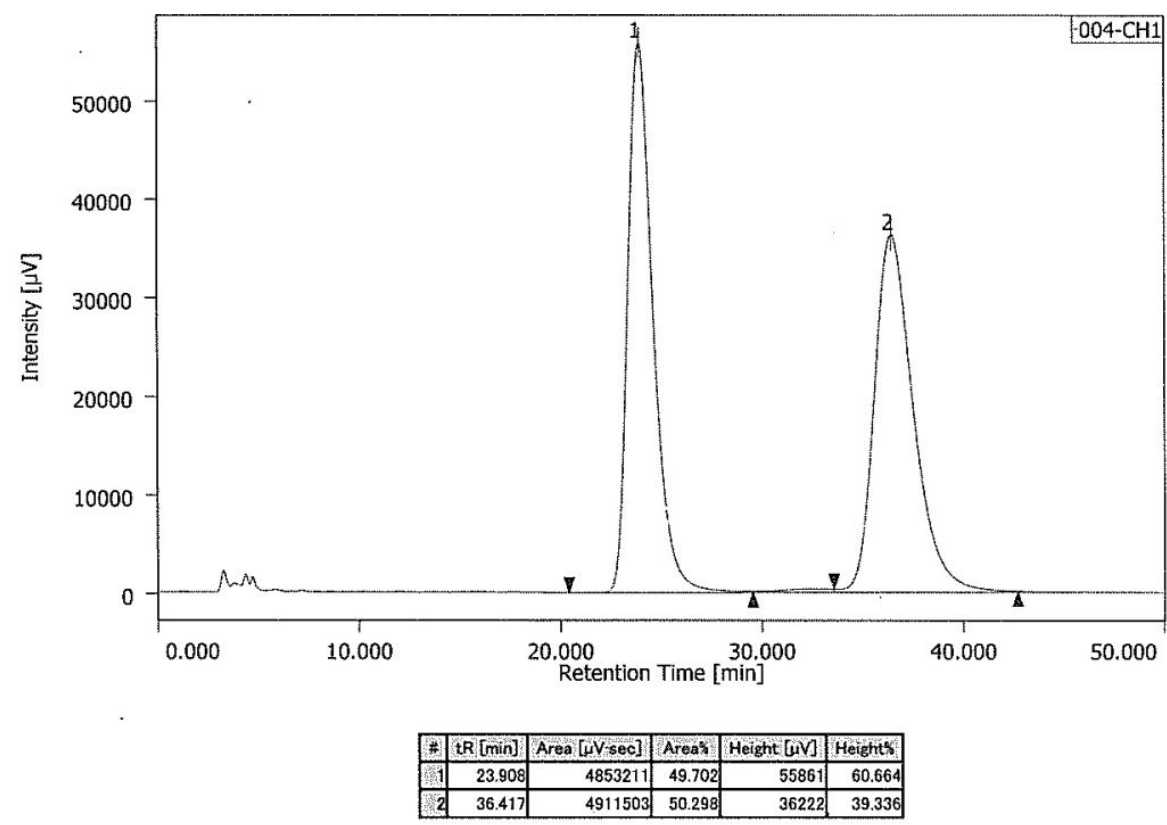

$i n-0303.03030032020 / 03 / 03$ 23:08:21

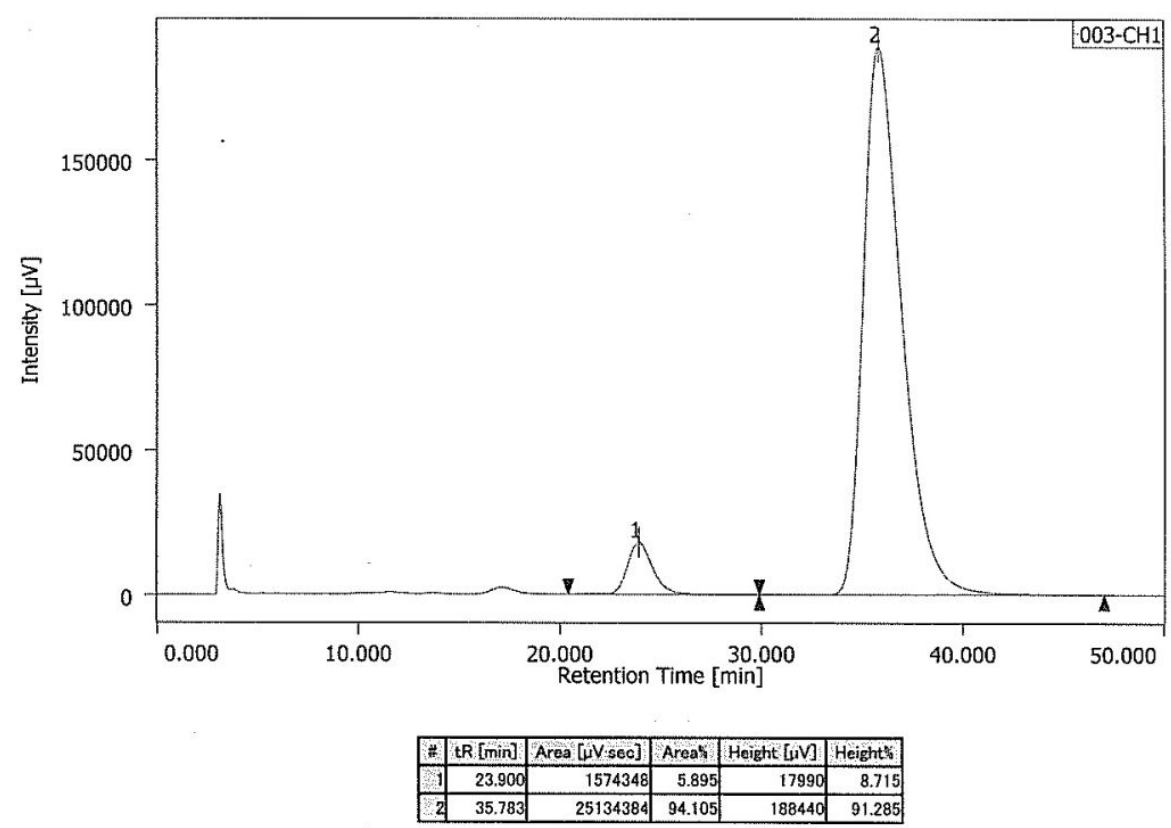


Supporting Information

ak 11-68_1031 ak 11-68_003 2018/11/01 16:25:12
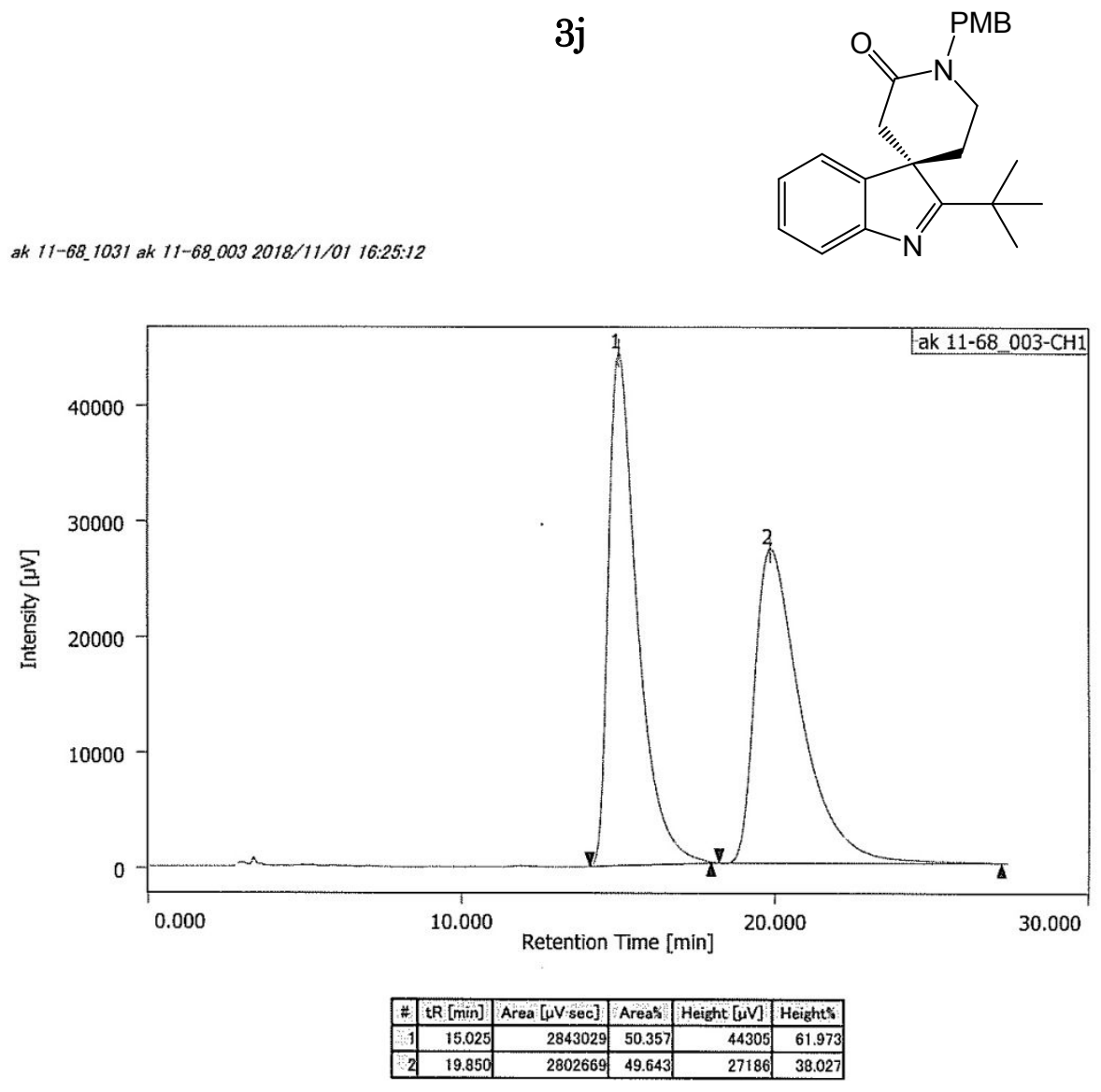

ak 11-76_1101 ak 11-69_004 2018/11/01 16:22:40

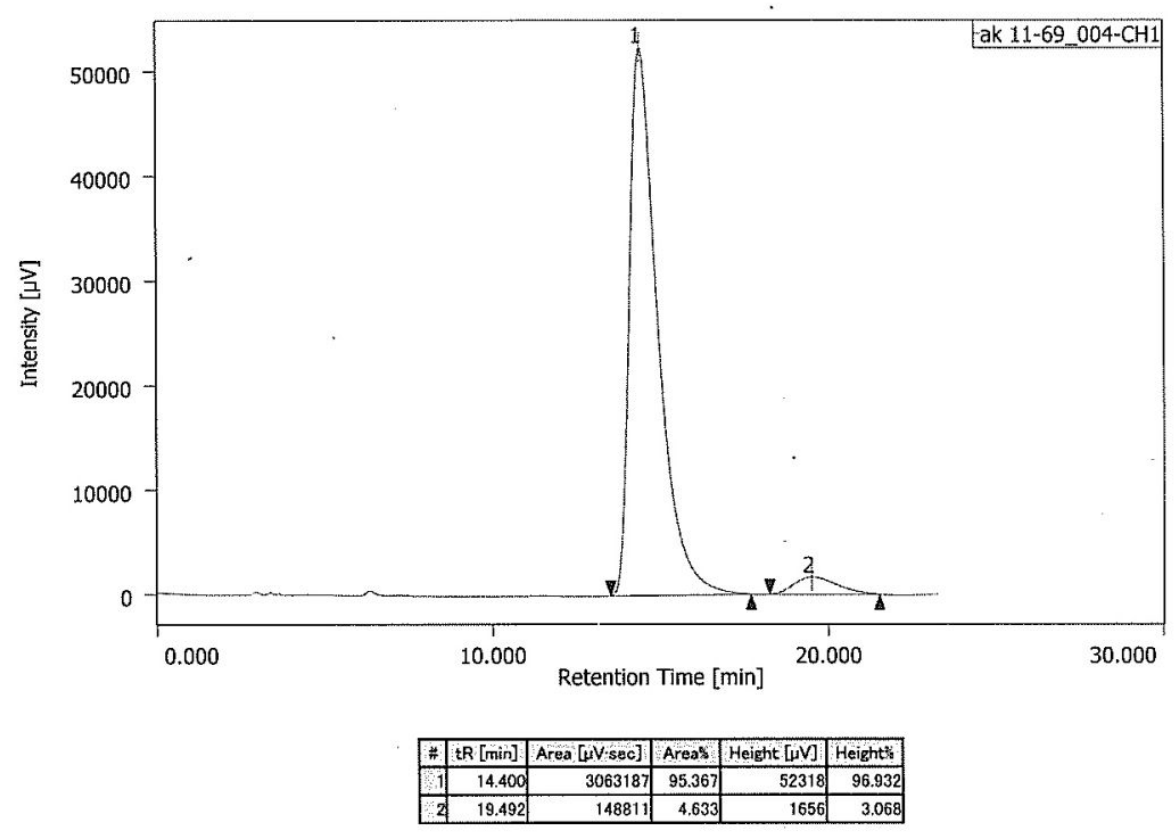


Supporting Information

$3 \mathbf{k}$<smiles>CCCCN1C[C@]2(CC1=O)CC(c1ccccc1)=Nc1ccccc12</smiles>

ak 5-64_0807 ak 5-64_002 2018/12/18 21:27:16

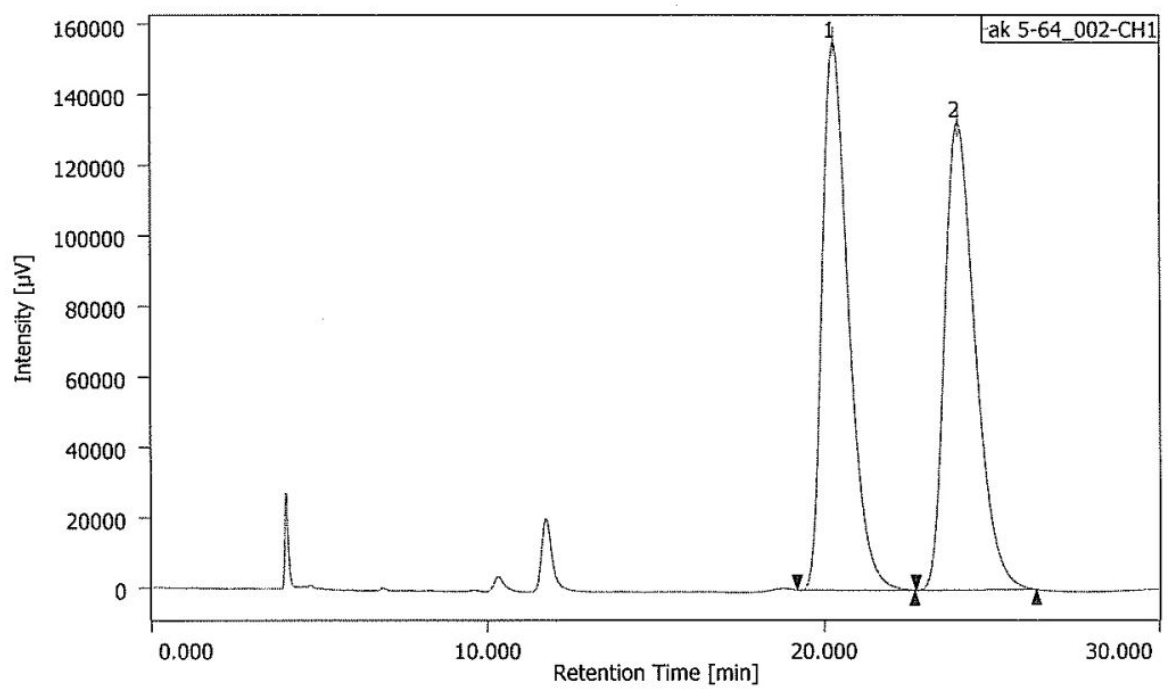

\begin{tabular}{|r|r|r|r|r|r|}
\hline$\#$ & \multicolumn{1}{|c|}{$R[\mathrm{~min}]$} & Area $[\mu \mathrm{V}$ sec $]$ & Height $[\mu \mathrm{V}]$ & Areas & Heights: \\
\hline 1 & 20.283 & 8439506 & 155372 & 49.997 & 53.941 \\
\hline
\end{tabular}

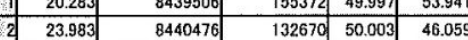

ak $11-14$ _o:13 si i, 14:00:20!s $99 / 13$ 20:13:35

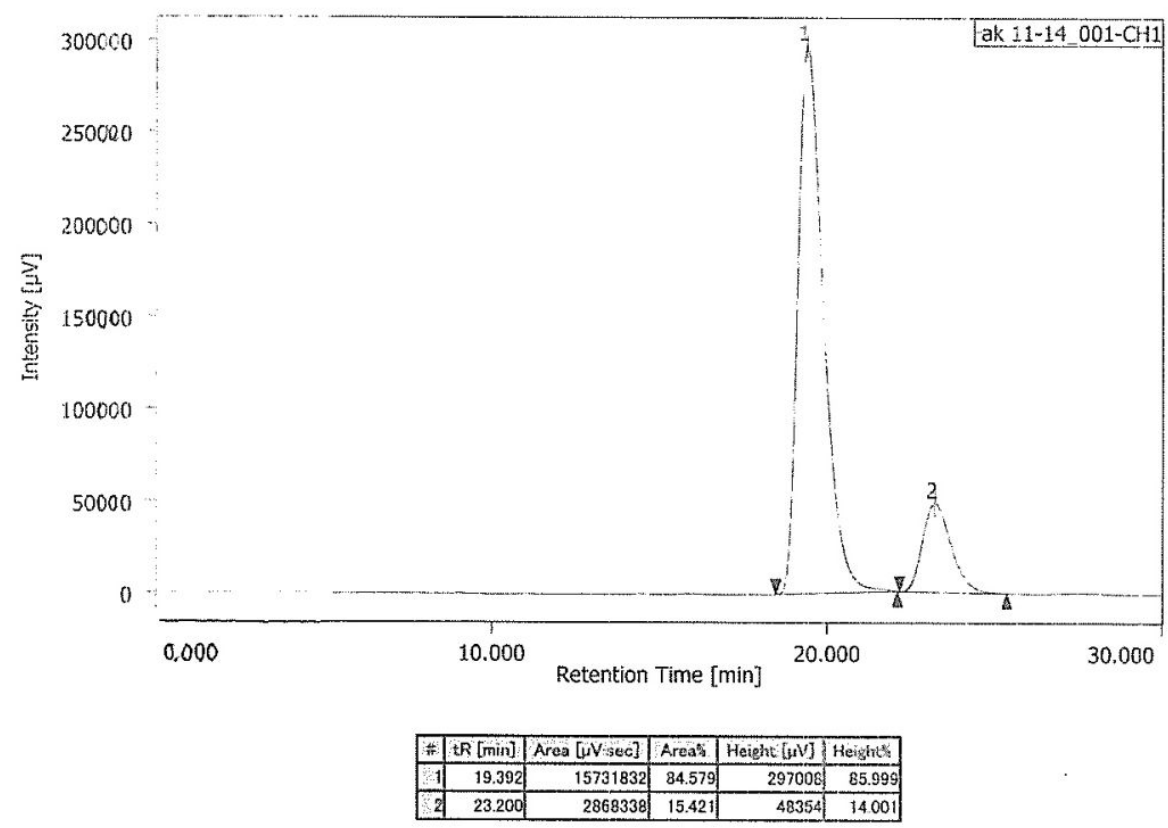


Supporting Information

31

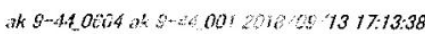
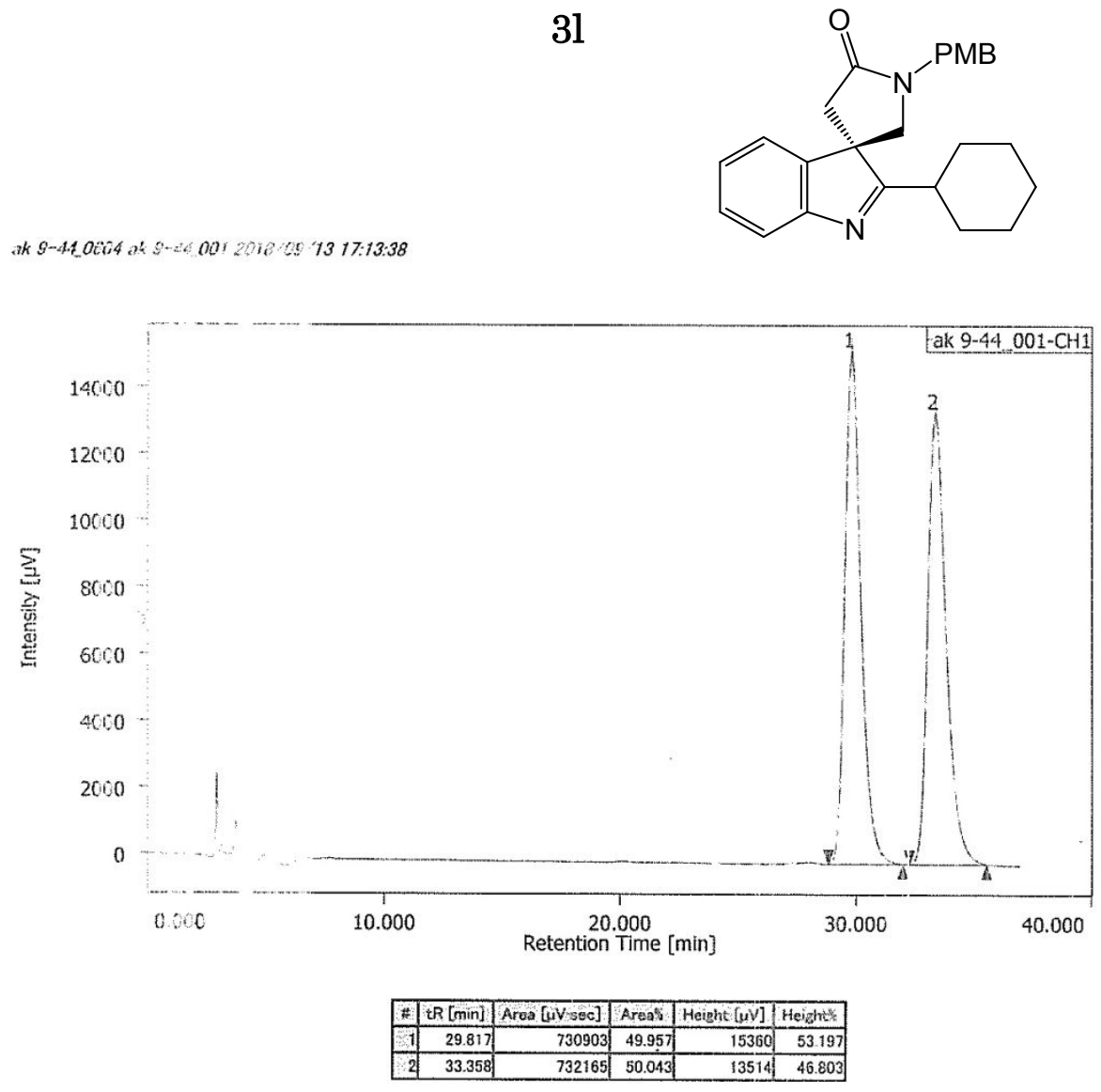

ak $11-15,0313$ an $\quad$ is $-15,00: 2015,19,13$ 17:14:40

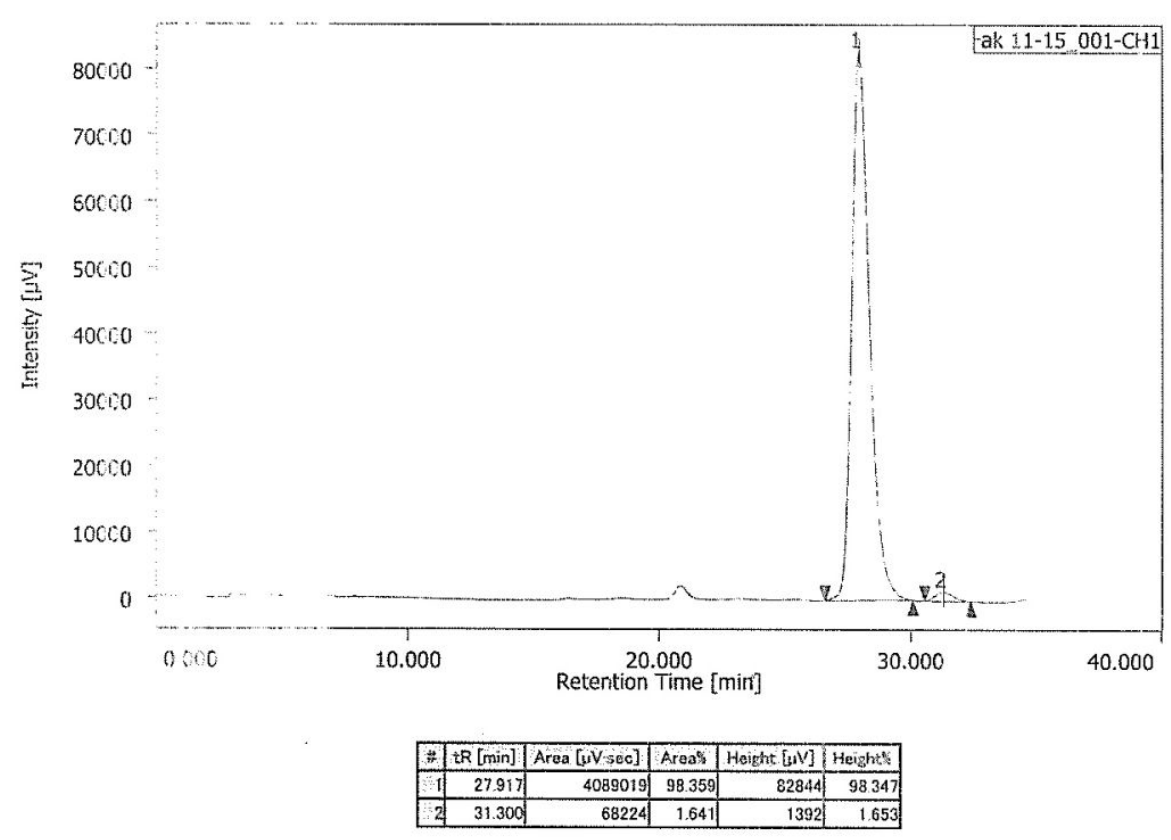


Supporting Information

$3 \mathrm{~m}$

ak 11-76_1101 ak 11-76_003 2018/11/101 18:12:13<smiles>C=C(C)CN1C[C@]2(CC1=O)C(C(C)(C)C)=Nc1ccccc12</smiles>

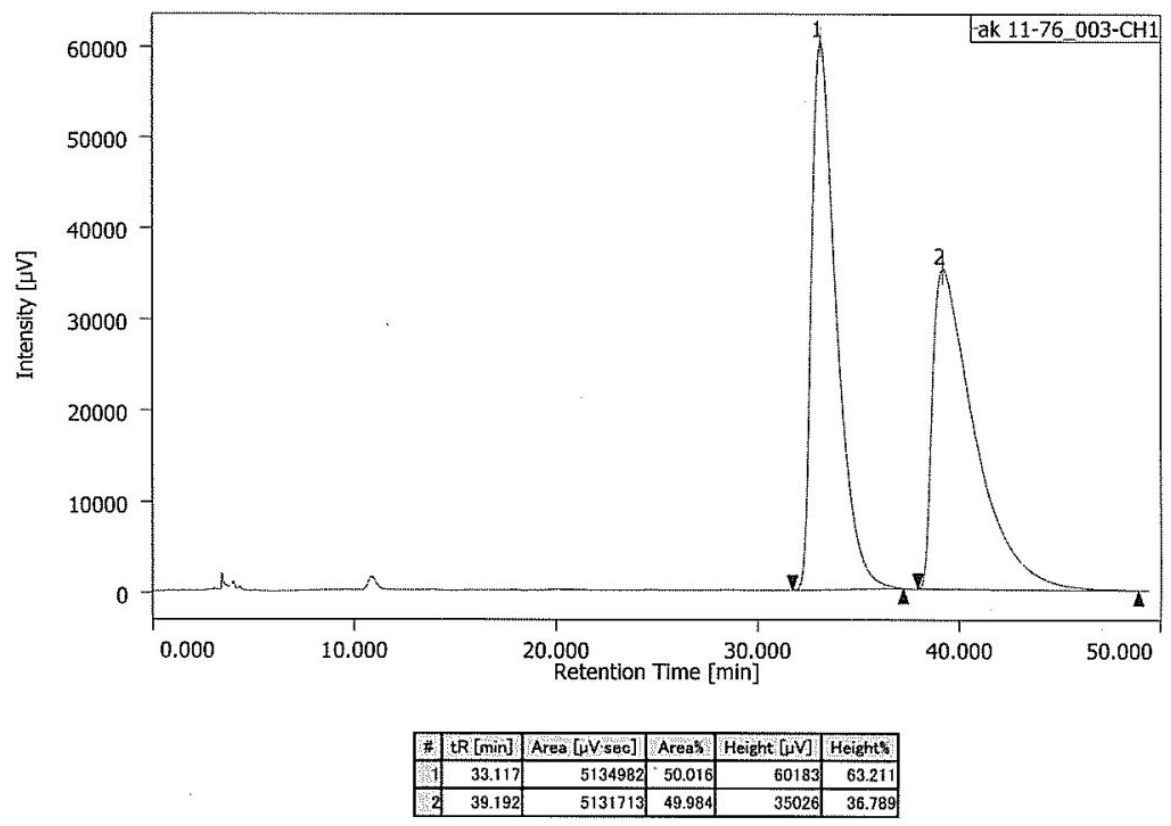

ak 11-76_1101 ak 11-77_005 2018/11/01 18:11:58

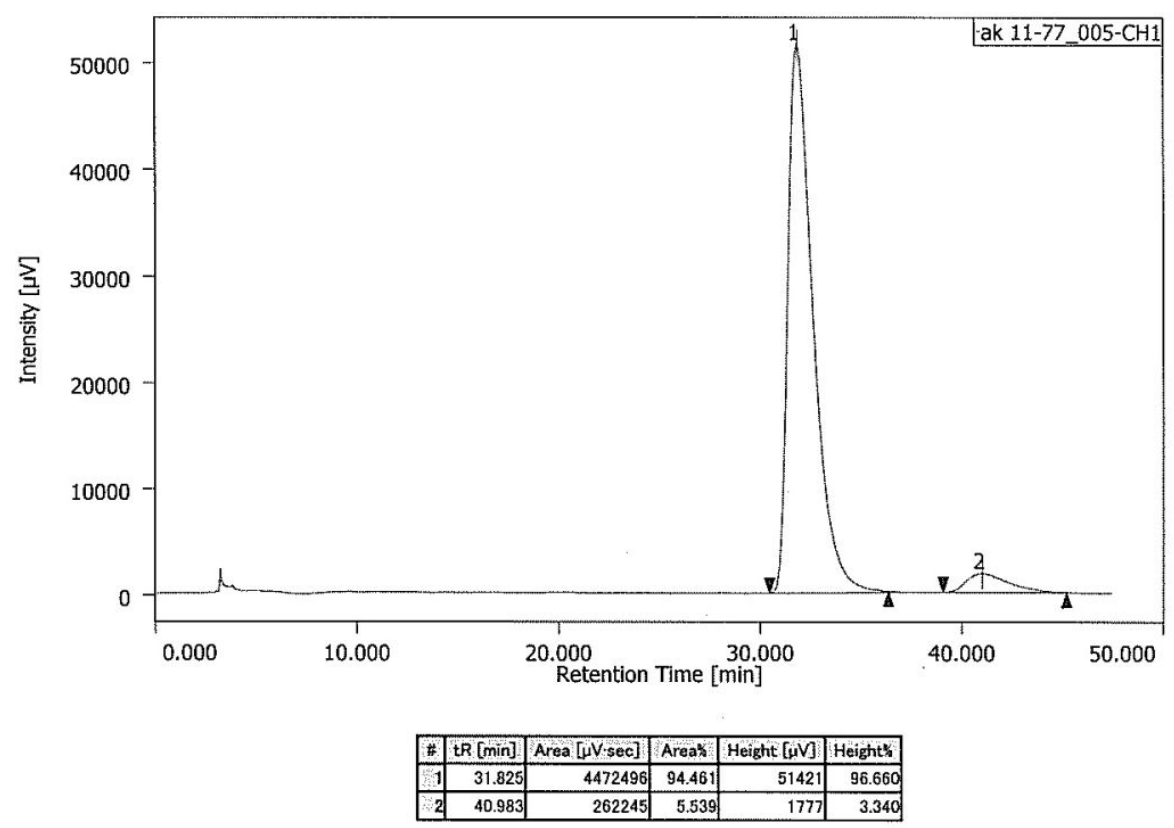


Supporting Information

$3 n$<smiles>CCCCN1C[C@]2(CC1=O)C(C)=Nc1ccccc12</smiles>

ju-0414_0414004 2020/04/14 16:26:43

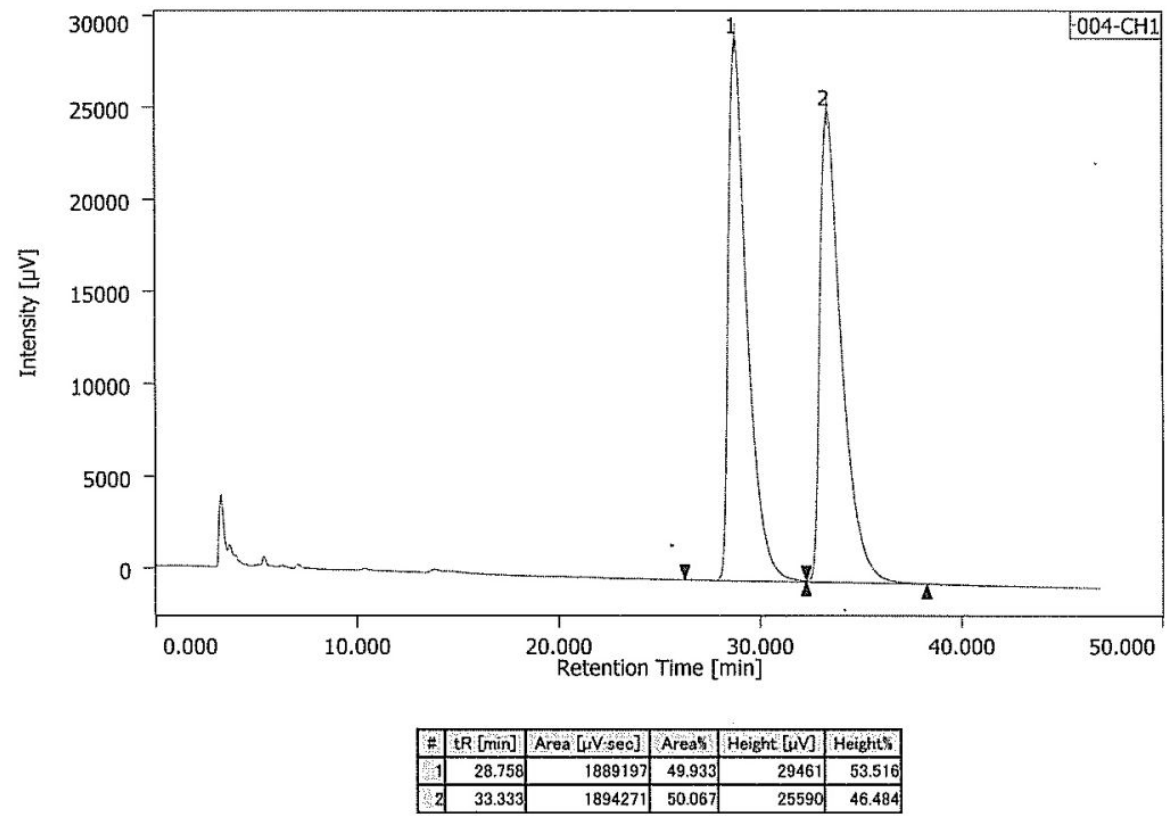

$j u-0414 \_0414005$ 2020/04/14 16:26:33

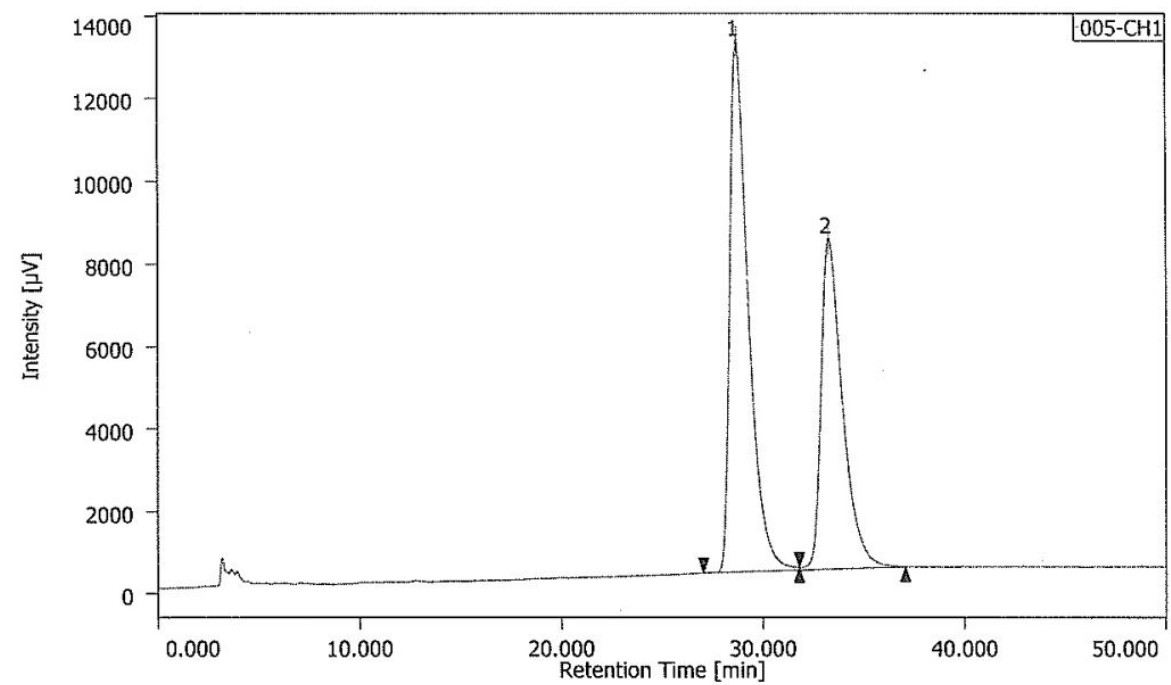

\begin{tabular}{|l|l|l|l|l|l|}
\hline$\#$ tR $[$ min $]$ & Area $[\mu \mathrm{V}$-sec $]$ & Areas & Height $[\mu \mathrm{V}]$ & Heights \\
\hline
\end{tabular}

\begin{tabular}{|r|r|r|r|r|r|}
\hline 1 & 28.692 & 823049 & 58.180 & 12879 & 61.567 \\
\hline 2 & 33.242 & 591608 & 41.820 & 8040 & 38.433 \\
\hline
\end{tabular}


Supporting Information

11

ju-1220 $12200012020 / 05 / 18$ 18:58:18
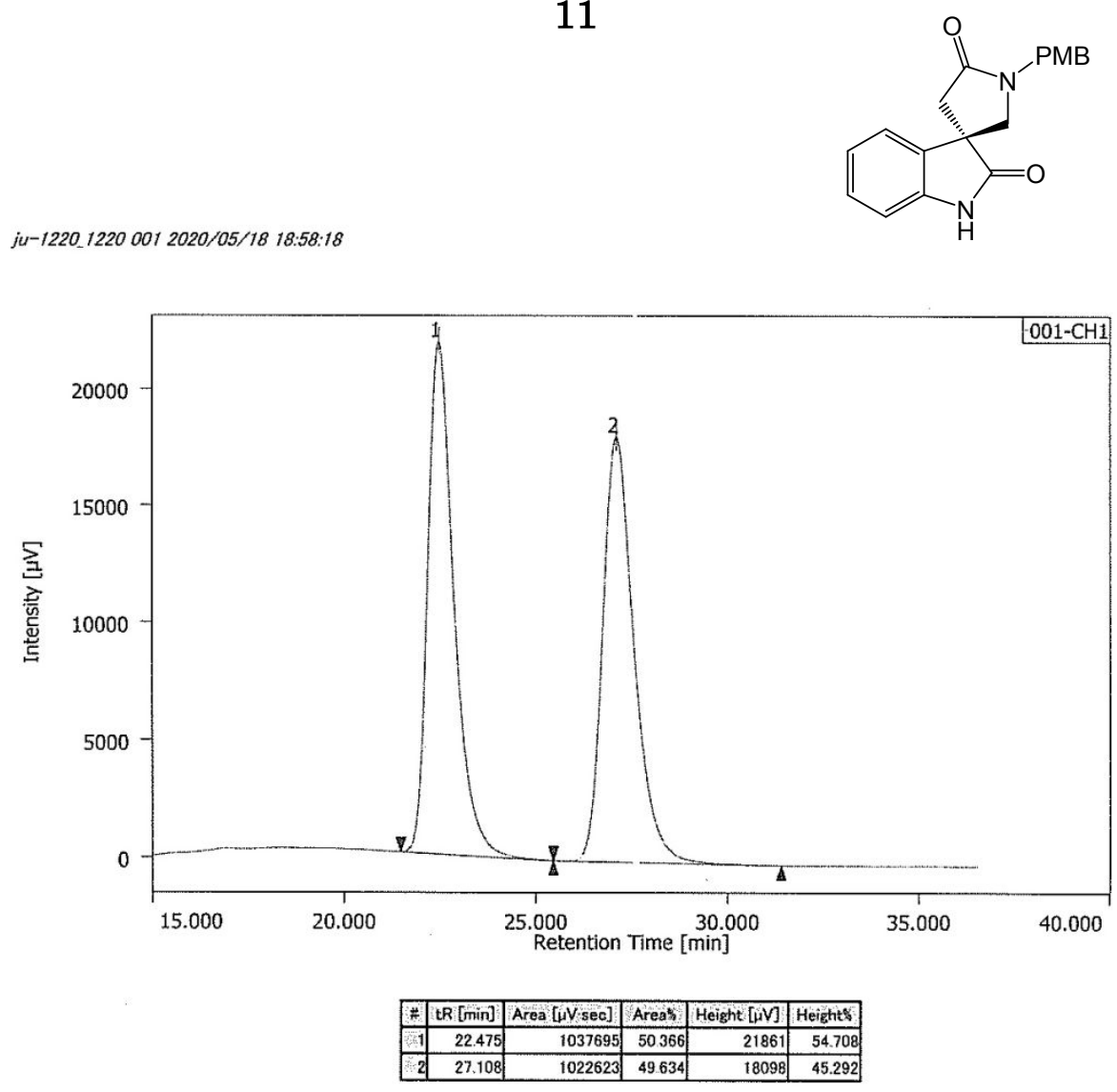

$j u-1220,1220002$ 2020/05/18 18:58:24

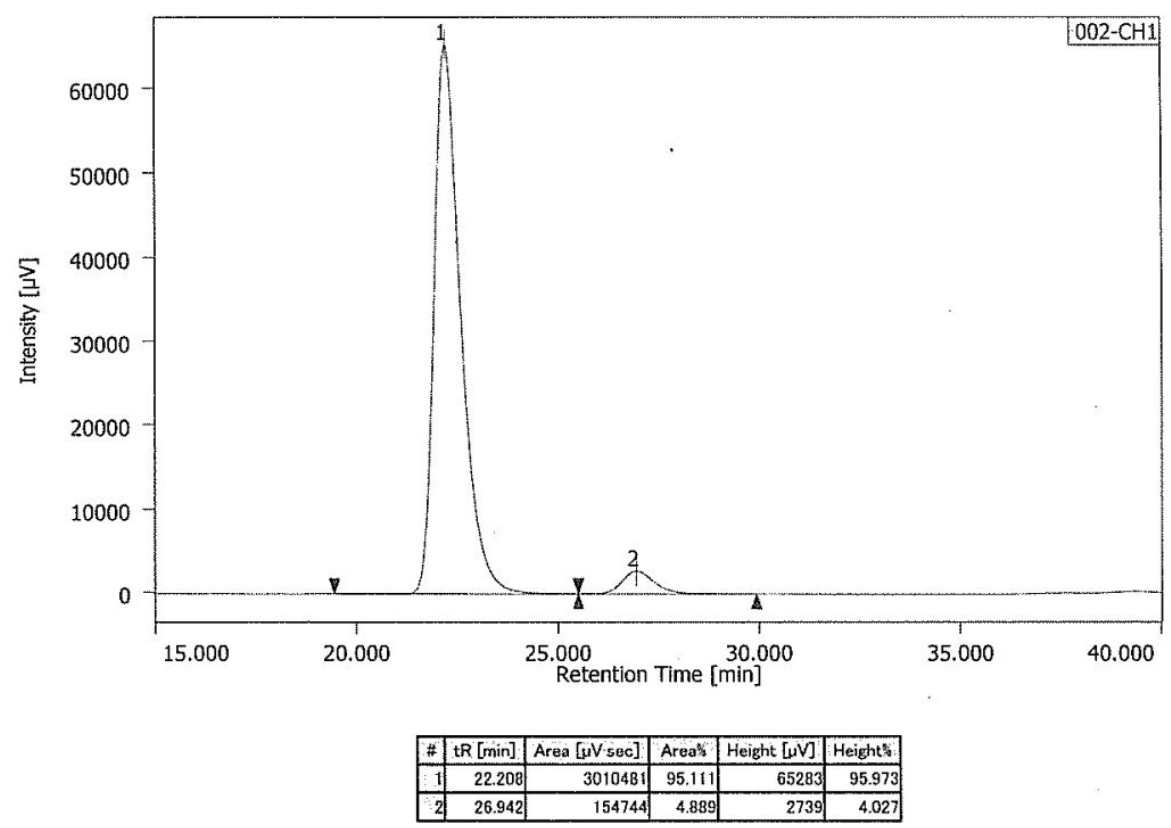




\section{8. $\underline{\text { References }}$}

[1] Reaction data of phenol derivatives, see; Nakayama, H.; Harada, S.; Kanda, A.; Kwok, I. M.-Y.; Nemoto, T. Binary Additive Effect of Benzoic Acid in ipso-FriedelCrafts-Type Dearomatization of Phenols Using a Chiral Silver Phosphate. Tetrahedron 2018, 74, 2435-2439.

[2] Gaussian 16, Revision C.01, Frisch, M. J.; Trucks, G. W.; Schlegel, H. B.; Scuseria, G. E.; Robb, M. A.; Cheeseman, J. R.; Scalmani, G.; Barone, V.; Petersson, G. A.; Nakatsuji, H.; Li, X.; Caricato, M.; Marenich, A. V.; Bloino, J.; Janesko, B. G.; Gomperts, R.; Mennucci, B.; Hratchian, H. P.; Ortiz, J. V.; Izmaylov, A. F.; Sonnenberg, J. L.; Williams-Young, D.; Ding, F.; Lipparini, F.; Egidi, F.; Goings, J.; Peng, B.; Petrone, A.; Henderson, T.; Ranasinghe, D.; Zakrzewski, V. G.; Gao, J.; Rega, N.; Zheng, G.; Liang, W.; Hada, M.; Ehara, M.; Toyota, K.; Fukuda, R.; Hasegawa, J.; Ishida, M.; Nakajima, T.; Honda, Y.; Kitao, O.; Nakai, H.; Vreven, T.; Throssell, K.; Montgomery, J. A., Jr.; Peralta, J. E.; Ogliaro, F.; Bearpark, M. J.; Heyd, J. J.; Brothers, E. N.; Kudin, K. N.; Staroverov, V. N.; Keith, T. A.; Kobayashi, R.; Normand, J.; Raghavachari, K.; Rendell, A. P.; Burant, J. C.; Iyengar, S. S.; Tomasi, J.; Cossi, M.; Millam, J. M.; Klene, M.; Adamo, C.; Cammi, R.; Ochterski, J. W.; Martin, R. L.; Morokuma, K.; Farkas, O.; Foresman, J. B.; Fox, D. J. Gaussian, Inc., Wallingford CT, 2016.

[3] (a) Beche, A. D. Density-functional exchange-energy approximation with correct asymptotic behavior. Phys. Rev. 1988, A38, 3098-3100.

(b) Beche, A. D. A new mixing of Hartree-Fock and local density - functional theories. J. Chem. Phys. 1993, 98, 1372-1377.

(c) Beche, A. D. Density-functional thermochemistry. III. The role of exact exchange. J. Chem. Phys. 1993, 98, 5648-5652.

(d) Lee, C; Yang, W.; Parr, R. G. Development of the Colle-Salvetti correlationenergy formula into a functional of the electron density. Phys. Rev. 1988, B37, 785-788.

[4] (a) Fukui, K. The Path of Chemical Reactions-the IRC Approach. Acc. Chem. Res.1981, 14, 363-368. 
(b) Ishida, K.; Morokuma, K.; Komornicki, A. The Intrinsic Reaction Coordinate. An ab initio Calculation for $\mathrm{HNC} \rightarrow \mathrm{HCN}$ and $\mathrm{H}^{-}+\mathrm{CH}_{4} \rightarrow \mathrm{CH}_{4}+\mathrm{H}^{-}$. J. Chem. Phys.1977, $66,2153-2156$.

(c) Gonzalez, C.; Schlegel, H. B. An Improved Algorithm for Reaction Path Following. J. Chem. Phys. 1989, 90, 2154-2161.

(d) Gonzalez, C.; Schlegel, H. B. Reaction Path Following in Mass-Weighted Internal Coordinates. J. Phys. Chem. 1990, 94, 5523-5527.

[5] SDD: Andrae, D.; Häußermann, U.; Dolg, M.; Stoll, H.; H. Preuß, H. EnergyAdjustedab initio Pseudopotentials for the Second and Third Row Transition Elements. Theoret. Chim. Acta 1990, 77, 123-141. 